

EVOLUTION AND ADAPTATION 
Thexizico. 


\section{EVOLUTION}

\section{AND ADAPTATION}

BY

Thomas HUNT MORGan, Ph.D.

X̌ร์ 19ork

THE MACMILLAN COMPANY

LONDON: MACMILLAN \& CO., Ltd.

1908

All rights reserved 


\section{COPYRight, IgO3,}

BY THE MACMLLAN COMPANY.

Set up and electrotypei. Published Octuber, tyo3. Reprinted January, ryos.

$$
10989
$$


TO

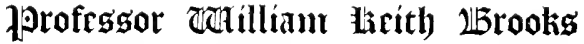

\author{
AS A TOKEN OF SINCERE ADMIRATION \\ AND RESPECT
}





\section{PREFACE}

THE adaptation of animals and plants to the conditions under which they live has always excited the interest, and also the imagination, of philosophers and scientists; for this relation between the organism and its environment is one of the most characteristic features of living things. The question at once suggests itself: How has such a relation been brought about? Is it due to something inherent in the living matter itself, or is it something that has been, as it were, superimposed upon it? An example may make my meaning clearer. No one will suppose that there is anything inherent in iron and other metals that would cause them to produce an engine if left to themselves. The particular arrangement of the pieces has been superimposed upon the metals, so that they now fulfil a purpose, or use. Have the materials of which organisms are composed been given a definite arrangement, so that they fulfil the purpose of maintaining the existence of the organism; and if so, how has this been accomplished? It is the object of the following pages to discuss this question in all its bearings, and to give, as far as possible, an idea of the present state of biological thought concerning the problem. I trust that the reader will not be disappointed if he finds in the sequel that many of the most fundamental questions in regard to adaptation are still unsettled.

In attempting to state the problem as clearly as possible, I fear that it may appear that at times I have "taken sides," 
when I should only have been justified in stating the different aspects of the question. But this will do little harm provided the issue has been sharply drawn. Indeed, it seems to me that the only scientific value, that a discussion of what the French call "les grands problèmes de la Biologie" has, is to get a clearer understanding of the relation of what is known to what is unknown or only surmised.

In some quarters speculation concerning the origin of the adaptation of living things is frowned upon, but $I$ have failed to observe that the critics themselves refrain entirely from theorizing. They shut one door only to open another, which also leads out into the dark. To deny the right to speculative thought would be to deny the right to use one of the best tools of research.

Yet it must be admitted that all speculation is not equally valuable. The advance of science in the last hundred years has shown that the kind of speculation that has real worth is that which leads the way to further research and possible discovery. Speculation that leads to this end must be recognized as legitimate. It becomes useless when it deals with problems that cannot be put to the actual test of observation or experiment. It is in this spirit that I have approached the topics discussed in the following pages.

The unsophisticated man believes that all other animals exist to minister to his welfare; and from this point of view their adaptations are thought of solely in their relation to himself. A step in advance was taken when the idea was conceived that adlaptations are for the good of the organisms themselves. It seemed a further advance when the conclusion was reached that the origin of adaptations could be accounted for, as the result of the benefit that they conferred on their possessor. This view was the outcome of the acceptation of the theory of evolution, combined with Darwin's theory of natural selection. It is the view held by most biologists at the present time; but I venture to prophesy 
that if any one will undertake to question modern zoologists and botanists concerning their relation to the Darwinian theory, he will find that, while professing in a gencral way to hold this theory, most biologists have many reservations and doubts, which they either keep to themselves or, at any rate, do not allow to interfere either with their teaching of the Darwinian doctrine or with the applications that they may make of it in their writings. The claim of the opponents of the theory that Darwinism has become a dogma contains more truth than the nominal followers of this school find pleasant to hear; but let us not, therefore, too hastily conclude that Darwin's theory is without value in relation to one side of the problem of adaptation; for, while we can profitably reject, as I believe, much of the theory of natural selection, and more especially the idea that adaptations have arisen because of their usefulness, yet the fact that living things must be adapted more or less well to their environment in order to remain in existence may, after all, account for the widespread occurrcnce of adaptation in animals and plants. It is this point of view that will be developed in the following pages.

I am fully aware of the danger in attempting to cover so wide a field as that of "Evolution and Adaptation," and I cannot hope to escape the criticism that is certain to be directed against a specialist who ventures nowadays beyond the immediate field of his own researches; yet, in my own defence, I may state that the whole point of view underlying the position here taken is the immediate outcome of my work on regeneration. One of the general questions that I have always kept before me in my study of regenerative phenomena is how such a useful acquirement as the power to replace lost parts has arisen, and whether the Darwinian hypothesis is adequate to explain the result. The conclusion that I have reached is that the theory is entirely inadequate to account for the origin of the power 
to regenerate; and it seemed to me, therefore, desirable to reëxamine the whole question of adaptation, for might it not prove true here, also, that the theory of natural selection was inapplicable? This was my starting-point. The results of my examination are given in the following pages.

I am deeply indebted to Professor G. H. Parker and to Professor E. G. Conklin for advice and friendly criticism; and in connection with the revision of the proof $I$ am under many obligations to Professor Joseph W. Warren and to Professor E. A. Andrews. Without their generous help I should scarcely have ventured into a field so full of pitfalls.

Bryn Mawr, Penn., June Io, Ig03. 


\section{CONTENTS}

\section{CHAPTER I}

The Problem of Adaptation

PAGE

Structural Adaptations

Adjustments of the Individual to Changes in the Environment . 12

Adaptations for the Good of the Species . . . . . 19

Organs of Little Use to the Individual . . . . . 22

Changes in the Organism that are of No Use to the Individual or to the Race . . . . . . . . 25

Comparison with Inorganic Phenomena . • • • 26

\section{CHAPTER II}

The Theory of Evolution.

Evidence in Favor of the Transmutation Theory . .

Evidence from Classification and from Comparative Anatomy . . . . . . . . . . 32

The Geological Evidence . . . . . . . 39

Evidence from Direct Observation and Experiment . . 43

Modern Criticism of the Theory of Evolution . . . 44

CHAPTER III

The Theory of Evolution (continued) . . . . . 58

The Evidence from Embryology . . . • • 58

The Recapitulation Theory . . . . . . 58

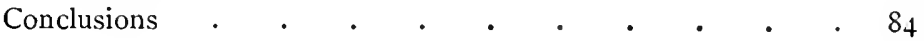

CHAPTER IV

Darwin's Theories of Artificial and of Natural Selection 91

The Principle of Selection . . . . . . . . 91

Variation and Competition in Nature . . . . . 104

The Theory of Natural Selection . . . . . . II6 


\section{CHAPTER V}

The Theory of Natural Selection (continued) • • . 129

Objections to the Theory of Natural Selection . • . . 129

Sterility between Species . • . . . . . . 147

Weismann's Germinal Selection . . . • • 。 I54

\section{CHAPTER VI}

Darwix"s Theory of Sexual Selection . • • • • $\quad$ i67

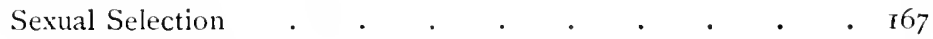

General Criticism of the Theory of Sexual Selection . . . 213

\section{CHAPTER VII}

The Inheritance of AcQuired Characters . • . 222

Lamarck's Theory . . . . . • • • 222

Darwin's Hypothesis of Pangenesis . • • • • . 233

The Neo-Lamarckian School . . . . . • 240

\section{CHAPTER VIII}

Continuous and Discontinuous Variation and Heredity • 261

Continuous Variation . . . . . . . . . 261

Heredity and Continuous Variation . . . . . . 270

Discontinuous Variation . . • • • • • • . 272

Mendel's Law . . . . . . . . . . . . 278

The Mutation Theory of De Vries . • • • • . 287

Conclusions . . • • • • • • • • 297

\section{CIIAPTER IX}

Evolution as the Result of External and Internal Factors 300

The Effect of External Influences . . . • • . 300

Responsive Changres in the Organism that adapt it to the New

Environment . . . . . . . . . . 319

Nägeli's Perfecting l'rinciple . . . . • • • • 325 
CHAPTER X

The Origin of the Different Kinds of Adaptations . 340

Form and Symmetry . . . . . . . • • 340

Mutual Adaptation of Colonial Forms . . . . . 350

Degeneration . . . . . . . . . . 352

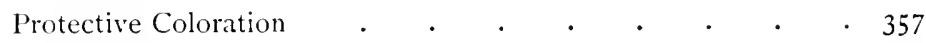

Sexual Dimorphism and Trimorphism . • • • . 360

Length of Life as an Adaptation . • • • . . 370

Organs of Extreme Perfection . . • • • • • 371

Secondary Sexual Organs as Adaptations . • • • 372

Individual Adjustments as Adaptations . . • • • 375

Color Changes as Individual Adaptations . . . . • • 375

Increase of Organs through Use and Decrease through Disuse • 376

Reactions of the Organisn to Poisons, etc. • • • • 377

Regeneration . • • • • • • • • • 379

CHAPTER XI

Tropismis and Instincts as Adaptations . • • • $\quad 3^{8 z}$

CHAPTER XII

Sex as an Adaptation . . . . . . . . . 414

The Different Kinds of Sexual Individuals . • • • 4 It

The Determination of Sex . . . • • • • 422

Sex as a Phenomenon of Adaptation . • • • • 439

CHAPTER XIII

Summary and General Conclusions • • • • • • 4452

$\operatorname{INDEX} \cdot \quad \cdot \quad \cdot \quad \cdot \quad \cdot \quad \cdot \quad \cdot \quad \cdot \quad 465$ 



\title{
EVOLUTION AND ADAPTATION
}

\author{
CHAPTER I \\ THE PROBLEM OF ADAPTATION
}

Between an organism and its environment there takes place a constant interchange of energy and of material. This is, in general, also true for all bodies whether living or lifeless ; but in the living organism this relation is a peculiar one; first, because the plant or the animal is so constructed that it is suited to a particular set of physical conditions, and, second, because it may so respond to a change in the outer world that it further adjusts itself to changing conditions, $i e$. the response may be of such a kind that it better insures the existence of the individual, or of the race. The two ideas contained in the foregoing statement cover, in a general way, what we mean by the adaptation of living things. The following examples will serve to illustrate some of the very diverse phenomena that are generally included under this head.

\section{Structural Adaptations}

The most striking cases of adaptations are those in which a special, in the sense of an inusual, relation exists between the individual and its surroundings. For example, the foreleg of the mole is admirably suited for digging underground. A similar modification is found in an entirely different group of the animal kingdom, namely, in the mole-cricket, in which the first legs are also well suited for digging. By their use the mole-cricket makes a burrow near the surface of the ground, 
similar to, but of course much smaller than, that made by the mole. In both of these cases the adaptation is the more obvious, because, while the leg of the mole is formed on the same general plan as that of other vertebrates, and the leg of the mole-cricket has the same funclamental structure as that of other insects, yet in both cases the details of structure and the general proportions have been so altered, that the leg is fitted for entirely different purposes from that to which the ligs of other vertebrates and of other insects are put. The wing of the bat is another excellent case of a special adaptation. It is a modified fore-limb having a strong membrane stretched between the fingers, which are greatly elongated. Here we find a structure, which in other mammals is used as an organ for supporting the body, and for progression on the ground, changed into one for flying in the air.

The tails of mammals show a number of different adaptations. The tail is prehensile in some of the monkeys; and not only can the monkey direct its tail toward a branch in order to grasp it, but the tail can be wrapped around the branch and hold on so firmly that the monkey can swing freely, hanging by its tail alone. The animal has thus a sort of fifth hand, one as it were in the middle line of the body, which can be used as a hold-fast, while the fingered hands are put to other uses. In the squirrels the bushy tail serves as a protection dluring the winter for those parts of the body not so thickly covered by hair. The tail of the horse is used to brush away the flies that settle on the hind parts of the body. In other mammals, the dog, the cat, and the rat, for example, the tail is of less obvious use, although the suggestion has been made that it may serve as a sort of rudder when the animal is running rapidly. In several other cases, as in the rabbit and in the higher apes, the tail is very short, and is of no apparent use; and in man it has completely disappeared.

A peculiar case of adiutation is the so-called basket on the third pair of legs of the worker honey-bee. A depression 
of the outer surface of the tibia is arched over by stiff hairs. The pollen collected from the stamens of flowers is stowed away in this receptacle by means of the other pairs of legs. The structure is unique, and is not found in any other insects except the bees. It is, moreover, present only in the worker bees, and is absent in the queen and the males.

The preceding cases, in which the adapted parts are used for the ordinary purposes of life of the individual, are not essentially different from the cases in which the organ is used to protect the animal from its enemies. The bad taste of certain insects is supposed to protect them from being eaten by birds. Cases like this of passive protection grade off in turn into those in which, by some reflex or voluntary act, the animal protects itself. The bad-smelling horns of the caterpillar of the black swallow-tailed butterfly (Papilio polyrenes) are thrust out when the animal is touched, and it is believed that they serve to protect the caterpillar from attack. The fotid secretion of the glands of the skunk is believed to serve as a protection to the animal, although the presence of the nauseous odor may lead finally to the extermination of the skunk by man. The sting of bees and of wasps serves to protect the individual from attack. The sting was originally an ovipositor, and used in laying the eggs. It has, secondarily, been changed into an organ of offence.

The special instincts and reflex acts furnish a striking group of adaptations. The building of the spider's web is one of the most remarkable cases of this kind. The construction of the web cannot be the result of imitation, since, in many instances, the young are born in the spring of the year following the death of the parents. Each species of spider has its own type of web, and each web has as characteristic a form as has the spider itself. It is also important to find that a certain type of web may be characteristic of an entire family of spiders. Since, in many cases, the web 
is the means of securing the insects used for food, it fulfils a purpose necessary for the welfare of the spider.

The making of the nests by birds appears to be also in large part an instinctive act; although some writers are inclined to think that memory of the nest in which the young birds lived plays a part in their actions, and imitation of the old birds at the time of nest-building may, perhaps, also enter into the result. It has been stated that the first nest built by young birds is less perfect than that built by older birds, but this may be due to the bird's learning something themselves in building their nests, i.c. to the perfecting of the instinet in the inclividual that makes use of it. In any case much remains that must be purely instinctive. The construction of the comb by bees appears to be largely, perhaps entirely, an instinctive act. That this is the case was shown by isolating young workers as soon as they emerged from the cell, and before they could have had any experience in seeing comb built. When given some wax they set to work to make a comb, and made the characteristic six-sided structures like those made by the bees in a hive. The formation of so remarkable a structure as the comb is worthy of admiration, for, with the greatest economy of material, a most perfect storeroom for the preservation of the honey is secured. This adaptation appears almost in the nature of foresight, for the store of honey is used not only to feed the young, but may be drawn on by the bees themselves in time of need. It is true that a comparison with other kinds of bees makes it probable that the comb was first made for the eggs and larva, and only later became used as a storehouse, but so far as its form is concerned there is the same economy of constructive materials in either case.

The behavior of young bircls, more especially those that take care of themselves from the moment they leave the egg, furnishes a number of cases of instincts that are protective. If, for example, a flock of young pheasants is suddenly dis- 
turbed, the birds at once squat down on the ground, and remain perfectly quiet until the danger is past. Their resemblance to the ground is so perfect that they are almost invisible so long as they remain quiet. If, instead of remaining still, they were to attempt to run away when disturbed, they would be much more easily seen.

Certain solitary wasps (Ammoplitla) have the habit of stinging caterpillars and spiders, and dragging them to their nests, where they are stored away for the future use of the young that hatch from the eggs laid by the wasp on the bocly of the prey. As a result of the sting which the wasp administers to the caterpillar, the latter is paralyzed, and cannot escape from the hole in which it is stored, where it serves as food for the young wasp that emerges from the egg. It was originally claimed by Forel that the wasp stings the caterpillar in such a way that the central nervous system is always pierced, and many subsequent naturalists have marvelled at the perfection of such a wonderful instinct. But the recent results of the Peckhams have made it clear that the act of the wasp is not carried out with the precision previously supposed, although it is true that the wasp pierces the caterpillar on the lower surface where the ventral chain of ganglia lies. The habit of this wasp is not very dissimilar from that shown by many other kinds of wasps that sting their captive in order to quiet it. We need not imagine in this case that the act carries with it the consciousncss that the caterpillar, quieted in this way, will be unable to escape before the young wasps have hatched.

The resemblance in color of many animals to their natural backgrounds has in recent years excited the interest and imagination of many naturalists. The name of protective coloration has been given to this group of phenomena. The following cases which have less the appearance of purely imaginative writing may serve by way of illustration. A striking example is that of the ptarmigan which has a pure 
white coat in winter, and a brown coat in summer. The white winter plumage renders the animal less conspicuous against the background of snow, while in summer the plumage is said to closely resemble the lichen-covered ground on which the bird rests. The snowy owl is a northern bird, whose color is supposed to make it less conspicuous, and may serve either as a protection against enemies, or may allow the owl to approach its prey unseen. It should not pass unnoticed, however, that there are white birds in other parts of the world, where their white color cannot be of any use to them as a protection. The white cockatoos, for example, are tropical birds, living amongst green foliage, where their color must make them conspicuous, rather than the reverse.

The polar bear is the only member of the family that is white, and while this can searcely be said to protect it from enemies, because it is improbable that it has anything to fear from the other animals of the ice-fields, yet it may be claimed that the color is an adaptation to allow the animal to approach unseen its prey.

In the desert many animals are sand-colored, as seen for instance in the tawny color of the lion, the giraffe, the antelopes, and of many birds that live on or near the ground.

It has been pointed out that in the tropies and temperate zones there are many greenish and yellowish birds whose colors harmonize with the green and yellow of the trees amongst which they live; but on the other hand we must not forget that in all elimes there are numbers of birds brilliantly colored, and many of these do not appear to be protected in any special way. The tanagers, humming-birds, parrots, Chinese pheasants, birds of paradise, etc., are extremely conspicuous, and so far as we can see they must be much exposed on account of the color of their plumage. Whether, therefore, we are justified in picking out certain cases as examples of adaptation, because of an agreement in 


\section{The Problem of Adaptation}

color between the organism and its surroundings, and in neglecting all others, is, as has been already said, a point to be further examined.

Not only among mammals and birds have many cases of protective coloration been described by writers dealing with this subject, but in nearly every group of the animal kingdom similar cases have been recognized. The green and brown color of lizards may protect them, the green color of many frogs is supposed to conceal them as they sit amongst the plants on the edge of a stream or pond. The gray-brown color of the toad has been described as a resemblance to the dry ground, while the brilliant green of several tree-frogs conceals them very effectively amongst the leaves. Many fishes are brilliantly colored, and it has even been suggested that those living amongst corals and sea-anemonies have acquired their colors as a protection, but Darwin states that they appeared to him very conspicuous even in their highly colored environment.

Amongst insects innumerable cases of adaptive coloration have been described. In fact this is the favorite group for illustrating the marvels of protective coloration. A few examples will here serve our purpose. The oft-cited case of the butterfly Kallima is, apparently, a striking instance of protective resemblance. When at rest the wings are held together over the back, as in nearly all butterflies, so that only the under surface is exposed. This surface has an unquestionably close resemblance to a brown leaf. It is said on no less authority than that of Wallace that when this butterfly alights on a bush it is almost impossible to distinguish between it and a dead leaf. The special point in the resemblance to which attention is most often called is the distinct line running obliquely across the wings which looks like the midrib of a leaf. Whether the need of such a close resemblance to a leaf is requisite for the life of this butterfly, we do not know, of course, and so long as we do not have 
this information there is danger that the case may prove too much, for, if it should turn out that this remarkable case is accidental the view in regard to the resemblance may be endangered.

Amongst caterpillars there are many cases of remarkable resemblances in color between the animal and its surroundings. The green color of many of those forms that remain on the leaves of the food-plant during the day will give, even to the most casual observer, the impression that the color is for the purpose of concealment; and that it does serve to conceal the animal there can be no doubt. But even from the point of view of those who maintain that this color has been acquired because of its protective value it must be admitted that the color is insufficient, because some of these same green caterpillars are marvellously armed with an array of spines which are also supposed to be a protection against enemies. Equally well protected are the brown and mottled geometrid caterpillars. These have, moreover, the striking and unusual habit of fixing themselves by the posterior pairs of false legs, and standing still and rigid in an oblique position on the twigs to which they are affixed. So close is their resemblance to a short twig, that even when their exact position is known it is very difficult to distinguish them.

Grasshoppers that alight on the ground are, in many cases, so similar to the surface of the ground that unless their exact location is known they easily escape attention, while the green color of the katydid, a member of the same group of orthoptera, protects it from view in the green foliage of the trees where it lives. The veinlike wings certainly suggest a resemblance to a leaf, but whether there is any necessity for so close an imitation may be questioned.

There can be little doubt in some of these cases that the color of the animal may be a protection to it, but as has been hinted already, it is another question whether it acquired these colors because of their usefulness. Never- 
theless, if the color is useful to its possessor, it is an adaptation in our sense of the word, without regard to the way in which it has been acquired. Even, for instance, if the resemblance were purely the outcome of chance in the sense that the color appeared without relation to the surroundings, it would still be an adaptation if it were of use to the animal under the ordinary conditions of life.

In the lower groups numerous cases in which animals resemble their surroundings could be given. Such cases are known in crustacea, worms, mollusks, hydroids, etc., and the possible value of these resemblances may be admitted in many instances.

It is rather curious that so few cases of adaptive coloration have been described for plants. No one supposes that the slate color of the lichen is connected with the color of the rocks on which it grows, in the sense that the resemblance is of any use to the lichen. Nor does the color of the marine red algæ serve in any way to protect the plants so far as is known. The green color of nearly all the higher plants is obviously connected with the substance, chlorophyl, that is essential for the processes of assimilation, and has no relation to external objects. But when we come to the colors of flowers we meet with curious cases of adaptation, at least according to the generally accepted point of view. For it is believed by many naturalists that the color of the corolla of flowering plants is connected with the visits of insects to the flowers, and these visits are in many cases essential for the cross-fertilization of the flowers. This adaptation is one useful to the species, rather than the individual, and belongs to another category.

The leaf of the Venus's fly-trap, which suddenly closes together from the sides when a fly or other light body comes to rest on it, is certainly a remarkable adaptation. A copious secretion of a digestive fluid is poured out on the surface of the leaf, and the products of digestion are absorbed. 
There can be no question that this contrivance is of some use to the plant. In other insectivorous plants, the pitcher plants, the leaves are transformed into pitchers. In Nepenthes a digestive fluid is secreted from the walls. A line of glands secreting a sweet fluid serves to attract insects to the top of the pitcher, whence they may wander or fall into the fluid inside, and there being drowned, they are digested. A lidlike cover projecting over the opening of the pitcher is supposed to be of use to keep out the rain.

In Utricularia, a submerged water-plant, the tips of the leaves are changed into small bladders, each having a small entrance closed by an elastic valve opening inwards. Small snails and crustaceans can pass into this opening, to which they are guided by small outgrowths; but once in the cup they cannot get out again, and, in fact, small animals are generally found in the bladders where they die and their substance is absorbed by forked hairs projecting into the interior of the bladder.

The cactus is a plant that is well suited to a dry climate. Its leaves have completely disappeared, and the stem has become swollen into a water-1eservoir. "It has been estimated that the amount of water evaporated by a melon cactus is reduced to one six-hundredth of that given off by any equally heavy climbing-plant."

Sachs gives the following account of the fertilization process in Aristolochia Clematitis, which he refers to as a conspicuous and peculiar adaptation. In Figure I A a group of flowers is shown, and in Figure $\mathbf{I}$ B and $C$ a single flower is split open to show the interior. In B a small fly has entered, and has brought in upon its back some pollen that has stuck to it in another flower. The fly has entered through the long neck which is beset with hairs which are turned inwards so that the fly can enter but cannot get out. In roaming about, the pollen that is sticking to its back will be rubbed against the stigmatic surface. 
"As soon as this has taken place the anthers, which have been closed hitherto, dehisc and become freely accessible," as a result in the change in the stigma and of the collapse of the hairs at the base of the enlargement which has widened. The fly can now crawl under the anthers, and,

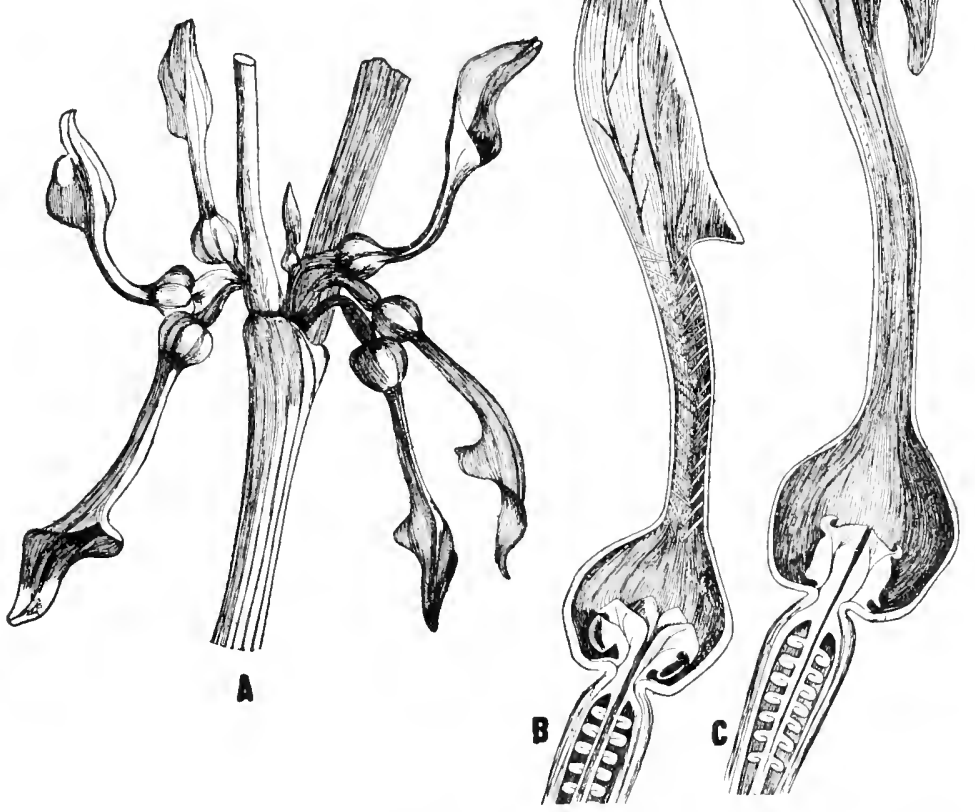

FIG. 1. - The fertilization of Aristolochia Clematitis. A, portion of stem with flowers in axil of leaf in different stages. B, longitudinal sections of two flowers, before and after fertilization. (After Sachs.)

if it does so, new pollen may stick to its back. At this time the hairs in the throat dry up, and the fly can leave its prison house, Figure I C. If the fly now enters another flower this is fertilized by repeating the process. The unfertilized flowers stand erect with widely open mouths. As soon as they have been fertilized they bend down, as seen in 
Figure I A, and at the same time the terminal flap bends over the open mouth of the throat, "stopping the entrance to the flies, which have now nothing more to do here."

\section{Adjustments of the Indifidual to Chasges in the ENVIRONIENT}

The most familiar cases of adjustments of the individual to the environment are those that we recognize in our own bodies. After violent exercise we breathe more rapidly, and take deeper inspirations. Since during exercise our blood loses more oxygen and takes in more carbon dioxide from the muscles, it is clear that one result of more rapid breathing is to get more oxygen into the blood and more carbon dioxide out of it. The process of sweating, that also follows exercise, may be also looked upon as an adaptive process, since by evaporation the skin is kept cooler, and, in consequence, the blood, which at this time flows in larger quantities to the skin, is cooled also.

More permanent adaptive changes than these also take place as the result of prolonged use of certain parts. If the muscles work against powerful resistance, they become larger after several days or weeks, and are capable of doing more work than at first. Conversely, when any group of muscles is not used, it becomes smaller than the normal and capable of doing less work. It would be a nice point to decide whether this latter change is also an adaptation. If so it is one in a somewhat different sense from that usually employed. The result is of no direct advantage to the animal, except possibly in saring a certain amount of food, but since the same change will take place when an abundance of food is consumed, the result is, under these conditions, of no use.

The thickening of the skin on those parts of the body where continued pressure is brought to bear on it is a change in a useful direction. The thickening on the soles of the feet and 
on the palms of the hands is a case in point. Not only is the skin thicker at birth in these parts, but it becomes thicker through use. In other parts of the body also, the skin hardens and becomes thicker if pressure is brought to bear on it. We may regard this as a general property of the skin, which is present even in those parts where, under ordinary circumstances, it can rarely or never be brought into use.

Even as complicated and as much used an organ as the eye can become adaptively improved. It is said that the lateral region of the field of vision can be trained to perceive more accurately; and every one who has used a microscope is familiar with the fact that if one eye is habitually used it becomes capable of seeing more distinctly and better than the other eye. This seems to be due, in part at least, to the greater contraction of the iris.

Another phenomenon, which, I think, must be looked upon as an adaptation, is the immunity to certain poisons that can be gradually brought about by slowly increasing the amount introduced into the body. Nicotine is a most virulent poison, and yet by slowly increasing the dose an animal can be brought into a condition in which an amount of nicotine, fatal to an ordinary individual, can be administered without any ill effects at all resulting.

The same phenomenon has been observed in the case of other poisons, not only in case of other alkaloids, such as morphine and cocaine, but also in the case of caffein, alcohol, and even arsenic. There is a curious phenomenon in regard to arsenic, which appears to be well established, viz., that a person who has gradually increased the dose to an amount great enough to kill ten ordinary men, will die if he suddenly ceases altogether to take arsenic. He can, however, be gradually brought back to a condition in which arsenic is not necessary for his existence, if the dose is gradually decreased. It is a curious case of adaptation that we meet with here, since the man becomes so thoroughly adjusted to a poison 
that if he is suddenly brought back to the normal condition of the race he will die.

Immunity to the poison of venomous snakes can also be acquired by slowly increasing the amount given to an animal. It is possible to make a person so immune to the poison of venomous snakes that he would become, in a sense, adapted to live amongst them without danger to himself. It is to be noted, moreover, that this result could be reached only by quite artificial means, for, under natural conditions it is inconceivable that the nicely graded series of doses of increasing strength necessary to bring about the immunity could ever be acquired. Hence we find here a case of response in an adap. tive direction that could not have been the outcome of experience in the past. It is important to emphasize this capacity of organisms to adapt themselves to certain conditions entirely new to them.

These cases lead at once to cases of immunity to certain bacterial diseases. An animal may become immune to a particular disease in several ways. First, by having the disease itself, which renders it immune for a longer or a shorter period afterwards; or, second, by having a mild form of the disease as in the case of smallpox, where inmunity is brought about by vaccination, i.c. by giving the individual a mild form of smallpox; or, third, by introducing into the blood an antidote, in the form, for example, of antitoxin, which has been made by another animal itself immune to the disease. The first two classes of immunity may be looked upon as adaptations which aie of the highest importance to the organism; the last case can scarcely be looked upon as an adaptive process, since the injurious effect of the poison may as well be neutralized outside of the body by mixing it with the antitoxin. We may suppose, then, that in the body a similar process goes on, so that the animal itself takes no active part in the result.

When we consider that there are a number of bacterial 
diseases, in each of which a different poison is made by the bacteria, we cannot but ask ourselves if the animal really makes a counter-poison for each disease, or whether a single substance may not be manufactured that counteracts all alike? That the latter is not the case is shown by the fact that an animal made immune to one disease is not immune to others. When we recall that the animal has also the capacity to react in one way or another to a large number of organic and inorganic poisons, to which it or its ancestors can have had little or no previous experience, we may well marvel at this wonderful regulative power.

The healing of wounds, which takes place in all animals, forms another class of adaptive processes. The immense usefulness of this power is obvious when it is remembered how exposed most animals are to injuries. By repairing the injury the animal can better carry on its normal functions. Moreover, the presence of the wound would give injurious bacteria a ready means of entering the body. In fact, an intact skin is one of the best preventives to the entrance of bacteria.

Not only have most organisms the power of repairing injuries, but many animals have also the closely related power of regenerating new parts if the old ones are lost. If a crab loses its leg, a new one is regenerated. If a freshwater worm (Lumbriculus) is cut into pieces, each piece makes a new head at its anterior end and a new tail at the posterior end. In this way as many new worms are produced as there are pieces. And while in a strict sense it cannot be claimed that this power of regeneration is of any use to the original worm, since the original worm, as such, no longer exists, yet since it has not died but has simply changed over into several new worms, the process is of use inasmuch as by this means the pieces can remain in existence.

We need not discuss here the relative importance to different animals of this power of regeneration, but it may be stated, 
that, while in some cases it may be necessary to replace the lost part if the animal is to remain in existence, as when a new head is formed on an earthworm after the old one was cut off, in other cases the replacement of the lost part appears to be of minor importance, as in the case of the leg of the crab. While we are not, for the moment, concerned with the relative importance of the different adaptations, this question is one of much importance in other connections and will be considered later.

The protective coloration of some animals, which is the direct result of a change in color of the animal in response to the surroundings, furnishes us with some most striking cases of adaptive coloration. A change of this sort has been recorded in a number of fishes, more especially in the flounders. The individuals found living on a dark background are darker than those living on a lighter background; and when the color of the background is changed it has been observed that the color of the fish also changes in the same direction. I have observed a change of this sort from clark to light, or from light to clark, in the common minnow (Fundulus) in accordance with a change of its background, and the same sort of change appears to take place in many other fishes.

The change from green to brown and from brown to green in certain tree frogs and in the lizard (Anolis), which is popularly supposed to take place according to whether the background is green or brown, is not after all, it appears, connected with the color of the background, but depends on certain other responses of the animals that have not yet been satisfactorily made out. If it be claimed that in summer the animal would generally be warm, and therefore, often green, and that this color would protect it at this time of year when the surroundings are green, and in winter brown, when this color is the prevailing one in temperate regions, then it might appear that the change is of use to 


\section{The Problem of Adaptation}

the animal; but if it is true that the same change takes place in some of the lizards that live in the tropics, where the prevailing color is always green, it would appear that the result may have no direct relation with the surroundings. It has been shown in a number of well-authenticated cases that the pupæe of certain butterflies vary in color within certain limits in response to the color of the background. When the caterpillar fixes itself to some surface, and there throws off the outer skin, and acquires a new one, the color of the latter is influenced by the background. The result is a better protection to the pupa. The change is not brought about through the ocelli or eyes, but through the general surface of the skin, for the same change takes place when the eyes have been previously covered with a dark pigment.

The growth of plants toward the light may be looked upon as an adaptive process, since only in the light can they find the conditions necessary for their life. The extraordinary elongation of shoots and young plants when grown in the dark may also be considered an adaptation for finding the light, since in this way a plant, deeply embedded in the ground, may ultimately reach the surface. Thus while the actual process of elongation in the dark is not in itself of any use, yet under the ordinary conditions of its life, this response may be of great benefit to the plant.

The closing together of the leaves of some plants has been supposed to protect them from too rapid radiation of heat, and incidentally this purpose may be fulfilled; but since some tropical plants also close their leaves during the night, it can hardly be maintained that the closing has been acquired for this purpose. It has been suggested that the opening of certain flowers under certain conditions of light is connected with the visits of insects that bring about crossfertilization.

The preceding examples will suffice to give a general idea of what is meant by adaptation in organisms. That 
the term includes a large number of phenomena of very different kinds is apparent. When we have examined these phenomena further we shall find, I think, that it will be necessary to put some of them into different categories and treat them differently. It is probably incorrect to suppose that all processes useful to the organism have been acquired in the same way, nevertheless, for the present the term adaptation is sufficiently general, even if vague, to cover these different groups of cases.

It may be asked, in what respects are these structures and processes of adaptation different from the ordinary structures and changes that go on in the organism? Why is the leg of the mole more of an adaptation than that of a dog? The one is of as much use as the other to its possessor. What reason can we give for citing the poison of the snake, and not mentioning in the same connection the other glands of the body? In fact, the poison gland of the snake is supposed to be a modified superior labial gland. WVhy, in short, are not the processes of digestion, excretion, secretion, the beating of the heart, the ordinary reflex acts of the nervous system, and the action of the sense organs, as truly adaptations as the special cases that have been selected for illustration. The answer is simply that we are more impressed by those cases of adaptation that are more unusual, as when an animal departs in the use of certain structures from the rest of the group to which it belongs. For example, if all mammals lived underground, ourselves included, and the fore-legs or arms were used for burrowing, we should not think this unusual; but if we found an animal using all four legs to support the body and for purposes of progression, we should, most likely, think this was an excellent illustration of adaptation.

In other instances the condition is somewhat different. The color of certain animals may unquestionably be of use to them in concealing them from their enemies. In other 
cases the color may not serve this purpose, or any purpose at all. Thus while in the former case we speak of the color as an adaptation to the surroundings, in the latter we do not think of it as having any connection at all with the environment. Even in the same animal the color of different parts of the body may appear under this twofold relation. For example, the green color of the skin of the frog renders it less conspicuous amongst the green plants on the edge of the stream, but the brilliant orange and black pigment in the body-cavity cannot be regarded as of any use to the animal.

\section{Adaptations for the Good of the Species}

Aside from the class of adaptations that are for the good of the individual, there is another class connected solely with the preservation of the race. The organs for reproduction are the most important examples of this kind. These organs are of no use to the individual for maintaining its own existence, and, in fact, their presence may even be deleterious to the animal. The instincts connected with the use of these organs may lead inevitably to the death of the individual, as in the case of the California salmon, which, on entering fresh water in order to deposit its eggs, dies after performing this act.

The presence of the organs of reproduction in the individual is obviously comnected with the propagation of other individuals. Indeed in many organisms the life of the individual appears to have for its purpose the continuation of the race. In a large number of animals the individual dies after it has deposited its eggs. The most striking case is that of the May-flies, whose life, as mature individuals, may last for only a few hours. The eggs are set free by the bursting of the abdomen, and the insect dies. The male bee also dies after union with the queen. In some annelids, the body is also said to burst when the eggs are set free; 
and in other forms those parts of the body containing the eggs break off, and, after setting free the eggs, die. These are extreme cases of what is seen in many animals, namely the replacement of the old individuals by a new generation; and while in general there is only a loose connection between the death of the individual and the consummation of its reproductive power, yet the two run a course so nearly parallel that several writers have attempted to explain this connection as one of racial adaptation.

It has aiso been pointed out that in those higher animals that take care of their young after birth, the life of the individual does not end with the period of birth of the young, but extends at least throughout the time necessary to care for the young. It has even been suggested that this lengthening of the life period has been acquired on account of its use to the species. When, however, as in the case of the vertebrates, the young are born at intervals either in great numbers at a birth, as in fishes and amphibia, or in lots of twos, threes, or fours, as in many birds and mammals, or even only one at a time, as in a few birds and in man, it will be evident that the relation cannot be so simple a has been supposed. It cannot be assumed in these forms that the end of the life of the individual is in any way connected with the ripening of the last eggs, for, on the contrary, hundreds, or even many thousands, of potential eggs may be present in the ovaries when the animal is overtaken by old age, and its power of reproduction lost.

In regard to several of the lower animals, we find, in a number of cases where there are accurate data, that the individual goes on year after year producing young. Whether they ever grow old, in the sense of losing their power of reproduction, has not been definitely determined, but there is, so far as I know, no evidence to show that such a process takes place, and these animals appear to have the power of reproducing themselves indefinitely. 
The phenomenon of old age (apart from its possible connection with the cessation of the power of reproduction), which leads to the death of the individual, has been looked upon by a few writers as an adaptation of the individual for the good of the species. It has been pointed out by these writers that the longer an individual lives, the more likely it is to become damaged, and if along with this its powers of reproduction diminish, as compared with younger individuals, then it stands in the way and takes food that might be used by other, younger individuals, that are better able to carry on the propagation of the race. It is assumed, therefore, that the life of the individual has been shortened for the benefit of the race. Whether such a thing is probable is a question that will also be discussed later. We are chiefly concerned here only in recording the different groups of phenomena that have been regarded by biologists as adaptations.

The so-called secondary sexual characters such as the brighter colors of the males, ornaments of different kinds, crests, color-pattern, tail feathers, etc., organs of offence and of defence used in fighting members of the same species, present a rather unique group of adaptations. These characters are supposed to be of use to the individual in conquering its rivals, or in attracting the females. They may be considered as useful to the individual in allowing it to propagate at the expense of its rivals, but whether the race is thereby benefited is a question that will be carefully considered later.

The colors of flowers, that is supposed to attract insects, have been already mentioned. The sweet fluid, or nectar, secreted by many flowers is sought by insects, which on entering the flowers bring about cross-fertilization. Thus while the nectar seems to be of no immediate service to the plant itself, it is useful to the species in bringing about the fertilization of the flowers. The odors of flowers also serve to attract insects, and their presence is one of the means by which insects find the flowers. This also is of advantage to the race. 


\section{Organs of Little Use to the Individual}

In every organism there are parts of the body whose presence cannot be of vital importance to the individual. We may leave out of consideration the reproductive organs, since their presence, as has just been stated, is connected with the continuation of the race. The rudimentary organs, so-called, furnish many examples of structures whose presence may be of little or of no use to the individual; in fact, as in the case of the appendix in man, the organs may be a source of great danger to the individual. In this respect the organism is a structure not perfectly adapted to its conditions of life, since it contains within itself parts that are of little or of no use, which may even lead to its destruction, and may often expose it to unnecessary danger. Nevertheless such parts are surprisingly infrequent, and their presence is usually accounted for on the supposition that in the past these organs have been of use, and have only secondarily come to play an insignificant part in the functions of the organism. Another example of the same thing is found in the rudimentary eyes of animals living in the dark, such as the mole and several cave animals, fishes, amphibia, and insects.

There are still other organs, which cannot be looked upon as rudimentary, yet whose presence can scarcely be considered as essential to the life of the individual. It is with this class that we are here chicfly concerned. For instance, the electric organs in some of the rays and fish can hardly protect the animal from enemies, even when as highly developed as in the torpedo: and we do not know of any other essential service that they can perform. Whether the same may be also said of the phosphorescent organs of many animals is perhaps open in some cases to doubt, but there can be little question that the light produced by most of the small marine organisms, such as noctiluca, jellyfish, ctenophores, copepods, pyrosoma, etc., cannot be of use to these 
animals in protecting them from attack. In the case of certain bacteria it seems quite evident that the production of light can be of no use as such to them. The production of light may be only a sort of by-product of changes going on in the organism, and have no relation to outside conditions. In certain cases, as in the glowworm, it has been supposed that the display may serve to bring the sexes together; but since the phosphorescent organs are also present in the larval stages of the glowworm, and since even the egg itself is said to be phosphorescent, it is improbable, in these stages at least, that the presence of the light is of service to the organism.

It has been pointed out that the colors of certain animals may serve to conceal them and may be regarded as an adaptation; but it is also true that in many cases the color of the whole animal or the color of special parts can be of little if any direct use. While it is clifficult to show that the wonderful patterns and magnificent coloration of many of the larger animals are not of service to the animal, however sceptical we may be on the subject, yet in the case of many microscopical forms that are equally brilliantly colored there can be little doubt that the coloration can be of no special service to them. If it be admitted that in these small forms the color and the color patterns are not protective, we should at least be on our guard in ascribing off-hand to larger forms a protective value in their coloration, unless there is actual proof that it serves some purpose.

We also see in other cases that the presence of color need not be connected with any use that it bears as such to the animal. For instance, the beautiful colors on the inside of the shells of many marine snails and of bivalve mollusks, can be of no use to the animal that makes the shell, because as long as the animal is alive this color cannot be seen from the outside. This being the case let us not jump too readily to the conclusion that when other shells are colored on the outer surface that this must be of use to the mollusk. 
In regard to the colors of plants, there are many cases of brilliant coloration, which so far as we can see can be of no service to the organism. In such forms as the lichens and the toadstools, many of which are brilliantly colored, it is very doubtful if the color, as such, is of any use to the plant. The splendid coloring of the leaves in the autumn is certainly of no service to the trees.

It should not pass unnoticed in this connection that the stems and the trunks of shrubs and of trees and also many kinds of fruits and nuts are sometimes highly colored. It is true that some of the latter have been supposed to owe their color to its usefulness in attracting birds and other animals which, feeding on the fruit, swallow the seeds, and these, passing through the digestive tract and falling to the ground, may germinate. The dissemination of the seeds of such plants is supposed to be brought about in this way; and since they may be widely disseminated it may be supposed that it is an advantage to the plant to have attracted the attention of the fruit-eating birds. On the other hand one of the most brilliantly colored seeds, the acorn, is too large to pass through the cligestive tracts of birds, and is, in fact, ground to pieces in the gizzard, and in the case of several mammals that feed on the acorns, the acorn is crushed by the teeth. It would seem, therefore, that its coloration is injurious to it rather than the reverse, as it leads to its destruction. It has been suggested by Darwin that since the acorns are for a time stored up in the crop of the bird, the passenger pigeon for example, and since the birds may be caught by hawks and killed, the seeds in the crop thus become scattered. Consequently it may be, after all, of use to the oak to produce colored acorns that attract the attention of these pigeons. This suggestion seems too farfetched to consider seriously. In the case of the horsechestnut the rich brown color is equally conspicuous, but the nut is too large to be swallowed by any of the ordinary 
seed-feeding birds or mammals. Shall we try to account for its color on the grounds of the poisonous character of the seed? Has it been acquired as a warning to those animals that have eaten it once, and been made sick or have died in consequence? I confess to a personal repugnance to imaginative explanations of this sort, that have no facts of experience to support them.

Changes in the Organism that are of No Use to the INDIVIDUAL OR TO THE RACE

As an example of a change in the organism that is of no use to it may be cited the case of the turning white of the hair in old age in man and in several other mammals. The absorption of bone at the angle of the chin in man, is another case of a change of no immediate use to the individual. We also find in many other changes that accompany old age, processes going on that are of no use to the organism, and which may, in the end, be the cause of its death. Such changes, for instance, as the loss of the vigor of the muscles, and of the nervous system, the weakening of the heart, and partial failure of many of the organs to carry out their functions. These changes lead sooner or later to the death of the animal, in consequence of the breaking down of some one essential organ, or to disease getting an easier foothold in the body. We have already discussed the possible relation of death as an adaptation, but the changes just mentioned take place independently of their relation to the death of the organism as a whole, and show that some of the nor. mal organic processes are not for the good of the individual or of the race. In fact, the perversions of some of the most deeply seated instincts of the species, as in infanticide, while the outcome of definite processes in the organism, are of obvious disadvantage to the individual, and the perversion of so deeply seated a process as the maternal instinct, leading 
to the destruction of the young, is manifestly disadvantageous to the race. As soon, however, as we enter the field of socalled abnormal developments, the adaptive relation of the organism to its environment is very obscure; and yet, as in the case of adaptation to poisons, we see that we cannot draw any sharp line between what we call normal and what we call abnormal development.

\section{Comparison with Inorganic Phenomena}

The preceding examples and discussion give some idea of what is meant by adaptation in living things. In what respects, it may be asked, do these adaptations differ from inorganic phenomena? The first group of inorganic bodies that challenges comparison are machines. These are so constructed that they may be said to accomplish a definite purpose, and the question arises whether this purpose can be profitably compared with the purposefulness of the structure and response of organisms. That the two cannot be profitably compared is seen at once, when we recall the fact that the activity of the machine is of no use to it, in the sense of preserving its integrity. The object of the machine is, in fact, to perform some useful purpose for the organism that built it, namely, for man. Furthermore, the activity of the machine only serves to wear it out, and, therefore, its actions do not assist in preserving its integrity as do some, at least, of the activities of an animal. It is true, of course, that in a mechanical sense every action of the organism leads also to a breaking down of its structure in the same way that a machine is also worn out by use; but the organism possesses another property that is absent in the machine, namely, the power of repairing the loss that it sustains.

One of the most characteristic features of the organism is its power of self-adjustment, or of regulation, by which it adapts itself to changes in the environment in such a way 
that its integrity is maintained. Most machines have no such regulative power, although, in a sense, the fly-wheel of an engine regulates the speed, and a water-bath, with a thermostat, regulates itself to a fixed temperature; but even this comparison lacks one of the essential features of the regulation seen in organisms, namely, in that the regulation does not protect the machine from injury. It may be claimed, however, that the safety valve of an engine does fulfil this purpose, since it may prevent the engine from exploding. Here, in fact, we do find better grounds for comparison, but, when we take into account the relation of the regulations in the organism to all the other properties of the organism, we see that this comparison is not very significant. The most essential difference between a machine and an organism is the power of reproduction possessed by the latter, which is absent in all machines. Here, however, we meet with a somewhat paradoxical relation, since the reproductive power of organisms cannot be looked upon as an adaptation for the continuation of the individual, but rather for the preservation of a series of individuals. Hence, in this respect also, we cannot profitably compare the individual with a machine, but if we make any comparison we should compare all the individuals that have come from a single one with a machine. In this sense the power of reproduction is a sort of racial regulation. A comparison of this sort is obviously empty of real significance.

The regenerative power of the organism, by means of which it may replace a lost part, or by means of which a piece may become a new whole, is also something not present in machines.

In using a machine for comparison we should not leave out of sight the fact that machines are themselves the work of organisms, and have been made for some purpose useful to the organism. They may perform the same purpose for which we would use our own hands, for they differ from 
parts of the body mainly in that they are made of different compounds having different properties, as the above comparisons have shown. But the regulations of the machine have been added to it by man on account of their usefulness to himself, and are not properties of the material of which the machine itself is composed. This shows, I think, the inappropriateness of making any comparison between these two entirely different things.

If, then, we find the comparison between machines and organisms unprofitable, can we find any other things in inorganic nature that can be better compared with the phenomenon of adaptation of the organism? The following phenomena have been made the subject of comparison from time to time. The bendings, which are gradually made by rivers often lead to a meeting of the loops, so that a direct, new communication is established, and the course of the river is straightened out. The water takes, therefore, a more direct course to the sea. It cannot be said, however, to be of any advantage to the river to straighten its course. Again, a glacier moulds itself to its bed, and gradually moves around obstacles to a lower level, but this adaptation of the glacier to the form of its surroundings cannot be said to be of advantage to the glacier. On the contrary, the glacier reaches so much the sooner a lower level where it is melted.

The unusual case of a solid being lighter than the liquid from which it forms, as seen in the case of ice, has been looked upon as a useful arrangement, since were the reverse the case all rivers and ponds would become solid in winter in cold climates, and the polar regions would become one solid block of ice. But no one will suppose for a moment that there is any relation between the anomalous condition of the lightness of ice, and its relation to the winter freezing of streams, ponds, etc. It has even been suggested that this property of ice was given to it in order that the animals 
living in the water might not be killed, which would be the case if the ice sank to the bottom, but such a method of interpreting physical phenomena would scarcely commend itself to a physicist.

The formation of a covering of oxide over the surface of a piece of iron delays the further process of oxidation, but who will imagine that this property of iron has been acquired in order to prevent the iron from being destroyed by oxygen?

If a piece is broken from a crystal, and the crystal is suspended in a saturated solution of the same substance, new material is deposited over its whole surface, and, as it grows larger, the broken side is completed and the crystal assumes its characteristic form. But of what advantage is it to the crystal whether it is complete or incomplete? In the case of an animal it is of some importance to be able to complete itself after injury, because it can then better obtain the food necessary to keep it alive, or it can better escape its enemies; but this is not the case with the crystal.

In conclusion, therefore, it is obvious that the adaptations of organisms are something peculiar to living things, and their obvious purpose is to maintain the integrity of the individual, or that of the species to which the individual belongs. We are, therefore, confronted with the question as to how this peculiarity has come to be associated with the material out of which living things are made. In subsequent chapters this will be fully discussed, but before we take up this topic, it will be necessary to reach some understanding in regard to the theory of evolution, for the whole subsequent issue will turn upon the question of the origin of the forms of animals and plants living at the present time. 


\section{CHAPTER II}

\section{THE THEORY OF EVOLUTION}

ONE of the most important considerations in connection with the problem of adaptation is that in all animals and plants the individuals sooner or later perish and new generations take their places. Each new individual is formed, in most cases, by the union of two germ-cells derived one from each parent. As a result of this process of intermixing, carried on from generation to generation, all the individuals would tend to become alike, unless something else should come in to affect the result.

So far as our actual experience reaches, we find that the succeeding generations of individuals resemble each other. It is true that no two individuals are absolutely alike, but if a sufficiently large number are examined at a given time, they will show about the same variations in about the same proportionate numbers. Such a group of similar forms, repeat. ing itself in each generation, is the unit of the systematists, and is called a species.

It has been said that within each species the individuals differ more or less from each other, but our experience teaches that in each generation the same kinds of variations occur, and, moreover, that from any one individual there may arise in the next generation any one of the characteristic variations. Certain limitations will have to be made in refrard to this statement, but for the present it will suffice. The law of Biogenesis states that each living thing arises from another living thing: that there is no life without antecedent life, i.c. spontaneous generation does not occur. The 
law is not concerned with the likeness or unlikeness of the different individuals that descend from each other. The theory of -evolution includes the same idea, but in addition it has come to mean nowadays, that there have been changes, as the succeeding generations have arisen. The transmutation theory, and even the descent theory, have come to mean nearly the same thing as the theory of evolution. It is unfortunate that one of these terms cannot be used to signify simply the repetition, generation after generation, of groups of similar indiriduals. The theory of clescent might be used to convey only this idea, but unfortunately it too has come to include also the iclea of change. I shall attempt nevertheless to discriminate between the descent and the transmutation theory, and use the term descent theory when I do not wish to convey the idea of change, and transmutation thcory when I do wish to emphasize this idea.

On the transmutation theory it is assumed that a group (species) may give rise to one or more groups of forms differing from their ancestors; the original group being now replaced by its new kinds of offspring, or the old and the new may remain in existence at the same time. This process repeating itself, each or some of the new groups giving rise in turn to one or more new species, there will be produced a larger group of species having certain similar eharacters which are due to their common descent. Such a group of species is called a genus. The resemblances of these species is accounted for by their common descent; but their differences must be due to those factors that have caused them to depart from the original type. We may now proceed to consider the evidence on which this idea of transmutation rests. 
Evidence in Favor of the Transmutation Theory EVIDENCE FROM CLASSIFICATION AND FROM COMPARATIVE ANATOMY

It does not require any special study to see that there are certain groups of animals and of plants that are more like each other than they are like the members of any other group. It is obvious to every one that the group known as mammals has a combination of characters not found in any other group ; such, for instance, as a covering of hair, mammary glands that furnish milk to the young, and a number of other less distinctive features. These and other common characteristics lead us to put the mammals into a single class. The birds, again, have certain common characters such as feathers, a beak without teeth, the development of a shell around the egg, etc., and on account of these resemblances we put them into another class. Everywhere in the animal and plant kingdoms we find large groups of similar forms, such as the butterflies, the beetles, the annelidan worms, the corals, the snails, the starfishes, etc.

Within each of these groups we find smaller groups, in each of which there are again forms more like each other than like those of other groups. We may call these smaller groups families. Within the families we find smaller groups, that are more like each other than like any other groups in the same family, and these we put into genera. Within the genus we find smaller groups following the same rule, and these are the species. Here we seem to have reached a limit in many cases, for we do not always find within the species groups of individuals more like each other than like other groups. Although we find certain differences between the individuals of a species, yet the differences are often inconstant in the sense that amongst the descendants of any individual there may appear any one of the other variations. If this were the whole truth, it would seem that we had here 
reached the limits of classification, the species being the unit. This, however, is far from being the case, for, in many species we find smaller groups, often confined to special localities. These groups are called varieties.

In some cases it appears, especially in plants, these smaller groups of varieties resemble in many ways the groups of species in other forms, since they breed true to their kind, even under changed conditions. They have been recognized as "smaller species" by a number of botanists.

In this connection a point must be brought up that has played an important rôle in all discussion as to what limits can be set to a species. As a rule it is found that two distinct species cannot be made to cross with each other, i.c. the eggs of an individual of one species cannot be fertilized by spermatozoa derived from individuals of another species; or, at least, if fertilization takes place the embryo does not develop. In some cases, however, it has been found possible to cross-fertilize two distinct species, although the offspring is itself more or less infertile. Even this distinction, however, does not hold absolutely, for, in a few cases, the offspring of the cross is fertile. It cannot be maintained, therefore, that this test of infertility between species invariably holds, although in a negative sense the test may apply, for if two different forms are infertile, inter sc, the result shows that they are distinct species. If they cross they may or may not be good species, and some other test must be used to decide their relation.

We should always keep in mind the fact that the individual is the only reality with which we have to deal, and that the arrangement of these into species, genera, families, etc., is only a scheme invented by man for purposes of classification. Thus there is no such thing in nature as a species, except as a concept of a group of forms more or less alike. In nature there are no genera, families, orders, etc. These are inven. tions of man for purposes of classification. 
Having discovered that it is possible to arrange animals and plants in groups within groups, the question arises as to the meaning of this relation. Have these facts any other significance than that of a classification of geometric figures, or of crystals according to the relations of their axes, or of bodies as to whether they are solids, liquids, or gases, or even whether they are red, white, or blue?

If we accept the transmutation view, we can offer an explanation of the grouping of living things. According to the transmutation theory, the grouping of living things is due to their common descent, and the greater or less extent to which the different forms have diverged from each other. It is the belief in this principle that makes the classification of the biologist appear to be of a different order from that in any other science; and it is this principle that appears to give us an insight into a large number of phenomena.

For example, if, as assumed in the theory, a group of individuals (species) breaks up into two groups, each of these may be supposed to inherit a large number of common characteristics from their ancestors. These characters are, of course, the resemblances, and from them we conclude that the species are related and, therefore, we put them into the same genus. The differences, as has been said, between the species must be explained in some other way; but the principle of classification with which we are here concerned is based simply on the resemblances, and takes no account of the differences between species.

In this argument it has been tacitly assumed that the transformation of one species into another, or into more than one, takes place by adding one or more new characters to those already present, or by changing over a few characters without altering others. But when we come to examine any two species whatsoever, we find that they differ, not only in one or in a few characters, but in a large number of points; perhaps in every single character. It is true that sometimes 
the differences are so small that it is clifficult to distinguish between two forms, but even in such cases the differences, although small, may be as numerous as when they are more conspicuous. If, then, this is what we really find when we carefully examine species of animals or of plants, what is meant when we claim that our classification is based on the characters common to all of the forms that have descended from the same ancestor? We shall find, if we press this point that, in one sense, there is no absolute basis of this sort for our classification, and that we have an unreal system.

If this is admitted, does our boasted system of classification, based as it is on the principle of descent, give us anything fundamentally different from an artificial classification? A few illustrations may make clearer the discussion that follows. If, for example, we take a definition of the group of vertebrates we read: "The group of craniate vertebrates includes those animals known as Fishes, Amphibians, Reptiles, Birds, and Mammals; or in other words, Vertebrates with a skull, a highly complex brain, a heart of three or four chambers, and red blood corpuscles." If we attempt to analyze this definition, we find it stated that the skull is a characteristic of all vertebrates, but if we ask what this thing is that is called skull, we find not only that it is something different in different groups, being cartilaginous in sharks, and composed of bones in mammals, but that it is not even identical in any two species of vertebrates. If we try to define it as a case of harder material around the brain, then it is not something peculiar to the vertebrates, since the brain of the squid is also encased in a cartilaginous skull. What has been said of the skull may be said in substance of the brain, of the heart, and even of the red blood corpuscles.

If we select another group, we find that the birds present a sharply defined class with very definite characters. The definition of the group runs as follows: "Birds are characterized by the presence of feathers, their fore-limbs are 
used for flight, the breast-bone is large and serves for the attachment of the muscles that move the wings; outgrowths from the lungs extend throughout the body and even into the bones and serve as air sacs which make the body more buoyant. Only one aortic arch is present, the right, and the right ovary and oviduct are not developed. The eyes are large and well developed. Teeth are absent. We have here a series of strongly marked characteristics such as distinguish hardly any other class. Moreover, the organization of existing birds is, in its essential features, singularly uniform; the entire class presenting less diversity of structure than many orders of Fishes, Amphibians, and Reptiles." 1 The feathers are the most unique features of birds, and are not found in any other group of the animal kingdom; moreover the plan on which they are formed is essentially the same throughout the group, yet in no two species are the feathers identical, but differ not only in form and proportions, but even in the character of the barbs and hooks for holding the vane together. The modification of the fore-limbs for flight is another characteristic feature; yet in some birds, as the ostrich and kiwi, although the wing has the same general plan as in other birds, it is not used for flight. In the latter it is so small that it does not project beyond the feathers, and in some bircls, as in the penguins, the wings are used only as organs for swimming.

In spite of these differences we have no difficulty in recognizing throughout the group of birds a similarity of plan or structure, modified though it be in a thousand different ways.

Enough has been said to illustrate what is meant by the similarities of organisms on which we base our system of classification. When we conchucle from the statement that all vertebrates have a skull that they owe this to a common descent, we do not mean that a particular structure has been

1 Parker and Haswell: "Text Book of Zoolugy." 


\section{The Theory of Evolution}

handed down as a sort of entailed heirloom, but that the descendants have followed the same plan of structure as that of their ancestors, and have the brain enclosed in a covering of harder material, although this material may not have exactly the same form, or be made of the same substance in all cases. Furthermore while we may recognize that the cartilaginous skull of the shark is simpler in structure than that of the cartilaginous-bony skull of the frog, and that the skull of the frog is simpler than that of the rabbit, yet we should not be justified in stating, except in a metaphorical sense, that something has been added to the skull of the shark to make that of the frog, and something to the latter to make that of the rabbit. On the contrary, while something may have been added, and the plan made more complicated, the skull has also been changed throughout in every single part.

There is another point of some importance to be taken into account in this connection; namely, that each new generation begins life as a single cell or egg. The egg does not contain any preformed adult structures that it hands down unaltered, but it is so constructed that, under constant conditions, the same, or nearly the same, kind of structure is produced. Should something affect the egg, we can imagine that it might form a new combination on the same general plan as that of the old, yet one that differed from the original in every detail of its structure. It is this idea, I believe, that lies at the base of the transmutation theory. On some such assumption as this, and on this alone, can we bring the theory of transmutation into harmony with the facts of observation.

What has been said in regard to individuals as a whole may be repeated also in respect to the study of the single organs. Selecting any one group of the animal or plant kingdom, we find the same organ, or the same combination of organs present in whole groups of forms. We can often 
arrange these organs in definite series passing from the simple to the complex, or, in case of degeneration, in the reverse order. However convenient it may be to study the structure of organisms from this point of view, the artificiality of the procedure will be obvious, since here also the organs of any two species do not cliffer from each other in only one point, but in many, perhaps in all. Therefore to arrange or to compare them according to any one scheme gives only an incomplete idea of their structure. We should apply here the same point of view that we used above in forming a conception of the meaning of the zoological and botanical systems. We must admit that our scheme is only an ideal, which corresponds to nothing real in nature, but is an abstraction based on the results of our experience. It might be a pleasing fancy to imagine that this ideal scheme corresponds to the plan of structure or of organization that is in every egg, and furnishes the basis for all the variations that have come or may come into existence; but we should find no justification whatsocver for believing that our fiction corresponds to any such real thing.

To sum up the discussion: we find that the resemblances of animals and plants can be accounted for on the transmutation theory, not in the way emmonly implied, but in a somewhat different sense. We have found that the resemblances between the different members of a gromp are only of a very general sort, and the structures are not identically the same in any two species - in fact, perhaps in no two individuals. This conclusion, however, does not stand in contradiction to the transmutation hypothesis, because, since each individual begins as an egg which is not a replica of the original adult from which it is derived, there can be no identity, but at most a very close similarity. Admitting, then, that our scheme is an ideal one, we can claim, nevertheless, that on this basis the facts of classification find a legitimate explanation in the transmutation theory. 
THE GEOLOGICAL EVIDENCE

On the theory of descent, as well as on the theory of transmutation, the ancestors of all present forms are supposed to have lived at some time in the past on the surface of the earth. If, therefore, their remains should have been preserved, we should expect on the descent theory to find some, at least, of these remains to be like present forms, while on the transmutation theory we should expect to find most, if not all, of the ancestral forms to be different from the present ones.

The evidence shows that fossil forms are practically all different from living forms, and the older they are the greater the difference from present forms. In general, therefore, it may be said that the evidence is in favor of the transmutation theory. It can scarcely be claimed that the evidence is absolutely conclusive, however probable it may appear, for the problem is complicated in a number of ways.

In the first place, there is convincing evidence that some forms have been entirely exterminated. Other groups have very few living representatives, as is the case in the group containing nautilus, and in that of the crinoids. It is therefore always possible that a given fossil form may represent an extinct line, and may be only indirectly connected with forms alive at the present time. Again the historical record is so broken and incomplete in all but a few cases that its interpretation is largely a question of probability. We can easily conceive that it would be only in very exceptional cases that successive generations of the same form would be buried one above the other, so that we should find the series umbroken. This is evident not only because the conditions that were at one time favorable for the preservation of organic remains might not be favorable at another time, but also because if the conditions remained the same the organisms themselves might also remain unchanged. A new 
form, in fact, would be, $c x$ hypothese, better suited to live in a different environment, and consequently we should not expect always to find its remains in the same place as that occupied by the parent species. This possibility of migration of new forms into a new locality makes the interpretation of the geological record extremely hazardous.

Nevertheless, if the evolution of the entire animal and plant kingdoms had taken place within the period between the first deposits of stratified rocks and the present time, we might still have expected to find, despite the imperfections of the record, sufficient evidence to show how the present groups have arisen, and how they are related to one another. But, unfortunately, at the period when the history of the rocks begins, nearly all the large groups of animals were in existence, and some of them, indeed, as the trilobites and the brachiopods, appear to have reached the zenith of their development.

On the other hand, the subdivisions of the group of vertebrates have evolved during the period known to us. It is true that the group was already formed when our knowledge of it begins, but, from the fishes onwards, the history of the vertebrates is recorded in the rocks. The highest group of all, the mammals, has arisen within relatively modern times. The correctness of the transmutation theory could be as well established by a single group of geological remains as by the entire animal kingdom. Let us, therefore, examine how far the theory is substantiated by the paleontological record of the vertebrates. We find that the earliest vertebrates were fishes, and these were followed successively by the amphibians, reptiles, birds, and mammals, one of the last species of all to appear being man himself. There can be little doubt that this series, with certain limitations to be spoken of in a moment, represents a progressive series beginning with the simpler forms and ending with the more complicated. Even did we not know this geological sequence we would conclude, from 


\section{The Theory of Evolution}

the anatomical evidence alone, that the progression had been in some such order as the geological record shows. The limitation referred to above is this: that while the mammals arose later than the birds, we need not suppose that the mammals arose from the birds, and not even perhaps from the reptiles, or at least not from reptiles like those living at the present day. The mammals may in fact, as some anatomists believe, have come direct from amphibian-like forms. If this is the case, we find the amphibians giving rise on one hand to reptiles and these to birds, and on the other hand to mammals.

This case illustrates how careful we should be in interpreting the record, since two or more separate branches or orders may arise independently from the same lower group. If the manmals arose from the amphibians later than did the reptiles, it would be easy to make the mistake, if the record was incomplete at this stage, of supposing that the mammals had come directly from the reptiles.

That the birds arose as an offshoot from reptile-like forms is not only probable on anatomical grounds, but the geological record has furnished us with forms like archæopteryx, which in many ways appears to stand midway between the reptiles and birds. This fossil, archæopteryx, has a birdlike form with feathered wings, and at the same time has a beak with reptilian teeth, and a long, feathered tail with a core of vertebra.

From another point of view we see how difficult may be the interpretation of the geological record, when we recall that throughout the entire period of evolution of the vertebrates the fishes, amphibians, reptiles, and birds remained still in existence, although they, or some of them, may have at one time given origin to new forms. In fact, all these groups are alive and in a flourishing condition at the present time. The fact illustrates another point of importance, namely, that we must not infer that because a group gives rise to a higher 
one, that it itself goes out of existence, being exterminated by the new form. There may be in fact no relation whatsoever between the birth of a new grcup and the extermination of an old one.

On the transmutation theory we should expect to find not only a sequence of forms, beginning with the simplest and culminating with the more complex, but also, in the beginning of each new group, forms more or less intermediate in structure. It is claimed by all paleontologists that such forms are really found. For example, transitional forms between the fishes and the amphibia are found in the group of dipnoans, or lung-fishes, a few of which have survived to the present day. There are many fossil forms that have characters between those of amphibians and reptiles, which if not the immediate ancestors of the reptiles, yet show that at the time when this group is supposed to have arisen intermediate forms were in existence. The famous archæopteryx remains have been already referred to above, and it appears in this case that we have not only an intermediate form, but possibly a transitional one. In the group' of mammals we find that the first forms to appear were the marsupials, which are undoubtedly primitive members of the group.

The most convincing evidence of transmutation is found in certain series of forms that appear quite complete. The evolution of the horse series is the most often eited. As this case will be discussed a little later, we need not go into it fully here. It will suffice to point out that a continuous series of forms has been found, that comnect the living horses having a single toe through three-toed, with the fivetoed horses. Moreover, and this is important, this series shows a transformation not only in one set of structures, but in all other structures. The fossil horses with three toes are found in the higher geological layers, and those with more toes in the decper layers progressively. In some cases, at 
least, the fossils have been found in the same part of the world, so that there is less risk of arranging them arbitrarily in a series to fit in with the theory.

\section{EVIDENCE FROM DIRECT OBSERVATION AND EXPERIMENT}

Within the period of human history we do not know of a single instance of the transformation of one species into another one, if we apply the most rigid and extreme tests used to distinguish wild species from each other. ${ }^{1}$ It may be claimed that the theory of descent is lacking, therefore, in the most essential feature that it needs to place the theory on a scientific basis. This must be admitted. On the other hand, the absence of direct observation is not fatal to the hypothesis, for several reasons. In the first place, it is only within the last few hundred years that an accurate record of wild animals and plants has been kept, so that we do not know except for this period whether any new species have appeared. Again, the chance of observing the change might not be very great, especially if the change were sudden. We would simply find a new species, and could not state where it had come from. If, on the other hand, the change were very slow, it might extend over so many years that the period would be beyond the life of an individual man. In only a few cases has it been possible to compare ancient pictures of animals and plants with their prototypes living at the present time, and it has turned out in all cases that they are the same. But these have been almost entirely domesticated forms, where, even if a change had been found, it might have been ascribed to other factors. In other cases, as in the mummified remains of a few Egyptian wild animals (which have also been found to be eractly like the same animals living at the present day),

1 The transformation of "smaller species," described by De Vries, will be described in a later chapter. 
it was pointed out by Geoffroy Saint-Hilaire that, since the conditions of the Egyptian climate are the same to-day as they were two thousand years ago, there is no reason to expect any change would have taken place. But waiving this assumption, we should not forget that the theory of evolution does not postulate that a change must take place in the course of time, but only that it may take place sometimes.

The position that we have here taken in regard to the lack of evidence as to the transformation of species is, perhaps, extreme, for, as will be shown in some detail in later chapters, there is abundant evidence proving that species have been seen to change greatly when the conditions surrounding them have been changed; but never, as has been stated, so far, or rather in such a way, that an actual new species that is infertile with the original form has been produced. Whether, after all, these changes clue to a change in the environment are of the kind that makes new species, is also a question to be discussed later.

The experimental evidence, in favor of the transformation of species, relates almost entirely to domesticated forms, and in this case the conscious agency of man seems, in some cases, to have played an important part; but here, even with the aid of the factor of isolation, it cannot be claimed that a single new species has been produced, although great changes in form have been effected. It is clear, therefore, that we must, at present, rely on other data, less satisfactory in all respects, to establish the probability of the theory of transformation.

\section{MODERN CRITICISM OF THE THEORY OF EVOLUTION}

Throughout the whole of the nineteenth century a steady fire of criticism was directed against the theory of evolution; the names of Cuvier and of Louis Agassiz stand out preëmi- 
nent in this connection, yet the theory has claimed an ever increasing number of adherents, until at the present time it is rare to find a biologist who does not accept in one form or another the general principle involved in the theory. The storm of criticism aroused by the publication of Darwin's "Origin of Species," was directed more against the doctrine of evolution than against Darwin's argument for natural selection. The ground has been gone over so often that there would be little interest in going over it again. It will be more profitable to turn our attention to the latest attack on the theory from the ranks of the zoologists themselves.

Fleischmann, in his recent book, " Die Descendenztheorie," has made a new assault on the theory of evolution from the three stanclpoints of paleontology, comparative anatomy, and embryology. His general method is to try to show that the recognized leaders in these different branches of biology have been led to express essentially different views on the same questions, or rather have compromised the doctrine by the examples they have given to illustrate it. Fleischmann is fond of bringing together the antiquated and generally exaggerated views of writers like Haeckel, and contrasting them with more recent views on the same subject, without making sufficient allowances for the advances in knowledge that have taken place. He selects from each field a few specific examples, by means of which he illustrates the weakness, and ever, as he believes, the falsity of the deductions drawn for the particular case. For example, the plan of structure of the vertebrates is dealt with in the following way: In this group the limbs, consisting typically of a pair of forelegs and a pair of hind-legs, appear under the form of cylindrical outgrowths of the body. In the salamander. in the turtle, in the dog, the cylindrical legs, supporting the body and serving to support it above the ground, are used also for progression. The general purpose to which 
the limbs are put as organs of locomotion has not interfered with an astonishing number of varieties of structure, adapted to different conditions of existence, such as the short legs used for creeping in salamanders, lizards, turtles, crocodiles; the long and thin legs of good rumners, as the hoofed animals; the mobile legs of the apes used for climbing; and the parachute legs of some squirrels used for soaring. Even more striking is the great variety of hands and feet, as seen in the flat, hairy foot of the bear; the fore-foot of the armadillos, carrying long, sickle-shaped claws; the digging foot of the mole; the plump foot of the elephant, ending in a broad, flat pad with nails around the border, and without division into fingers; the hand of man and of the apes ending with fine and delicate fingers for grasping. To have discovered a general plan of structure running through such a great variety of forms was proclaimed a triumph of anatomical study. ${ }^{1}$

A study of the bony structure of the limb shows that typically it consists of a single proximal bone (the humerus in the upper arm, the femur in the thigh), followed by two bones ruming parallel to each other (the radius and una in the arm and the tibia and fibula in the shank); these are succeeded in the arm by the two series of carpal bones, and in the leg by the two series of tarsal bones, and these are followed in each by five longer bones (the metacarpals and metatarsals), and these again by the series of long bones that lie in the fingers and toes. Despite the manifold varicty of forms, Fleischmann admits that both the hind- and the fore-limbs are constructed on the same plan throughout the vertebrates. Even forms like the camel, in which there are fewer terminal bones, may be brought into the same category by supposing a reduction of the bones to have taken place, so that three of the digits have been lost. In the leg of the pig and of the reindeer, even a greater reduction may

\footnotetext{
1 This paragrapl is a free translation of Fleischmann's text.
} 
be supposed to have taken place. Fleischmann points out that these facts were supposed to be in full harmony with the theory of descent.

The analysis of the origin of the foot of the horse gave even better evidence, it was claimed, in favor of the theory. The foot consists of a single series of bones corresponding to the middle finger and toe. When, as sometimes happens, individual horses are found in which in addition to the single middle finger two smaller lateral fingers with small hoofs appear, the followers of the descent theory rejoiced to be able to bring this forward as a confirmation of their doctrine. The occurrence was explained as a sporadic return to an ancestral form. The narve exposition of the laws of inheritance that were supposed to control such phenomena was accepted without question. And when finally a large number of fossil remains were found by paleontologists, remains showing a gradual increase in the middle finger, and a decrease in size of the latcral fingers, - it was supposed that the proof was complete; and anatomists even went so far as to hold that the original ancestor of the horse was a five-fingered animal.

This same law of type of structure was found to extend to the entire vertebrate series, and the only plausible explanation appeared to be that adopted by Darwin and his followers, namely, that the resemblance is the result of the blood-relationship of the different forms. But a simple comparison of the skeleton of the limbs if carried out without theoretical prejudice would show, Fleischmann thinks, that there is only a common style, or plan of structure, for the vertebrates. This anatomical result has about the same value as the knowledge of the different styles of historical architecture - that, for instance, all large churches of the Gothic period have certain general principles in common. The believers in the theory of descent have, however, he thinks, gone beyond the facts, and have concluded that the 
common plan in animals is the consequence of a common descent. "I cannot see the necessity for such a conclusion, and I certainly should unhesitatingly deny that the common plan of the Gothic churches depended on a common architect. The illustration is, however, not perfect, because the influence of the medireval school of stone-cutters on its wandering apprentices is well known."

Fleischmann adds that if the descent theory is true we should expect to find that if a common plan of structure is present in one set of organs, as the limbs, it should be present in all other organs as well, but he does not add that this is generally the case.

The weakness of Fleischmann's argument is so apparent that we need not attempt an elaborate refutation. When he says there is no absolute proof that the common plan of structure must be the result of blood-relationship, he is not bringing a fatal argument against the theory of descent, for no one but an enthusiast sees anything more in the explanation than a very probable theory that appears to account for the facts. To demand an absolute proof for the theory is to ask for more than any reasonable adrocate of the descent theory claims for it. As I have tried to show in the preceding pages, the evidence in favor of the theory of descent is not absolutely demonstrative, but the theory is the most satisfactory one that has as yet been advanced to account for the facts. Fleischmann's reference to the common plan of structure of the Gothic churches is not very fortumate for his purpose, since he admits himself that this may be the result of a common tradition handed down from man to nan, a sort of continuity that is not very dissimilar in principle from that implied in the descent theory; in the latter the continuity of substance taking the place of the tradition in the other. Had the plan for each, or even for many of the churches, originated independently in the mind of each architect, then the similarity in style would have to be accounted for by a 
different sort of principle from that involved in the theory of descent; but as a matter of fact the historical evidence makes it probable that similar types of architecture are largely the result of imitation and tradition. Certain variations may have been added by each architect, but it is just the similarity of type or plan that is generally supposed to be the outcome of a common tradition.

Fleischmann's attempt in the following chapter to belittle Gegenbaur's theory of the origin of the five-fingered type of hand from a fin, like that of a fish, need not detain us, since this theory is obviously only a special application which like any other may be wrong, without in the least injuring the general principle of clescent. That all phylogenetic questions are hazardous and difficult is only too obvious to any one familiar with the literature of the last thirty years.

Fleischmann devotes a long chapter to the geological evidences in connection with the evolution of the horse, and attempts to throw ridicule on the conclusions of the paleontologists by emphasizing the differences of opinion that have been advanced in regard to the descent of this form. After pointing out that the horse, and its few living relatives, the ass and the zebra, are unique in the mammalian series in possessing a single digit, he shows that by the discovery of the fossil horses the group has been simply enlarged, and now includes horses with one, three, and five toes. The discovery of the fossil forms was interpreted by the advocates of the descent theory as a demonstration of the theory. The series was arranged by paleontologists so that the five-toed form came first, then those with three and one tree, the last represented by the living horses. But the matter was not so simple, Fleischmann points out, as it appeared to be to the earlier writers, for example to Haeckel, Huxley, Leidy, Cope, Marsh. Different authors came to express different opinions in regard to the genealogical connection between the fossil forms. Several writers have tried to show 
that the present genus, Equus, has not had a single line of descent, but have supposed that the European horses and the original American horses had different lines of ancestry, which may have united only far back in the genus Epihippus. Fleischmann points out that the arrangement of the series is open to the criticism that it is arbitrary, and that we could equally well make up an analogous series beginning with the five-fingered hand of man, then that of the dog with the thumb incompletely developed, then the four-fingered hindfoot of the pig without a big toe and with a weak second and fifth digit, then the foot of the camel with only two toes, and lastly the foot of the horse with only one toe. It sounds strange that Fleischmann should make such a trivial reply as this, and deliberately ignore the all-important evidence with which he is, of course, as is every zoologist, perfectly conversant. Not only are there a hundred other points of agreement in the horse series, but also the geological sequence of the strata, in which some at least of the series have been found, shows that the arrangement is not arbitrary, as he implies.

Fleischmann then proceeds to point out that when the eridence from other parts of the anatomy is taken into account, it becomes evident that all the known fossil remains of horses cannot be arranged in a single line, but that there are at least three families or groups recognizable. Many of these forms are known only from fragments of their skeletons - a few teeth, for instance, in the case of Merohippus, which on this evidence alone has been placed at the uniting point of two series. At present about eight different species of living horses are recognized by zoologists, and paleontological evidence shows only that many other species have been in existence, and that even three- and onetoed forms lived together at the same time.

Fleischmann also enters a protest against the ordinary arrangement of the fossil genera Eo-, Oro-, Meso-, Mero- 
hippus in a series, for these names stand not for single species, but for groups containing no less than six species under Protohippus, fourteen under Equus, twelve under Mesohippus, and twenty under Hipparion. Fleischmann concludes: "The descent of the horses has not been made out with the precision of an accurate proof, and it will require a great deal of work before we get an exact and thorough knowledge of the fossil forms. What a striking contrast is found on examination between the actual facts and the crude hopes of the apostles of the descent theory! . .."

In so far as this criticism of Fleischmann's applies to the difficulties of determining the past history of the horse, it may be granted that he has scored a point against those who have pretended that the evidence is simple and conclusive; but we should not fail to remember that this difficulty has been felt by paleontologists themselves, who have been the first to call attention to the complexity of the problem, and to the difficulties of finding out the actual ancestors of the living representative of the series. And while we may admit that the early enthusiasts exaggerated, unintentionally, the importance of the few forms known to them, and went too far in supposing that they had found the actual series of ancestors of living horses, yet we need not let this blind us to the importance of the facts themselves. Despite the fact that it may be difficult and, perhaps, in most cases, impossible, to arrange the fossil forms in their relations to one another and to living forms, yet on an unprejudiced view it will be clear, I think, that so far as the evidence goes it is in full harmony with the theory of descent. This is especially evident if we turn our attention to a part of the subject that is almost entirely ignored by Fleischmann, and yet is of fundamental importance in judging of the result. The series of forms beginning with the five-toed horses and ending with those having a single toe has not been brought together haphazard, as Fleischmann's comparison might lead one to suppose, but 
the five-fingered forms are those from the older rocks, and the three-toed forms from more recent layers. The value of this kind of evidence might have been open to greater doubt had the series been made up of forms found scattered over the whole world, for it is well known how difficult it is to compare in point of time the rocks of different continents. But in certain parts of the world, especially in North America, series of fossil horses have been found in sedimentary deposits that appear to be perfectly continuous. This series, by itself, and without regard to the point as to whether in other parts of the world other series may exist, shows exactly those results which the theory of descent postulates, and we find here, in all probability, a clirect line of descent. While it may be freely admitted that no such series can demonstrate the theory of descent with absolute certainty, yet it would be folly to disregard evidence as clear as this.

- In regard to the other point raised by Fleischmann concerning the large number of species of fossil horses that have existed in past times, it is obvious that while this greatly increases the difficulty of the paleontologist it is not an objection to the descent theory. In fact, our experience with living species would lead us to expect that many types have been represented at each geological period by a number of related species that may have inhabited the same country. On the descent theory, one species only in each geological period could have been in the line of descent of the present species of horse. The difficulty of determining which species (if there were several living in a given epoch) is the ancestor of the horse is increased, but this is not in itself an objection to the theory.

The descent of birds from flying reptiles is used by Fleischmann as another point of attack on the transmutation theory. The theory postulates that the birds have come from ancestors whose fore-legs have been changed into highly specialized wings. The long vertebrated tail of the 
ancestral form is supposed to have become very short, and long feathers to have grown out from its stump which act as a rudder during flight. Flying reptiles with winged forelegs and a long vertebrated tail have been actually found as fossil remains, as seen in the pterodactyls and in the famous archeopteryx. The latter, which is generally regarded either as the immediate ancestor of living birds, or at least as a closely similar form, possessed a fore-leg having three fingers ending in claws, and feathers on the forearm similar to those of modern birds. It had a long tail, like that of a lizard, but with well-developed feathers along its sides. It had pointed teeth in the horn-covered jaws. Fleischmann proceeds to point out that the resemblance of the hand of archreopteryx to that of the reptiles is not very close, for two fingers are absent as in modern birds. The typical form of the foot is that of the bird, and is not the simple reptilian type of structure. Feathers and not scales cover the body, and give no clew as to how the feathers of birds have arisen. He concludes, therefore, that archæopteryx, having many true birdlike characters, such as feathers, union of bones in the foot, etc., has other characters not possessed by living birds, namely, a long, vertebrated tail, a flat breastbone, biconcave vertebræe, etc. Therefore, it cannot be regarded as an intermediate form. Fleischmann does not point out that it is just these characters that would be postulated on the descent theory for the ancestor of the birds, if the latter arose from reptiles. Even if it should turn out that archæopteryx is not the immediate forefather of living birds, yet the discovery that a form really existed intermediate in many characters between the reptiles and the birds is a gain for the transmutation theory. It is from a group having such characters that the theory postulates that the birds have been evolved, and to have discovered a member of such a group speaks directly and unmistakably in favor of the probability of the transmutation theory. 
Fleischmann again fails to point out that the geological period in which the remains of archeopteryx were found, is the one just before that in which the modern group of bircls appeared, and, therefore, exactly the one in which the theory demands the presence of intermediate forms. This fact adds important evidence to the view that looks upon archxopteryx as a form belonging to a group from which living birds have arisen. That a number of recent palcontologists believe archaopteryx to belong to the group of birds, rather than to the reptiles, or to an intermediate group, does not in the least lessen its importance, as Fleischmann pretends it does, as a form possessing a number of reptilian characters, such as the transmutation theory postulates for the early ancestors of the birds.

The origin of the mammalian phylum serves as the text for another attack on the transmutation theory. Fleischmann points out that the discovery of the monotremes, including the forms ornithorhynchus and echidna, was hailed at first as a demonstration of the supposed descent of the mammals from a reptilian ancestor. The special points of resemblance between ornithorhynchus and reptiles and birds are the complete fusion of the skull bones, the great development of the vertebre of the neck region, certain similarities in the shoulder girdle, the paired oviducts opening independently into the last part of the digestive tract (cloaca), and the presence of a parehment-like shell around the large, yolkbearing egg. These are all points of resemblance to reptiles and birds, and were interpreted as intermediate stages between the latter groups and the group of mammals. In addition to these intermediate characters, omithorhynchus possesses some distinctive, mammalian features - mammary glands and hair, for instance. Fleischmann takes the ground, in this case, that there are so many points of difference between the monotremes and the higher mammals, that it is impossible to see how from forms like these the higher 
groups could have arisen, and that ornithorynchus cannot be placed as an intermediate form, a link between saurians and mammals, as the followers of the transmutation theory maintain. He shows, giving citations, that anatomists themselves are by no means in accord as to the exact position of ornithorhynchus in relation to the higher forms.

In reply to this criticism, the same answer made above for archæopteryx may be repeated here, namely, that because certain optimists have declared the monotremes to be connecting forms, it does not follow that the descent theory is untrue, and not even that these forms do not give support to the theory, if in a less direct way. I doubt if any living zoologist regards either ornithorhynchus or echidna as the ancestral form from which the mammals have arisen. But on the other hand it may be well not to forget that these two forms possess many characters intermediate between those of mammals and reptiles, and it is from a group having such intermediate characters that we should expect the mammals to have arisen. These forms show, if they show nothing else, that it is possible for a species to combine some of the characters of the reptiles with those of the mammals; and the transmutation theory does no more than postulate the existence at one time of such a group, the different species of which may have differed in a number of points from the two existing genera of monotremes.

The origin of lung-bearing vertebrates from fishlike ancestors, in which the swim-bladder has been changed into lungs, has been pointed to by the advocates of the transmutation theory as receiving confirmation in the existence of animals like those in the group of dipnoan fishes. In these animals both gills and a swim-bladder, that can be used as a lung, are present; and through some such intermediate forms it is generally supposed that the lung-bearing animals have arisen. Fleischmann argues, however, that, on account of certain trivial differences in the position of the duct of the swimbladder in living species, the supposed comparison is not to 
the point; but the issue thus raised is too unimportant to merit further discussion. Leaving aside also some even more doubtful criticisms which are made by Fleischmann, and which might be added to indefinitely without doing more than showing the credulity of some of the more ardent followers of the transmutation theory, or else the uncertainty of some of the special applications of the theory, let us pass to Fleischmann's criticism of the problem of development. ${ }^{1}$

With fine scorn Fleischmann points to the crudity of the ideas of Oken and of Haeckel in regard to the embryology (or the ontogeny) repeating the ancestral history (or the phylogeny). We may consider briefly (since we devote the next chapter almost entirely to the same topic) the exceptions to this supposed recapitulation, which Fleischmann has brought together. The young of beetles, flies, and butterflies creep out of the egg as small wormlike forms of apparently simple organization. They have a long body, composed of a series of rings; the head is small and lacks the feelers, and often the faceted eyes. The wings are absent, and the legs are short. At first sight the larva appears to resemble a worm, and this led Oken to conclude that the insects appear first in the form of their ancestors, the segmented worms. If we examine the structure of the larva more carefully, we shall find that there are a great many differences between it and the segmented worms; and that even the youngest larva is indeed a typical insect. The trachere, so characteristic of the group of insects, are present, the structure of the digestive tract with its Malpighian tubes, the form of the heart, the structure of the head, as well as the blastema of the reproductive organs, show in the youngest larva the type of the insects. In other words the body of the caterpillar is formed on exactly the same fundamental plan as that of the butterfly.

The long argument of Fleischmann in regard to the origin of the freshwater snails, as illustrated by the planorbis series, and also the origin of the nautiloid group, has heen recently dealt with fully ly Plate, and, therefore, need not be considered here. 
In regard to the larval forms of other groups we find the same relations, as, for example, in the amphibians. The young of salamanders, toads, and frogs leave the egg not in the completed form, but as small tadpoles adapted to life in the water. A certain resemblance to fish cannot be denied. They possess a broad tail, gills (rich in blood vessels) on each side of the neck, and limbs are absent for a long time. These are characters similar to those of fish, but a more careful anatomical examination destroys the apparent resemblance. The superficial resemblances are due to adaptation to the same external conditions.

Fleischmann ridicules the idea that the young chick resembles at any stage an adult, ancestral animal; the presence of an open digestive tract shows how absurd such an iclea is. The obvious contradiction is explained away by embryologists, by supposing that the ancestral adult stages have been crowded together in order to shorten the period of development; and that, in addition, larval characters and provisional organs have appeared in the embryo itself, which confuse and crowd out the ancestral stages.

In regard to the presence of gill-slits in the embryo of the higher vertebrates, in the chick, and in man, for example, Fleischmann says: "I cannot see how it can be shown by exact proof that the gill-slits of the embryos of the higher vertebrates that remain small and finally disappear could once have had the power of growing into functional slits." With this trite comment the subject is dismissed.

On the whole, Fleischmann's attack cannot be regarded as having seriously weakened the theory of evolution. He has done, nevertheless, good service in recalling the fact that, however probable the theory may appear, the evidence is indirect and exact proof is still wanting. Moreover, as I shall attempt to point out in the next chapter, we are far from having arrived at a satisfactory idea of how the process has really taken place. 


\section{CHAPTER III}

\section{THE THEORY OF EYOLUTION (Continued) \\ The Evidence from Embriology}

THE RECAPITULATION THEORY

A $\mathrm{T}$ the close of the eighteenth, and more definitely at the beginning of the nineteenth, century a number of naturalists called attention to the remarkable resemblance between the embryos of higher animals and the adult forms of lower animals. This idea was destined to play an important rôle as one of the most convincing proofs of the theory of evolution, and it is interesting to examine, in the first place, the evidence that suggested to these earlier writers the theory that the embryos of the higher forms pass through the adult stages of the lower animals.

The first definite reference ${ }^{1}$ to the recapitulation view that I have been able to find is that of Kielmeyer in 1793, which was inspired, he says, by the resemblance of the tadpole of the frog to an adult fish. ${ }^{2}$ This suggested that the embryo of higher forms corresponds to the adult stages of lower ones. He adds that man and birds are in their first stages plantlike.

Oken in I 805 gave the following fantastic account of this relation: "Each animal 'metamorphoses itself' through all animal forms. The frog appears first under the form of a mollusk in order to pass from this stage to a higher one.

1 The earlier references of a few embryologists are tou vague to have any hearing on the suljject.

2 Autenrieth in 1707 makes the briefest possible reference to some such principle in speaking of the way in which the nose of the embryo cluses. 
The tadpole stage is a true snail; it has gills which hang free at the sides of the body as is the case in (nio pictornm. It has even a byssus, as in Mytilus, in order to cling to the grass. The tail is nothing else than the foot of the snail. The metamorphosis of an insect is a repetition of the whole class, scolopendra, oniscus, julus, spider, crab."

Walther, in I 808 , said: "The human fotus passes through its metamorphosis in the cavity of the uterus in such a way that it repeats all classes of animals, but, remaining permilnently in none, develops more and more into the innate human form. First the embryo has the form of a worm. It reaches the insect stage just before its metamorphosis. The origin of the liver, the appearance of the different secretions, etc., show clearly an advance from the class of the worm into that of the mollusk."

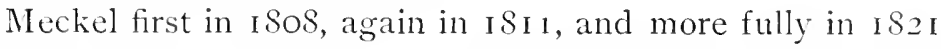
made much more definite comparisons between the embryos of higher forms and the adult stages of lower groups. He held that the embryo of higher forms, before reaching its complete development, passes through many stages that correspond to those at which the lower animals appear to be checked through their whole life. In fact the embryos of higher animals, the mammals, and especially man, correspond in the form of their organs, in their number, position, and proportionate size to those of the animals standing below them. The skin is at first, and for a considerable period of embryonic life, soft, smooth, hairless, as in the zoophytes, medusæ, many worms, mollusks, fishes, and even in the lower amphibians. Then comes a period in which it becomes thicker and hairy, when it corresponds to the skin of the higher animals. It should be especially noted here, that the foetus of the negro is more hairy than that of the European.

The muscular system of the embryo, owing to its lack of union in the ventral wall, corresponds to the muscles of the shelled, headless mollusks, whose mantle is open in the same 
region. Meckel compares the bones of the higher vertebrates with the simpler bones of the lower forms, and even with the cartilages of the cephalopod. He points out that in the early human embryo the nerve cord extends the whole length of the spinal canal. He compares the simple heart of the embryo with that of worms, and a later stage, when two chambers are present, with that of the gasteropod mollusk. The circulation of the blood in the placenta recalls, he says. the circulation in the skin of the lower animals. The lobulated form of the kidney in the human embryo is compared with the adult condition in the fishes and amphibians. The internal position of the reproductire organs in the higher mammals recalls the permanent position of these organs in the lower animals. The posterior end of the body of the human embryo extends backwards as a tail which later disappears.

Some of these comparisons of Meckel sound rery absurd to us nowadays, especially his comparison between the embryos of the higher vertebrates, and the adults of worms. crustaceans, spiders, snails, bivalve mollusks, cephalopods, etc. On the other hand, many of these comparisons are the same as those that are to be found in modern text-books on embryology; and we may do well to ask ourselves whether these may not sound equally absurd a hundred years hence. Why do some of Meckel's comparisons seem so narre, while others have a distinctly molern flavor? In a word, can we justify the present belief of some embryologists that the embryos of higher forms repeat the adult stages of lower members of the same group? It is important to observe that up to this time the comparison had always been made between the embryo of the higher form and the adult forms of existing lower animals. The theory of erolution had, so far, had no influence on the interpretation that was later given to this resemblance.

Von Baer opposed the theory of recapitulation that had 


\section{The Theory of Evolution}

become current when he wrote in I828. According to Von Baer, the more nearly related two animals are, or rather the more nearly similar two forms are (since Von Baer did not accept the idea of evolution), the more nearly alike is their development, and so much longer in their development do they follow in the same path. For example two similar species of pigeons will follow the same method of development up to almost the last stage of their formation. The embryos of these two forms will be practically identical until each produces the special characters of its own species. On the other hand two animals belonging to different families of the same phylum will have only the earlier stages in common. Thus, a bird and a mammal will have the first stages similar, or identical, and then diverge, the mammal adding the higher characters of its group. The resemblance is between corresponding embryonic stages and not between the embryo of the mammal and the adult form of a lower group.

Von Baer was also careful to compare embryos of the same phylum with each other, and states explicitly that there are no grounds for comparison between embryos of different groups. ${ }^{1}$

We shall return again to Yon Baer's interpretation and then discuss its value from our present point of view.

Despite the different interpretation that Von Baer gave to this doctrine of resemblance the older view of recapitulation continued to dominate the thoughts of embryologists throughout the whole of the nineteenth century.

Louis Agassiz, in the Lowell Lectures of 1848 , proposed for the first time the theory that the embryo of higher forms resembled not so much lower adult animals living at the present time, as those that lired in past times. Since Agassiz himself did not accept the theory of evolu-

${ }^{1}$ In one place Von Baer raises the question whether the egg may not be a form common to all the phyla. 
tion, the interpretation that he gave to the recapitulation theory did not have the importance that it was destined to have when the animals that lived in the past came to be looked upon as the ancestors of existing animals. ${ }^{1}$ But with the acceptation of the theory of evolution, which was largely the outcome of the publication of Darwin's "Origin of Species" in I859, this new interpretation immediately blossomed forth. In fact, it became almost a part of the new theory to believe that the embryo of higher forms recapitulated the series of ancestral adult forms through which the species had passed. The one addition of any importance to the theory that was added by the Darwinian school was that the history of the past, as exemplified by the embryonic development, is often falsified.

Let us return once more to the facts and see which of them are regarded at present as demanding an explanation. These facts are not very numerous and yet sufficiently apparent to attract attention at once when known.

The most interesting case, and the one that has most often attracted attention, is the occurrence of gill-clefts in the embryos of reptiles, birds, and mammals. These appear on each side of the neck in the very early embryo. Each is formed by a vertical pouch, that grows out from the wall of the pharynx until it meets the skin, and, fusing with the latter, the walls of the pouch separate, and a cleft is formed. This vertical cleft, placing the cavity of the pharynx in communication with the outside, is the gill-slit. Similar openings in adult fishes put the pharynx in communication with the exterior, so that water taken through the mouth passes out at the sides of the neck between the gill filaments that border the gill-slits. In this way the blood is aerated. The number of gill-slits that are found in the embryos of different groups

1 Carl Vogt in 1 \$. 2 suggested that fossil species, in their historical succession, pass through changes similar to those which the embryos of living forms undergo. 


\section{The Theory of Evolution}

of higher vertebrates, and the number that open to the exterior are variable; but the number of gill-openings that are present in the adults of lower vertebrates is also variable. No one who has studied the method of development of the gill-slits in the lower and higher vertebrates will doubt for a moment that some kind of relation must subsist between these structures.

In the lowest adult form of the vertebrates, amphioxus, the gill-system is used largely as a sieve for procuring food, partly also, perhaps, for respiration. In the sharks, bony fishes, and lower amphibians, water is taken in through the mouth, and passes through the gill-slits to the exterior. As it goes through the slits it passes over the gills, that stand like fringes on the sides of the slits. The blood that passes in large quantities through the gills is aerated in this way. In the embryos of the higher vertebrates the gill-slits may appear even before the mouth has opened, but in no case is there a passage of water through the gill-slits, nor is the blood aerated in the gill-region, although it passes through this part on its way from the heart to the dorsal side of the digestive tract. It is quite certain that the gill-system of the embryo performs no respiratory function. ${ }^{1}$

In the higher amphibians, the frogs for example, we find an interesting transition. The young embryo, when it emerges from the egg-membranes, bears three pairs of external gills that project from the gill-arches into the surrounding water. Later these are absorbed, and a new system of internal gills, like those of fishes, develops on the gill-arches. These are used throughout the tadpole stage for respiratory purposes. When the tadpole is about to leave the water to become a frog, the internal gills are

1 This statement is not intended to prejudice the question as to whether the presence of the gill-slits and arches may be essential to the formation of other organs. 
also absorbed and the gill-clefts close. Lungs then develop which become the permanent organs of respiration.

There are two points to be noticed in this connection. First, the external gills, which are the first to develop, do not seem to correspond to any permanent adult stage of a lower group. Second, the transition from the tadpole to the frog can only be used by way of analogy of what is supposed to have taken place ancestrally in the reptiles, birds, and mammals, since no one will maintain that the frogs represent a group transitional between the amphibians and the higher forms. However, since the salamanders also have gills and gill-slits in the young stages, and lose them when they leave the water to become adult land forms, this group will better serve to illustrate how the gill-system has been lost in the higher forms. Not that in this case either, need we suppose that the forms living to-day represent ancestral, transitional forms, but only that they indicate how such a remarkable change from a gill-breathing form, living in the water, might become transformed into a lung-breathing land form. Such a change is supposed to have taken place when the ancestors of the reptiles and the mammals left the water to take up their abode on the land.

The point to which I wish to draw especial attention in this connection is that in the higher forms the gill-slits appear at a very early stage; in fact, as early in the mammal as in the salamander or the fish, so that if we suppose their appearance in the mammal is a repetition of the adult amphibian stage, then, since this stage appears as early in the development of the mammal as in the amphibians themselves, the conclusion is somewhat paradoxical.

The history of the notochord in the vertebrate series gives an interesting parallel. In amphioxus it is a tough and firm cord that extends from end to end of the body. On each side of it lie the plates of muscles. It appears at a very early stage of development as a fold of the upper wall of 
the digestive tract. In the cartilaginous fishes the notochord also appears at a very early stage, and also from the dorsal wall of the digestive tract. In later embryonic stages it becomes surrounded by a cartilaginous sheath, or tube, which then segments into blocks, the vertebræ. The notochord becomes partially obliterated as the centra of the vertebra are formed, but traces of it are present even in adult stages. In the lower amphibians the notochord arises also at an early stage over and perhaps, in part, from the dorsal wall of the digestive tract. It is later almost entirely obliterated by the development of the vertebre. These vertebræ first appear as a membraneous tube which breaks up into cartilaginous blocks, and these are the structures around and in which the bone develops to form the permanent vertebræ.

In higher forms, reptiles, birds, and mammals, the notochord also appears at the very beginning of the development, but it is not certain that we can call the material out of which it forms the dorsal wall of the archenteron (the amphibians giving, perhaps, intermediate stages). It becomes surrounded by continuous tissue which breaks up into blocks, and these become the bases of the vertebræ. The notochord becomes so nearly obliterated in later stages that only the barest traces of it are left either in the spaces between, or in, the vertebræ.

In this series we see the higher forms passing through stages similar at first to those through which the lower forms pass; and it is especially worthy of note that the embryo mammal begins to produce its notochord at the very beginning of its development, at a stage, in fact, so far as comparison is possible, as early as that at which the notochord of amphioxus develops.

The development of the skull gives a somewhat similar case. The skulls of sharks and skates are entirely cartilaginous and imperfectly enclose the brain. The ganoids 
have added to the cartilaginous skull certain plates in the dermal layer of the skin. In the higher forms we find the skull composed of two sets of bones, one set developing from the cartilage of the first-formed cranium, and the other having a more superficial origin; the latter are called the membrane bones, and are supposed to correspond to the dermal plates of the ganoids.

In the development of the kidneys, or nephridia, we find, perhaps, another parallel, although, owing to recent discoveries, we must be very cautious in our interpretation. As yet, nothing corresponding to the nephridia of amphioxus has been discovered in the other vertebrates. Our comparison must begin, therefore, higher up in the series. In the sharks and bony fishes the nephridia lie at the anterior end of the body-cavity. In the amphibia there is present in the young tadpole a pair of nephridial organs, the head-kidneys, also in the anterior end of the body-eavity. Later these are replaced by another organ, the permanent mid-kidney, that develops behind the head-kidney. In reptiles, birds, and mammals a third nephridial organ, the hind-kidney, derelops later than and posterior to the mid-kidney, and becomes the permanent organ of excretion. Thus in the development of the nephridial system in the higher forms we find the same sequence, more or less, that is found in the series of adult forms mentioned above. The anterior end of the kidney develops first, then the middle part, and then the most posterior. The anterior part clisappears in the amphibians, the anterior and the middle parts in the birds and mammals, so that in the latter groups the permanent kidney is the hindkiclney alone.

The formation of the heart is supposed to offer certain parallels. Amphioxus is without a definite heart, but there is a ventral blood vessel beneath the pharymx, which sends blood to the gill-system. This blood vessel corresponds in position to the heart of other vertebrates. In sharks we find a thick- 
walled muscular tube below the pharynx; the blood enters at its posterior end, flows forward and out at the anterior end into a blood vessel that sends smaller vessels up through the gill-arches to the dorsal side.

In the amphibia the heart is a tube, so twisted on itself that the original posterior end is carried forward to the anterior end, and this part, the auricle, is divided lengthwise by a partition into a right and a left side. In the reptiles the ventricle is also partially separated into two chambers, completely so in the crocodiles. In birds and mammals the auricular and ventricular septa are complete in the adult, and the ventral aorta that carries the blood forward from the heart is completely divided into two vessels, one of which now carries blood to the lungs. When we examine the development of the heart of a mammal, or of a bird, we find something like a parallel series of stages, apparently resembling conditions found in the different groups just described. The heart is, at first, a straight tube, it then bends on itself, and a constriction separates the auricular part from the ventricular, and another the ventricular from the ventral aorta. Vertical longitudinal partitions then arise, one of which separates the auricle into two parts, and another the ventricle into two parts, and a third divides the primitive aorta into two parts. In the early stages all the blood passes from the single ventral aorta through the gill-arches to the dorsal side, and it is only after the appearance of the lung-system that the gillsystem is largely obliterated.

We find here, then, a sort of parallel, provided we do not inquire too particularly in to details. This comparison may be justified, at least so far that the circulation is at first through the arches and is later partially replaced by the double circulation, the systemic and the pulmonary.

A few other cases may also be added. The proverbial absence of teeth in birds applies only to the adult condition, for, as first shown by Geoffroy Saint-Hilaire, four thickenings, 
or ridges, develop in the mouth of the embryo; two in the upper, two in the lower, jaw. These ridges appear to correspond to those of reptiles and mammals, from which the teeth develop. It may be said, therefore, that the rudiments of teeth appear in the embryo of the bird. This might be interpreted to mean that the embryo repeats the ancestral reptilian stage, or, perhaps, the ancestral avian stage that had teeth in the beak; but since only the beginnings of teeth appear, and not the fully formed structures, this interpretation would clearly overshoot the mark.

The embryo of the baleen whale has teeth that clo not break through the gums and are later absorbed. Since the ancestors of this whale probably had teeth, as have other whales at the present time, the appearance of teeth in the embryo has been interpreted as a repetition of the original condition. Some of the ant-eaters are also toothless, but teeth appear in the embryo and are lost later. In the ruminants that lack teeth in the front part of the upper jaw. $c . g$. the cow and the sheep, teeth develop in the embryo which are subsequently lost.

One interpretation of these facts is that the ancestral adult conclition is repeated by the embryo, but as I have pointed out above in the cases of the teeth in whales, since the teeth do not reach the adult form, and do not even break through the grums in some forms, it is obviously stretching a point to claim that an adult condition is repeated. Moreover, in the case of the birds only the dental ridges appear, and it is manifestly absurd to claim in this case that the ancestral adult condition of the reptiles is repeated.

That a supposed ancestral stage may be entirely lost in the embryo of higher forms is beautifully shown in the derelopment of some of the snakes. The snakes are probably derived from lizardlike ancestors, which had four legs, yet in the development the rudiments of legs do not appear, and this is the more surprising since a few snakes have small 
rudimentary legs. In these, of course, the rudiments of legs must appear in the cmbryo, but in the legless forms even the beginnings of the legs have been lost, or at any rate very nearly so.

Outside the group of vertebrates there are also many cases that have been interpreted as embryonic repetitions of ancestral stages, but a brief examination will suffice to show that many of these cases are doubtful, and others little less than fanciful. A few illustrations will serve our purpose. The most interesting case is that given by the history of the nauplius theory.

The free-living larva of the lower crustaceans - waterflees, barnacles, copepods, ostracods - emerges from the egcr as a small, flattened oval form with three pairs of appendages. This larva, known as the nauplius, occurs also in some of the higher crustaceans, not often, it is true, as a free form, as in penæus, but as an embryonic stage. The occurrence of this six-legged form throughout the group was interpreted by the propounders of the nauplius theory as evidence sufficient to establish the view that it represented the ancestor of the whole group of Crustacea, which ancestor is, therefore, repeated as an cmbryonic form. This hypothesis was accepted by a large number of eminent embryologists. The history of the collapse of the theory is instructive.

It had also been found in one of the groups of higher crustaceans, the decapods, containing the crayfish, lobster, and crabs, that another characteristic larval form was repeated in many cases. This larva is known as the zoëa. It has a body made up of a fused head and thorax carrying seven pairs of appendages and of a segmented abdomen of six segments. The same kind of evidence that justified the formulation of the nauplius theory would lead us to infer that the zoëa is the ancestor of the decapods. The later development of the zoëa shows, however, that it cannot be such an ancestral form, for, in order to reach the 
full number of segments characteristic of the decapods, new segments are intercalated between the cephalothorax and abdomen. In fact, in many zoëas this intercalated region is already in existence in a rudimentary condition, and small appendages may even be present. A study of the comparative anatomy of the crustaceans leaves no grounds for supposing that the decapods with their twenty-one segments have been evolved from a thirteen-segmented form like the zoëa by the intercalation of eight segments in the middle of the body. It follows, if this be admitted, and it is generally admitted now, that the zoëa does not represent an original ancestral form at all, but a highly modified new form, as new, perhaps, as the group of clecapods itself. We are forced to conclude, then, that the presence of a larval form throughout an entire group caunot be accepted as evidence that it represents an ancestral stage. We can account for the presence of the zoëa, however, by making a single supposition, namely, that the ancestor from which the group of decapod has evolved had a larva like the zoëa, and that this larval form has been handed down to all of the descendants.

The fate of the zoëa theory cast a shadow over the nauplius theory, since the two rested on the same sort of evidence. The outeome was, in fact, that the nauplins theory was also abandoned, and this was seen to be the more necessary, since a study of the internal anatomy of the lowest group of crustaceans, the phyllopods, showed that they have probably come directly from many segmented, annelidian ancestors. The presence of the nauplius is now generally accounted for by supposing that it was a larval form of the ancestor from which the group of crustaceans arose.

The most extreme, and in many ways the most uncritical, application of the recapitulation theory was that made by Hackel, more especially his attempt to reduce all the higher animals to an ancestral double-walled sac with an opening 
at one end, - the gastrea. He dignified the recapitulation theory with an appellation of his own, "The Biogenetic Law." Haeckel's fanciful and extreme application of the older recapitulation theory has probably done more to bring the theory into disrepute amongst embryologists than the criticisms of the opponents of the theory.

In one of the recognized masterpieces of embryological literature, His's "Unsere Körperform," we find the strongest protest that has yet been made against the Haeckelian pretension that the phylogenetic history is the "cause" of the ontogenetic series. His writes: "In the entire series of forms which a developing organisni runs through, each form is the necessary antecedent step of the following. If the embryo is to reach the complicated end-forms, it must pass, step by step, through the simpler ones. Each step of the series is the physiological consequence of the preceding stage and the necessary condition for the following. Jumps, or short cuts, of the developmental process, are unknown in the physiological process of development. If embryonic forms are the inevitable precedents of the mature forms, because the more complicated forms must pass through the simpler ones, we can understand the fact that paleontological forms are so often like the embryonic forms of to-day. The paleontological forms are embryonal, because they have remained at the lower stage of development, and the present embryos must pass also through lower stages in order to reach the higher. But it is by no means necessary for the later, higher forms to pass through embryonal forms because their ancestors have once existed in this condition. To take a special case, suppose in the course of generations a species has increased its length of life gradually from one, two, three years to eighty years. The last animal would have had ancestors that lived for one year, two years, three years, etc., up to eighty years. But who would claim that because the final eighty-year species must pass necessarily through one, 
two, three years, etc., that it does so because its ancestors lived one year, two years, three years, etc.? The descent theory is correct so far as it maintains that older, simpler forms have been the forefathers of later complicated forms. In this case the resemblance of the older, simpler forms to the embryos of later forms is explained without assuming any law of inheritance whatsoever. The same resemblance between the older and simpler adult forms, and the present embryonic forms would even remain intelligible were there no relation at all between them."

Interesting and important as is this idea of His, it will not, I think, be considered by most embryologists as giving an adequate explanation of many facts that we now possess. It expresses, no doubt, a part of the truth but not the whole truth.

We come now to a consideration of certain recently ascertained facts that put, as I shall try to show, the whole question of embryonic repetition in a new light.

A minute and accurate study of the early stages of division or clearage of the egg of annelids has shown a remarkable agreement throughout the group. The work of E. 13. Wilson on nereis, and on a number of other forms, as well as the subsequent work of Mead, Child, and Treadwell on other annelids, has shown resemblances in a large number of details, involving some very complicated processes. ${ }^{1}$

Not only is the same method of cleavage found in most annelids, but the same identical form of division is also present in many of the mollusks, as shown especially by the work of Conklin, Lillie, and Holmes. This resemblance has been cliscussed at some length by those who have worked out these results in the two groups. The general conclusion reached by them is that the only possible interpretation of the

\footnotetext{
1 On the other hand it should not pass unnoticed that Eisigh as shown in one form (in which, however, the egrgs are under special conditions being closely packed together) that the usual type of cleavage is altered.
} 
phenomenon is that some sort of genetic connection must exist between the different forms; and while not explicitly stated, yet there is not much doubt that some at least of these authors have had in mind the view that the annelids and mollusks are descended from common ancestors whose eggs segmented as do those of most of the mollusks and annelids of the present day. This conclusion is, I believe, of more far-reaching importance than has been supposed, and may furnish the key that will unlock the whole question of the resemblance of embryos to supposed ancestral forms. It is a most fortunate circumstance that in the case of this cell lineage the facts are of such a kind as to preclude the possibility that the stages in common could ever have been ancestral adult stages. If this be granted then only two interpretations are possible: the results are due either to a coincidence, or to a common embryonic form that is repeated in the embryo of many of the descendants. That the similarity is not due to a coincidence is made probable from the number and the complexities of the cleavage stages.

I believe that we can extend this same interpretation to all other cases of embryonic resemblance. It will explain the occurrence of gill-slits in the embryo of the bird, and the presence of a notochord in the higher forms in exactly the same way as the cleavage stages are explained. But how, it may be asked, can we explain the apparent resemblance between the embryo of the higher form and the adult of lower groups. The answer is that this resemblance is deceptive, and in so far as there is a resemblance it depends on the resemblance of the adult of the lower form to its own embryonic stages with which we can really make a comparison. The gill-slits of the embryo of the chick are to be compared, not with those of the adult fish, but with those of the embryo of the fish. It is a significant fact, in this connection, that the gill-slits appear as early in the embryo of the fish as they do in the bird! The notochord of the embryo bird is 
comparable with that of the embryo of amphioxus, and not with the persistent notochord in the adult amphioxus. Here also it is of the first importance to find that the notochord appears both in the embryo bird and in amphioxus at the very beginning of the development. The embryo bircl is not fishlike except in so far as there are certain organs in the embryo fish that are retained in the adult form. The embryo bird bears the same relation to the embryo fish that the early segmentation stages of the mollusk bear to the early segmentation stages of the annelid. There are certain obvious resemblances between this view and that of Von Baer, but there are also some fundamental differences between the two conceptions.

Von Baer thought that within each group the embryonic development is the same up to a certain point. He supposed that the characters of the group are the first to appear, then those of the order, class, family, genus, and, finally, of the species. He supposed that two similar species would follow the same method of development until the very last stage was reached, when each would then add the final touches that give the individual its specific character. We may call this the theory of embryonic parallelism. Here there is an important difference between my view and that of Von Baer, for I should not expect to fincl the two embryos of any two species identical at any stage of their development, but at most there might exist a close resemblance between them.

Von Baer's statement appears to be erroneous from a moclern point of view in the following respects. We know that in certain large groups some forms develop in a very different way from that followed by other members of the group, as shown by the cephalopods, for instance, in the group of mollusks. Again, it is entirely arbitrary to assume that the groupcharacters are the first to appear, and then successively those of the order, family, cremus, species. Finally, as has been said above, we do not find the early embryos of a 
group identical; for with a sufficient knowledge of the development it is always possible to distinguish between the embryos of different species, as well as between the adults, only it is more difficult to do so, because the embryonic forms are simpler. The most fundamental difference between the view of Von Baer and modern views is due to our acceptation of the theory of evolution which seems to make it possible to get a deeper insight into the meaning of the repetition, that carries us far ahead of Von Baer's position. For with the acceptance of this doctrine we have an interpretation of how it is possible for the embryonic stages of most members of a group to have the same form, although they are not identical. There has been a continuous, although divergent, stream of living material, carrying along with it the substance out of which the similar embryonic forms are made. As the stream of embryonic material clivided into different paths it has also changed many of the details, sometimes even all; but nevertheless it has often retained the same general method of development that is associated with its particular composition. We find the likeness, in the sense of similarity of plan, accounted for by the inheritance of the same sort of substance; the differences in the devclopment must be accounted for in some other way.

Among modern writers Hurst alone has advanced a view that is similar in several respects to that which I have here defended. It may be well to give his statement, since it brings out certain points of resemblance with, as well as certain differences from, my own view. ${ }^{1}$ He says: "Direct observation has shown that, when an animal species cartics (i.c. bccomcs unlike what it was before) in adult structure, those stages in the development which are nearest the adult undergo a similar, but usually smaller, change. This is shown in domestic species by the observations of Darwin, and the

${ }^{1}$ Hurst, C. H., “ Biological Theories, III," “The Recapitulation Theory," Natural Science, Vol. ii., I $\$ 93$. 
result is in exact harmony with the well-known law of Von Baer, which refers to natural species, both nearly related and widely dissimilar. Von Baer's observations as well as Darwin's, and as well as those of every student who has ever compared the embryos of two vertebrate species, may be summarized as follows:-

"Animals which, though related, are very similar in the adult state, resemble each other more closely in early stages of clevelopment, often, indeed, so closely as to be indistinguishable in those early stages. As development proceeds in such species, the differences between the two embryos compared become more and more pronounced." On this point, which is an essential one, I cannot agree with Hurst; for I do not think that the facts show that the early stages of two related forms are necessarily more and more alike the farther back we go. The resemblance that is sometimes so striking in the earlier stages is due to the fewer points there are for comparison, and to the less development of the parts then present. Hurst continues: "If similar comparisons could be instituted between the ancestral species and its much modified descendants, there is no reason for doubting that a similar result would be reached. This, indeed, has been done in the case of some breeds of pigeons, which we have excellent reasons for believing to be descended from Columba liviar. True, $C$. livia is not a very remote ancestor, but I do not think that will vitiate the argument. Let me quote Darwin verbatim: "As we have conclusive evidence that the breeds of the pigeon are descended from a single wild species, I have compared the young within twelve hours after being hatched; I have carefully measured the proportions (but will not here give the details) of the beak, width of mouth, length of nostril, and of eyelid, size of feet, and length of leg in the wild, parent species, in pouters, fantails, runts, barbs, dragrons, carriers, and tumblers. Now some of these birds when mature differ in so extraordinary a manner in the 
length and form of the beak, and in other characters, that they would certainly have been ranked as distinct genera if found in a state of nature. But when the nestling birds of these several breeds were placed in a row, though most of them could just be distinguished, the proportional differences in the above specified points were incomparably less than in the full-grown birds. Some characteristic points of difference - for instance, that of the width of the mouth - could hardly be detected in the young. But there was one remarkable exception to this rule, for the young of the short-faced tumbler differed from the young of the wild-rock pigeon, and of the other breeds in almost exactly the same proportions as in the adult state.'"

Hurst concludes that: "The more the adult structure comes to be unlike the adult structure of the ancestors, the more do the late stages of development undergo a modification of the same kind. This is not mere dogma, but it is a simple paraphrase of Von Baer's law. It is proved true not only by the observations of Von Baer and of Darwin, already referred to, but by the direct observation of every one who takes the trouble to compare the embryos of any two vertebrates, provided only he will be content to see what actually lies before him and not the phantasms which the recapitulation theory may have printed on his imagination."

The growth of the antlers of stags is cited by Hurst in order to illustrate that what has been interpreted as a recapitulation may have a different interpretation. "Each stag develops a new pair of antlers in each successive year, and each pair of antlers is larger than the pair produced in the previous year. This yearly increase in the size of the antlers has been put forward as an example of an ontogenetic record of past evolution. I, however, deny that it is such a record."

"The series of ancestors may have possessed larger antlers in each generation than in the generation before it. It is not 
an occasional accidental parallelism between the ontogeny and the phylogeny which I deny, but the causal relation between the two. Had the ancestors had larger antlers than the existing ones, there is no justification for the assumption that existing stags would acquire antlers of which each pair, in later years, would be smaller than those of the previous year."

Hurst concludes: "There are many breeds of hornless sheep, but they do not bear large horns in early years and then shed them. If a rudiment ever appears in the embryo of such sheep, its growth is very early arrested." The case of the appendix in man might have been cited here as a case in point. It is supposed to have been larger in the ancestors of man, but we do not find it appearing full size in the embryo and later becoming rudimentary. The preceding statements will show that, while Hurst's view is similar in some respects to my own, yet it cliffers in one fundamental respect from it, and in this regard he approaches more nearly to the theory of Von Baer.

Hertwig has recently raised some new points of issue in regard to the recapitulation theory, and since he may appear to have penetrated farther than most other embryologists of the present time, it will be necessary to examine his view somewhat carefully. He speaks of the germ-cell (egg, or spermatozoön) as a species-cell, because it contains, in its finer organization, the essential features of the species to which it belongs. There are as many of these kinds of cells as there are different kinds of animals and plants. Since the bodies of the higher animals have developed from these species-cells, so the latter must have passed in their phylogeny through a corresponding development from a simple to a more and more complex cell-structure. "Our doctrine is, that the species-cell, even as the adult, many-celled representative of the species, has passed through a progressive, and, indeed, in general a corresponding development in the course 
of phylogeny. This view appears to stand in contradiction to the biogenetic law. According to the formula that Haeckel has maintained, the germ development is an epitome of the genealogy; or the ontogeny is a recapitulation of the phylogeny; or, more fully, the series of forms through which the individual organism passes during its development from the egg-cell to the finished condition is a short, compressed repetition of the longer series of forms which the forefathers of the same organism, or the stem-form of the species, has passed through, from the earliest appearance of organisms to the present time." "Haeckel admits that the parallel may be obliterated, since much may be absent in the ontogeny that formerly existed in the phylogeny. If the ontogeny were complete, we could trace the whole ancestry." Hertwig states further, that "The theory of biogenesis ${ }^{1}$ makes it necessary to change Haeckel's expression of the biogenetic law, so that a contradiction contained in it may be removed. We must drop the expression 'repetition of the form of extinct forefathers,' and put in its place the repetition of forms which are necessary for organic development, and lead from the simple to the complex. This conception may be illustrated by the egg-cell."

Since each organism begins its life as an egg we must not suppose that the primitive conditions of the time, when only single-celled amœbas existed on our planet, are repeated. The egg-cell of a living mammal is not, according to Hertwig's hypothesis, an indifferent structure without much specialization like an amœba, but is an extraordinarily complex end-product of a long historical process, which the organized substance has passed through. If the egg of a mammal is different from that of a reptile, or of an amphibian,

1 This term, by which Hertwig designates a particular view of his own, has been already preoccupied in a much wider sense by Huxley to mean that all life comes from preëxisting life. Hertwig means by the theory of biogenesis that as the egg develops there is a constant interchange between itself and its surroundings. 
because in its organization it contains the basis of a mammal, just so much more must it be different from the hypothetical one-celled amoba, which has no other characteristics than those that go to make up an amoba. Expressed more generally, the developmental process in the many-celled organisms begins, not where it began in primitive times, but as the representation of the highest point which the organization has at present reached. The development commences with the egg, because it is the elemental and fundamental form in which organic life is represented in connection with the reproductive process, and also because it contains in itself the properties of the species in its primordia.

"The egg-cell of the present time, and its one-celled predecessor in the phylogenetic history, the amoba, are only comparable in so far as they fall under the common definition of the cell, but beyond this they are extraordinarily different from each other."

"The phyletic series must be divided into two different kinds of processes : - First. The evolution of the species-cell, which is a steady advance from a simple to a complex organization. Second. The periodically repeated development of the manycelled individual out of the single cell, representative of the species (or the individual ontogeny), which in general follows the same rules as the preceding ontogeny, but is each time somewhat nodified according to the amount to which the species-cell has itself been changed in the phylogeny. Similar restricting and explanatory additions to the biogenetic law, like those stated here for the one-celled stage, must be made in other directions. Undoubtedly there exists in a certain sense a parallel between the phylogenetic, and the ontogenetic, development.

"On the basis of the general developmental hypothesis on which we stand, all forms which in the chain of ancestors were end-products of the individual development are now passed through by their descendants as embryonic stages, and 
so in a certain degree are recapitulated. We also admit that the embryonic forms of higher animals have many points of comparison with the mature forms of related groups standing lower in the system.

"Nevertheless, a deeper insight into the conditions relating to these resemblances shows that there are very important differences that should not be overlooked. Three points need to be mentioned: I. The cell-material which in the ancestral chain gives the basis for each ontogenetic process is each time a different material as far as concerns its finer organization and primordia. Indeed, the differences become greater the farther apart the links of the original chain become. This thought may be formulated in another way: The same ontogenetic stages that repeat themselves periodically in the course of the phylogeny always contain at bottom a somewhat different cell-material. From this the second rule follows as a consequence. 2. Between the mature end-form of an ancestor and the corresponding embryonic form of a widely remote descendant (let us say between the phylogenetic gastrea and the embryonic gastrula stage of a living mammal, according to the terminology of Haeckel) there exists an important difference, namely, that the latter is supplied with numerous primordia which are absent in the other, and which force it to proceed to the realization of its developmental process. The gastrula, therefore, as the bearer of important latent forces, is an entirely different thing from the gastræa, which has already reached the goal of its development. 3. In the third place, at each stage of the ontogeny outer and inner factors are at work, in fact even more intensely than in the fully formed organism. Each smallest change that acts anew in this way at the beginning of the ontogeny can start an impulse leading to more extensive changes in later stages. Thus the presence of yolk and its method of distribution in the egg alone suffice to bring about important changes in the cleavage, and in the formation of the germ-layers, the 
blastula, and gastrula stages," ete. "Moreover, the embryo may adapt itself to special conditions of embryonic life, and produce organs of an ephemeral nature like the amnion, chorion, and placenta."

"A comparison of ontogenetic with antecedent phylogenetic stages must always keep in view the fact that the action of external and internal factors has brought about considerable changes in the ontogenetic system, and, incleed, in a generally advaneing direction, so that in reality a later condition can never correspond to a preceding one."

Hertwig sums up his conclusion in the statement that ontogenetic stages give us, therefore, a greatly changed picture of the phylogenetic series of adult ancestors. "The two correspond not according to their actual contents but only as to their form." Hertwig also repeats His's idea, that the reason that certain kinds of form repeat themselves in the development of animals with a great constancy depends principally on this, that they supply the necessary conditions under which alone the following higher stage of the ontogeny can be formed. The development, for instanee, begins with the division of the egg, because this is the only way that a one-celled condition can give rise to a many-celled form. Agrain, the organs can be formed only when groups of cells have made a closer union with one another. Thus the gastrula must begin with the antecedent blastula, ete. Definite forms are, despite all modifying influences, held to firmly, beeause by their presence the complicated end-stages can be reached in the simplest and most suitable way.

Thus Hertwig adopts here a little from one doctrine and there a little from another, and between his attempt to reinstate the old biogenetie law of Haeckel, and to adopt a more modern point of view, he brings together a rather curious collection of statements which are not any too well coördinated. Take, for example, his description of the relation between Haeckel's gastraea and the embryonic gastrula stage. The latter he 
maintains is a repetition of the other, but only in form, not in actual contents. And in another connection we are told that the cause of this repetition is that the gastrula is the simplest way in which the later stages can be reached, and, therefore, it has been retained. It seems to me that Hertwig has undertaken an unnecessary and impossible task when he attempts to adjust the old recapitulation theory to more modern standards. His statement that the egg is entirely different from its amœba prototype is, of course, only the view generally held by all embryologists. His mystical statement that the embryonic form repats the ancestral adult stage in its form, but not in its contunts, will scarcely recommend itself as a model of clear thinking. Can we be asked to believe for instance that a young chick repeats the ancestral adult fish form but not the contents of the fish ?

In conclusion, then, it seems to me that the idca that adult ancestral stages haic been pushed back into the cmbryo, and that the cmbryo recapitulates in part these ancestral adult stages is in principle false. The resemblance between the embryos of higher forms and the adults of lower forms is due, as I have tried to show, to the presence in the embryos of the lower groups of certain organs that remain in the adult forms of this group. It is only the embryonic stages of the two groups that we are justified in comparing; and their resemblances are explained on the assumption that there has been an ancestral adult form having these embryonic stages in its development and these stages have been handed down to the divergent lines of its descendants.

Since we have come to associate with the name of the recapitulation theory the idea of the recurrence of an ancestral adult form, it may be better to find a substitute for this term. I suggest, therefore, for the view, that the embryos of the higher group repeat the modified form of the embryos of the lower groups, the term, the theory of embryonic repetition, or, more briefly, the repetition theory. 


\section{Conclusions}

In the light of the preceding discussion concerning the evidence in favor of the transmutation theory, we may now proceed to sum up our general conclusions, and at the same time discuss some further possibilities in regard to the descent theory.

The most widely accepted view in regard to the theory of organic evolution is that which looks upon the resemblances between the members of a group as due to their common descent from one original species that has broken up, as it were, into a number of new forms. Strictly applied, this means that all the vertebrates have come from one original species, all the mollusks from another, the echinoderms from a third, etc. Even farther back there may have been a common ancestral species for any two of the large groups, as, for example, the annelids and the mollusks; and if the relationship of all the many-celled forms be looked upon as probable, then they too have originated from one ancestral species.

Many zoologists appear to hesitate to apply strictly this fundamental idea contained in the transmutation theory, because, perhaps, they feel that it does not fit in with their general experience of living forms. Yet there can be no doubt that it is the primary conception of the transmutation theory. This is, however, not the whole question, for we must further consider the number of individuals of a species that are involved.

In some species there are smaller groups of inclividuals that are more like one another than like other inclividuals of the same species. Such groups are called varieties, and are often associated with certain localities, or with a special environment. In the latter case they are called local varieties. Some of these appear to breed true, not only when kept under the same conditions, but even when transferred 
to a new environment. Others change with the environment. It is not improbable that the varieties are of a different kind in these two cases, as shown by their different behavior when put under new and different surroundings. The variety that owes its peculiarities, not to the immediate environment, but to some internal condition independent of the surroundings, is recognized by some biologists as a smaller species. Such species appear to be commoner in plants than in animals, although it is possible that this only means that more cases have been found by the botanists, owing to the greater ease with which plants can be handled. These smaller species, in contradistinction to the ordinary Linnzean species, differ from the latter in the smaller amount of differences between the groups, and probably also in that they freely interbreed, and leave fertile descendants; but whether this is only on account of the smaller differences between them than between larger species, or because of some more fundamental difference in the kind of variation that gives rise to these two kinds of groups, we do not know.

These smaller species, or constant varieties, as we may call them, may be looked upon as incipient Linnean species, which, by further variations of the same, or of other sorts, may end by giving rise to true species. A genus composed of several species might be formed in this way, and then, if each species again broke up into a number of new groups, each such group would now be recognized as a genus, and the group of genera would form a family, etc. The process continuing, a whole class, or order, or even phylum, might be the result of this process that began in a single species.

But we must look still farther, and inquire whether the start was made from a single individual, that began to vary, or from a number of individuals, or even from all the individuals, of a species. If we suppose the result to depend on some external cause that affects all the individuals of a species alike, then it might appear that the species, or at 
least as many individuals of a species as are affected, will give the starting-point for the new group. But if the new variation arises not directly as a response to some change in the surroundings, then it might appear in one or in a few individuals at a time. Let us consider what the results might be under these two heads.

If amongst the descendants of a single individual a new form or a number of new forms were to arise, then, if they represented only a variety, they would cross with the other forms like the parent species; and, under these conditions, it is generally assumed that the new variety would be swamped. If, however, the new forms have the value of new species, then, $c x$ hypothcsc, they are no longer fertile with the original forms, and might perpetuate themselves by self-fertilization, as would be possible in some of the higher plants, and in those animals that are bisexual. But as a rule even bisexual forms are not self-fertilized, and, therefore, unless a number of offspring arose from the same form the chance of propagation would be small.

If, however, a number of new forms appeared at the same time and left a number of descendants, then the probability that the new group might perpetuate itself is greater, and the chance that such a group would arise is in proportion to the number of inclividuals that varied in the same direction simultaneously. In this case the new species has not come from a single individual or even from a pair of individuals, but from a number of individuals that have varied more or less in the same direction.

This point of view puts the descent theory in a somewhat unforeseen light, for we cannot assume in such a case that the similarities of the members of eren the same species are due to direct descent from an original ancestor, because there are supposed to have been a number of ancestors that have all changed in the same direction. The question is further complicated by the fact that the new individuals begin to 
interbreed, so that their descendants come to have, after a time, the common blood, so to speak, of all the new forms. If with each union there is a blending of the substances of the individuals, there will result in the end a common substance representing the commingled racial germ-plasm.

A new starting-point is then reached, and new species may continue to be formed out of this homogeneous material. Thus, in a sense, we have reached a position which, although it appears at first quite different from the ordinary view, yet, after all, gives us the same standpoint as that assumed by the transmutation theory; for, while the latter assumes that the resemblances of the members of a group are due to descent from the same original form, and often by implication from a single individual, we have here reached the conclusion that it is only a common, commingled germ-plasm that is the common inheritance.

When we examine almost any group of living animals or plants, whether they are low or high in organization, we find that it is composed of a great many different species, and so far as geology gives any answer, we find that this must have been true in the past also. Why, then, do we suppose that all the members of the higher groups have come from a single original species or variety? Why may not all, or many, of the similar species of the lower group have changed into the species of the higher group, - species for species? If this happened, the resemblance of the new species of the group could be accounted for on the supposition that their ancestors were also like one another. The likeness would not be due, then, to a common descent, and it would be false to attempt to explain their likeness as.due to a common inheritance. But before going farther, it may be well to inquire to what the resemblances of the individuals of the original species were due; for, if they have come from an older group that has given rise to divergent lines of descent, 
then we are only remoring the explanation one step farther back. If this original group has come from numerous species of a still older group, and this, in turn, from an older one still, then we must go back to the first forms of life that appeared on the globe, and suppose that the individuals of these primitive forms are the originals of the species that we find living to-day. For instance, it is thinkable that each species of vertebrate arose from a single group of the earliest forms of life that appeared on the surface of the earth. If this were the case, there must have been as many different kinds of species of the original group as there are species alive at the present time, and throughout all the past. This view finds no support from our knowledge of fossil remains, and, although it may be admitted that this knowledge is very incomplete, yet, if the process of evolution had taken place as sketched out above, we should expect, at least, to have found some traces of it amongst fossil forms. Since this question is an historical one, we can, at best, only expect to decicle which of all the possible suggestions is the more probable.

We conclude, then, that it is more probable that the vertebrates, the mollusks, the insects, the crustaceans, the annelids, the coelenterates, and the sponges, ete, have come each from a single original species. Their resemblances are due to a common inheritance from a common ancestral species. Even if it be probable that at the time when the group of vertebrates arose from a single species, there were in existence other closely related species, yet we must suppose, if we adhere to our point of view, that these other related species have had nothing to do with the group of vertebrates, but that they have died out. Noreover, we must suppose that each order, each class of vertebrate, has come from a single origimal species; each family has had a similar origin, as well as each semus, but, of course, at different periods of time. I et us not shrink from carrying this principle to its most extreme point, for, unless the principle is absolutely true, then our 
much boasted explanation of the resemblances of forms in the same group will be thrown into hopeless confusion.

Let us ask another question in this connection. If a single species gave rise to a group of new species that represented the first vertebrates, they would have formed the first genus; and if the descendants of these diverged again so that new genera were formed, then a group which we should call a family would have been formed.

As the divergence went on, an order would be developed, and then a class, and then a phylum. The common characters possessed by the members of this phylum would have been present in the original species that began to diverge. Hence, we find the definition of the phylum containing only those points that are the features possessed by all of the descendants, and in the same way we should try to construct the definition of each of the subordinate groups. This is the ideal of the principle of classification based on the theory of descent with divergence. If we admit the possibility of the other view that I have mentioned above, or of any other of the numerous possibilities that will readily suggest themselves, then we must be prepared to give up some of the most attractive features of the explanation of resemblance as due to descent.

That all biologists believe strictly in divergent descent, to the exclusion of any other processes, is not the case. And, as I have said before, since we are dealing with an historical question, it would be very unwise, in our present ignorance on many points, to pretend that we have any direct proof of the explanation that we find generally given to account for the resemblances of the species of a group to each other. At most we can claim that it is the simplest point of view, and that most biologists believe it to be also the most probable. It has been suggested that, in some cases, the new forms that arise from two or more species rum a parallel course. If the original forms from which they came were 
very much alike, it would soon be impossible to say what the parentage of a particular form was; that is, to which of the two original forms it belonged. It has also been suggested that even a convergence has at times taken place, so that the descendants of different species have become more alike than the original forms, at least in some onc or more respects. This last limitation is the saving clause, for species differ in so many points that, even when they converge in a few, it is unlikely that they will do so in all, and, therefore, the deception may be discovered by the acute observer. One famous paleontologist has gone so far even as to suppose that a species may change its generic characters, so that it goes over bodily into a new genus without losing its specific characters. If such things do occur, then our classifications may well be the laughing-stock of Nature. 


\section{CHAPTER IV}

\section{DARWIN'S THEORIES OF ARTIFICIAL AND OF NATURAL SELECTION}

\section{The Principle of Selection}

DARIVIN's theory of natural selection is preëminently a theory of adaptation. It appears, in fact, better suited to explain this phenomenon than that of the "origin of species." Darwin prepared his reader for the ideas contained in the theory of natural selection by a brief consideration of the results of artificial selection; and since the key to the situation is, I believe, to be found in just this supposed resemblance, we cannot do better than examine the theories in the order followed by Darwin himself.

One of the means by which the artificial races of animals and plants have been formed by man is selection. The breeder picks out individuals having a certain peculiarity, and allows them to breed together. He hopes to find among the offspring, not only individuals like the parent forms, but also some that have the special peculiarity even more strongly developed. If such are found, they are isolated and allowed to breed, and in the next generation it is hoped to find one or more new individuals that show still more developed the special character that is sought. This process, repeated through a number of generations, is supposed to have led to the formation of many of our various forms of domesticated animals and plants.

This heaping up as a result of the union of similar individuals cannot for a moment be supposed to be the outcome of the addition of the two variations to each other. Such an 
idea is counter to all the most familiar facts of inheritance. For instance, when two similar forms unite, we do not find that the young show all the characters of the mother plus all those of the father, i.e. each peculiarity that is the same in both, increased twofold. On the contrary, the young are in the vast majority of cases not essentially different from either parent.

A more thorough examination of the facts shows that the problem is by no means so simple as the preceding general statement might lead one to suppose, for our experience shows that it is not always possible to increase all variations by selcction, and, furthermore, there is very soon found a limit, even in favorable cases, to the extent to which the process can be carried. The most important point appears to be the nature of the variations themselves which may arise from different causes, and which have different values in relation to the possibility of their continuation.

We may begin, therefore, by following Darwin in his analysis of variation, as given in the opening chapter of the "Origin of Species." He thinks that the great amount of variation shown by domesticated animals and plants is due, in the first place, to the new conditions of life to which they are exposed, and also to the lack of uniformity of these conditions. Darwin thinks, also, that there is some probability that this variability is due, in part, to an cxcess of food. "It seems clear that organic beings must be exposed during sereral generations to new conditions to cause any great amount of variation, and that when the organization has once begun to vary, it generally continues varying for many generations. No case is on record of a variable organism ceasing to vary under cultivation. Our oldest cultivated plants, such as wheat, still yield new varieties; om oldest domesticated animals are still capable of rapid improvement or modification." In this statement of barwin, full of significance, we must be careful to notice that he does not mean to imply, when he 
states that an organism that has once begun to vary continues to vary for many generations, that this continuous variation is always in the same direction, but only that new combinations, scattering in all directions, continue to appear.

The nature of the organism seemed to Darwin to be a more important factor in the origin of new variations than the external conditions, "for nearly similar variations sometimes arise under, as far as we can judge, dissimilar conditions; and, on the other hand, dissimilar variations arise under conditions which appear to be nearly uniform." The following statement is important in connection with the origin of "definite" variations. "Each of the endless variations which we see in the plumage of our fowls must have had some efficient cause; and if the same causes were to act uniformly during a long series of generations on many individuals, all probably would be modified in the same direction." Here we find an explicit statement in regard to the accumulation of variation in a given direction as the result of an external agent, but Darwin hastens to add: "Indefinite variability is a much more common result of changed conditions than definite variability, and has probably played a more important part in the formation of our domestic races. We see indefinite variability in the endless slight peculiarities which distinguish the individuals of the same species, and which cannot be accounted for by inheritance from either parent or from some more remote ancestor. Even strongly marked differences occasionally appear in the young of the same litter, and in seedlings from the same seed capsule. At long intervals of time, out of millions of individuals reared in the same country and fed on nearly the same food, deviations of structure so strongly pronounced as to deserve to be called monstrosities arise; but monstrosities cannot be separated by any distinct line from slighter variations."

Another cause of variation, Darwin believes, is in the in- 
herited effect of "habit and of the use and disuse of parts," or what is generally known as the Lamarckian factor of heredity. Darwin believes that changes in the body of the parent, that are the result of the use or of the disuse of a part, may be transmitted to the descendants, and cites a number of cases which he credits to this process. As we shall deal more fully with this topic in another chapter, we may treat it here quite briefly. As an example of the inheritance of dis11se, Darwin gives the following case: "I find in the domestic duck that the bones of the wing weigh less and the bones of the leg more in proportion to the whole skeleton than do the same bones in the wild duck, and this change may be safely attributed to the domestic duck flying much less and walking more than its wild parents." The great and inherited development of the udders of cows and of goats in countries where they are habitually milked, in comparison with these organs in other countries, is given as another instance of the effect of use. "Not one of our domestic animals can be named that in some country has not drooping ears, and the view has been suggested that the drooping is due to the disuse of the muscles of the ears from the animals being seldom much alarmed."

It need scarcely be pointed out here, that, in the first case given, those ducks would have been most likely to remain in confinement that had less well-developed wings, and hence at the start artificial selection may have served to bring about the result. The great development of the udders of cows and of goats is obviously connected with the greater milk-giving qualities of these animals, which may have been selected for this purpose.

Another "law" of variation recognized by Darwin is what is ealled correlated variation. For cxample, it has been found that cats which are entirely white and have blue eyes are generally deaf, and this is stated to be confined to the males. The teeth of hairless dogs are imperfect; pigeons 
with feathered feet have skin between the outer toes, and those with short beaks have small feet, and vice versa.

Another source of variation is that of reversion, or the reappearance in the offspring of characters once possessed by the ancestors. Finally, Darwin thinks that a source of variation is to be found in modifications due to the influence of a previous union with another male, or, as it is generally called, telegony. As an example Darwin cites the famous case of Lord Morton's mare. “A nearly purely bred Arabian chestnut mare bore a hybrid to a quagga. She subsequently produced two colts by a black Arabian horse. These colts were partially dun-colored and were striped on the legs more plainly than the real hybrid or even than the quagga." 1 This case, however, is not above suspicion, since it is well known that stripes 'often appear on young horses, and the careful analysis made later by Ewart, as well as his other experiments on the possibility of the transmission of influences of this sort, puts the whole matter in a very dubious light.

These citations show that Darwin recognized quite a number of sources of variation, and, although he freely admits that "our ignorance of the laws of variation is profound," yet some at least of these sources of variation are very questionable. Be this as it may, it is important to emphasize that Darwin recognized two main sources of variation, - one of which is the indefinite, or fluctuating, variability that appears constantly in domesticated animals and plants, and the other, definite variability, or a change in a definite direction, that can often be traced to the direct action of the environment on the parent or on its reproductive cells. It is the former, i.c. the fluctuating variability, that, according to Darwin, has been used by the breeder to produce most of our domestic races. In regard to the other source of variation, the definite kind, we must analyze the facts more closely.

1 "Animals and Plants under Domestication," Chap. IX. 
A definite change in the surroundings might bring about a definite change in the next generation, because the new condition acts either on the developing organism, or on the egg itself from which the individual develops. The distinction may be one of importance, for, if the new condition only effects the developing organism directly, then, when the influence is removed, there should be a return to the former condition; but if the egg itself is affected, so that it is fundamentally changed, then the effect might persist even if the animal were returned to its former environment. More important still is Darwin's recognition of the cumulative effect in a given direction of external influences, for a new variation, that was slight at first, might, through prolonged action, continue to become more developed without any other processes affecting the organism.

From the Darwinian point of view, however, the all-important source for the origin of new forms is the fluctuating variation, which is made use of both in the process of artificial and of natural selection. We may now proceed to inquire how this is supposed to take place.

It has been stated that, by means of artificial selection, Darwin believes the breeder has produced the greater number of domesticated animals and plants. The most important question is what sort of variations he has made use of in order to produce his result. Has he made use of the fluctuating variations, or of the definite ones? It is difficult, if not impossible, to answer this question in most cases, because the breeder does not always distinguish between the two. There can be little question, however, that he may sometimes have made use of the definite kinds, whether these are the outcome of external or of intermal influences. The question has been seriously raised only in recent years, and we are still uncertain how far we can accumulate and fix a variation that is of the fluctuating kind. In a few cases it has been found that the upper limit is soon 
reached, as shown by De Vries's experiments with clover, and it is always possible that a definite variation of the right sort may arise at any stage of the process. If this should occur, then a new standard is introduced from which, as from a new base, variations fluctuating in the desired direction may be selected.

This question, before all others, ought to be settled before we begin to speculate further as to what selection is able to accomplish.

Darwin's theory is often stated in such a general way that it would be applicable to either sort of variation; but if definite variation can go on accumulating without selection, then possibly we could account for evolution without supposing any other process to intervene. Under these circumstances all that could be claimed for selection would be the destruction of those variations incapable of living, or of competing with other forms. Hence the process of selection would have an entirely negative value.

The way in which domesticated animals and plants have originated is explained by Darwin in the following significant passage :-

"Let us now briefly consider the steps by which domestic races have been produced, either from one or from several allied species. Some effect may be attributed to the direct and definite action of the external conditions of life, and some to habit; but he would be a bold man who would account by such agencies for the differences between a drayand race-horse, a greyhound and bloodhound, a carrier and tumbler pigeon. One of the most remarkable features in our domesticated races is that we see in them adaptation, not indeed to the animal's or plant's own good, but to man's use or fancy. Some variations useful to him have probably arisen suddenly, or by one step; many botanists, for instance, believe that the fuller's-teasel, with its hooks, which cannot be rivalled by any mechanical contrivance, is only a variety 
of the wild Dipsacus; and this amount of change may have suddenly arisen in a seedling. So it has probably been with the turnspit dog; and this is known to have been the case with the ancon sheep. But when we compare the drayhorse and race-horse, the dromedary and camel, the various breeds of sheep fitted either for cultivated land or mountain pasture, with the wool of one breed good for one purpose, and that of another breed for another purpose; when we compare the many breeds of dogs, each good for man in different ways; when we compare the game-cock, so pertinacious in battle, with other breeds so little quarrelsome, with 'everlasting layers' which never desire to sit, and with the bantam so small and elegant; when we compare the host of agricultural, culinary, orchard, and flower-garden races of plants, most useful to man at different seasons and for different purposes, or so beautiful in his eyes, we must, I think, look further than to mere variability. We camnot suppose that all the breeds were suddenly produced as perfect and as useful as we now see them; indeed, in many cases, we know that this has not been their history. The key is man's power of accumulative selection: nature gives successive variations; man adds them up in certain directions useful to him. In this sense he may be said to have made for himself useful breeds."

Jarwin also gives the following striking examples, which make probable the view that domestic forms have really been made by man selecting those variations that are useful to him :-

"In regard to plants, there is another means of observing the accumulated effects of selection - namely, by comparing the diversity of flowers in the different varieties of the same species in the flower-garden; the diversity of leaves, pods, of iubers, or whatever part is valued, in the kitchen-garden, in comparison with the flowers of the same varieties; and the diversity of fruit of the same species in the orchard, in 
comparison with the leaves and flowers of the same set of varieties. See how different the leaves of the cabbage are, and how extremely alike the flowers; how unlike the flowers of the heartsease are, and how alike the leaves; how much the fruit of the different kinds of gooseberries differ in size, color, shape, and hairiness, and yet the flowers present very slight differences. It is not that the varieties which differ largely in some one point do not differ at all in other points; this is hardly ever, - I speak after careful observation,- - perhaps never, the case. The law of correlated variation, the importance of which should never be overlooked, will insure some differences; but, as a general rule, it cannot be doubted that the continued selection of slight variations, either in the leaves, the flowers, or the fruit, will produce races differing from each other chiefly in these characters."

Exception may perhaps be taken to the concluding sentence, for, interesting as the facts here recorded certainly are, it does not necessarily follow that all domestic products have arisen "by the continued selection of slight variations," however probable the conclusion may appear. Darwin also believes that a process of "unconscious selection" has given even more important "results than methodical selection." By unconscious selection is meant the outcome of "every one trying to possess and breed from best individual animals." "Thus a man who intends keeping pointers naturally tries to get as good dogs as he can, and afterwards breeds from his own best dogs, but he has no wish, or expectation of permanently altering the breed. Nevertheless we may infer that this process, continued during centuries, would improve and modify any breed. . . . There is reason to believe that the King Charles spaniel has been unconsciously modified to a large extent since the time of that monarch."

The enormous length of time required to produce new species by the selection of fluctuating variations is everywhere admitted by Darwin ; nowhere perhaps more strikingly 
than in the following statement: "If it has taken centuries or thousands of years to improve or modify most of our plants up to their present standard of usefulness to man, we can understand how it is that neither Australia, the Cape of Good Hope, nor any other region inhabited by quite uncivilized man has afforded us a single plant worth culture. It is not that these countries, so rich in species, do not by a strange chance possess the aboriginal stocks of any useful plants, but that the native plants have not been improved by continued selection up to a standard of perfection comparable with that acquired by the plants in countries anciently civilized."

In reply to this, it may be said that if the selection of fluctuating variations leads to an accumulation in the given direction, it is not apparent why it should take thousands of years to produce a new race, or require such a high degree of skill as Darwin supposes the breeder to possess.

The conditions favorable to artificial selection are, according to Darwin: I. The possession of a large number of individuals, for in this way the chance of the desired variation appearing is increased. 2. Prevention of intercrossing, such as results when the land is enclosed, so that new forms may be kept apart. 3. Changed conditions, as introducing variability. 4. The intercrossing of aboriginally distinct species. 5. The intercrossing of new breeds, "but the importance of intercrossing has been much exaggerated." 6. In plants propagation of bud variations by means of cuttings. The chapter concludes with the statement, "Over all these causes of Change, the accumulative action of Selection, whether applied methodically and quickly, or unconsciously and slowly, but more efficiently, seems to have been the predominant Power."

Variability, Darwin says, is governed by many unknown laws, and the final result is "infinitely complex." If this is so, we may at least hesitate before we accept the statement 


\section{Darwin's Artificial and Natural Selection IOI}

that selection of fluctuating variations has been the only principle that has brought about these results. This is a most important point, for, as we shall see, the central question in the theory of natural selection has come to be whether by the accumulation of fluctuating variations a new species could ever be produced. If it be admitted that the evidence from artificial selection is far from convincing, in showing that selection of fluctuating variations could have been the main source, even in the formation of new races, we need not be prejudiced in faror of such a process, when we come to examine the formation of species in nature.

There are still other questions raised in this same chapter that demand serious consideration. Darwin writes as follows :

"When we look to the hereditary varieties or races of our domestic animals and plants, and compare them with closely allied species, we generally perceive in each domestic race, as already remarked, less uniformity of character than in true species. Domestic races often have a somewhat monstrous character; by which I mean, that, although differing from each other, and from other species of the same genus, in several trifling respects, they often differ in an extreme degree in some one part, both when compared one with another, and more especially when compared with the species under nature to which they are nearest allied. With these exceptions (and with that of the perfect fertility of varieties when crossed,--a subject hereafter to be discussed), domestic races of the same species differ from each other in the same manner as do the closely allied species of the same genus in a state of nature, but the differences in most cases are less in degree. This must be acmitted as true, for the domestic races of many animals and plants have been ranked by some competent judges as the descendants of aboriginally distinct species, and by other competent judges as mere varieties. If any well-marked distinction existed between a domestic race 
and a species, this source of doubt would not so perpetually recur."

The point here raised in regard to the systematic value of the new forms is the question that first demands our attention. We must exclude all those cases in which several originai species have been blended to make a new form, because the results are too complicated to make use of at present. The domesticated races of dogs appear to have had such a multiple origin, the origin of horses is in doubt; but the domesticated pigeons, ducks, rabbits, and fowls are supposed, by Darwin, to have come each from one original wild species. The great variety of the domestic pigeons gires perhaps the most striking illustration of changes that have taken place under domestication; and Darwin lays great stress on the evidence from this source.

It scems probable in this case, (I) that all the different races of pigeons have come from one original species; (2) that the structural differences are in some respects as great as those recognized by systematists as specifically distinct; (3) that the different races breed true to their kind; (4) that the result has been reached mainly by selecting and isolating rariations that have appeared under domestication, and that probably some, at least, of these variations were fluctuating ones.

Does not this grant all that Darwin contends for? In one sense, yes; in another, no! The results appear to show that by artificial selection of some kind a group of new forms may be produced that in many respects resemble a natural family, or a genus; but if this is to be interpreted to mean that the result is the same as that by which natural groups have arisen, then I think that there are good reasons for dissenting from such a conclusion. Morcover, we must not grant too readily that the different races of pigeons have arisen by the selection of fuctuating araritions alone, for this is not established with any great degree of probability by the evidence.

In regard to the first point we find that one of the most 


\section{Darwin's Artificial and Natural Selection 103}

striking differences between species in nature is their infertility, and the infertility of their offspring when intercrossed. This is a very general rule, so far as we know. In regard to the different races of domesticated forms, the most significant fact is that, no matter how different they may be, they are perfectly fertile inter sc. In this respect, as well as in others, there are important differences between domesticated races and wild species. The further difference, that has been pointed out by a number of writers, should also not pass unnoticed, namely, that the domestic forms differ from each other in the extreme development of some one character, and not in a large number of less conspicuous characters, as is the case in wild species.

These considerations show that, interesting and suggestive as are the facts of artificial selection, they fail to demonstrate the main point for which they are used by Darwin. With the most rigorous attention to the process of artificial selection, new species comparable in all respects to wild ones have not been formed, even in those cases in which the variation has been carried farthest (where the history of the forms is most completely known).

There is another point on which emphasis should be laid. If by selecting the most extreme forms in each generation and breeding from them the standard can be raised, it might appear that we could go on indefinitely in the same direction, and produce, for instance, pigeons with legs five metres long, and with necks of corresponding length. But experience has shown that this cannot be done. As Darwin frequently remarks, the breeder is entirely helpless until the desired variation appears. It seems possible, by selecting the more extreme of the fluctuating variations in each generation, that a higher plane of variation is established, and even that more extreme forms are likely to arise for a few generations; but, even if this is the case, a limit is soon reached beyond which it is impossible to go. 
The facts of observation show, that when a new variety appears its descendants are more likely, on the average, to produce proportionately more individuals that show the same variation, and some even that may go still farther in the same direction. If these latter are chosen to be the parents of the next generation, then once more the offspring may show the same advance; but little by little the advance slows down, until before very long it may cease altogether. Unless, then, a new kind of variation appears, or a new standard of variation develops of a different kind, the result of selection of fluctuating variations has reached its limit. Our experience seems, therefore, to teach us that selection of fluctuating variations leads us to only a certain point, and then stops in this direction. We get no evidence from the facts in favor of the view that the process, if carried on for a long time, could ever produce such great changes, or the kind of changes, as those seen in wild animals and plants.

\section{Variation and Conipetition in Nature}

Darwin rests his theory on the small individual variations which occur in nature, as the following quotation shows:-

"It may be doubted whether sudden and considerable deviations of structure such as we occasionally see in our domestic productions, more especially with plants, are ever permanently propagated in a state of nature. Almost every part of every organic being is so beautifully related to its complex conditions of life that it seems as improbable that any part should have been suddenly produced perfect, as that a complex machine should have been invented by man in a perfect state. Under domestication monstrosities sometimes occur which resemble normal structures in widely different animals. Thus pigs have occasionally been born with a sort of proboscis, and if any wild species of the same genus had naturally possessed a proboscis, it might have been argued 
that this had appeared as a monstrosity; but I have as yet failed to find, after diligent search, cases of monstrosities resembling normal structures in nearly allied forms, and these alone bear on the question. If monstrous forms of this kind ever do appear in a state of nature and are capable of reproduction (which is not always the case), as they occur rarely and singly, their preservation would depend on unusually favorable circumstances. They would, also, during the first and succeeding generations cross with the ordinary form, and thus their abnormal character would almost inevitably be lost."

It is clear that Darwin does not think that the sudden and large variations that sometimes occur furnish the basis for natural selection, and the final statement in the last citation (which was added in later editions of the "Origin of Species"), to the effect that if such monstrous variations appeared as single or occasional variations they would be lost by intercrossing implies that, in general, single variations would likewise be lost unless they appeared in a sufficient number of individuals to maintain themselves against the swamping effects of intercrossing.

It is necessary to quote again, in order to show that, in some cases at least, Darwin believed selection plays little or no part in the origin and maintenance of certain peculiarities that are of no use to the species. "There is one point connected with individual differences, which is extremely perplexing: I refer to those genera which have been called protean or 'polymorphic,' in which the species present an inordinate amount of variation. With respect to many of these forms, hardly two naturalists agree, whether to rank them as species or as varieties. We may instance Rubus, Rosa, and Hieracium amongst plants, several genera of insects and of Brachiopod shells. In most polymorphic genera some of the species have fixed and definite characters. Genera which are polymorphic in one country seem to be, with 
a few exceptions, polymorphic in other countries, and likewise, judging from Brachiopod shells, at former periods of time. These facts are very perplexing, for they seem to show that this kind of variability is independent of the conditions of life. I am inclined to suspect that we see, at least in some of these polymorphic genera, variations which are of no service or disservice to the species, and which consequently have not been seized on by selection to act on and accumulate, in the same manner as man accumulates in any given direction individual differences in his domesticated productions. These individual differences generally affect what naturalists consider unimportant parts; but I could show by a long catalogue of facts, that parts which must be called important, whether viewed under a physiological or classificatory point of view, sometimes vary in the individuals of the same species. I am convinced that the most experienced naturalist would be surprised at the number of cases of variability, even in important parts of structure, which he could collect on good authority, as I have collected, during a course of years."

After pointing out that naturalists have no definite standard to determine whether a group of individuals is a variety or a species, Darwin makes the highly important admissions contained in the following paragraph: "Hence, I look at individual differences, though of small interest to the systematist, as of the highest importance for us, as being the first steps toward such slight varieties as are barely thought worth recording in works on natural history. And I look at varieties which are in any degree more distinct and permanent, as steps toward more strongly marked and permanent varieties; and at the latter, as leading to subspecies, and then to species. The passage from one stage of difference to another may, in many cases, be the simple result of the nature of the organism and of the different physical conditions to which it has long been exposed; but with respect to the more important and adaptive characters, the passage from one stage of difference 
to another may be safely attributed to the cumulative action of natural selection, hereafter to be explained, and to the effects of the increased use or disuse of parts. A wellmarked variety may therefore be called an incipient species; but whether this belief is justifiable must be judged by the weight of the various facts and considerations to be given throughout this work."

In this paragraph attention should be called especially, first, to the statement in respect to the origin of varieties, which are said to arise through individual differences. It is not clear whether these differences are supposed to have appeared first in one, or in a few individuals, or in large numbers at the same time. Again, especial note should be made of the striking admission, that the passage from one stage to another may, in many cases, be the simple result of the nature of the organism and of the physical conditions surrounding it; but with respect to the more important and adaptive differences, natural selection "may safely" be supposed to have intervened. Is it to be wondered at that Darwin's critics have sometimes accused him of playing fast and loose with the origin of varieties? And since this question is fundamental for the theory of natural selection, it is much to be regretted that Darwin leaves the matter in such a hazy condition. It may be said that, at the time when he wrote, he made the best of the evidence in regard to the origin of varieties. Be this as it may, a theory standing on no better foundations than this is not likely to be found satisfactory at the present time.

We come now to the most important chapters, the third and the fourth, of the "Origin of Species," dealing with "the struggle for existence," "natural selection," or the "survival of the fittest." Behind these fatal phrases, which have become almost household words, lurk many dangers for the unwary.

"It has been seen in the last chapter that amongst organic beings in a state of nature there is some individual variability: 
indeed I am not aware that this has ever been disputed. It is immaterial for us whether a multitude of doubtful forms be called species or subspecies or varieties; what rank, for instance, the two or three hundred doubtful forms of British plants are entitled to hold, if the existence of any well-marked varieties be admitted. But the mere existence of individual variability and of some few well-marked varieties, though necessary as the foundation for the work, helps us but little in understanding how species arise in nature. How have all those exquisite adaptions of one part of the organization to another part, and to the conditions of life, and of one organic being to another being, been perfected? We see these beautiful coadaptions most plainly in the woodpecker and the mistletoe; and only a little less plainly in the humblest parasite which clings to the hairs of a quadruped or feathers of a bird; in the structure of the beetle which dives through the water; in the plumed seed which is wafted by the gentlest breeze; in short, we see beautiful adaptions everywhere and in every part of the organic world.

"Again, it may be asked, how is it that varieties, which I have called incipient species, become ultimately converted into good and distinct species, which in most cases obviously differ from each other far more than do the varieties of the same species? How do those groups of species, which constitute what are called distinct genera, and which differ from each other more than do the species of the same genus, arise? All these results, as we shall more fully see in the next chapter, follow from the struggle for life. Owing to this struggle, variations, however slight and from whatever cause proceeding, if they be in any degree profitable to the individ. uals of a species, in their infinitely complex relations to other organic beings and to their physical conditions of life, will tend to the preservation of such individuals, and will generally be inherited by the offspring. The offspring, also, will thus have a better chance of surviving, for, of the many 
individuals of any species which are periodically born, but a small number can survive. I have called this principle, by which each slight variation, if useful, is preserved, by the term Natural Selection, in order to mark its relation to man's power of selection. But the expression often used by Mr. Herbert Spencer of the Survival of the Fittest is more accurate, and is sometimes equally convenient. We have seen that man by selection can certainly produce great results, and can adapt organic beings to his own uses, through the accumulation of slight but useful variations, given to him by the hand of Nature. But Natural Selection, as we shall hereafter see, is a power incessantly ready for action, and is as inmeasurably superior to man's feeble efforts, as the works of Nature are to those of Art."

Darwin gives the following explicit statement of the way in which he intends the term "struggle for existence" to be understood: "I should premise that I use this term in a large and metaphorical sense, including dependence of one being on another, and including (which is more important) not only the life of the individual, but success in leaving progeny. Two canine animals, in time of dearth, may be truly said to struggle with each other which shall get food and live. But a plant on the edge of a desert is said to struggle for life against the drought, though more properly it should be said to be dependent on the moisture. A plant which actually produces a thousand seeds of which only one on an average comes to maturity may be more truly said to struggle with the plants of the same and other kinds which already clothe the ground. The mistletoe is dependent on the apple, and a few other trees, but can only in a far-fetched sense be said to struggle with these trees, for if too many of these parasites grow on the same tree, it languishes and dies. But several seedling mistletoes, growing close together on the same branch, may more truly be said to struggle with each other. As the mistletoe is dis- 
seminated by birds, its existence depends on them, and it may metaphorically be said to struggle with other fruit-bearing plants, in tempting the birds to devour and thus disseminate its seeds. In these several senses, which pass into each other, I use for convenience' sake the general term 'Struggle for Existence." "

A number of writers have objected to the general and often vague way in which Darwin makes use of this phrase; but it does not seem to me that this is a serious objection, provided we are on our guard as to what the outcome will be in each case. In each instance we must consider the question on its own merits, and if it is found convenient to have a sufficiently general and non-committal term, such as the "struggle for existence," to include all cases, I see no serious objection to the use of such an expression, although it is true the outcome has been that it has become a catchword, that is used too often by those who have no knowledge of its contents.

Were it not that each animal and plant gives birth, on an average, to more than two offspring, the species would soon become exterminated by accidents, etc. We find in some of the lower animals, and in some of the higher plants, that thousands and even millions of eggs are produced by a single individual in the course of its life. A single nematode may lay sixty million eggs, and a tapeworm one thousand million. A starfish may produce about thirty-nine million eggs, a salmon may contain fifteen thousand, and a large shad as many as one hundred thousand. The queen of a termite nest is said to lay eighty thousand eggs a day.

In the higher vertebrates the number of young is considerably less, but since the young stages are passed within the body of the parent, proportionately more of them reach maturity, so that even in man the population may be doubled in twenty-five years, and in the elephant, slowest breeder of all animals, Darwin has calculated that, if it begins breeding 
when about thirty years old and goes on until ninety years, bringing forth six young in the interval, after 750 years there will be nearly nineteen million elephants alive which have descended from the first pair.

Obviously, then, if all the descendants of all the individuals of a species were to remain alive, the world would be overcrowded in a very short time, and the want of room would in itself lead to the destruction of countless individuals, if for no other reason than lack of food. We can easily carry out on a small scale an experiment that shows how the overstocking, resulting from favorable conditions, comes about, and how it checks itself. If we make a meat broth suitable for the life of a particular bacterium, and sow in the broth a very few individuals, we find in the course of several days the fluid swarming with the descendants of the original individuals. Thus it has been shown that, if we start with a few hundred bacteria, there will be five thousand after twenty-four hours, and twenty thousand, forty-eight hours later; and after four days they are beyond calculation.

Cohn found that a single bacterium produces two individuals in one hour, and four in two hours, and if they continue to multiply at this rate there will be produced at the end of three days 4,772 billions of descendants. If these are reduced to weight, they would weigh seventy-five hundred tons. Thus when the conditions are favorable, bacteria are able to increase at such an enormous rate that they could cover the surface of the earth in a very few days. The reason that they do not go on increasing at this rate is that they soon exhaust the food supply, and the rate of increase slows down, and will finally cease altogether. If the bacteria were dependent on a continuous supply of food, they would perish after the supply had been exhausted, so that the rapid rate of multiplication would serve only to bring the career of the organism to an untimely end. If the weaker individuals were to die first, the products of their dis- 
integration might serve to nourish the stronger individuals; hunger coming on again, the next weakest might die; and the same process continuing, we might imagine that the bacteria were finally reduced to a single one which would then die in turn for lack of food. Like a starving shipload of men, reduced by hunger to cannibalism, the life of some and finally of the last individual might be prolonged in the hope of rescue, but if this did not arrive, the last and perhaps the strongest individual would perish. But this is not what we find occurring in these lower organisms, for, as a rule, they gradually cease to increase when the food supply becomes lessened, and their activities slow down. Finally, when the food is gone, they pass into a resting stage, in which condition they can remain dormant for a long time, even for years. If they should again find themselves in favorable surroundings, they become active, and begin once more their round of multiplication. We cannot follow the individuals in such a culture of bacteria, but there is nothing to be seen that suggests a struggle for existence, if this idea conveys the impression of the destruction of certain individuals by competition with others. In fact, the results are in some respects exactly the reverse. Millions of individuals are present at the time when the food supply becomes exhausted, and they all pass into a protected resting stage.

The enormous rate of increase in this case finds its counterpart in higher animals when the food supply, or the absence of enemies, allows a species to multiply at its maximum rate of increase. The introduction of rabbits into Australia was followed by an enormous increase in a few years, and the introduction of the English sparrow into the United States has had a similar result. But in no country can such a process continue beyond a certain point, because, in the first place, the scarcity of food will begin to keep the birth-rate down, and in the second place, the increase in numbers may lead to an increase in the number of its enemies, or even 
incluce other forms to feed on it. Crowding will also give an opportunity for the spread of disease, which again may check the increase. Sooner or later a sort of ever shifting balance will be reached for each species, and after this, if the conditions remain the same, the number of individuals will keep approximately constant.

Darwin admits that the "causes which check the natural tendency of each species to increase are most obscure." "We know not exactly what the checks are even in a single instance." This admission may well put us on our guard against a too ready acceptation of a theory in which the whole issue turns on just this very point, namely, the nature of the checks to increase. Darwin gives the following general cases to show what some of the checks to increase are. He states that eggs and very young animals and seeds suffer more than the adults; that "the amount of food for each species of course gives the extreme limit to which each can increase; but very frequently it is not the obtaining food, but the serving as prey to other animals which determines the average numbers of a species. Thus, there seems to be little doubt that the stock of partridges, grouse, and hares on any large estate depends largely on the destruction of the vermin." "On the other hand, in some cases, as with the elephant, none are destroyed by beasts of prey; for even the tiger in India most rarely dares to attack a young elephant protected by its dam." "Climate plays an important part in determining the average number of a species, and periodical seasons of extreme cold or drought seem to be the most effective of all checks." "The action of climate seems at first sight to be quite independent of the struggle for existence; but in so far as climate acts in reducing food, it brings on the most severe struggle between the individuals, whether of the same, or of distinct species which subsist on the same kind of food."

We need not follow Darwin through his account of how complex are the relations of all animals and plants to one 
another in the struggle for existence, for, if true, it only goes to show more plainly how impossible it is to establish any safe scientific hypothesis, where the conditions are so complex and so impossible to estimate. To show that the young Scotch fir in an enclosed pasture is kept down by the browsing of the cattle, and in other parts of the world, Paraguay for instance, the number of cattle is determined by insects, and that the increase of these flies is probably habitually checked by other insects, leads to a bewilderingly complex set of conditions. We cannot do better than to quote Darwin's conclusion: "Hence, if certain insectivorous birds were to decrease in Paraguay, the parasitic insects would probably increase; and this would lessen the number of the navel-frequenting flies - then cattle and horses would become feral, and this would certainly greatly alter (as indeed I have observed in parts of South America) the vegetation : this again would largely affect the insects; and this, as we have just seen in Staffordshire, the insectivorous birds, and so onwards in ever increasing circles of complexity. Not that under nature the relations will ever be as simple as this. Battle within battle must be continually recurring with varying success; and yet in the long run the forces are so nicely balanced, that the face of nature remains for long periods of time uniform, though assuredly the merest trifle would give the victory to one organic being over another. Nevertheless, so profound is our ignorance, and so high our presumption, that we marvel when we hear of the extinction of an organic being; and as we do not see the cause, we invoke cataclysnıs to desolate the world, or invent laws on the duration of the forms of life!"”

The effect of the struggle for existence in determining the distribution of species is well illustrated in the following cases :-

"As the species of the same genus usually have, though by no means invariably, much similarity in habits and con- 
stitution, and always in structure, the struggle will generally be more severe between them, if they come into competition with each other, than between the species of distinct genera. We see this in the recent extension over parts of the United States of one species of swallow having caused the decrease of another species. The recent increase of the missel-thrush in parts of Scotland has caused the decrease of the songthrush. How frequently we hear of one species of rat taking the place of another species under the most different climates! In Russia the small Asiatic cockroach has everywhere driven before it its great congener. In Australia the imported hive-bee is rapidly exterminating the small, stingless native bee. One species of charlock has been known to supplant another species; and so in other cases. We can dimly see why the competition should be most severe between allied forms, which fill nearly the same place in the economy of nature; but probably in no one case could we precisely say why one species has been victorious over another in the great battle of life."

All this goes to show, if it really shows anything at all, that the distribution of a species is determined, in part, by its relation to other animals and plants - a truism that is recognized by every naturalist. The statement has no necessary bearing on the origin of new species through competition, as the incautious reader might infer. Not that I mean in any way to imply that Darwin intended to produce this effect on the reader; but Darwin is not always careful to discriminate as to the full bearing of the interesting illustrations with which his book so richly abounds.

At the end of his treatment of the subject, Darwin emphasizes once more how little we know about the subject of the struggle for existence.

"It is good thus to try in imagination to give to any one species an advantage over another. Probably in no single instance should we know what to do. This ought to con- 
vince us of our ignorance on the mutual relations of all organic beings; a conviction as necessary, as it is difficult, to acquire. All that we can do, is to keep steadily in mind that each organic being is striving to increase in a geometrical ratio; that each at some period of its life, during some season of the year, during each generation or at intervals, has to struggle for life and to suffer great destruction. When we reflect on this struggle, we may console ourselves with the full belief, that the war of nature is not incessant, that no fear is felt, that death is generally prompt, and that the vigorous, the healthy, and the happy survive and multiply."

The kindliness of heart that prompted the concluding sentence may arouse our admiration for the humanity of the writer, but need not, therefore, dull our criticism of his theory. For whether no fear is felt, and whether death is prompt or slow, has no bearing on the question at issue - except as it prepares the gentle reader to accept the dreadful calamity of nature, pictured in this battle for existence, and make more contented with their lot "the vigorous, the healthy, and the bappy."

\section{The Theory of Natural Selection}

We have already anticipated, to some extent, Darwin's conclusion in regard to the outcome of the competition of animals and plants. This result is supposed to lead to the survival of the fittest. The competition is carried out by nature, who is personified as selecting those forms for further experiments that have won in the struggle for existence.

"Can the principle of selection, which we have seen is so potent in the hands of man, apply under Nature? I think we shall see that it can act most efficiently. Let the endless number of slight variations and individual differences occurring in our domestic productions, and, in a lesser degree, in those under Nature, be borne in mind; as well as the strength of the hereditary tendency. Can it, then, be thought im- 
probable, seeing that variations useful to man have undoubtedly occurred, that other variations useful in some way to each being in the great and complex battle for life, should occur in the course of many successive generations? If such do occur can we doubt (remembering how many more individuals are born than can possibly survive) that individuals having any advantage, however slight, over others, would have the best chance of surviving and of procreating their kind? On the other hand, we may feel sure that any variation in the least degree injurious would be rigidly destroyed."

The process of natural selection is defined as follows, "The preservation of favorable individual differences and variations and the destruction of those that are injurious I have called Natural Selection or the Survival of the Fittest." And immediately there follows the significant statement, that, "Variations neither useful nor injurious would not be affected by natural selection, and would be left either a fluctuating element, as perhaps we see in certain polymorphic species, or would ultimately become fixed, owing to the nature of the organism and the nature of the conditions." It will be seen from this quotation, as well as from others already given, that Darwin leaves many structures outside of the pale of natural selection, and uses his theory to explain only those cases that are of sufficient use to be decisive in the life and death struggle of the individuals with each other and with the surrounding conditions.

Darwin states that we can best understand "the probable course of natural selection by taking the case of a country undergoing some slight physical change, for instance, of climate. The proportional numbers of its inhabitants will almost immediately undergo a change, and some species will probably become extinct. We may conclude, from what we have seen of the intimate and complex manner in which the inhabitants of each country are bound together, that any change in the numerical proportions of the inhabitants, in- 
dependently of the change of climate itself, would seriously affect the others. . . I In such cases, slight modifications, which in any way favored the individuals of any species, by better adapting them to their altered conditions, would tend to be preserved; and natural selection would have free scope for the work of improvement."

The first half of the first of these two quotations seems so plausible, that without further thought we may be tempted to give a ready assent to the second, yet the whole issue is contained in this statement. In the abstract, it undoubtedly appears true that any slightly useful modification might tend to be preserved. Whether it will, in reality, be preserved must depend on many things that should be taken into account. This question will come up later for further consideration; but it should be pointed out here, that, eren assuming that one or more individuals happen to possess a favorable variation, it by no means follows that natural selection would have free scope for the work of improvement, because the question of the inheritance of this variation, and of its accumulation and building up through successive generations, must be determined before we can be expected to give assent to this argument, that appears so attractive when stated in an abstract and vague way.

Darwin again makes the statement that under the term adration it must never be forgotten that mere individual differences are meant. "As a man can produce a great result with his domestic animals and plants by adding up in any given direction individual differences, so could natural selection, but far more easily from having incomparably longer time for action." Too much emphasis cannot be laid on the fact that Darwin believed that selection takes place amongst the small individual differences that we find in animals and plants. Some of his followers, as we shall see, are apt to put into the background this fundamental conception of Darwin's view. His constant comparison between 
the results of artificial and natural selection leaves no room for doubt as to his meaning. Darwin himself seems, at times, not unconscious of the weakness of this comparison. He says: "How fleeting are the wishes and efforts of man! how short his time! and consequently how poor will be his results, compared with those accumulated by Nature during whole geological periods. Can we wonder then that Nature's productions should be far 'truer' in character than man's productions; that they should be infinitely better adapted to the most complex conditions of life, and should plainly bear the stamp of far higher workmanship?" We should not lose sight of the fact that even after the most rigorous selective process has been brought to bear on organisms, namely, by isolation under domestication, we do not apparently find ourselves gradually approaching nearer and nearer to the formation of new species, but we find, on the contrary, that we have produced something quite different. In the light of this truth, the relation between the two selective theories may appear quite different from the interpretation that Darwin gives of it. We may well doubt whether nature does select so much better than does man, and whether she has ever made new species in this way.

We come now to a point that touches the theory of natural selection in a very vital spot.

"It may be well here to remark that with all beings there must be much fortuitous destruction, which can have little or no influence on the course of natural selection. For instance, a vast number of eggs or seeds are annually devoured, and these could be modified through natural selection only if they varied in some manner which protected them from their enemies. Yet many of these eggs or seeds would perhaps, if not destroyed, have yielded individuals better adapted to their conditions of life than any of those which happened to survive. So again a vast number of mature animals and plants, whether or not they be the best adapted to their conditions, 
must be annually destroyed by accidental causes, which would not be in the least degree mitigated by certain changes of structure or constitution which would in other ways be beneficial to the species. But let the destruction of the adults be ever so heavy, if the number which can exist in any district be not wholly kept down by such causes, - or again let the destruction of eggs or seeds be so great that only a hundredth or a thousandth part are developed, - yet of those which do survive, the best adapted individuals, supposing that there is any variability in a favorable direction, will tend to propagate their kind in larger numbers than the less well adapted. If the numbers be wholly kept down by the causes just indicated, as will often have been the case, natural selection will be powerless in certain beneficial directions; but this is no valid objection to its efficiency at other times and in other ways; for we are far from having any reason to suppose that many species ever undergo modification and improvement at the same time in the same area."

Some of the admissions made in this paragraph have an important bearing on the theory of natural selection. Far from supposing that fortuitous destruction would have no influence on the course of natural selection, it can be shown that it would have a most disastrous effect. In many cases the destruction comes in the form of a catastrophe to the individuals, so that small differences in structure, whether advantageous or not, are utterly unavailing. Our experience shows us that a destruction of this sort is going on around us all the time, and accounts in large part for the way in which the majority of animals and plants are clestroyed. Unless, for example, a seed happen to fall on a place suitable for its growth, it will perish without respect to a slight advantage it may have over other seeds of its kind. Of the thousands of eggs laid by one starfish, chance alone will decide whether one or another embryo is destroyed by larger animals, or if they escape this danger, the majority of them may be 
carried out to sea, where it will not be of the least avail if one individual has a slight advantage over the others. Darwin admits this, but adds that, if only a thousandth part is developed, yet of those that do survive the best adapted individuals will tend to propagate their kind in largersnumbers than the less well adapted. The argument is not, however, so simple as it appears to be on the surface. I pass over, for the present, the apparent inconsequence in this statement that the best adapted individuals will tend to propagate their kind in larger numbers. It is not by any means certain that this is the case. Darwin's meaning is, however, fairly clear, and can be interpreted to mean this: after the fortuitous destruction has finished, there will be a further competition of the survivors amongst themselves and with the surrounding conditions. In this higher competition, which is less severe, small individual differences suffice to determine the survival of certain individuals. These are, therefore, selected.

In this argument it is assumed that a second competition takes place after the first destruction of individuals has occurred, and this presupposes that more individuals reach maturity than there is room for in the economy of nature. But we do not know to what extent this takes place. If only as many mature as can survive, then the second competition does not take place. If, on the other hand, fewer mature than there is room for, then again competition does not take place. And if at all times selection is not rigorously carried out, everything may be lost that has been so laboriously gained. We see then that the result that Darwin imagines would take place, can be carried out only when more individuals reach maturity than there is room for (if it is a case of competition with one another), or that escape their enemies (if it is a question of competition with other forms).

It is instructive to consider some of the examples that Darwin has given to illustrate how the process of natural 
selection is carried out. The first example is the imaginary case of a species of wolf, the individuals of which secure their prey sometimes by craft, sometimes by strength, and sometimes by fleetness. If the prey captured by the first two methods should fail, then all the wolves would be obliged to capture their food by fleetness, and consequently the fleetest alone would survive. "I can see no more reason to doubt that this would be the result than that man should improve the fleetness of his greyhounds." But even if the fleetness of the race could be kept up in this way, it does not follow that a new species of wolf would be formed in consequence, as Darwin implies. His own comment on this illustration is, perhaps, the best criticism that can be made.

"It should be observed that, in the above illustration, I speak of the slimmest individual wolves, and not of any single strongly marked variation having been preserved. In former editions of this work I sometimes spoke as if this latter alternative had frequently occurred. I saw the great importance of individual differences, and this led me fully to discuss the results of unconscious selection by man, which depends on the preservation of all the more or less valuable individuals, and on the destruction of the worst. I saw, also, that the preservation in a state of nature of any occasional deviation of structure, such as a monstrosity, would be a rare event ; and that, if at first preserved, it would generally be lost by subsequent intercrossing with ordinary individuals. Nevertheless, until reading an able and valuable article in the North British Revicw (1867), I did not appreciate how rarely single variations, whether slight or strongly marked, could be perpetuated. The author takes the case of a pair of animals, producing during their lifetime two hundred offspring, of which, from various causes of destruction, only two on an average survive to procreate their kind. This is rather an extreme estimate for most of the higher animals, but by no means so for many of the lower organisms. He then 
shows that if a single individual were born, which varied in some manner, giving it twice as good a chance of life as that of the other individuals, yet the chances would be strongly against its survival. Supposing it to survive and to breed, and that half its young inherited the favourable variation; still, as the reviewer goes on to show, the young would have only a slightly better chance of surviving and breeding; and this chance would go on decreasing in the succeeding generations. The justice of these remarks cannot, I think, be disputed. If, for instance, a bird of some kind could procure its food more easily by having its beak curved, and if one were born with its beak strongly curved, and which consequently flourished, nevertheless there would be a very poor chance of this one individual perpetuating its kind to the exclusion of the common form; but there can hardly be a doubt, juclging by what we see taking place under domestication, that this result would follow from the preservation during many generations of a large number of individuals with more or less strongly curved beaks, and from the destruction of a still larger number with the straightest beaks."

There then follows what, I believe, is one of the most significant admissions in the "Origin of Species":-

"It should not, however, be overlooked that certain rather strongly marked variations, which no one would rank as mere individual differences, frequently recur owing to a similar organization being similarly acted on - of which fact numerous instances could be given with our domestic productions. In such cases, if the varying individual did not actually transmit to its offspring its newly acquired character, it would undoubtedly transmit to them, as long as the existing conditions remained the same, a still stronger tendency to vary in the same manner. There can also be little doubt that the tendency to vary in the same manner has often been so strong that all the individuals of the same species have been similarly modified without the aid of any form of selection. 
Or only a third, fifth, or tenth part of the individuals may have been thus affected, of which fact several instances could be given. Thus Graba estimates that about one-fifth of the guillemots in the Faroe Islands consist of a variety so well marked, that it was formerly ranked as a distinct species under the name of Uria lacrymans. In cases of this kind, if the variation were of a beneficial nature, the original form would soon be supplanted by the modified form, through the survival of the fittest."

Do not the admissions in this paragraph almost amount to a withdrawal of much that has preceded in regard to the survival of fluctuating, individual differences? In the last edition, from which we have just quoted, Darwin, in response to the criticisms which his book met, inserted here and there statements that are in many ways in contradiction to the statements in the first edition, and yet the earlier statements have been allowed to stand for the most part.

The next example is also worthy of careful examination, since it appears to prove too much :

"It may be worth while to give another and more complex illustration of the action of natural selection. Certain plants excrete sweet juice, apparently for the sake of eliminating something injurious from the sap: this is effected, for instance, by glands at the base of the stipules in some Leguminosx, and at the backs of the leaves of the common laurel. This juice, though small in quantity, is greedily sought by insects; but their visits do not in any way benefit the plant. Now, let us suppose that the juice or nectar was excreted from the inside of the flowers of a certain number of plants of any species. Insects in seeking the nectar would get dusted with pollen, and would often transport it from one flower to another. The flowers of two distinct individuals of the same species would thus get crossed; the act of crossing; as can be fully proved, gives rise to vigorous seedlings, which consequently would have the best chance of flourish- 
ing and surviving. The plants which produced flowers with the largest glands or nectaries, excreting most nectar, would oftenest be visited by insects, and would oftenest be crossed; and so in the long run would gain the upper hand and form a local variety.'

The reader will notice that the sweet juice or nectar secreted by certain plants is supposed to have first appeared independently of the action of natural selection. Why then account for its presence in flowers as the outcome of an entirely different process? If the nectar is eagerly sought for by insects, without the plant benefiting in any way by their visitations, why give a different explanation of its origin in flowers where it is of benefit to the plant?

Darwin carries his illustration further: "When our plant, by the above process long continued, had been rendered highly attractive to insects, they would unintentionally, on their part, regularly carry pollen from flower to flower; and that they do this effectually, I could easily show by many striking facts. I will give only one, as likewise illustrating one step in the separation of the sexes of plants. ... As soon as the plant had been rendered so highly attractive to insects that pollen was regularly carried from flower to flower, another process might commence. No naturalist cloubts the advantage of what has been called the 'physiological division of labour'; hence we may believe that it would be advantageous to a plant to produce stamens alone in one flower or on one whole plant, and pistils alone in another flower or on another plant. In plants under culture and placed under new conditions of life, sometimes the male organs and sometimes the female organs become more or less impotent; now if we suppose this to occur in ever so slight a degree under nature, then, as pollen is already carried regularly from flower to flower, and as a more complete separation of the sexes of our plant would be advantageous on the principle of the division of labour, individuals with this tendency 
more and more increased would be continually favoured or selected, until at last a complete separation of the sexes might be effected. It would take up too much space to show the various steps, through dimorphism and other means, by which the separation of the sexes in plants of various kinds is apparently now in progress; but I may add that some of the species of holly in North America are, according to Asa Gray, in an exactly intermediate condition, or, as he expresses it, are more or less diøeciously polygamous."

From this it will be secn that Darwin supposes that the separation of the sexes in some of the higher plants has been brought about by natural selection. Despite the supposed advantage of the so-called "clivision of labor," one may, I venture to suggest, be sceptical as to whether the separation of the sexes can be explained in this way. The whole case is largely supposititious, since in most of the higher hermaphroditic plants and in nearly all hermaphroditic animals the sexual products ripen at different times in the same individual. Hence there is no basis for the assumption that unless the sexes are separated there will be self-fertilization. Shall we assume that this difference in time of ripening of the two kinds of sex-cells is also the outcome of natural selection, and that there has existed an earlier stage in all animals and plants, that now have different times for the ripening of their sexual elements, a time when these products ripened simultaneously? I doubt if even a Darwinian would give such loose rein to his fancy.

But this is not yet the whole story that Darwin has made out in this connection, for he continues:-

"Let us now turn to the nectar-feeding insects; we may suppose the plant, of which we have been slowly increasing the nectar by continued selection, to be a common plant; and that certain insects depended in main part on its nectar for food. I could give many facts showing how anxious bees 
are to save time: for instance, their habit of cutting holes and sucking the nectar at the bases of certain flowers, which with a very little more trouble, they can enter by the mouth. Bearing such facts in mind, it may be believed that under certain circumstances individual differences in the curvature or length of the proboscis, etc., too slight to be appreciated by us, might profit a bee or other insect, so that certain individuals would be able to obtain their food more quickly than others: and thus the communities to which they belonged would flourish and throw off many swarms inheriting the same peculiarities."

Aside from the general criticism that will suggest itself here also, it should be pointed out that even if "certain individuals" of the bees had slightly longer proboscides, this would, in the case of the hive-bees at least, be of no avail, since they do not reproduce, and hence leave no descendants with longer mouth-parts. Of course, it may be replied that those colonies in which the queens produce more of the longproboscis kind of worker would have an advantage over other colonies not having so many individuals of this sort. It would then be a competition of one colony with another, as Darwin supposes to take place in colonial forms. But whether slight differences of this sort would lead to the elimination of the least well-endowed colonies is entirely a matter of speculation. Since there are flowers with corolla-tubes of all lengths, we can readily suppose that if one kind of flower excluded individuals of certain colonies, they would search elsewhere for their nectar rather than perish. While different races might arise in this way, the process would not be the survival of the fittest, but a process of adaptation to a new environment.

We come now to a topic on which Darwin lays much stress: the divergence of character. He tries to show how the "lesser differences between the varieties become augmented into the greater differences between species." 
"Mere chance, as we may call it, might cause one variety to differ in some character from its parents, and the offspring of this variety again to differ from its parent in the very same character and in a greater degree; but this alone would never account for so habitual and large a degree of difference as that between the species of the same genus. As has always been my practice, I have sought light on this head from our domestic productions."

Then, after pointing out that under domestrcation two different races, the race-horse and the dray-horse, for instance, might arise by selecting different sorts of variations, Darwin inquires:-

"But how, it may be asked, can any analogous principle apply in nature? I believe it can and does apply most efficiently (though it was a long time before I saw how), from the simple circumstance that the more diversified the descendants from any one species become in structure, constitution, and habits, by so much will they be better enabled to seize on many and widely diversified places in the polity of nature, and so be enabled to increase in numbers."

Here we touch on one of the fundamental principles of the doctrine of evolution. It is intimated that the new form of animal or plant first appears (without regard to any kind of selection), and then finds that place in nature where it can remain in existence and propagate its kind. Darwin refers here, of course, only to the less extensive variations, the individual or fluctuating kind; but as we shall discuss at greater length in another place, this same process, if extended to other kinds of variation, may give us an explanation of evolution without competition, or selection, or destruction of the individuals of the same kind taking place at all. 


\section{CHAPTER V}

\section{THE THEORY OF NATURAL SELECTION (Continued)}

\section{Objections to the Theory of Natural Selection}

Although in the preceding chapter a number of criticisms have been made of the special parts of the theory of natural selection, there still remain to be considered some further objections that have been made since the first publication of the theory. It is a fortunate circumstance from every point of view that Darwin himself was able in the later editions of the "Origin of Species" to reply to those criticisms that he thought of sufficient importance. He says :-

"Long before the reader has arrived at this part of my work, a crowd of difficulties will have occurred to him. Some of them are so serious that to this day I can hardly reflect on them without being in some degree staggered; but, to the best of my judgment, the greater number are only apparent, and those that are real are not, I think, fatal to the theory."

The first difficulty is this: "Why, if species have descended from other species by fine gradations, do we not everywhere see innumerable transitional forms? Why is not all nature in confusion, instead of the species being, as we see them, well defined?"

The answer that Darwin gives is, that by competition the new form will crowd out its own less-improved parent form, and other less-favored forms. But is this a sufficient or satisfactory answer? If we recall what Darwin has said on the advantage that those forms will have, in which a great number of new variations appear to fit them to the great diversity 
of natural conditions, and if we recall the gradations that exist in external conditions, I think we shall find that Darwin's reply fails to give a satisfactory answer to the question.

It is well known, and Darwin himself has commented on it, that the same species often remains constant under very diverse external conditions, both inorganic and organic. Hence I think the explanation fails, in so far as it is based on the accumulation by selection of small individual variations that are supposed to give the individuals some slight advantage under each set of external conditions. Darwin admits that "this difficulty for a long time quite confounded me. But I think it can be in large part explained." The first explanation that is offered is that areas now continuous may not have been so in the past. This may be true in places, but the great continents have had continuous areas for a long time, and Darwin frankly acknowledges that he "will pass over this way of explaining the difficulty." The second attempt is based on the supposed narrowness of the arca, where two species, descended from a common parent, overlap. In this region the change is often very abrupt, and Darwin adds:-

"To those who look at climate and the physical conditions of life as the all-important elements of distribution, these facts ought to cause surprise, as climate and height or depth graduate away insensibly. But when we bear in mind that almost every species, even in its metropolis, would increase immensely in numbers, were it not for other competing species; that nearly all either prey on or serve as prey for others; in short, that each organic being is either directly or indirectly related in the most important manner to other organic beings, - we see that the range of the inhabitants of any country by no means exclusively depends on insensibly changing physical conditions, but in a large part on the presence of other species, on which it lives, or by which it is destroyed, or with which it comes into competition; and as these species are already defined ob- 
jects, not blending one into another by insensible gradations, the range of any one species, depending as it does on the range of others, will tend to be sharply defined."

Here we have a petitio principii. The sharp definition of species, that we started out to account for, is explained by the sharp clefinition of other species!

A third part of the explanation is that, owing to the relative fewness of individuals at the confines of the range during the fluctuations of their enemies, or of their prey, or in the nature of the seasons, they would be extremely liable to utter extermination. If this were really the case, then new species themselves which, on the theory, are at first few in numbers ought to be exterminated. On the whole, then, it does not appear that Darwin has been very successful in his attempt to meet this objection to the theory.

Darwin tries to meet the objection, that organs of extreme perfection and complication cannot be accounted for by natural selection, as follows:-

"To suppose that the eye with all its inimitable contrivances for adjusting the focus to different distances, for admitting different amounts of light, and for the correction of spherical and chromatic aberration, could have been formed by natural selection, seems, I freely confess, absurd in the highest degree."

The following sketch that Darwin gives to show how he imagined the vertebrate eye to have been formed is very instructive, as illustrating how he supposed that natural selection acts :--

"If we must compare the eye to an optical instrument, we ought in imagination to take a thick layer of transparent tissue, with spaces filled with fluid, and with a nerve sensitive to light beneath, and then suppose every part of this layer to be continually changing slowly in density, so as to separate into layers of different densities and thicknesses, placed at different distances from each other, and with the 
surfaces of each layer slowly changing in form. Further we must suppose that there is a power, represented by natural selection or the survival of the fittest, always intently watching each slight alteration in the transparent layers; and carefully preserving each which, under varied circumstances, in any way or in any degree, tends to produce a distincter image. We must suppose each new state of the instrument to be multiplied by the million; each to be preserved until a better one is produced, and then the old ones to be all destroyed. In living bodies, variation will cause the slight alterations, generation will multiply them almost infinitely, and natural selection will pick out with unerring skill each improvement. Let this process go on for millions of years; and during each year on millions of individuals of many kinds; and may we not believe that a living optical instrument might thus be formed as superior to one of ghass, as the works of the Creator are to those of man."

We may conelude in Darwin's own words:-

"To arrive, however, at a just conclusion regarding the formation of the eyc, with all its marvellous yet not absolutely perfect characters, it is indispensable that the reason should conquer the imagination; but I have felt the difficulty far too keenly to be surprised at others hesitating to extend the principle of natural selection to so startling a length."

The electric organs, present in several fish, offer a case of special diffieulty to the selection theory. When well developed, as in the Torpeclo and in Gymnotus, it is conceivable that it may serve as an organ of defence, but in other forms the shock is so weak that it is not to be supposed that it can have any such function. Romanes, who in many ways was one of the stanchest followers of Darwin, admits that, so far as he can see, the evolution of the clectric organs cannot be explained by the selection theory. Darwin offers no cxplanation, but bases his defence on the grounds that we do not know of what use this organ can be to the animal. 
Darwin also refers to the phosphorescent, or luminous, organs as a supposed case of difficulty for his theory.

"The luminous organs which occur in a few insects, belonging to widely different families, and which are situated in different parts of the body, offer, under our present state of ignorance, a difficulty almost exactly parallel with that of the electric organs."

In this case also, as in that of the electric organs, the structures appear in entirely different parts of the body of the insect in different species, so that their occurrence in this group cannot be accounted for on a common descent. In whatever way they have arisen, they must have evolved independently in different species. Darwin advances no explanation of the origin of the luminous organs, but states that they "offer under our present state of ignorance a diffculty almost exactly parallel with that of the electric organs." It will be noticed that the difficulty referred to rests on the assumption that since the organs are well developed they must have some important use!

We may next consider "organs of little apparent importance as affected by natural selection." Darwin says :-

"As natural selection acts by life and death, - by the survival of the fittest, and by the destruction of the less wellfitted individuals, - I have sometimes felt great difficulty in understanding the origin or formation of parts of little importance; almost as great, though of a very different kind, as in the case of the most perfect and complex organs." .

His answers to this difficulty are: (I) we are too ignorant "in regarl to the whole economy of any one organic being to say what slight modifications would be of importance or not," - thus such apparently trifling characters as the down on fruit, or the colors of the skin and hair of quadrupeds, which from being correlated with constitutional differences or from determining the attacks of insects might be acted on by natural selection; (2) organs now of trifling importance have in 
some cases been of high importance to an early progenitor; (3) the changed conditions of life may account for some of the useless organs; (4) reversion accounts for others; (5) the complex laws of growth account for still others, such as correlation, compensation of the pressure of one part on another, etc.; (6) the action of sexual selection is responsible for many characters not to be explained by natural selection. Admitting that there may be cases that can be accounted for on one or the other of these six possibilities, yet there can be no doubt that there are still a considerable number of specific characters that cannot be explained in any of these ways. I do not think that Darwin has by any means met this objection, even if all these six possibilities be admitted as generally valid.

Amongst the "miscellaneous objections" to his theory that Darwin considers we may select the most important cases. The following paragraph has been sometimes quoted by later writers to show that Darwin saw, to a certain extent, the insufficiency of fluctuating variations as a basis for selection. What he calls here "spontaneous variability" refers to sudden and extensive variations, or what we may call discontinuous variations. "In the earlier editions of this work I underrated, as it now seems probable, the frequency and importance of modifications due to spontaneous variability. But it is impossible to attribute to this cause the innumerable structures which are so well adapted to the habits of life of each species. I can no more believe in this, that the well-adapted form of a race-horse or greyhound, which before the principle of selection by man was well understood, excited so much surprise in the minds of the older naturalists, can thus be explained."

Darwin appears to mean by the latter part of this statement, that he camot believe that such sudden and great variations as have caused a peach tree to produce nectarines can account for the wonderful adaptations of organisms; but 


\section{Darwin's Artificial and Natural Selection 135}

it is not really necessary to suppose that this would often occur, for the same result could be reached by several stages, even if the discontinuous variations had been small, and had appeared in many individuals simultaneously. After showing that in a number of flowers, especially of the Compositxe and Umbellifera, the individual flowers in the closely crowded heads are sometimes formed on a different type, Darwin concludes: "In these several cases, with the exception of that of the well-developed ray-florets, which are of service in making the flowers conspicuous to insects, natural selection cannot, as far as we can judge, have come into play, or only in a quite subordinate manner. All these modifications follow from the relative position and interaction of the parts; and it can hardly be doubted that if all the flowers and leaves on the same plant had been subjected to the same external and internal condition, as are the flowers and leaves in certain positions, all would have been modified in the same manner."

Further on we meet with the following remarkable statement: "But when, from the nature of the organism and of the conditions, modifications have been induced which are unimportant for the welfare of the species, they may be, and apparently often have been, transmitted in nearly the same state to numerous, otherwise modified, descendants. It cannot have been of much importance to the greater number of mammals, birds, or reptiles, whether they were clothed with hair, feathers, or scales; yet hair has been transmitted to almost all mammals, feathers to all birds, and scales to all true reptiles. A structure, whatever it may be, which is common to many allied forms, is ranked by us as of high systematic importance, and consequently is often assumed to be of high vital importance to the species. Thus, as I am inclined to believe, morphological differences, which we consider as important, - such as the arrangement of the leaves, the divisions of the flower or of the ovarium, the position of the ovules, etc., - first appeared in many cases as fluctuating 
variations, which sooner or later became constant through the nature of the organism and of the surrounding conditions, as well as through the intercrossing of distinct individuals, but not through natural selection; for as these morphological characters do not affect the welfare of the species, any slight deviations in them could not have been governed or accumulated through this latter agency. It is a strange result which we thus arrive at, namely, that characters of slight vital importance to the species are the most important to the systematist; but, as we shall hereafter see when we treat of the genetic principle of classification, this is by no means so paradoxical as it may at first appear."

If all this be granted, it is once more evident that the only variations that come under the action of selection are the limited number that are of vital importance to the organism. How little the theory of natural selection can be used to explain the origin of species will be apparent from the above quotation. This is, of course, not an argument against the theory itself, which would still be one of vast importance if it explained adaptive characters alone; but enough has been said, I think, to show that it is improbable that the origin of adaptive and non-adaptive characters are to be explained by entirely different principles.

In reply to a criticism of Mivart, Darwin makes the further admission as to the insufficiency of the theory of natural selection: "When discussing special cases, Mr. Mivart passes over the effects of the increased use and disuse of parts, which I have always maintained to be highly important, and have treated in my "Variation under Domestication' at greater length than, as I believe, any other writer. He likewise often assumes that I attribute nothing to variation, inclependent of natural selection, whereas in the work just referred to I have collected a greater number of well-established cases than is to be found in any other work known to me." If this is admitted, and if it can be shown 
that the evidence in favor of the inheritance of acquired characters is very doubtful at best, may we not conclude that Mivart's criticisms have sometimes hit the mark?

The following objection appears to be a veritable stumbling-block to the theory. Flatfishes and soles lie on one side, and do not stand in a vertical position as do other fish. Some species lie on one side and some on the other, and some species contain both right-sided and left-sided individuals. In connection with this unusual habit we find a striking change in the structure. The eye that would be on the under side has shifted, so that it has come to lie on the upper side of the head, i.c. both eyes lie on the same side, a condition found in no other vertebrate. As a result of the shifting of the eye, the bones of the skull have also become profoundly modified. The young fish that emerge from the egg swim at first upright, as do ordinary fish, and only after they have led a free existence for some time do they turn to one side and sink to the bottom. Unless the under eye moved to the upper side it would be of no use to the flatfish, and might even be a source of injury. Mivart points out that a sudden, spontaneous transformation in the position of eye is hardly conceivable, and to this Darwin, of course, assents. Mivart adds: "If the transit was gradual, then how such transit of one eye a minute fraction of the journey towards the other side of the head could benefit the individual is, indeed, far from clear. It seems even that such an incipient transformation must rather have been injurious." Darwin's reply is characteristic:-

"We thus see that the first stages of the transit of the eye from one side of the head to the other, which Mr. Mivart considers would be injurious, may be attributed to the habit, no doubt beneficial to the individual and to the species, of endeavoring to look upwards with both eyes, whilst resting on one side at the bottom. We may also attribute to the inherited effects of use the fact of the mouth in several kinds 
of flatfish being bent towards the lower surface, with the jawbones stronger and more effective on this, the eyeless side of the head, than on the other side, for the sake, as Dr. Traquair supposes, of feeding with ease on the ground. Disuse, on the other hand, will account for the less developed condition of the whole inferior half of the body, including the lateral fins; though Yarrell thinks that the reduced size of these fins is advantageous to the fish, as 'there is so much less room for their action, than with the larger fins above.' Perhaps the lesser number of teeth in the proportion of four to seven in the upper halves of the two jaws of the plaice, to twentyfive to thirty in the lower halves, may likewise be accounted for by disuse. From the colorless state of the ventral surface of most fishes and of many other animals, we may reasonably suppose that the absence of color in flatfish on the side, whether it be the right or left, which is undermost, is due to the exclusion of light."

By falling back on the theory of inheritance of acquired characters Darwin tacitly admits the incompetence of natural selection to explain the evolution of the flatfish. If the latter theory prove incorrect, it must then be admitted that the erolution of the flatfishes cannot be accounted for by either of the two main theories on which Darwin relies.

Mivart further points out that the beginning stages of the mammary glands cannot be explained by Darwin's theory. To which Darwin replies, that an American naturalist, Mr. Lockwood, believes from what he has seen of the development of the young of the pipe-fish (Hippocampus) that "they are nourished by a secretion from the cutaneous glands of the sac" in which the young are enclosed. This can scarcely be said to be a satisfactory reply; for, if it is true that this is the case for the pipe-fish, - and I cannot find on inquiry that this statement has been confirmed, - it is still rather speculative to suppose that the ancestral mammals nourished their young by secreting a fluid into the marsupial sac around the embryos. 
Darwin deals with instincts of animals in the same way as he deals with their structures. After pointing out that instincts are variable, and that the variations are hereditary, he proceeds to show how selection may act by picking out those individuals possessing the more favorable instincts. In other words, the theory of natural selection is applied to functions, as well as to structure. Darwin makes use here also of the Lamarckian factor of inheritance, and concludes that "in most cases habit and selection have probably both occurred."

A few examples will sufficiently serve to illustrate Darwin's meaning. The first case given is that of the cuckoo, which lays its eggs in the nests of other birds, where they are hatched and the young reared by their foster-parents. The starting-point for such a perversion of the ordinary habits of birds is to be found, he thinks, in the occasional deposition of eggs in the nests of other birds, which has at times been observed for a number of species. For instance, this has been seen in the American cuckoo, which ordinarily builds a nest of its own. It is recorded and believed to be true that the young English cuckoo, when only two or three days old, ejects from the nest the offspring of its foster-parents, and this "strange and odious instinct " is supposed by Darwin to have been acquired in order that the young cuckoo might get more food, and that the young bird has acquired during successive generations the strength and structure necessary for the work of ejection. This is of course largely speculative, and it is by no means obvious that it was a greater benefit to the cuckoo to have other birds rear its young than to do so itself. We can equally well imagine, since this is the turn the argument takes, that the occasional instinct to deposit eggs in the nests of other birds would be disadvantageous, and could not have been acquired by the selection of a fluctuating instinct of this sort. We have no right to assume, that because a new habit has been acquired, that it is a more advantageous one than the one that has been lost. All that we can legit- 
imately infer is, that, although the normal instinct has been changed into another, the race has still been able to remain in existence. The same conclusion applies to the case of Molothrus bonaricnsis, cited by Darwin, and is here even more obvious:-

"Some species of Molothrus, a widely distinct genus of American birds, allied to our starlings, have parasitic habits like those of the cuckoo; and the species present an interesting gradation in the perfection of their instincts. The sexes of Molothrus badius are stated by an excellent observer, $\mathrm{Mr}$. Hudson, sometimes to live promiscuously together in flocks, and sometimes to pair. They either build a nest of their own, or seize on one belonging to some other bircl, occasionally throwing out the nestlings of the stranger. They either lay their eggs in the nest thus appropriated, or oddly enough build one for themselves on the top of it. They usually sit on their own eggs and rear their own young; but Mr. Hudson says it is probable that they are occasionally parasitic, for he has seen the young of this species following old birds of a distinct kind and clamoring to be fed by them. The parasitic habits of another species of Molothrus, the MI. bonariensis, are much more highly developed than those of the last, but are still far from perfect. This bird, as far as is known, invariably lays its eggs in the nest of strangers; but it is remarkable that several together sometimes commence to build an irregular untidy nest of their own, placed in singularly ill-adapted situations, as on the leaves of a large thistle. They never, however, as far as Mr. Hudson has ascertained, complete a nest for themselves. They often lay so many egrs - from fifteen to twenty - in the same foster-nest, that few or none can possibly be hatched. They have, moreover, the extraordinary habit of pecking holes in the eggs, whether of their own species or of their foster-parents, which they find in the appropriated nests. They drop also many eggs on the bare ground, which are thus wasted." 


\section{Darwin's Artificial and Natural Selection}

Can we possibly be expected to believe that it has been to the advantage of this species to give up its original regular method of incubating its own eggs, and acquire such a haphazard, new method? Does not the explanation prove too much, rather than give support to Darwin's hypothesis? Is it not better to conclude, that despite the disadvantages entailed by a change in the original instincts, the species is still able to remain in existence?

Darwin points out, in the case of the slave-making ants, that the slave-making instinct may have arisen in the first instance by ants carrying pupæ, that they have captured, into their own nests. Later this habit might become fixed, and, finally, after passing through several stages of development, the ants might become absolutely dependent on their slares. It is also supposed that those colonies in which this instinct was better developed would survive in competition with other colonies of the same species on account of the supposed advantage of owning slaves. In this way natural selection steps in and perfects the process.

It is far from proven, or even made probable, that a species of ant that becomes gradually dependent on its slaves is more likely to survive than other colonies that are not so dependent. All we can be certain of is that with slaves they have still been able to maintain their own. Moreover, we must not forget that it is not enough to show that a particular habit might be useful to a species, but it should also be shown that it is of sufficient importance, at every stage of its evolution, to give a decisive advantage in the "struggle for existence." For unless a life and death struggle takes place between the different colonies, natural selection is powerless to bring about its supposed results. And who will be bold enough to affirm that the presence of slaves in a nest will give victory to that colony in competition with its neighbors? Has the history of mankind taught us that the slave-making countries have exterminated the 
countries without slaves? Is the question so simple as this? May not the degeneration of the masters more than compensate for the acquirement of slaves, and may not the loss of life in obtaining slaves more than counterbalance the advantage of the slaves after they are captured? In the face of these possibilities it is not surprising to find that Darwin, when summing up the chapter, makes the following admission: "I do not pretend that the facts in this chapter strengthen in any degree my theory; but none of the cases of difficulty, to the best of my judgment, annihilate it." Darwin, with his usual frankness, adds :-

"No doubt many instincts of very difficult explanation could be opposed to the theory of natural selection, - cases, in which we cannot see how an instinct could have originated; cases, in which no intermediate gradations are known to exist; cases of instincts of such trifling importance, that they could hardly have been acted on by natural selection; cases of instincts almost identically the same in animals so remote in the scale of nature, that we cannot account for their similarity by inheritance from a common progenitor, and consequently must believe that they were independently acquired through natural selection. I will not here enter on these several cases, but will confine myself to one special difficulty, which at first appeared to me insuperable, and actually fatal to the whole theory. I allude to the neuters or sterile females in insect communities; for these neuters often differ widely in instinct and in structure from both the males and fertile females, and yet, from being sterile, they cannot propagate their kind.

"The subject well deserves to be discussed at great length, but I will here take only a single case, that of working or sterile ants. IIow the workers have been rendered sterile is a difficulty; but not much greater than that of any other striking modification of structure; for it can be shown that some insects and other articulate animals in a state of nature 
occasionally become sterile; and if such insects had been social, and it had been profitable to the community that a number should have been annually born capable of work, but incapable of procreation, I can see no especial difficulty in this having been effected through natural selection. But I must pass over this preliminary difficulty. The great difficulty lies in the working ants differing widely from both the males and the fertile females in structure, as in the shape of the thorax, and in being destitute of wings and sometimes of eyes, and in instinct. As far as instinct alone is concerned, the wonderful difference in this respect between the workers and the perfect females, would have been better exemplified by the hive-bee. If a working ant or other neuter insect had been an ordinary animal, I should have unhesitatingly assumed that all its characters had been slowly acquired through natural selection; namely, by individuals having been born with slight profitable modifications, which were inherited by the offspring; and that these again varied and again were selected, and so onwards. But with the working ant we have an insect differing greatly from its parents, yet absolutely sterile; so that it could never have transmitted successively acquired modifications of structure or instinct to its progeny. It may well be asked, how is it possible to reconcile this case with the theory of natural selection?"

Darwin's answer is that the differences of structure are correlated with certain ages and with the two sexes, but this is obviously only shifting the difficulty, not meeting it. $\mathrm{He}$ concludes, "I can see no great difficulty in any character becoming correlated with the sterile condition of certain members of the insect communities, the difficulty lies in understanding how such correlated modifications of structure could have been slowly accumulated by natural selection." "This difficulty, though appearing insuperable, is lessened, or, as I believe, disappears, when it is remembered that selection 
may be applied to the family, as well as to the individual, and may thus give the desired end."

Darwin did not fail to see that there is a further difficulty even greater than the one just mentioned. He says: "But we have not as yet touched on the acme of the difficulty; namely, the fact that the neuters of several ants differ, not only from the fertile females and males, but from each other, sometimes to an almost incredible degree, and are thus divided into two or even three castes. The castes, moreover, do not commonly graduate into each other, but are perfectly well defined; being as distinct from each other as are any two species of the same genus, or rather as any two genera of the same family. Thus in Eciton, there are working and soldier neuters, with jaws and instincts extraordinarily different: in Cryptocerus, the workers of one caste alone carry a wonderful sort of shield on their heads, the use of which is quite unknown: in the Mexican Myrmecocystus, the workers of one caste never leave the nest; they are fed by the workers of another caste, and they have an enormously developed abdomen which secretes a sort of honey, supplying the place of that excreted by the aphides, or the domestic cattle as they may be called, which our European ants guard and imprison."

"It will indeed be thought that I have an overweening confidence in the principle of natural selection, when I do not admit that such wonderful and well-established facts at once annihilate the theory. In the simpler case of neuter insects all of one caste, which, as I believe, have been rendered different from the fertile males and females through natural selection, we may conclude from the analogy of ordinary variations, that the successive, slight, profitable modifications did not first arise in all the neuters in the same nest, but in some few alone; and that by the survival of the communities with females which produced most neuters having the advantageous modification, all the neuters ultimately came to be 


\section{Darwin's Artificial and Natural Selection 145}

thus characterized. According to this view we ought occasionally to find in the same nest neuter insects, presenting gradations of structure; and this we do find, even not rarely, considering how few neuter insects out of Europe have been carefully examined."

From this the conclusion is reached:-

"With these facts before me, I believe that natural selection, by acting on the fertile ants or parents, could form a species which should regularly produce neuters, all of large size with one form of jaw, or all of small size with widely different jaws; or lastly, and this is the greatest difficulty, one set of workers of one size and structure, and simultaneously another set of workers of a different size and structure; - a graduated series having first been formed, as in the case of the driver ant, and then the extreme forms having been produced in greater and greater numbers, through the survival of the parents which generated them, until none with an intermediate structure were produced.

"I have now explained how, as I believe, the wonderful fact of two distinctly defined castes of sterile workers existing in the same nest, both widely different from each other and from their parents, has originated. We can see how useful their production may have been to a social community of ants, on the same principle that the division of labor is useful to civilized man. Ants, however, work by inherited instincts and by inherited organs or tools, whilst man works by acquired knowledge and manufactured instruments. But I must confess, that, with all my faith in natural selection, I should never have anticipated that this principle could have been efficient in so high a degree, had not the case of these neuter insects led me to this conclusion. I have, therefore, discussed this case, at some little but wholly insufficient length, in order to show the power of natural selection, and likewise because this is by far the most serious special difficulty which my theory has encountered. The case, also, is 
very interesting, as it proves that with animals, as with plants, any amount of modification may be effected by the accumulation of numerous, slight, spontaneous variations, which are in any way profitable, without exercise or habit having been brought into play. For peculiar habits confined to the workers or sterile females, however long they might be followed, could not possibly affect the males and fertile females, which alone leave descendants. I am surprised that no one has hitherto advanced this demonstrative case of neuter insects, against the well-known doctrine of inherited habit, as advanced by Lamarck."

We may dissent at once from Darwin's statement which, he thinks, "proves that any amount of modification may be affected by the accumulation of numerous slight variations which are in any way profitable without exercise or habit having been brought into play" ; we may dissent if for no other reason than that this begs the whole point at issue, and is not proven. It does not follow because in some colonies all intermediate stages of neuters exist, that in other colonies, where no such intermediate stages are present, these have been slowly weeded out by natural selection, causing to disappear all colonies slightly below the mark. It is this that begs the question. Because we can imagine that intermediate stages between the different castes may have been present, it neither follows that such fluctuating variattions have been the basis for the evolution of the more sharply defined types, nor that the imagined advantage of such a change would have led through competition to the extermination of the other colonies. However much we may admire the skill with which Darwin tried to meet this difficulty, let us not put down the results to the good of the theory, but rather repeat once more Darwin's own words at the end of this chapter, to the effect that the facts do not strengthen the theory. 


\section{Sterility between Species}

The care with which Darwin examined every bearing of his theory is nowhere better exemplified than in his treatment of the question of sterility between the individuals of different species. It would be so obviously to the advantage of the selection theory if it were true that sterility between species had been acquired by selection in order to prevent intercrossing, that it would have been easy for a less cautious thinker to have fallen into the error of supposing that sterility might have been acquired in this way. Tempting as such a view appears, Darwin was not caught by the specious argument, as the opening sentence in the chapter of hybridism shows:-

"The view commonly entertained by naturalists is that species, when intercrossed, have been specially endowed with sterility, in order to prevent their confusion. This view certainly seems at first highly probable, for species living together could hardly have been kept distinct had they been capable of freely crossing. The subject is in many ways important for us, more especially as the sterility of species when first crossed, and that of their hybrid offspring, cannot have been acquired, as I shall show, by the preservation of successive profitable degrees of sterility. It is an incidental result of differences in the reproductive systems of the parent species."

In dealing with this subject Darwin points out that we must be careful to distinguish between "the sterility of species when first crossed, and the sterility of hybrids produced from them." In the former case, the reproductive organs of each individual are in a perfectly normal condition, while hybrids appear to be generally impotent owing to some imperfection in the reproductive organs themselves. They are not perfectly fertile, as a rule, either with each other, or with either of the parent forms. 
In striking contrast to the sterility between species is the fertility of varieties. If, as Darwin believes, varieties are incipient species, we should certainly expect to find them becoming less and less fertile with other fraternal varieties, or with the parent forms in proportion as they become more different. Yet experience appears to teach exactly the opposite; but the question is not a simple one, and the results are not so conclusive as appears at first sight. Let us first see how Darwin met this obvious contradiction to his view.

In the first place, he points out that all species are not infertile when crossed with other species. The sterility of various species, when crossed, is so different in degree, and graduates away so insensibly, and the fertility of pure species is so casily affected by various circumstances, that it is most difficult to say where perfect fertility ends and sterility begins. "It can thus be shown that neither sterility nor fertility afford any certain distinction between species and varieties." Darwin cites several cases in plants in which crosses between species have been successfully accomplished. The following remarkable results are also recorded: "Individual plants in certain species of Lobelia, Verbascum, and Passiflora can easily be fertilized by pollen from a distinct species, but not by pollen from the same plant, though this pollen can be proved to be perfectly sound by fertilizing other plants or species. In the genus Hippeastrum, in Corydalis as shown by Professor Hildebrand, in various orchids as shown by Mr. Scott and Fritz. Miiller, all the individuals are in this peculiar condition. So that with some species, certain abnormal individuals, and in other species all the individuals, can actually be hybridized much more readily than they can be fertilized by pollen from the same individual plant!",

$1 \Lambda$ somewhat parallel case has recently been discovered by Castle for the hermaphroditic ascidian Ciona intestinalis. In this case the spermatozon of any individual fail to fertilize the eggs of the same individual, although they will fertilize the eggs of any wher inclividual. 
In regard to animals, Darwin concludes that " if the genera of animals are as distinct from each other as are the genera of plants, then we may infer that animals more widely distinct in the scale of nature can be crossed more easily than in the case of plants; but the hybrids themselves are, I think, more sterile.'

The most significant fact in this connection is that the more widely different two species are, so that they are placed in different families, so much the less probable is it that cross-fertilization will produce any result. From this condition of infertility there may be traced a gradation between less different forms of the same genus to almost complete, or even complete, fertility between closely similar species. Darwin further points out that: "The hybrids raised from two species which are very difficult to cross, and which rarely produce any offspring, are generally very sterile; but the parallelism between the difficulty of making a first cross, and the sterility of the hybricls thus produced - two classes of facts which are generally confounded together-is by no means strict. There are many cases, in which two pure species, as in the genus Verbascum, can be united with unusual facility, and produce numerous hybrid offspring, yet these hybrids are remarkably sterile. On the other hand, there are species which can be crossed very rarely, or with extreme difficulty, but the hybrids, when at last produced, are very fertile. Even within the limits of the same genus, for instance in Dianthus, these two opposite cases occur."

In regard to reciprocal crosses Darwin makes the following important statements: "The diversity of the result in reciprocal crosses between the same two species was long ago observed by Kölreuter. To give an instance: Mirabilis jalapa can easily be fertilized by the pollen of $M$. longiflora, and the hybrids thus produced are sufficiently fertile; but Kölreuter tried more than two hundred times, during eight 
following years, to fertilize reciprocally $M$. longiflora with the pollen of $M$. jalapa, and utterly failed."

A formal interpretation of this difference can be easily imagined. The infertility in one direction may be due to some physical difficulty met with in penetrating the stigma, or style. For instance, the tissue in one species may be too compact, or the style too long. Pfluger, who carried out a large number of experiments by cross-fertilizing different species of frogs, reached the conclusion that the spermatozoa having small and pointed heads could cross-fertilize more kinds of eggs, than could the spermatozoa with large blunt heads. This is probably due to the ability of the smaller spermatozoa to penetrate the jelly around the eggs, or the pores in the surface of the egg itself. But there are also other sides to this question, as recent results have shown, for, even if a foreign spermatozoon can enter an egg, it does not follow that the development of the egg will take place. Here the difficulty is due to some obscure processes in the egg itself. Now that we know more of the nicely balanced combinations that take place during fertilization of the egg, and during the process of cell division, we can easily see that if the processes were in the least different in the two species it might be impossible to combine them in a single act.

"Now do these complex and singular rules indicate that species have been endowed with sterility simply to prevent their becoming confounded in nature? I think not. For why should the sterility be so extremely different in degree, when various species are crossed, all of which we must suppose it would be equally important to keep from blending together?"

"The foregoing rules and facts, on the other hand, appear to me clearly to indicate that the sterility both of first crosses and of hybrids is simply incidental or dependent on unknown differences in their reproductive systems; the differences being of so peculiar and limited a nature, that, in reciprocal 
crosses between the same two species, the male sexual element of the one will often freely act on the female sexual element of the other, but not in a reversed direction."

Does Darwin give here a satisfactory answer to the difficulty that he started out to explain away? On the whole, the reader will admit, I think, that he has fairly met the situation, in so far as he has shown that there is no absolute line of demarcation between the power of intercrossing of varieties and races, and of species. It is also cxtremely important to have found that the difficultics increase, so to spack, even beyond the limits of the spccics; since species, belonging to different genera, are as a rule more difficult to intercross than when they belong to the same genus. The further question, as to whether there are differences in respect to the power of intercrossing between different kinds of varieties, such as those dependent on selection of fluctuating variations, of local conditions, of mutations, etc., is far from being settled at the present time.

That this property of species is useful to them, in the somewhat unusual sense that it keeps them from freely mingling with other species, is true; but, as has been said, this would be a rather peculiar kind of adaptation. If, however, it be claimed that this property is useful to species, as Darwin himself claims, then, as he also points out, it is a useful acquirement that cannot have arisen through natural selection. It is not difficult to show why this must be so. If two varieties were to some extent at the start less fertile, inter sc, than with their own kind, the only way in which they could become more infertile through selection would be by selecting those individuals in each generation that are still more infertile, but the forms of this sort would, ex hypothese, become less numerous than the descendants of each species itself, which would, therefore, supplant the less fertile ones.

Darwin's own statement in regard to this point is as follows :- 
"At one time it appeared to me probable, as it has to others, that the sterility of first crosses and of hybrids might have been slowly acquired through the natural selection of slightly lessened degrees of fertility, which, like any other variation, spontaneously appeared in certain individuals of one variety when crossed with those of another variety. For it would clearly be advantageous to two varieties or incipient species, if they could be kept from blending, on the same principle that, when man is selecting at the same time two varieties, it is necessary that he should keep them separate.

"In considering the probability of natural selection having come into action, in rendering species mutually sterile, the greatest difficulty will be found to lie in the existence of many graduated steps from slightly lessened fertility to absolute sterility. It may be admitted that it would profit an incipient species, if it were rendered in some slight degree sterile when crossed with its parent form or with some other variety; for thus fewer bastardized and deteriorated offspring would be produced to commingle their blood with the new species in process of formation. But he who will take the trouble to reflect on the steps by which this first degree of sterility could be increased through natural selection to that high degree which is common with so many species, and which is universal with species which have been differentiated to a generic or family rank, will find the subject extratordinarily complex. After mature reflection it seems to me that this could not have been effected through natural selection. Take the case of any two species which, when crossed, produced few and sterile offspring; now, what is there which could favor the survival of those individuals which happened to be endowed in a slightly higher degree with mutual infertility, and which thus approached by one small step toward absolute sterility? Yet an advance of this kind, if the theory of natural selection be brought to bear, must have inces- 
santly occurred with many species, for a multitude are mutually quite barren."

Darwin points out the interesting parallel existing between the results of intercrossing, and those of grafting together parts of different species.

"As the capacity of one plant to be grafted or budded on another is unimportant for their welfare in a state of nature, I presume that no one will suppose that this capacity is a specially endowed quality, but will admit that it is incidental on differences in the laws of growth of the two plants. We can sometimes see the reason why one tree will not take on another, from differences in their rate of growth, in the hardness of their wood, in the period of the flow or nature of their sap, etc.; but in a multitude of cases we can assign no reason whatever. Great diversity in the size of two plants, one being woody and the other herbaceous, one being evergreen and the other deciduous, and adapted to widely different climates, do not always prevent the two grafting together. As in hybridization, so with grafting, the capacity is limited by systematic affinity, for no one has been able to graft together trees belonging to quite distinct families; and, on the other hand, closely allied species, and varieties of the same species, can usually, but not invariably, be grafted with ease. But this capacity, as in hybridization, is by no means absolutely governed by systematic affinity. Although many distinct genera within the same family have been grafted together, in other cases species of the same genus will not take on each other. The pear can be grafted far more readily on the quince, which is ranked as a distant genus, than on the apple, which is a member of the same genus. Even different varieties of the pear take with different degrees of facility on the quince; so do different varieties of the apricot and peach on certain varieties of the plum."

"We thus see, that although there is a clear and great difference between the mere adhesion of grafted stocks, and 
the union of the male and female elements in the act of reproduction, yet that there is a rude degree of parallelism in the results of grafting and of crossing of distinct species. And we must look at the curious and complex laws governing the facility with which trees can be grafted on each other as inciclental on unknown differences in their vegetative systems, so I believe that the still more complex laws governing the facility of first crosses are incidental on unknown differences in their reproductive systems. . . The facts by no means seem to indicate that the greater or lesser difficulty of either grafting or crossing various species has been a special endowment; although in the case of crossing, the difficulty is as important for the endurance and stability of specific forms, as in the case of grafting it is unimportant for their welfare."

\section{Weismann's Germinal Selection}

We cannot do better, in bringing this long criticism of the Darwinian theory to an end, than by considering the way in which Weismann has attempted in his paper on "Germinal Selection" to solve one of the "patent contradictions" of the selection theory. He calls attention, in cloing so, to what he regards as a vital weakness of the theory in the form in which it was left by Darwin himself. Weismann says:-

"The basal idea of the essay - the existence of Germinal Selection - was propounded by me some time since, ${ }^{1}$ but it is here for the first time fully set forth and tentatively shown to be the necessary complement of the process of selection. Knowing this factor, we remove, it seems to me, the patent contradiction of the assumption that the general fitness of organisms, or the adaptations necssary to their existence, are produced by accidental variations - a contradiction which

${ }^{1}$ Neue Gedanken zur Vererbungsfrage, eine Antwort an Herbert Spencer, Jena, I 895 . 
formed a serious stumbling-block to the theory of selection. Though still assuming that the primary variations are 'accidental,' I yet hope to have demonstrated that an interior mechanism exists which compels them to go on increasing in a definite direction, the moment selection intervenes. Definitcly dircted variation cxists, but not predestined variation, running on independently of the life conditions of the organism, as Nägeli, to mention the most extreme advocate of this doctrine, has assumed; on the contrary, the variation is such as is elicited and controlled by those conditions themselves, though indirectly."

"The real aim of the present essay is to rehabilitate the principle of selection. If I should succeed in reinstating this principle in its emperilled rights, it would be a source of extreme satisfaction to me; for I am so thoroughly convinced of its indispensability as to believe that its demolition would be synonymous with the renunciation of all inquiry concerning the causal relation of vital phenomena. If we could understand the adaptations of nature, whose number is infinite, only upon the assumption of a teleological principle, then, I think, there would be little inducement to trouble ourselves about the causal connection of the stages of ontogenesis, for no good reason would exist for excluding teleological principles from this field. Their introduction, however, is the ruin of science." 1

Weismann states that those critics who maintain that selection cannot create, but only reject, "fail to see that precisely through this rejection its creative efficacy is asserted." There is raised here, though not for the first time, a point that is of no small importance for both Darwinians and antiDarwinians to consider; for, without further examination, it is by no mear: self-evident, as Weismann implies, that by exterminating all variations that are below the average the

1 Translated by J. McCormack. The Open Court Publishing Company. The following quotations are also taken from this translation. 
standard of successive generations could ever be raised beyond the most extreme fluctuating variation. At least this appears to be the case if individual, fluctuating variations be the sort selected, and it is to this kind of variation to which Weismann presumably refers. Without discussing this point here, let us examine further what Weismann has to say. He thinks that while in each form there may be a very large number of possible variations, yet there are also impossible variations as well, which do not appear. "The cogency, the irresistible cogency as I take it, of the principle of selection is precisely its capacity of explaining why fit structures always arise, and this certainly is the great problem of life." Weismann points out that it is a remarkable fact that to-day, after science has been in possession of this principle for something over thirty years, "during which time she has busily occupied herself with its scope, the estimation in which the theory is held should be on the decline." "It would be easy to enumerate a long list of living writers who assign to it a subordinate part only in evolution, or none at all." "Even Huxley implicitly, yet distinctly, intimated a doubt regarding the principle of selection when he said: 'Even if the Darwinian hypothesis were swept away, evolution would still stand where it is.' Therefore he, too, regarded it as not impossible that this hypothesis should disappear from among the great explanatory principles by which we seek to approach nearer to the secrets of nature."

Weismann is not, however, of this opinion, and believes that the present depression is only transient, because it is only a reaction against a theory that had been exalted to the highest pinnacle. He thinks that the principle of selection is not overestimated, but that naturalists imagined too quickly that they understood its workings. "On the contrary, the deeper they penetrated into its workings the clearer it appeared that something was lacking, that the action of the principle, though upon the whole clear and representable, yet 
when carefully looked into encountered numerous difficulties, which were formidable, for the reason that we were unsuccessful in tracing out the actual details of the individual process, and, therefore, in fixing the phenomenon as it actually occurred. We can state in no single case how great a variation must be to have selective value, nor how frequently it must occur to acquire stability. We do not know when and whether a desired useful variation really occurs, nor on what its appearance depends; and we have no means of ascertaining the space of time required for the fulfilment of the selective processes of nature, and hence cannot calculate the exact number of such processes that do and can take place at the same time in the same species. Yet all this is necessary if we wish to follow out the precise details of a given case.

"But perhaps the most discouraging circumstance of all is, that we can assert in scarcely a single actual instance in nature whether an observed variation is useful or not -a drawback that I distinctly emphasized some time ago. Nor is there much hope of betterment in this respect, for think how impossible it would be for us to observe all the indivicluals of a species in all their acts of life, be their habitat ever so limited - and to observe all this with a precision enabling us to say that this or that variation possessed selective value, that is, was a decisive factor in determining the existence of the species."

"And thus it is everywhere. Even in the most indubitable cases of adaptation as, for instance, in that of the striking protective coloring of many butterflies, the sole ground of inference that the species on the whole is adequately adapted to its conditions of life, is the simple fact that the species is, to all appearances, preserved undiminished, but the inference is not at all permissible that just this protective coloring has selective value for the species, that is, if it were lacking, the species would necessarily have perished."

Few opponents of Darwinism could give a more pessimist " 
account of the accomplishments of the theory of natural selection than this, by one of the leaders of the modern school: "Discouraging, therefore, as it may be that the control of nature in her minutest details is here gainsaid us, yet it were equivalent to sacrificing the gold to the dross, if simply from our inability to follow out the details of the individual case we should renounce altogether the principle of selection, or should proclaim it as only subsidiary, on the ground that we believe the protective coloring of the butterfly is not a protective coloring, but a combination of colors inevitably resulting from internal causes. The protective coloring remains a protective coloring whether at the time in question it is or is not necessary for the species; and it arose as protective coloring - arose not because it was a constitutional necessity of the animal's organism that here a red and there a white, black, or yellow spot should be produced, but because it was advantageous, because it was necessary for the animal. There is only one explanation possible for such patent adaptations, and that is selection. What is more, no other natural way of their originating is conceivable, for we have no right to assume teleological forces in the domain of natural phenomena."

Weismann states that he does not accept Eimers's view that the markings of the wings of the butterflies of the genus Papilio are due to a process of evolution in a direct line, independent of external causes.

"On the contrary, I believe it can be clearly proved that the wing of the butterfly is a tablet on which Nature has inscribed everything she has deemed advantageous to the preservation and welfare of her creatures, and nothing else; or, to abandon the simile, that these color patterns have not proceeded from inward evolutional forces but are the result of selection. At least in all places where we do understand their biological significance these patterns are constituted and distributed over the wing exactly as utility would require." 


\section{Darwin's Artificial and Natural Selection I 59}

Again: "I should be far from maintaining that the markings arose unconformably to law. Here, as elsewhere, the dominance of law is certain. But I take it, that the laws involved, that is, the physiological conditions of the variation, here are without exception subservient to the ends of a higher power - utility; and that it is utility primarily that determines the kind of colors, spots, streaks, and bands that shall originate, as also their place and mode of disposition. The laws come into consideration only to the extent of conditioning the quality of the constructive materials - the variations, out of which selection fashions the designs in question. And this also is subject to important restrictions, as will appear in the sequel." This conclusion contains all that the most ardent Darwinian could ask.

He rejects the idea that internal laws alone could have produced the result, because :-

"If internal laws controlled the markings on butterflies' wings, we should expect that some general rule could be established, requiring that the upper and under surfaces of the wings should be alike or that they should be different, or that the fore wings should be colored the same as or differently from the hind wings, etc. But in reality all possible kinds of combinations occur simultaneously, and no rule holds throughout. Or, it might be supposed that bright colors should occur only on the upper surface or only on the under surface, or on the fore wings or only on the hind wings. But the fact is they occur indiscriminately, now here, now there, and no one method of appearance is uniform throughout all the species. But the fitness of the various distributions of colors is apparent, and the moment we apply the principle of utility we know why in the diurnal butterflies the upper surface alone is usually variegated and the under surface protectively colored, or why in the nocturnal butterflies the fore wings have the appearance of bark, of old wood, or of a leaf, whilst the hind wings, which are covered when resting, alone 
are brilliantly colored. On this theory we also understand the exceptions to these rules. We comprehend why Danaids, Heliconids, Euploids, and Acracids, in fact all diurnal butterflies offensive to the taste and smell, are mostly brightly marked and equally so on both surfaces, whilst all species not thus exempt from persecution have the protective coloring on the under surface and are frequently quite differently colored there from what they are on the upper.

"In any event, the supposed formative laws are not obligatory. Dispensations from them can be issued and are issued whencier utility requires it."

Dispensations from the laws of growth! Does not a philosophy of this sort scem to carry us back into the dark ages? Is this the best that the Darwinian school can do to protect itself against the difficulties into which its chief disciple confesses it has fallen?

Weismann lays great emphasis on the case of the Indian leaf-butterfly, Kallima inachis; and points out that the leaf markings are executed "in absolute independence of the other uniformities governing the wing."

"The venation of the wing is utterly ignored by the leaf markings, and its surface is treated as a tabula rasa upon which anything conceivable can be drawn. In other words, we are presented here with a bilatcrally symmetrical figure engraved on a surface which is essentially radially symmetrical in its divisions.

"I lay unusual stress upon this point because it shows that we are dealing here with one of those cases which cannot be explained by mechanical, that is, by natural means, unless natural selection actually exists and is actually competent to create new properties; for the Lamarckian principle is excluded here ab initio, seeing that we are dealing with a formation which is only passive in its effects: the leaf markings are effectual simply by their existence and not by any function which they perform; they are present in flight as well 


\section{Darwin's Artificial and Natural Selection I6I}

as at rest, during the absence of a danger, as well as during the approach of an enemy.

"Nor are we helped here by the assumption of purely internal motive forces, which Nägeli, Askenasy, and others have put forward as supplying a mechanical force of evolution. It is impossible to regard the coincidence of an Indian butterfly with the leaf of a tree now growing in an Indian forest as fortuitous, as a lusus nature. Assuming this seemingly mechanical force, therefore, we should be led back inevitably to a teleological principle which produces adaptive characters and which must have deposited the directive principle in the very first germ of terrestrial organisms, so that after untold ages at a definite time and place the illusive leaf markings should be developed. The assumption of preëstablished harmony between the evolution of the ancestral line of the tree with its prefigurative leaf, and that of the butterfly with its imitating wing, is absolutely necessary here, as I pointed out many years ago, but as is constantly forgotten by the promulgators of the theory of internal evolutionary forces."

Weismann concludes, therefore, that for his present purpose it suffices to show "that cases exist wherein all natural explanations except that of selection fail us," and' he then proceeds to point out that even the natural selection of Darwin and of Wallace also fail to give us a reasonable explanation of how, for example, the markings on the wings of the Kallima butterfly have come about. The main reason that he gives to show that this is the case rests on the difficulty of the assumption that the right variations should always be present in the right place. Here "is the insurmountable barrier for the explanatory power of the principle [natural selection] for who, or what, is to be our guarantee that the dark scales shall appear at the exact spots on the wing where the midrib of the leaf must grow? And that later dark scales shall appear at the exact spots to which the midrib must be prolonged? And that still later such dark scales 
shall appear at the places whence the lateral ribs start, and that here also a definite acute angle shall be preserved." Thus the philosopher in his closet multiplies and magnifies the difficulties for which he is about to offer a panacea. Had the same amount of labor been spent in testing whether the life of this butterfly is so closely dependent on the exact imitation of the leaf, we might have been spared the pains of this elaborate exordium. There are at least some grounds for suspicion that the whole case of Kallima is "made up." If this should prove true, it will be a bad day for the Darwinians, unless they fall back on Weismann's statement that their theory is insufficient to prove a single case!

Weismann has used Kallima only as the most instructive illustration. The objections that are here evident are found not only in the cases of protective coloration, but " are applicable in all cases where the process of selection is concerned. Takc, for example, the case of instincts that are called into action only once in life, as the pupal performances of insects, the fabrication of cocoons, etc. How is it that the useful variations were always present here?" Weismann concludes that "something is still wanting to the selection theory of Darwin and Wallace, which it is obligatory on us to discover, if we possibly can, and without which selection as yet offers no complete explanation of the phyletic processes of transformation." Weismann's first step in the solution of the difficulty is contained in the following statement:-

"My inference is a very simple one: if we are forced by the facts on all hands to the assumption that the useful variations which render selection possible are always present, then, some frofound conncction must cxist betwech the utility of a aration and its actual appearance, or, in other words, the direction of the variation of a fart must be determined by utility, and we shall have to see whether facts exist that confirm our conjecture."

Weismann finds the solution in the method by which the 


\section{Darwin's Artificial and Natural Selection ${ }^{6} 6_{3}$}

breeder has obtained his results in artificial selection. For instance, the long-tailed variety of the domestic cock of Japan owes its existence, it is claimed, to skilful selection, and not at all to the circumstance that, at some period of the race's history, a cock with tail-feathers six feet in length suddenly and spasmodically appeared.

Weismann continues: "Now what does this mean? Simply that the hereditary diathesis, the germinal constitution (the Anlage) of the breed was changed in the respect in question, and our conclusion from this and numerous similar facts of artificial selection runs as follows: by the selection alone of the plus or minus variations of a character is the constant modification of that character in the plus or minus direction detcrmined. Obviously the hereditary diminution of a part is also effected by the simple selection of the individuals in each generation possessing the smallest parts, as is proved, for example, by the tiny bills and feet of numerous breeds of doves. We may assert, therefore, in general terms: a definitely directed progressive variation of a given part is produced by continued selection in that definite direction. This is no hypothesis, but a direct inference from the facts and may also be expressed as follows: by a selection of the kind referred to the germ is progressively modified in a manner corresponding with the production of a definitely dirccted progressive variation of the part."

So far there is nothing essentially new offered, since Darwin often tacitly recognized that the standard of variation could be raised in this way, and in some places he has made definite statements that this will take place. Weismann thinks that after each selection, fluctuation will then occur around a higher average (mode). He says "that this is a fact," and is proved by the case of the Japanese cock. It need scarcely be pointed out that it is an assumption, based on what is supposed to have taken place in this bird, and is not a "fact." 
Weismann continues: "But the question remains, why is this the fact?" He believes his hypothesis of the existence of determinants in the germ gives a satisfactory answer to this "why." "According to this theory every independent and hereditarily variable part is represented in the germ by a determinant, whose size and power of assimilation corresponds to the size and vigor of the part. These determinants multiply as do all vital units by growth and division, and necessarily they increase rapidly in every individual, and the more rapidly the greater the quantity of the germinal cells the individual produces. And since there is no more reason for excluding irregularities of passive nutrition, and of the supply of nutriment in these minute, microscopically invisible parts, than there is in the larger visible parts of the cells, tissues, and organs, consequently the descendants of a determinant can never all be exactly alike in size and capacity of assimilation, but they will oscillate in this respect to and fro about the maternal determinant as about their zero point, and will be partly greater, partly smaller, and partly of the same size as that. In these oscillations, now, the material for further selection is presented, and in the inevitable fluctuations of the nutrient supply, I see the reason why every step attained immediately becomes the zero point of new fluctuations, and consequently why the size of a part can be augmented or diminished by selection without limit, solely by the displacement of the zero point of variation as the result of selection."

The best illustration of this process of germinal selection is found, Weismann believes, in the case of the degeneration of organs. "For in most retrogressive processes actia't selection in Darwin's sense plays no part, and advocates of the Lamarckian principle, as above remarked, have rightly denied that active selection, that is, the selection of individuals possessing the useless organ in its most reduced state, is sufficient to explain the process of degeneration. I, 


\section{Darwin's Artificial and Natural Sclection 165}

for my part, have never assumed this, and have on this very account enunciated the principle of pammixia. Now, although this, as I have still no reason for doubting, is a perfectly correct principle, which really does have an essential and inclispensable share in the process of retrogression, still it is not alone sufficient for a full explanation of the phenomena. My opponents, in advancing this objection, were right, to the extent indicated, and as I expressly acknowledge, although they were unable to substitute anything positive in its stead or to render my explanation complete. The very fact of the cessation of control over the organ is sufficient to explain its degencration, that is, its deterioration, the disharmony of its parts, but not the fact which actually and always occurs where an organ has become useless - viz., its gradual and uncasing diminution continuing for thonsands ana thousands of years and culminating in its final and absolute effacement."

If then neither selection of persons nor the cessation of personal selection can explain the phenomenon, we must look elsewhere for the answer. This Weismann finds in the application of Roux's hypothesis of the struggle of the parts to obtain nourishment.

"The production of the long tail-feathers of the Japanese cock does not repose solely on the displacement directly effected by personal selection, of the zero point of variation upward, but that it is also fostered and strensthened by scrminal sclction. Were that not so, the phenomena of the transmutation of species, in so far as fresh growth and the enlargement and complication of organs already present are concerned, would not be a whit more intelligible than they were before."

Thus Weismann has piled up one hypothesis on another as though he could save the integrity of the theory of natural selection by adding new speculative matter to it. The most unfortunate feature is that the new speculation is skilfully removed from the field of verification, and invisible germs 
whose sole functions are those which Weismann's imagination bestows on them, are brought forward as though they could supply the deficiencies of Darwin's theory. This is, indeed, the old method of the philosophizers of nature. An imaginary system has been invented which attempts to explain all difficulties, and if it fails, then new inventions are to be thought of. Thus we see where the theory of the selection of fluctuating germs has led one of the most widely known disciples of the Darwinian theory.

The worst feature of the situation is not so much that Weismann has advanced new hypotheses unsupported by experimental evidence, but that the speculation is of such a kind that it is, from its very nature, unverifiable, and therefore useless. Weismann is mistaken when he assumes that many zoologists object to his methods because they are largely speculative. The real reason is that the speculation is so often of a kind that cannot be tested by observation or by experiment. 


\section{CHAPTER VI}

\section{DARWIN'S THEORY OF SEXUAL SELECTION}

\section{Sexual Selection}

THE theory of sexual selection was formulated by Darwin, even in the first edition of the "Origin of Species," but was developed at much greater length in "The Descent of Man." "This form of selection depends, not on a struggle for existence in relation to other organic beings or to external conditions, but on a struggle between the individuals of one sex, generally the males, for the possession of the other sex. The result is not death to the unsuccessful competitor, but few or no. offspring. Sexual selection is, therefore, less rigorous than natural selection. Generally the most vigorous males, those which are best fitted for their place in nature, will leave most progeny. But in many cases victory depends, not so much on general vigor, as on having special weapons, confined to the male sex. A hornless stag or spurless cock would have a poor chance of leaving numerous offspring. Sexual selection, by always allowing the victor to breed, might surely give indomitable courage, length to the spur, and strength to the wing to strike in the spurred leg in nearly the same manner as the brutal cock-fighter by the careful selection of his best cocks." It is important to notice that the theory of sexual selection is admittedly an extension of the selection principle into a new field. Having accounted for domesticated animals and plants by artificial selection, and for the adaptations of wild species by natural selection, there remained only to account for the second- 
ary sexual differences between the sexes by the principle of sexual selection.

There are two ways in which Darwin supposes sexual selection to act: (I) through competition of the individuals of the same sex with each other, - the strongest or best-equipped for fighting or for fincling the individuals of the other sex gaining an advantage; (2) through selection by the individuals of one sex of certain preferred individuals of the other sex.

The first category is natural selection applied to the members of one sex in competition with each other, although the result does not lead to the death of the unsuccessful individual, but excludes it from leaving progeny. In the second category a new element is introduced, namely, the sclective power of the individuals of one sex, usually the female. It is this part that adds a distinctly new element to Darwin's other two theories of selection, and it is this part that we naturally think of as the theory of sexual selection par cxcellence. Darwin makes, however, no sharp distinction between these two sides of his theory, but includes both under the heading of sexual selection.

In order to get the theory itself before us in as concrete form as possible, let us examine some of the cases that Darwin has given to show how he supposes the process to be carried out.

"There are many other structures and instincts which must have been devcloped through sexual selection - such as the weapons of offence and the means of defence of the males for fighting with and driving away their rivals - their courage and pugnacity - their various ornaments - their contrivances for producing vocal or instrumental music - and their glands for emitting odors, most of these latter structures serving oniy to allure or excite the female. It is clear that these characters are the result of sexual and not of ordinary selection, since unarmed, unornamented, or unattractive males would succeed equally well in the battle for life and in leaving 
a numerous progeny, but for the presence of better-endowed males. We may infer that this would be the case, because the females, which are unarmed and unornamented, are able to survive and procreate their kind. Secondary sexual characters of the kind just referred to will be fully discussed in the following chapters, as being in many respects interesting, but especially as depending on the will, choice, and rivalry of the individuals of either sex. When we behold two males fighting for the possession of the female, or several male birds displaying their gorgeous plumage, and performing strange antics before an assembled body of females, we cannot doubt that, though led by instinct, they know what they are about, and consciously exert their mental and bodily powers."

This general statement gives an idea of the class of phenomena that Darwin proposes to explain by the theory of sexual selection. The close resemblance between this process and that of artificial selection may be gathered from the following statement :-

"Just as man can improve the breed of his game-cocks by the selection of those birds which are victorious in the cockpit, so it appears that the strongest and most vigorous males, or those provided with the best weapons, have prevailed under nature, and have led to the improvement of the natural breed or species. A slight degree of variability leading to some advantage, however slight, in reiterated deadly contests would suffice for the work of sexual selection ; and it is certain that secondary sexual characters are eminently variable. Just as man can give beauty, according to his standard of taste, to his male poultry, or more strictly can modify the beauty originally acquired by the parent species, can give to the Sebright bantam a new and elegant plumage, an erect and peculiar carriage - so it appears that female bircls in a state of nature have, by a long selection of the more attractive males, added to their beauty or other attractive qualities. No doubt this implies powers of discrimination and taste on the part of the female 
which will at first appear extremely improbable; but by the facts to be adduced hereafter, I hope to be able to show that the females actually have these powers. When, however, it is said that the lower animals have a sense of beauty, it must not be supposed that such sense is comparable with that of a cultivated man, with his multiform and complex associated icleas. A more just comparison would be between the taste for the beautiful in animals, and that in the lowest savages, who admire and deck themselves with any brilliant, glittering, or curious object."

Darwin did not close his eyes to the difficulties which the theory had to contend against. One of the most formidable of these objections is described in the following words: "Our difficulty in regard to sexual selection lies in understanding how it is that the males which conquer other males, or those which prove the most attractive to the females, leave a greater number of offspring to inherit their superiority than their beaten and less attractive rivals. Unless this result does follow, the characters which give to certain males an advantage over others could not be perfected and augmented through sexual selection. When the sexes exist in exactly equal numbers, the worst-endowed males will (except where polygamy prevails) ultimately find females, and leave as many offspring, as well fitted for their general habits of life, as the best-endowed males. From various facts and considerations, I formerly inferred that with most animals, in which secondary sexual characters are well developed, the males considerably exceeded the females in number; but this is not by any means always true. If the males were to the females as two to one, or as three to two, or even in a somewhat lower ratio, the whole affair would be simple; for the better-armed or more attractive males would leave the largest number of offspring. But after investigating, as far as possible, the numerical proportion of the sexes, I do not believe that any great inequality in number 
commonly exists. In most cases sexual selection appears to have been effective in the following manner.

"Let us take any species, a bird for instance, and divide the females inhabiting a district into two equal bodies, the one consisting of the more vigorous and better-nourished individuals, and the other of the less vigorous and healthy. The former, there can be little doubt, would be ready to breed in the spring before the others; and this is the opinion of Mr. Jenner Weir, who has carefully attended to the habits of birds during many years. There can also be no doubt that the most vigorous, best-nourished and earliest breeders would on an average succeed in rearing the largest number of fine offspring. The males, as we have seen, are generally ready to breed before the females; the strongest, and with some species the best-armed of the males, drive away the weaker; and the former would then unite with the more vigorous and better-nourished females, because they are the first to breed. Such vigorous pairs would surely rear a larger number of offspring than the retarded females, which would be compelled to unite with the conquered and less powerful males, supposing the sexes to be numerically equal; and this is all that is wanted to add, in the course of successive generations, to the size, strength and courage of the males, or to improve their weapons." .

I shall comment later on the points here raised, but we should not let this opportunity pass without noticing, that even if the pairing were to follow according to the method here imagined, still the argument breaks down at the critical point, for there is no evidence that the more precocious females would rear a larger number of offspring than the more normal females, or even those that breed somewhat later.

The greater eagerness of the males which has been observed in so many different classes of animals is accounted for as follows :- 
"But it is difficult to understand why the males of species, of which the progenitors were primordially free, should invariably have acquired the habit of approaching the females, instead of being approached by them. But in all cases, in order that the males should seek efficiently, it would be necessary that they should be endowed with strong passions; and the acquirement of such passions would naturally follow from the more eager leaving a larger number of offspring than the less eager."

Thus we are led to the rather complex conclusion, that the more eager males will leave more descendants, and those that are better endowed with ornaments will be the ones selected. But unless it can be shown that there is some connection between greater eagerness and better ornamentation, it might often occur that the less ornamented were the more eager individuals, in which case there would be an apparent conflict between the two acquirements.

After giving some cases of the greater variability of the males, in respect to characters that are not connected with sexual selection, and presumably not the result of any kind of selection, Darwin concludes: "Through the action of sexual and natural selection male animals have been rendered in very many instances widely different from their females; but independently of selection the two scxes, from differing constitutionally, tend to vary in a somewhat different manner. The female has to expend much organic matter in the formation of her ova, whereas the male expends much force in fierce contests with his rivals, in wandering about in search of the female, in exerting his voice, pouring out odoriferous secretions, etc. : and this expenditure is generally concentrated within a short period. The great vigor of the male during the season of love secms often to intensify his colors, independently of any marked difference from the female. In mankind, and even as low down in the organic scale as in the Lepidoptera, the temperature of the 


\section{Darwin's Theory of Sexual Selection}

body is higher in the male than in the female, accompanied in the case of man by a slower pulse. On the whole, the expenditure of matter and force by the two sexes is probably nearly equal, though effected in very different ways and at different rates."

Again: "From the causes just specified, the two sexes can hardly fail to differ somewhat in constitution, at least during the breeding season; and although they may be subjected to exactly the same conditions, they will tend to vary in a different manner. If such variations are of no service to either sex, they will not be accumulated and increased by sexual or natural selection. Nevertheless, they may become permanent if the exciting cause acts permanently; and in accordance with a frequent form of inheritance they may be transmitted to that sex alone in which they first appeared. In this case, the two sexes will come to present permanent, yet unimportant, differences of character. For instance, Mr. Allen shows that with a large number of birds inhabiting the northern and southern United States, the specimens from the south are darker-colored than those from the north; and this seems to be the direct result of the difference in temperature, light, etc., between the two regions. Now, in some few cases, the two sexes of the same species appear to have been differently affected; in the Agcleus phanicus the males have had their colors greatly intensified in the south; whereas with Cardinalis virginianus it is the females which have been thus affected: with Quiscalus major the females have been rendered extremely variable in tint, whilst the males remain nearly uniform."

The admissions contained in this statement would seem to jeopardize the entire question, for, if it is admitted that, on account of the difference in the constitution of the two sexes, the influence of the surrounding conditions would produce a different effect on them, it would seem that there is no need whatsoever for the theory of sexual selection. What Darwin 
is probably attempting to show is that the material for the further action of sexual selection is already given; but the question may well be asked, if the external conditions have done so much, why may they not have gone farther and produced the entire result?

Darwin makes the following suggestion to account for those cases in which the female is the more highly colored :-

"A few exceptional cases occur in various classes of animals, in which the females instead of the males have acquired well-pronounced secondary sexual characters, such as brighter colors, greater size, strength, or pugnacity. With birds there has sometimes been a complete transposition of the ordinary characters proper to each sex; the females having become the more eager in courtship, the males remaining comparatively passive, but apparently selecting the more attractive females, as we may infer from the results. Certain hen birds have thus been rendered more highly colored or otherwise ornamented, as well as more powerful and pugnacious than the cocks; these characters being transmitted to the female offspring alone."

Then follows immediately the discussion as to whether a double process of sexual selection may not be supposed to go on at the same time. "It may be suggested that in some cases a double process of selection has been carried on; that the males have selected the more attractive females, and the latter the more attractive males. This process, however, though it might lead to the modification of both sexes, would not make the one sex different from the other, unless indeed their tastes for the beautiful differed; but this is a supposition too improbable to be worth considering in the case of any animal, excepting man. There are, however, many animals in which the sexes resemble each other, both being furnished with the same ornaments, which analogy would lead us to attribute to the agency of sexual selection. In such cases it may be suggested with more plausibility, that there has 


\section{Darwin's Theory of Sexual Selection}

been a double or mutual process of sexual selection; the more vigorous and precocious females selecting the more attractive and vigorous males, the latter rejecting all except the more attractive females. But from what we know of the habits of animals, this view is hardly probable, for the male is generally eager to pair with any female. It is more probable that the ornaments common to both sexes were acquired by one sex, generally the male, and then transmitted to the offspring of both sexes. If, indeed, during a lengthened period the males of any species were greatly to exceed the females in number, and then during another lengthened period, but under different conditions, the reverse were to occur, a double but not simultaneous process of sexual selection might easily be carried on, by which the two sexes might be renclered widely different."

The improbability of such a process is so manifest that the suggestion can scarcely be looked upon as anything more than pure speculation. We shall have occasion later to return to the same subject, and point out its bearing more explicitly.

Nearly the whole animal kingdom is passed in review by Darwin from the point of view of the sexual selection theory. There is brought together a large number of extremely interesting facts, and if the theory did no more than merely hold them together, it has served, in this respect, a useful end. We may select some of the most instructive cases by way of illustrating the theory.

In many of the lower animals in which the sexes are separated, and these alone, of course, can be supposed to come within the range of the theory, there are no striking differences between the sexes, in regard to ornamentation, although in other respects differences may exist.

"Moreover it is almost certain that these animals have too imperfect senses and much too low mental powers, to appreciate each other's beauty or other attractions, or to feel rivalry. 
"Hence in these classes or subkingdoms, such as the Protozoa, Coelenterata, Echinodermata, Scolecida, secondary sexual characters, of the kind which we have to consider, do not occur; and this fact agrees with the belief that such characters in the higher classes have been acquired through sexual selection, which depends on the will, desire, and choice of either sex."

There are some cases, however, in which animals low in the scale show a difference in the ornamentation of the two sexes. A few cases have been recorded in the roundworms, where different shades of the same tint distinguish the sexes. In the annelids the sexes are sometimes so different, that, as Darwin remarks, they have been placed in different genera and even families, "yet the differences do not seem to be of the kind which can be safely attributed to sexual selection." In regard to the nemertian worms, although they "vie in variety and beauty of coloring with any other group in the invertebrate series," yet McIntosh states that he "cannot discover that these colors are of any service." In the copepods, belonging to the group of lower Crustacea, Darwin excludes those cases in which the males alone "are furnished with perfect swimming legs, antemma, and sense organs; the females being destitute of these organs, with their bodies often consisting of a mere distorted mass," because these extraordinary differences between the two sexes are no doubt related to their widely different habits of life. Nevertheless, it is important to observe that such extreme differences may exist in cases where sexual selection cannot come in, because of the absence of eyes in the female.

In regard to another copepod, Saphirina, Darwin points out that the males are furnished with minute scales, which exhibit beautiful changing colors, and these are absent in the females; yet he states that it would be extremely rash to conclude that these curious organs serve to attract the females. Differences in the scxes are also found in one 
species of Squilla, and a species of Gelasimus. In the latter case Darwin thinks that the difference is probably due to sexual selection. In addition to these cases, recorded by Darwin, there may be added the two remarkable cases, shown in our Figure $2 \mathrm{~A}, \mathrm{~B}$, of Calocalanus pavo, the female of

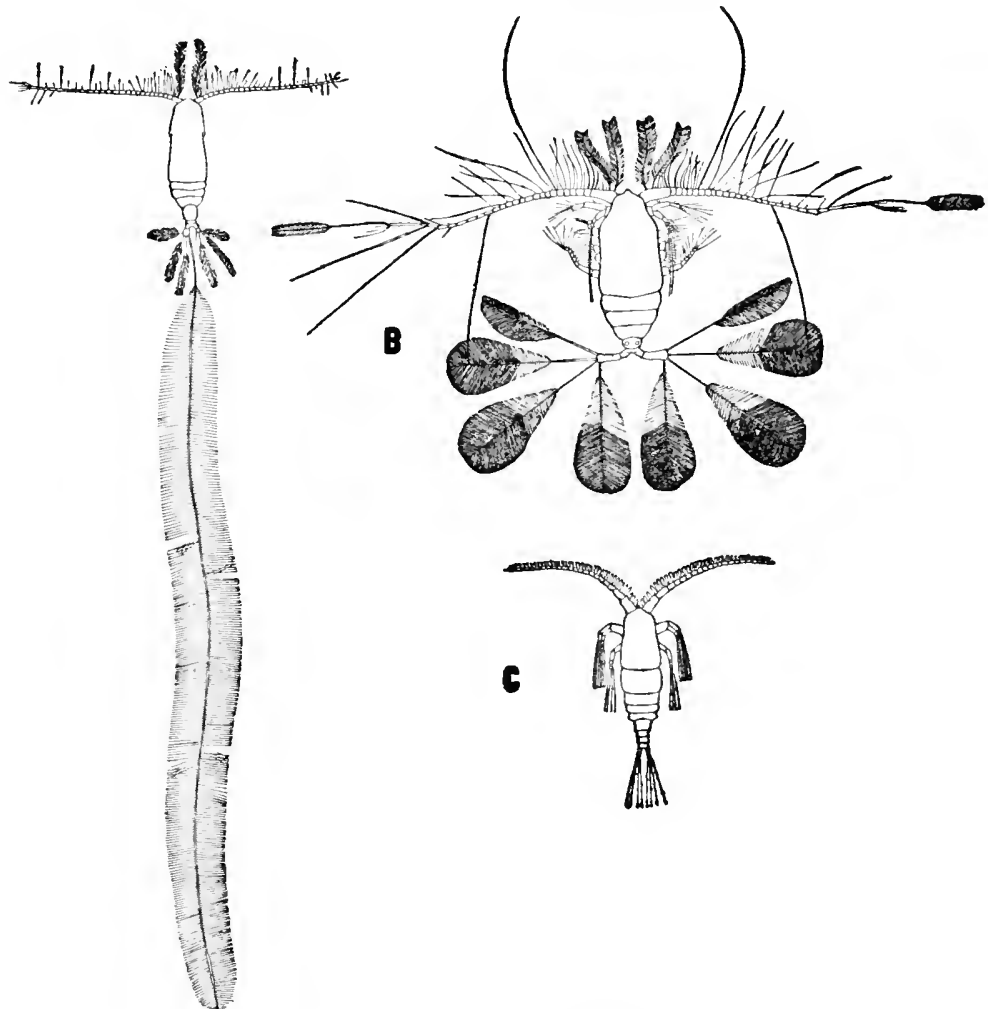

FIG. 2. - A male of the copepod, Calocalanus piumulosus. B and C, a male and a female of Calocalanus pazo. (After Giesbrecht.)

which has a gorgeous tail worthy of a peacock, and of Calocalcmus plumulosus, in which one of the setre of the tail is drawn out into a long featherlike structure. In the former, the male is much more modestly adorned, as shown in Figure $2 \mathrm{C}$; in the latter species the male is unknown.

In spiders, where as a rule the sexes do not differ much 
from each other in color, the males are often of a darker shade than the females. "In some species, however, the difference is conspicuous; thus the female of Sparassus smaragdulus is dullish green, whilst the adult male has the abdomen of a fine yellow with three longitudinal stripes of rich red." Darwin believes that sexual selection must take place in this group, because Canestrini has observed that the males fight for the possession of the females. He has also stated that the males pay court to the female, and that she rejects some of the males who court her, and sometimes devours them, until finally one is chosen. Darwin believed, on this evidence, that the difference in color of the sexes had been acquired by sexual selection, "though we have here not the best kind of evidence - the display by the male of his ornaments." This evidence has, however, now been supplied through the interesting observations of Mr. and Mrs. Peckham. These accurate observers have studied the courtship of the male, and observed that during the process, he twists and turns his body in such a way as to show to best advantage his colors to the female. From their account this certainly appears to be the result of his movements, but whether this is really the case, and whether the female makes any choice amongst her suitors, according to whether they are more or less brilliantly marked, we are absolutely ignorant. The following account given by Darwin should not pass unnoticed:"The male is generally much smaller than the female, sometimes to an extraordinary degree, and he is forced to be extremely cautious in making his advances, as the female often carries her coyness to a dangerous pitch. De Geer saw a male that 'in the midst of his preparatory caresses was seized by the object of his attentions, enveloped by her in a web and then devoured, a sight which, as he adds, filled him with horror and indignation.' The Rev. O. P. Cambridge accounts in the following manner for the extreme smallness of the male in the genus Nephila. 'M. Vinson 
gives a graphic account of the agile way in which the diminutive male escapes from the ferocity of the female, by gliding about and playing hide and seek over her body and along her gigantic limbs: in such a pursuit it is evident that the chances of escape would be in favor of the smallest males, while the larger ones would fall early victims; thus gradually a diminutive race of males would be selected, until at last they would dwindle to the smallest possible size compatible with the exercise of their generative functions, - in fact probably to the size we now see them, i.e. so small as to be a sort of parasite upon the female, and either beneath her notice, or too agile and too small for her to catch without great difficulty.'"

It is certainly surprising to find Darwin ascribing even this difference in size between the sexes to the action of selection. Is it not a little ludicrous to suppose that the females have reduced the males to a size too small for them to catch?

There are many cases known in the animal kingdom where there is a difference in size between the two sexes, especially in the group of insects; but I doubt very much if they are to be accounted for as the result of sexual selection. In some of these cases Darwin accounts for the larger size of the female, on account of the large number of eggs which she has to carry. In other insects where the male is larger, as in the stag-beetle, the size is ascribed to the conflicts of the males, leading to the survival of the larger individuals. In still other cases, where the males are larger, but do not fight, an explanation is admittedly wanting; but it is suggested that here there would be no necessity for the males to be smaller than the females in order to mature before them (as is supposed to happen in other species), for in these cases the individuals are not short-lived, and there would be ample time for pairing. Again, although the males of nearly all bees are smaller than the females, yet the reverse is true in 
those forms in which the females are fertilized during the marriage flight. The explanation offered is that in these forms the male carries the female, and this is assumed to require greater size on his part. This loose way of guessing, as to a possible explanation, is characteristic of the whole hypothesis of sexual selection. First one, and then another, guess is made as to the causes of the differences between the sexes. It is not shown in a single one of the instances that the postulated cause has really had anything to do with the differences in question; and the attempt to show that the theory is probable, by pointing out the large number of cases which it appears to account for, is weakened to a very great degree by the number of exceptional cases, for which an equally ready explanation of a different kind is forthcoming. This way of giving loose rein to the imagination has been the bane of the method that has followed hard on the track of Darwin's hypothesis, and for which his example has been in no small measure responsible. Thus, in the case just quoted, there are no less than four distinct conjectures made to account for the differences in size between the sexes, and each guess involves an entirely different set of processes. Considering the complicated relation of the life of organisms, it may be doubted if any of the imagined processes could bring about the result, and certainly not a single one has been shown to be a real, or a sufficient, cause in the evolutionary process. Neither the actuality of the postulated causes, nor their application to a particular case, has been shown to exist.

In the Diptera, or flies, Wallace records one interesting case of sexual difference in the genus Elaphomyia of New Guinca, in which the males are furnished with horns, which the females lack. Darwin writes:-

"The horns spring from beneath the eyes, and curiously resemble those of a stag, being either branched or palmated. In one of the species, they equal the whole body in length. 
They might be thought to be adapted for fighting, but as in one species they are of a beautiful pink color, edged with black, with a pale central stripe, and as these insects have altogether a very elegant appearance, it is perhaps more probable that they serve as ornaments."

Presumably, therefore, Darwin means these colored horns have been acquired by sexual selection.

In the Hemiptera, or bugs, both sexes of some species are "beautifully colored," and as the members of the group are often unpalatable to other animals, the color in this case is supposed to act as a warning signal.

In other cases it is stated, however, that "a small pink and green species" could hardly be distinguished from the buds on the trunks of the lime trees which this insect frequents. In this case the color appears "to be directly protective." Thus without any means of forming a correct judgment, the color of one animal is supposed to be the result of natural selection, since it resembles its surroundings, but of sexual selection if the color is present or more pronounced in one sex. Where neither view can easily be applied, the color is ascribed in a general way to the nature of the organism.

In respect to the group of Hymenoptera, or bees, Darwin records the following cases:-

"In this order slight differences in color, according to sex, are common, but conspicuous differences are rare except in the family of bees; yet both sexes of certain groups are so brilliantly colored - for instance in Chrysis, in which vermilion and metallic greens prevail - that we are tempted to attribute the result to sexual selection. In the Ichneumonidx, according to Mr. Walsh, the males are almost universally lighter-colored than the females. On the other hand, in the Tenthredinidx the males are generally darker than the females. In the Siricidre the sexes frequently differ; thus the male of Sirex juitucus is banded with orange, whilst the 
female is dark purple; but it is difficult to say which sex is the more ormamented.'

In other families of bees, differences in the color of the sexes have been recorded, and since the males have been seen fighting for the possession (?) of the females, and since bees are known to recognize differences in color, Darwin believes that :-

"In some species the more beautiful males appear to have been selected by the females; and in others the more beautiful females by the males. Consequently in certain genera, the males of the several species differ much in appearance, whilst the females are almost indistinguishable; in other genera the reverse occurs. H. Müller believes that the colors gained by one sex through sexual selection have often been transferred in a variable degree to the other sex, just as the pollen-collecting apparatus of the female has often been transferred to the male, to whom it is absolutely useless."

Although in beetles the sexes are generally colored alike, yet in some of the longicorns there are exceptions to the rule. "Most of these insects are large and splendidly colored. The males in the genus Pyrodes, which I saw in Mr. Bates's collection, are generally redder but rather duller than the females, the latter being colored of a more or less splendid golden-green. On the other hand, in one species the male is golden-green, the female being richly tinted with red and purple. In the genus Esmeralda the sexes differ so greatly in color that they have been ranked as distinct species; in one species both are of a beautiful shining green, but the male has a red thorax. On the whole, as far as I could juctge, the females of those Prionida, in which the sexes differ, are colored more richly than the males, and this does not accord with the common rule in regard to color, when acquired through sexual selection."

The great horns that rise from the heads of many male beetles are very striking cases of scxual difference, and 
Darwin compares them to the horns of stags and of the rhinoceros. They "are wonderful from their size and shapes." Darwin offers the following conjecture as to their meaning: "The extraordinary size of the horns, and their widely different structure in closely allied forms, indicate that they have been formed for some purpose; but their excessive variability in the males of the same species leads to the inference that this purpose cannot be of a definite nature. The horns do not show marks of friction, as if used for any ordinary work. Some authors suppose that as the males wander about much more than the females, they require horns as a defence against their enemies; but as the horns are often blunt, they do not seem well adapted for defence. The most obvious conjecture is that they are used by the males for fighting together; but the males have never been observed to fight; nor could Mr. Bates, after a careful examination of numerous species, find any sufficient evidence, in their mutilated or broken condition, of their having been thus used. If the males had been habitual fighters, the size of their bodies would probably have been increased through sexual selection, so as to have exceeded that of the females; but Mr. Bates, after comparing the two sexes in above a hundred species of the Copridæ, did not find any marked difference in this respect amongst well-developed individuals. In Lethrus, moreover, a beetle belonging to the same great division of the lamellicorns, the males are known to fight, but are not provided with horns, though their mandibles are much larger than those of the female."

"The conclusion that the horns have been acquired as ornaments is that which best agrees with the fact of their having been so immensely, yet not fixedly, developed, — as shown by their extreme variability in the same species, and by their extreme diversity in closely allied species. This view will at first appear extremely improbable; but we shall hereafter find with many animals standing much higher in the scale, 
namely fishes, amphibians, reptiles and bircls, that various kincls of crests, knobs, horns and combs have been developed apparently for this sole purpose."

It is asking a great deal to suppose that animals, so dull and sluggish as these beetles, are endowed with a sufficient resthetic discrimination to select in each generation those males whose horns are a little longer than the average. The resemblance of the horns to those of stags is, as Darwin points out, obvious, but in the latter case also it remains to be proven that they are the result of sexual selection, as Darwin believes to be the case; but the evidence for this belief is not much better, as we shall see in the case of the antlers of deer, than it is in these beetles.

In regard to butterflies, the males and females are both often equally brilliantly colored; in other species the differences in the sexes are very striking. Darwin states:-

"Even within the same genus we often find species presenting extraordinary clifferences between the sexes, whilst others have their sexes closely alike." The fine colors of the wings of many moths are also supposed by Darwin to have arisen through sexual selection, although the colors are usually on the lower wings, which are covered during the day by the less ornamented upper wings. It is assumed that, since the moths often begin to fly at dusk, their colors might at this time be seen and appreciated by the other sex. It should not be overlooked, however, that, in the case of some of the most highly colored moths, it is known that the males find the females through the sense of smell. Moreover, although moths are often finely colored, Darwin points out that "it is a singular fact that no British moths which are brilliantly colored, and, as far as I can discover, hardly any foreign species, differ much in color according to sex; though this is the case with many brilliant butterflies."

$Y$ et Darwin does not hesitate to conclude: "From the several foregoing facts it is impossible to admit that the brilliant 
colors of butterflies, and of some few moths, have commonly been acquired for the sake of protection. We have seen that their colors and elegant patterns are arranged and exhibited as if for display. Hence I am led to believe that the females prefer or are most excited by the more brilliant males; for on any other supposition the males would, as far as we can see, be ornamented to no purpose. We know that ants and certain lamellicorn beetles are capable of feeling an attachment for each other, and that ants recognize their fellows after an interval of several months. Hence there is no abstract improbability in the Lepidoptera, which probably stand nearly or quite as high in the scale as these insects, having sufficient mental capacity to admire bright colors. They certainly discover flowers by color."

So far as the evidence of ants having an attachment for each other is concerned, we may eliminate this part of the argument, since the evidence on which the statement is based is now regarded as only showing that ants recognize each other by their sense of smell, which resides in the antennæ. Hence the so-called fondling means only that the ants are trying by smell to determine the odor of the other individual.

Darwin points out a number of cases in which the females are more brightly colored than the males, and for such cases he reverses the process of selection, supposing that the males have been discriminating, and have not "gladly accepted any female." No explanation is offered to account for this reversal of instinct, in fact, no evidence to show that such a reversal really exists. Darwin points out that in most cases the male insect carries the female during the period of union, while in two species of butterflies, Colias cdusa and hyale, the females carry the males, and the females are here the more highly colored. He suggests that since in this case "the females take the more active part in the final marriage ceremony, so we may suppose that they likewise do so in the 
wooing; and in this case we can understand how it is that they have been rendered the more beautiful."

A most significant fact in connection with the difference in sexual coloration of butterflies did not escape Darwin's attention.

"Whilst reflecting on the beauty of many butterflies, it occurred to me that some caterpillars were splendidly colored ; and as sexual selection could not possibly have here acted, it appeared rash to attribute the beauty of the mature insect to this agency, unless the bright colors of their larve could be somehow explained. In the first place, it may be observed that the colors of caterpillars do not stand in any close correlation with those of the mature insect. Secondly, their bright colors do not serve in any ordinary manner as a protection. Mr. Bates informs me, as an instance of this, that the most conspicuous caterpillar which he ever beheld (that of a Sphinx) lived on the large green leaves of a tree on the open llanos of South America; it was about four inches in length, transversely banded with black and yellow, and with its head, legs, and tail of a bright red. Hence it caught the eye of any one who passed by, even at the distance of many yards, and no cloubt that of every passing bird."

Darwin applied to Vallace for a solution of this difficulty, and received the reply that he "thought it probable that conspicuously colored caterpillars were protected by having a nauseous taste; but as their skin is extremely tender, and as their intestines readily protrude from a wound, a slight peck from the beak of a bird would be as fatal to them as if they had been devoured. Hence, as Mr. Wallace remarks, 'distastefulness alone would be insufficient to protect a caterpillar unless some outward sign indicated to its would-be destroyer that its prey was a disgusting morsel.' Under these circumstances it would be highly advantageous to a caterpillar to be instantancously and certainly recognized as unpalatable by all birds and other animals. Thus the most gaudy colors would 
be serviceable, and might have been gained by variation and the survival of the most easily recognized inclividuals."

It need scarcely be pointed out that an occasional peck can scarcely be supposed to have led to the splendid development of color shown by some caterpillars, and Darwin confesses that at first sight this hypothesis appears bold, but nevertheless he believes that it will be found to be true. He adds, "We cannot, however, at present thus explain the elegant diversity in the colors of many caterpillars."

A most important fact in this connection should not be overlooked, namely, that in the caterpillar stage the sexual organs are so little developed that it is generally impossible at this time to distinguish between the sexes, unless a microscopic examination is made. This gives us, perhaps, a clew as to the difference between the mature sexual forms. These differences are connected with difference of sex itself. This conclusion also fits in well with the fact that during the period when the sexual organs are at the height of their development the individuals are most brilliantly colored. The primary cause of the brilliant color of many animals concerns us here only secondarily, for, since it is known that many of the lower forms are no less brilliantly and elaborately colored than are the sexes of the higher forms, it is not surprising that the sexes themselves sometimes differ in this respect.

Organs for producing sounds of different sorts are present in some insects, and these organs Darwin includes under the head of secondary sexual organs. In the group of Hemiptera, or bugs, the cicadas are the most familiar species that produce sounds. The noise is made by the males; the females are quite mute.

"With respect to the object of the music, Dr. Hartman, in speaking of the Cicada septcmdccim of the United States, says, 'the drums are now (June 6th and 7th, 185 I) heard in all directions. This I believe to be the marital summons from the males. Standing in thick chestnut sprouts about 
as high as my head, where hundreds were around me, I observed the females coming around the drumming males.' He adds, 'this season (August, I 868) a dwarf pear-tree in my garden produced about fifty larva of $C$. pruinosa; and I several times noticed the females to alight near a male while he was uttering his clanging notes.' Fritz Müller writes to me from S. Brazil that he has often listened to a musical contest between two or three males of a species with a particularly loud voice, seated at a considerable distance from each other: as soon as one had finished his song, another immediately began, and then another. As there is so much rivalry between the males, it is probable that the females not only find them by their sounds, but that, like female birds, they are excited or allured by the male with the most attractive voice."

In the flies the following cases are given by Darwin :-

"That the males of some Diptera fight together is certain; for Professor Westwood has several times seen this with the Tipula. The males of other Diptera apparently try to win the females by their music: H. Müller watched for some time two males of an Eristalis courting a female; they hovered above her, and flew from sicle to side, making a high humming noise at the same time. Gnats and mosquitoes (Culicida) also seem to attract each other by humming; and Professor Mayer has recently ascertained that the hairs on the antennx of the male vibrate in unison with the notes of a tuning-fork, within the range of the sounds emitted by the female."

In the crickets, grasshoppers, and locusts, the males "are remarkable for their musical powers"; and it is generally assumed that the souncls serve to call or to excite the female. In these forms the noise is made by rubbing the wings over each other or the legs against the wing-covers.

In some of these forms both sexes have stridulating organs, and in one case they differ to a certain extent from each 
other. "Hence we cannot suppose that they have been transferred from the male to the female, as appears to have been the case with the secondary sexual characters of many other animals. They must have been inclependently developed in the two sexes, which no doubt mutually call to each other during the season of love."

Some beetles also possess rasping organs in different parts of the body, but they cannot produce much noise by this means.

"We thus see that in the different coleopterous families the stridulating organs are wonderfully diversified in position, but not much in structure. Within the same family some species are provided with these organs, and others are desti. tute of them. This diversity is intelligible, if we suppose that originally various beetles made a shuffling or hissing noise by the rubbing together of any hard and rough parts of their bodies, which happened to be in contact; and that from the noise thus produced being in some way useful, the rough surfaces were gradually developed into regular stridulating organs. Some beetles as they move, now produce, either intentionally or unintentionally, a shuffling noise, without possessing any proper organs for the purpose."

Darwin says that he expected from analogy to find in this group also differences in the sexes, but none such were found, although in some cases the males alone possess certain characters or have them more highly developed.

It is important not to forget, when considering all questions connected with sexual selection, that in order for the result to be successful it is not only necessary that the female respond to the noises and music of the other sex, but that she choose the suitor that makes the greatest, or the most pleasing, noise. If the stridulating organs are only used by the animals in finding each other, then the case might be considered as coming under the head of natural selection. If this be granted, then it may be claimed, and apparently 
Darwin is inclined to adopt this view, that those males that make the most noise will be more likely to be heard, and possibly approached. They will, therefore, be more likely to leave descendants. We have already considered this question when dealing with the theory of natural selection in the preceding chapter and need not go over the ground again. This much may, however, be said again, that even if it is probable that these organs are of use to the animals in fincling each other, and this seems not improbable, it does not follow that the organs have been acquired through selection for this purpose.

Darwin finds his best examples of secondary sexual characters in the group of vertebrates, and since in this group the intelligence is of a higher order than in the other groups, the argument that the female chooses the more pleasing suitor is made to appear more plausible.

The elongation of the lower jaw that occurs in a few fishes at the breeding season is regarded as a secondary sexual character. On the other hand, Darwin recognizes the following difficulty in regard to the size of the males :-

"In regard to size, M. Carbonnier maintains that the female of almost all fishes is larger than the male; and Dr. Günther does not know of a single instance in which the male is actually larger than the female. With some cyprinodonts the male is not even half as large. As in many kinds of fishes the males habitually fight together, it is surprising that they have not generally become larger and stronger than the females through the effects of sexual selection. The males suffer from their small size, for, according to M. Carbonnier, they are liable to be devoured by the females of their own species when carnivorous, and no doubt by other species. Increased size must be in some manner of more importance to the females, than strength and size are to the males for fighting with other males; and this perhaps is to allow of the production of a vast number of ova." 
The last sentence implies that this particular case is to be explained by the females becoming larger on account of the number of eggs that they are to produce. But why was not the same explanation offered in the case of the spiders? It is this uncertain way of applying any explanation that suggests itself, that puts the whole method in an unfortunate light.

In many species of fish the males are brighter in color than the females. In the case of Callionymus lyra, Darwin states:-

"When fresh caught from the sea the body is yellow of various shades, striped and spotted with vivid blue on the head; the clorsal fins are pale brown with dark longitudinal bands, the ventral, caudal, and anal fins being bluish black. The female, or sordid dragonet, was considered by Linnæus, and by many subsequent naturalists, as a distinct species; it is of a dingy reddish brown, with the dorsal fin brown and the other fins white. The sexes differ also in the proportional size of the head and mouth, and in the position of the eyes; but the most striking difference is the extraordinary elongation in the male of the dorsal fin. Mr. W. Saville Kent remarks that this 'singular appendage appears from my observations of the species in confinement, to be subservient to the same end as the wattles, crests, and other abnormal adjuncts of the male in gallinaceous birds, for the purpose of fascinating their mates.' '

In the case of another fish, Cottus scorpius, there is also a great difference between the sexes, and here the males become very brilliant only at the breeding season. In other fishes, in which the sexes are colored alike, the males may become more brilliant during the breeding season. This, too, is explained by Darwin on the assumption that those males that have varied at the breeding season, so as to become more brightly colored, have been chosen in preference to the other males. 
A few cases are cited in which it has been observed that the males appear to exhibit themselves before the females, as in the following case of the Chinese Macropus:-

"The males are most beautifully colored, more so than the females. During the breeding season they contend for the possession of the females; and, in the act of courtship, expand their fins, which are spotted and ornamented with brightly colored rays, in the same manner, according to $\mathrm{M}$. Carbonnier, as the peacock. They then also bound about the females with much vivacity, and appear by 'l'étalage de leurs vives couleurs chercher à attirer l'attention des femelles, lesquelles ne paraissaient indifférentes à ce manége, elles nageaient avec une molle lenteur vers les mâles et semblaient se complaire dans leur voisinage.' ,'

In this connection Darwin makes the following general statement :-

"The males sedulously court the females, and in one case, as we have seen, take pains in clisplaying their beauty before them. Can it be believed that they would thus act to no purpose during thcir courtship? And this would be the case, unless the females exert some choice and select those males which please or excite them most. If the female exerts such choice, all the above facts on the ornamentation of the males become at once intelligible by the aid of sexual selection."

While it may readily be granted that display of the male may have for its purpose the excitement of the female, it is another question as to whether she will be more excited by the more beautiful suitor. The attentions of the male may be supposed to have a purpose, even if the female does not choose the more beautiful of her suitors. It is this last proposition, so necessary for the theory of sexual selection, that seems improbable. But even if it were probable, there are, as we shall see, other clifficulties to be overcome before we should be justified in accepting Darwin's statement quoted above, concerning the results of sexulal selection. 
In regard to those species of fish in which both sexes are equally ornamented, Darwin returns once more to his hypothesis that the color of the male, acquired through sexual selection, may be transmitted to the other sex, and then, as if in doubt on this point, he adds, that it may be the result of the "nature of the tissues and of the surrounding conditions." He even makes the suggestion, somewhat further on, that the colors may be warning, although it is confessedly unknown that these fish are cistasteful to fish-devouring animals.

In amphibians the crest on the back of the male triton, which becomes colored along its edge, is described as a secondary sexual character. The vocal sacs, present in some species of frogs, are found sometimes in both sexes, but more highly developed in the males. In other species, as in the toad, it is the male alone that sings. In the reptiles we find that the two sexes of the turtles are colored alike, and this holds also for the crocodiles. Some male turtles make sounds at the breeding season, and the same is true for the crocodiles, the males of which are said to make a "prodigous display." In snakes the males are smaller, as a rule, than the females, and the colors are more strongly pronounced, and although some snakes are very brilliantly colored, Darwin puts this down either to protective coloration, or to mimicry of other kinds of snakes. But surely the extremely brilliant colors of many snakes cannot be accounted for in any of these ways. The cause of the color of the venomous kinds, that are supposed to be imitated by the others, "remains to be explained and this may perhaps be sexual selection."

"It does not, however, follow because snakes have some reasoning power, strong passions and mutual affection, that they should likewise be endowed with sufficient taste to admire brilliant colors in their partners, so as to lead to the adornment of the species through sexual selection. Nevertheless, it is difficult to account in any other manner for the 
extreme beauty of certain species; for instance, of the coralsnakes of South America, which are of a rich red with black and yellow transverse bands."

In lizards the erectile crests of the male Anolis, the brilliant throat patches of Sitaria minor, which is colored blue, black, and red, the skinny appendages present on the throat of the little lizards of the genus Draco, which in the beauty of their colors baffle description, are given as cases of sexual adornment. In the last case cited the ornaments are present, however, in both sexes. The remarkable horns in the males of different species of chameleons are imagined to have been acquired through the battle of the males with each other.

In the group of birds we find some of the most striking cases of secondary sexual differences. The spurs, combs, wattles, horns, air-filled sacs, topknots, feathers with naked shafts, plumes, and greatly elongated feathers are all secondary sexual characters. The songs of the males, the rattling together of the quills of the peacock, the clrumming of the grouse, and the booming sounds made by the night jars while on the wing, are further examples of secondary sexual differences. The odor of the male of the Australian musk cluck is also put in the same category.

The pugnacity of many male birds is well known, and it is imagined that one of the results of the competition of the individuals of the same sex with each other has led to the development of the organs of defence and offence. The males that have been successful in these battles are then supposed to mate with the best females. In this way those secondary sexual differences, connected with the encounters of the males, are supposed to have been formed. Darwin states in this connection:-

"Even with the most pugnacious species it is probable that the pairing does not depend exclusively on the mere strength and courage of the male; for such males are generally decorated with various ornaments, which often become 
more brilliant during the breeding season, and which are sedulously displayed before the females. The males also endeavor to charm or excite their mates by love-notes, songs, and antics; and the courtship is, in many instances, a prolonged affair. Hence it is not probable that the females are indifferent to the charms of the opposite sex, or that they are invariably compelled to yield to the victorious males."

Thus a double process of selection is imagined to take place; one, the outcome of a competition of the males with each other, and the other, through a choice of the more successful males by the females, the more beautiful being supposed to be chosen.

It may be well not to lose sight of the fact that unless the selection is severe in each generation, its good effects will be lost, as has been stated in connection with the theory of natural selection. Still more important is the consideration that unless the same variations appear at the same time, in many of the surviving males, the results will be lost through crossing. These statements will show that the difficulties of the theory are by no means small, and when we are asked to believe further that another process still has been superimposed on this one, namely, the selection of the more beautiful males by the females, we can appreciate how great are the difficulties that must be overcome in order that the process may be carried out.

The love-antics and dances of male birds at the breeding season furnish many curious data. The phenomena are imagined by Darwin to be connected with sexual selection, for in the dances the males are supposed to exhibit their ornaments to the females who are imagined to choose the suitor that is most to their taste.

Hudson, who has studied the habits of birds in the field, asks some very pertinent questions in connection with their performances of different kinds. "What relation that we can see or imagine to the passion of love and the business of 


\section{96}

courtship have these dancing and vocal performances in nine cases out of ten? In such cases, for instance, as that of the scissortail tyrant-birl, and its pyrotechnic displays, when a number of couples leave their nests containing eggs and young to join in a wild aërial dance; the mad exhibitions of ypecahas and ibises and the jacana's beautiful exhibition of grouped wings; the triplet dances of the spur-winged lapwing, to perform which two birds already mated are compelled to call in a third bird to complete the set; the harmonious duets of the oven-birds and the duets and choruses of nearly all the wood-hewers, and the wing-slapping aërial displays of the whistling widgeons, - will it be seriously contended that the female of this species makes choice of the male able to administer the most vigorous and artistic slaps?"

"The believer in the theory would put all these cases lightly aside to cite the case of the male cow-bird practising antics before the female, and drawing a wide circle of melody around her, etc. . . A And this was in substance what Darwin dicl." "How unfair the argument is based on these carefully selected cases gathered from all regions of the globe and often not properly reported is seen when we turn to the book of nature and closely consider the habits and actions of all the species inhabiting any onc district." Hulson concludes that he is convinced that any one who will note the actions of animals for himself will reach the conviction, that " conscious sexual selection on the part of the female is not the cause of music and dancing performances in bircls, nor of the brighter colors and ornaments that distinguish the male."

The differences in color in the sexes of birds are classified by Darwin as follows: (I) when the males are ornamented exclusively or in a much higher degree than the females; (2) when both sexes are highly ornamented; (3) when the female is more brightly colored. A few examples of each sort may be chosen for illustration. 
"In regard to color, hardly anything need here be said, for every one knows how splendid are the tints of many birds, and how harmoniously they are combined. The colors are often metallic and iridescent. Circular spots are sometimes surrounded by one or more differently shaded zones, and are thus converted into ocelli. Nor need much be said on the wonderful difference between the sexes of many birds. The common peacock offers a striking instance. Female birds of paradise are obscurely colored and destitute of all ornaments, whilst the males are probably the most highly clecorated of all birds, and in so many different ways, that they must be seen to be appreciated. The elongated and golden-orange plumes which spring from beneath the wings of the Paradisca apoda, when vertically erected and made to vibrate, are described as forming a sort of halo, in the centre of which the head "looks like a little emerald sun, with its rays formed by the two plumes." "

Male humming-birds are almost as splendidly colored as are the birds of paradise, some having the feathers modified in a truly extraordinary way. "Almost every part of their plumage has been taken advantage of, and modified; and the modifications have been carried, as Mr. Gould showed me, to a wonderful extreme in some species belonging to nearly every subgroup. Such cases are curiously like those which we see in our fancy breeds, reared by man for the sake of ornament : certain individuals originally varied in one character, and other individuals of the same species in other characters; and these have been seized on by man and much augmented - as shown by the tail of the fantail pigeon, the hood of the jacobin, the beak and wattle of the carrier, and so forth. The sole difference between these cases is that in the one the result is due to man's selection, whilst in the other, as with humming-birds, birds of paradise, etc., it is due to the selection by the females of the more beautiful males." 
A remarkable bird of South America, the bell-bird, has a peculiar note that "can be distinguished at the distance of nearly three miles and astonishes every one who hears it.

The male is pure white, whilst the female is duskygreen; and white is a very rare color in terrestrial species of moderate size and inoffensive habits. The male, also, as described by Waterton, has a spiral tube, nearly three inches in length, which rises from the base of the beak. It is jetblack, dotted over with minute downy feathers. This tube can be inflated with air, through a communication with the palate; and when not inflated hangs down on one side. The genus consists of four species, the males of which are very distinct, whilst the females, as described by Mr. Sclater in a very interesting paper, closely resemble each other, thus offering an excellent instance of the common rule that within the same group the males differ much more from each other than do the females. In a second species ( $C$. muticollis) the male is likewise snow-white, with the exception of a large space of naked skin on the throat and round the eyes, which during the breeding season is of a fine green color. In a third species (C. tricarumculatus) the head and neck alone of the male are white, the rest of the body being chestnut-brown, and the male of this species is provided with three filamentous projections half as long as the body-one rising from the base of the beak, and the two others from the corners of the mouth."

The most familiar case of sexual difference amongst North American birds is that of the scarlet tanager, in which the male is scarlet with jet-black wings, while the female is an inconspicuous yellow-green color. Amongst domesticated animals the peafowl shows the most beautiful case of sexual differences. The magnificent tail of the male can be lifted up, so as to be seen to best advantage when the male faces the observer. Moreover the wild form, living in the forests of India, has the same gorgeous train. 
The male Argus pheasant has a remarkable series of spots, or ocelli, on the secondary wing-covers. They are concealed until the male displays them before the female. Darwin states that, while it may seem incredible that such elegant shading and exquisite patterns could have been the outcome of the taste of the female, yet the extraordinary attitude assumed by the male during courtship appears entirely pur. poseless, unless it be supposed that he is attempting to charm the female by a display of his ornamentation.

Let us pass to the second class of cases, in which both sexes are similarly and brightly colored, and in which the young have a plumage different from the adults. For example, the male and the female of the splendid scarlet ibis are alike, whilst the young are brown. The males and females of many finely colored herons are ornamented alike, and this plumage, Darwin admits, has a nuptial character. He even tries to explain this by the curious assumption, that while the color has been acquired through the selection of the males by the females, the results attained in this way have been transmitted to both sexes. We find here another example of the method so often employed by Darwin. When he meets with facts that are not in conformity with the theory, he proceeds to make a new assumption without establishing its validity. Thus, to assume that in all cases where the sexes are colored differently, the characters acquired by the males have been transmitted only to the same sex, and in those cases where the sexes are colored alike the transmission has been to both sexes, is most arbitrary.

In other cases, which are commoner than the last, the male and female have the same color, and the young in their first plumage resemble the adults. Darwin admits that here the facts are so complex that his conclusions are doubtful. The following account of the tree-sparrow shows how vague are the principles involved in the entire discussion in relation to transmission :- 
"Now with the tree-sparrow (P. montanus) both sexes and the young closely resemble the male of the house-sparrow; so that they have all been modified in the same manner, and all depart from the typical coloring of their early progenitor. This may have been effected by a male ancestor of the treesparrow having varied, firstly, when nearly mature; or secondly, whilst quite young, and by having in either case transmitted his modified plumage to the females and the young; or, thirdly, he may have varied when adult and transmitted his plumage to both adult sexes, and, owing to the failure of the law of inheritance at corresponding ages, at some subsequent period to his young."

The further admissions made in the following quotation are also significant :-

"The plumage of certain birds goes on increasing in beauty during many years after they are fully mature; this is the case with the train of the peacock, with some of the birds of paraclise, and with the crest and plumes of certain herons, for instance, the Ardea ludoricana. But it is doubtful whether the continued development of such feathers is the result of the selection of successive beneficial variations (though this is the most probable view with birds of paradise) or merely of continuous growth. Most fishes continue increasing in size, as long as they are in good health and have plenty of food; and a somewhat similar law may prevail with the plumes of birds."

We need not follow Darwin through his discussion of those cases in which the adults have a winter and a summer dress and the young resemble the one or the other in plumage, or are different from either. The discussion of these cases, confessedly very complex, adds nothing to our understanding of the theory, and little but conjecture is offered to account for the facts.

The extreme to which even conjecture can be carried may' be gathered from the following quotation, taken from the 
section dealing with cases in which the young in their first plumage differ from each other according to sex, the young males resembling more or less closely the adult males, and the young females more or less closely the adult females:

"Two humming-birds belonging to the genus Eustephanus, both beautifully colored, inhabit the small island of Juan Fernandez, and have always been ranked as specifically distinct. But it has lately been ascertained that the one which is of a rich chestnut-brown color with a golden-red head, is the male, whilst the other, which is elegantly variegated with green and white with a metallic-green head, is the female. Now the young from the first somewhat resemble the adults of the corresponding sex, the resemblance gradually becoming more and more complete.

"In considering this last case, if as before we take the plumage of the young as our guide, it would appear that both sexes have been rendered beautiful independently; and not that one sex has partially transferred its beauty to the other. The male apparently has acquired his bright colors through sexual selection in the same manner as, for instance, the peacock or pheasant in our first class of cases; and the female in the same manner as the female Rhynchæa or Turnix in our second class of cases. But there is much difficulty in understanding how this could have been effected at the same time with the two sexes of the same species. Mr. Salvin states, as we have seen in the eighth chapter, that with certain humming-birds the males greatly exceed the females in number, whilst with other species inhabiting the same country the females greatly exceed the males. If, then, we might assume that during some former lengthened period the males of the Juan Fernandez species had greatly exceeded the females in number, but that during another lengthened period the females had far exceeded the males, we could understand how the males at one time, and the females at another, might have been rendered beautiful by the selection 
of the brighter-colored individuals of either sex; both sexes transmitting their characters to their young at a rather earlier age than usual. Whether this is the true explanation I will not pretend to say; but the case is too remarkable to be passed over without notice."

The third group of cases include those in which the females are more brightly colored, or more ornamented, than the males. These cases are rare, and the differences between the sexes are never so great as when the male is the more highly colored. Wallace thinks that since in these cases the male incubates the eggs his less conspicuous colors have been acquired through natural selection. In the genus Turnix the female is larger than the male, and lacks the black on the throat and neck, and the plumage as a whole is lighter than that of the male. The natives assert that the females after laying their eggs associate in flocks, and leave the males to do the incubating; and from other evidence Darwin thinks that this is true.

In three species of painted snipe the females "are not only larger but much more richly colored than the males," and the trachea is more convoluted in some species. "There is also reason to believe that the male undertakes the duty of incubation." In the dotterel plover the female is larger and somewhat more strongly colored. The males take at least a share in the incubation. In the common cassowary the female is larger and the skin of the head more brightly colored than in the male. The female is pugnacious during the breeding season and the male sits on the eggs. The female emu is large and has a crest. She is more courageous and pugilistic and makes a deep, hollow, guttural boom. The male is more docile and can only hiss or croak. He not only incubates the eggs, but defends the young against their own mother. "So that with this emu we have a complete reversal not only of the parental and incubating instincts, but of the usual moral qualities of the two sexes; the females 
being savage, quarrelsome, and noisy, the males gentle and good. The case is very different with the African ostrich, for the male is somewhat larger than the female and has finer plumes with more strongly contrasted colors; nevertheless he undertakes the whole duty of incubation."

Darwin attempts to explain these reversals of instincts on the assumption that the males have turned the tables on the females, and have themselves done the selecting; and incidentally, it may be pointed out in passing, they have had to pay the penalty by incubating the eggs.

In the group of mammals, Darwin thinks that the male wins the female by conquering other males rather than by charming her through his display. The males, even when unarmed, engage in desperate conflicts with each other, and sometimes kill, but more often only wound, their fellows. The secondary sexual characters of the males have been acquired, therefore, by natural selection applied to one sex, and less frequently through the choice of the female. Since we are here more especially concerned with the latter class of phenomena, we may examine only a few cases under the first head.

The horns of stags are used by them in their conflicts with each other; the tusks of the elephant make this animal the most dangerous in the world, when in must. The horns of bulls, the canine teeth of many mammals, the tusks of the walrus, are further examples of organs which have been, according to Darwin, acquired through the competitions of the males with each other.

The voices of mammals are used for various purposes, "as a signal of danger, as a call from one member of the troup to another, and from the mother to her lost offspring, or from the latter for protection."

"Almost all male animals use their voices much more during the rutting season than at any other time; and some, as the giraffe and porcupine, are said to be completely mute 
excepting at this season. As the throats (i.c. the larynx and thyroid bodies) of stags periodically become enlarged at the beginning of the breeding season, it might be thought that their powerful voices must be somehow of high importance to them; but this is very doubtful. From information given to me by two experienced observers, Mr. McNeill and Sir P. Egerton, it seems that young stags under three years ofd do not roar or bellow; and that the old ones begin bellowing at the commencement of the breeding season, at first only occasionally and moderately, whilst they restlessly wander about in search of the females. Their battles are prefaced by loud and prolonged bellowing, but during the actual conflict they are silent. Animals of all kinds which habitually use their voices utter various noises under any strong emotion, as when enraged and preparing to fight; but this may merely be the result of nervous excitement, which leads to the spasmodic contraction of almost all the muscles of the body, as when a man grinds his teeth and clenches his fists in rage or agony. No doubt stags challenge each other to mortal combat by bellowing; but those with the more powerful voices, unless at the same time the stronger, better-armed, and more courageous, would not gain any advantage over their rivals."

"Some writers suggest that the bellowing serves as a call to the female; but the experienced observers above quoted inform me that female deer do not search for the male, though the males search eagerly for the females, as indeed might be expected from what we know of the habits of other male quadrupeds. The voice of the female, on the other hand, quickly brings to her one or more stags, as is well known to the hunters who in wild countries imitate her cry.

"As the case stands, the loud voice of the stag luring the breeding season does not seem to be of any special service to him, either cluring his courtship or battles, or in any other way. But may we not believe that the frequent use of the voice, under the strong excitement of love, jealousy, and rage, 
continued during many generations, may at last have produced an inherited effect on the vocal organs of the stag, as well as of other male animals? This appears to me, in our present state of knowledge, the most probable view."

Here once more we find that Darwin makes use, as a sort of last resort, of the principle of the inheritance of acquired characters. As long as the theory of selection, in any of its forms, appears to offer a satisfactory solution, we find the facts used in support of this theory, but as soon as a difficulty arises the Lamarckian theory is brought to the front. It is this shifting, as we have already more than once pointed out, that shows how little real basis there is for the theory of sexual selection.

The male gorilla has a tremendous voice, and he has, as has also the orang, a laryngeal sac. One species of gibbon has the power of producing a correct octave of musical notes.

"The vocal organs of the American Mycetes caraya are one-third larger in the male than in the female, and are wonderfully powerful. These monkeys in warm weather make the forests resound at morning and evening with their overwhelming voices. The males begin the dreadful concert, and often continue it during many hours, the females sometimes joining in with their less powerful voices. An excellent observer, Rengger, could not perceive that they were excited to begin by any special cause; he thinks that, like many birds, they delight in their own music, and try to excel each other. Whether most of the foregoing monkeys have acquired their powerful voices in order to beat their rivals and charm the females - or whether the vocal organs have been strengthened and enlarged through the inherited effects of long. continued use without any particular good being thus gained - I will not pretend to say; but the former view, at least in the case of the Hylobates agilis, seems the most probable."

The odor of some mammals is confined to, or more developed, in the males; but in some forms, as in the skunk, it is 
present in both sexes. In the shrew mice, abdominal scent glands are present, but since these mice are rejected by birds of prey, their glands probably serve to protect them; "nevertheless the glands become enlarged in the males during the breeding season." In many other quadrupeds the scent glands are of the same size in both sexes, and their function is unknown.

"In other species the glands are confined to the males, or are more developed than in the females; and they almost always become more active during the rutting season. At this period the glands on the sides of the face of the male elephant enlarge, and emit a secretion having a strong musky odor. The males, and rarely the females, of many kinds of bats have glands and protrudable sacs situated in various parts; and it is believed that these are odoriferous.

"The rank effluvium of the male goat is well known, and that of certain male deer is wonderfully strong and persistent. Besides the general odor, permeating the whole body of certain ruminants (for instance, Bos moschatus) in the breeding season, many deer, antelopes, sheep, and goats, possess odoriferous glands in various situations, more especially on their faces. The so-called tear-sacs, or suborbital pits, come under this head. These glands secrete a semifluid fetid matter which is sometimes so copious as to stain the whole face, as I have myself seen in an antelope. They are 'usually larger in the male than in the female, and their development is checked by castration.' According to Desmarest they are altogether absent in the female of Antilope subsutturosa. Hence, there can be no doubt that they stand in close relation with the reproductive functions. They are also sometimes present, and sometimes absent, in nearly allied forms. In the adult male musk-deer (Moschus moschifons), a naked space round the tail is bedewed with an odoriferous fluid, whilst in the adult female and in the male until two years old, this space is covered with hair, and 
is not odoriferous." Darwin believes in these cases that the odor serves to attract the females. He admits that here, "active and long-continued use cannot have come into play as in the case of the vocal organs." He concludes, therefore, that "the odor emitted must be of considerable importance to the male, inasmuch as large and complex glands, furnished with muscles for everting the sac, and for closing or opening the orifice, have in some cases been developed. The development of these organs is intelligible through sexual selection, if the most odoriferous males are the most successful in winning the females, and in leaving offspring to inherit their gradually perfected glands and colors."

There is sometimes a difference in the mammals in the hair of the two sexes both in amount and in color. In some species of goats the males have a beard, in others it is present in both sexes. The bull, but not the cow, has curly hair on the forehead. In some monkeys the beard is confined to the male, as in the orang; in other species it is only larger in the males.

"The males of various members of the ox family (Bovidæ), and of certain antelopes, are furnished with a dewlap, or great fold of skin on the neck, which is much less developed in the female.

"Now, what must we conclude with respect to such sexual differences as these? No one will pretend that the beards of certain male goats, or the dewlap of the bull, or the crests of hair along the backs of certain male antelopes, are of any use to them in their ordinary habits.

"Must we attribute all these appenclages of hair or skin to mere purposeless variability in the male? It cannot be denied that this is possible; for in many domesticated quadrupeds, certain characters, apparently not derived through reversion from any wild parent form, are confined to the males, or are more developed in them than in the females 
- for instance, the hump on the male zebu cattle of India, the tail of fat-tailed rams, the arched outline of the forehead in the males of several breeds of sheep, and, lastly, the mane, the long hairs on the hind-legs, and the dewlap of the male of the Berbura goat."

In these cases and in others that Darwin cites, which seem clearly to inclicate that some of these secondary sexual characters are not the result of sexual selection, he concludes, "that they must be due to simple variability, together with sexually limited inheritance.

"Hence it appears reasonable to extend this same view to all analogous cases with animals in a state of nature. Nevertheless I cannot persuade myself that it generally holds good, as in the case of the extraordinary development of hair on the throat and fore-legs of the male Ammotragus, or in that of the immense beard of the male Pithecia. Such study as I have been able to give to nature makes me believe that parts or organs which are highly developed, were acquired at some period for a special purpose. With those antelopes in which the adult male is more strongly colored than the female, and with those monkeys in which the hair on the face is elegantly arranged and colored in a diversified manner, it seems probable that the crests and tufts of hair were gained as ornaments; and this I know is the opinion of some naturalists. If this be correct, there can be little doubt that they were gained, or at least modified through sexmal selection; but how far the same view may be extended to other mammals is cloubtful."

The astonishing colors in some of the monkeys cannot be passed over without comment.

"In the beautiful Coropithens dirna, the head of the aclult mate is of an intense black, whilst that of the female is dark gray; in the former the fur between the thighs is of an elegant fawn-color, in the latter it is paler.

"In the Corcopithicus cynosums and griscoviridis one part 
of the body, which is confined to the male sex, is of the most brilliant blue or green, and contrasts strikingly with the naked skin on the hincler part of the body, which is vivid red.

"Lastly, in the baboon family, the adult male of $C_{y}$ nocophalus hamadryas differs from the female not only by his immense mane, but slightly in the color of the hair and of the naked callosities. In the drill(C.lcucophaus) the females and young are much paler-colored, with less green, than the adult males. No other member in the whole class of mammals is colored in so extraordinary a manner as the adult male mandrill ( $C$. mormon). The face at this age becomes of a fine blue, with the riclge and tip of the nose of the most brilliant red. According to some authors, the face is also marked with whitish stripes, and is shaded in parts with black, but the colors appear to be variable. On the forehead there is a crest of hair, and on the chin a yellow beard. 'Toutes les parties supérieures de leurs cuisses et le grand espace nu de leurs fesses sont également colorés du rouge le plus vif, avec un mélange de bleu qui ne manque réellement pas d'élégance.' When the animal is excited all the naked parts become much more vividly tinted."

Darwin sums up the evidence in regard to the differences in color between the male and female in the following statement :-

"I have now given all the cases known to me of a difference in color between the sexes of mammals. Some of these may be the result of variations confined to one sex and transmitted to the same sex, without any good being gained, and therefore without the aid of selection. WVe have instances of this with our domesticated animals, as in the males of certain cats being rusty-red, whilst the females are tortoise-shell colored. Analogous cases occur in nature: Mr. Bartlett has seen many black varieties of the jaguar, leopard, vulpine phalanger, and wombat; and he is certain that all or nearly all these animals, were males. On the 
other hand, with wolves, foxes, and apparently American squirrels, both sexes are occasionally born black. Hence it is quite possible that with some mammals a difference in color between the sexes, especially when this is congenital, may simply be the result, without the aid of selection, of the occurrence of one or more variations, which from the first were sexually limited in their transmission. Nevertheless it is improbable that the diversified, vivid, and contrasted colors of certain quadrupeds, for instance, of the above monkeys and antelopes, can thus be accounted for."

Finally, the case of man must be considered from the point of view of sexual selection, for Darwin claims that man has acquired a number of his secondary sexual characters in this way. For instance, the beard is an excellent case of a secondary sexual character. Darwin's interpretation is that the beard has been retained, or even developed, through the selection by the females of those males that had this outgrowth best developed. Conversely, the absence of hair on the face of the female is supposed by Darwin to have been brought about by men selecting those women having less hair on their faces. The greater intellect, energy, courage, pugnacity, and size of man are the outcome of the competition of the males with each other, since the individual excelling in these qualities will be able to select the most desirable wife, or wives, and it is assumed will, therefore, leave more descendants. The standard of beauty has been kept up by men selecting the most beautiful women in each generation (the fate of the other married women is ignored), and this beauty is supposed to have been transmitted primarily to their daughters, but also to their sons.

Although all these forms of selection are imagined to be acting in man, either alternately or simultaneously, yet Darwin recognizes in man a number of checks to the action of sexual selection: amongst savages, the so-called communal marriages; second, infanticide, generally of the young 
females, which appears in some races to be practised to an astonishing degree ; third, early betrothals; fourth, the holding of women as slaves.

When we recall that selection to be effective can only be carried out under very exacting conditions, we cannot but be appalled at the demands made here on our credulity. The choice of the women has produced the beard of man, the choice of man the absence of a beard in women; the competition of the males with each other is leading at the same time to the development of at least half a dozen qualities that are supposed to be male specialities, and while all this is going on the results are being checked sometimes by one means, sometimes by another. Moreover, even this is not all that we are asked to accept, for there are several other qualities of the male that are put down as secondary sexual characters. For example, let us examine what Darwin has to say in regard to the development of the voice, and of singing in man.

In man the vocal cords are about a third longer than in woman and his voice deeper. Emasculation arrests the development of the vocal apparatus, and the voice remains like that of a woman. This difference between the sexes, Darwin thinks, is due probably to long-continued use by the male "under the excitement of love, rage, and jealousy." In other words, an appeal is again made to the Lamarckian theory, and in this case to explain the origin of an organ that conforms to all the requirements of the secondary sexual characters.

"The capacity and love for singing, or music, though not a sexual character in man," in the sense of being confined to one sex, yet is supposed to have arisen through sexual selection in the following way: "Human song is generally admitted to be the basis or origin of instrumental music. As neither the enjoyment nor the capacity of producing musical notes are faculties of the least use to man in refer- 
ence to his daily habits of life, they must be ranked amongst the most mysterious with which he is endowed."

Man is supposed to have possessed this faculty of song from a very remote time, and even the most savage races make musical sounds, although we do not enjoy their music, or they ours.

"We see that the musical faculties, which are not wholly deficient in any race, are capable of prompt and high development, for Hottentots and Negroes have become excellent musicians, although in their native countries they rarely practise anything that we should consider music. Hence the capacity for high musical development, which the savage races of man possess, may be due either to the practice by our semi-human progenitors of some rude form of music, or simply to their having acquired the proper vocal organs for a different purpose. But in this latter case we must assume, as in the above instance of parrots, and as seems to occur with many animals, that they already possessed some sense of melocly."

Darwin sums up the evidence in the two following statements, the insufficiency of which to explain the phenomena is I think only too obvious: "All these facts in respect to music and impassioned speech become intelligible to a certain extent, if we assume that musical tones and rhythm were used by our half-human ancestors, during the season of courtship, when animals of all kinds are excited not only by love, but by the strong passions of jealousy, rivalry, and triumph. From the deeply laid principle of inherited associations, musical tones in this case would be likely to call up vaguely and indefinitely the strong emotions of a long past age." Thus the difficulty is shifted to the shoulders of our long lost savage ancestors; or even, in fact, to our simian forefathers, as the following paragraph indicates:-

"As the males of several quadrumanous animals have their vocal organs much more developed than in the females, 


\section{Darwin's Theory of Sexual Selection}

and as a gibbon, one of the anthropomorphous apes, pours forth a whole octave of musical notes and may be said to sing, it appears probable that the progenitors of man, either the males or females or both sexes, before acquiring the power of expressing their mutual love in articulate language, endeavored to charm each other with musical notes and rhythm. So little is known about the use of the voice by the Quadrumana during the season of love, that we have no means of judging whether the habit of singing was first acquired by our male or female ancestors. Women are generally thought to possess sweeter voices than men, and as far as this serves as any guide, we may infer that they first acquired musical powers in order to attract the other sex. But if so, this must have occurred long ago, before our ancestors had become sufficiently human to treat and value their women merely as useful slaves. The impassioned orator, bard, or musician, when with his varied tones and cadences he excites the strongest emotions in his hearers, little suspects that he uses the same means by which his halfhuman ancestors long ago aroused each other's ardent passions during their courtship and rivalry."

We have now examined in some detail the evidence that Darwin has brought forward in support of his hypothesis of sexual selection. A running comment has been made while considering the individual cases, but it may be well to sum up the matter by briefly indicating the reasons why the hypothesis seems incompetent to explain the facts.

General Criticism of the Theory of Sexual Selection

I. Some of the objections that apply to the theory of natural selection apply also with equal force to the theory of sexual selection in so far as the results in both cases are supposed to be the outcome of the selection of individual, or fluctuating, variations. If these variations appear in only 
a few individuals, their perpetuation is not possible, since they will soon disappear through crossing. It would be, of course, preposterous to suppose that at any one time only those few individuals pair and leave descendants that have the secondary sexual characters developed to the highest point, but if something of this sort does not occur, the extreme of fluctuating variations cannot be maintained. Even if half of the individuals are selected in each generation, the accumulation of a variation in a given direction could not go very far. The assumption, however, that only half of all the individuals that reach maturity breed, and that all of these are chosen on account of the special development of their secondary sexual characters, seems preposterous. Furthermore, if it is assumed that the high development of the new character appears in a large number of individuals, then it is not improbable that its continued appearance might be accounted for without bringing in, at all, the hypothesis of sexual selection.

2. But even supposing that the females select the most beautiful males, then, since in the rast majority of higher animals the males and the females are in equal numbers, the others will also be able to unite with each other in pairs after this first selection has taken place. Nothing will therefore be gained in the next generation. It is interesting to see how Darwin attempts to meet this argument. He tries to show in the case of birds, that there are always unpaired individuals, but since the few facts that he has been able to collect show that there are as many additional females as males, the argument proves too much. A few species are polygamous, one male having a number of female birds; but on this basis we can only account, at best, for the development through competition of the organs of offence and defence used to keep away the weaker males. Yet it is just amongst these birds that we often find the ornamental characters well developed. In fact, since all the females in such cases are selected, and 
since they will transmit the characters of all the males, it is evident that the secondary sexual characters could not be formed in the way imagined.

3. If the female fails to select only the more ornamental males, no result will follow. It has not been shown that she is capable of making such a choice, and in the lower forms particularly, it does not seem probable that this is done. The argument that Darwin often employs, namely, that unless she does select, the display of the males before her is meaningless, is not to the point. So far as we can detect the "cause" of the display of the male, it appears to be due to his own excitement; and even if we go so far as to admit that the "purpose" is to attract the other sex, it still does not in the least follow that the most ornamental male is selected, and unless this occurs the display has no bearing on the hypothesis of sexual selection.

4. The two forms of sexual selection, namely, competition of the males with one another (really one form of natural selection), and the selection of the most ornamental or gifted individuals, are both used by Darwin to explain secondary sexual characters, the one for organs of offence and defence, and the other for ornamental characters. If we fully appreciate the difficulties that any theory of selection meets with, we shall realize how extraordinarily complex the action must be, when two such processes are carried out at the same time, or even during alternating periods.

5. It has been objected to Darwin's theory of sexual selection, that he suddenly reverses its mode of action to explain those cases in which the female is the stronger and more ornamented sex; but if, as Darwin shows, the instincts of the male have also changed, and have become more like those of the female, I can see no inherent difficulty in this way of applying the theory. A much more serious objection, it seems to me, is that the male is supposed to select the female for one set of characteristics, and the female to select the 
male for another set. It sounds a little strange to suppose that women have caused the beard of man to develop by selecting the best-bearded individuals, and the compliment has been returned by the males selecting the females that have the least amount of beard. It is also assumed that the results of the selection are transmitted to one sex only. Unless, in fact, the character in question were from the beginning peculiar to only one sex as to its inheritance, the two sexes might go on forever selecting at cross-purposes, and the result would be nothing.

6. The development, or the presence, of the resthetic feeling in the selecting sex is not accounted for on the theory. There is just as much need to explain why the females are gifted with an appreciation of the beautiful, as that the beautiful colors develop in the males. Shall we assume that still another process of selection is going on, as a result of which those females are selected by the males that appreciate their unusual beauty, or that those females whose taste has soared a little higher than that of the average (a variation of this sort having appeared) select males to correspond, and thus the two continue heaping up the ornaments on one side and the appreciation of these ormaments on the other? No doubt an interesting fiction could be built up along these lines, but would any one believe it, and, if he did, could he prove it ?

Darwin assumes that the appreciation on the part of the female is always present, and he thus simplifies, in appearance, the problem, but he leaves half of it unexplained.

7. It has been pointed out, that it is important to distinguish between the possible excitement of the female by the display or antics of the male, and the selection of the more beautiful or agile performer. Darwin himself records a few cases, which plainly show that the more beautiful is not always the more successful. It has also been suggested that the battles of the males are sometimes sham performances, and even when they are real, if the less vigrorous do not remain 
to be clestroyed but run away, they live to find mates of their own. In fact, the conduct of the males at the breeding season appears to be much more the outcome of their own excitement than an attempt to attract the females.

8. There is another side to the question, the importance of which is so great, that it is surprising that Darwin has not taken any notice of it. If, in order to bring about, or even maintain, the results of sexual selection, such a tremendous elimination of inclividuals must take place, it is surprising that natural selection would not counteract this by destroying those species in which a process, so useless for the welfare of the species, is going on. It is curious that this has not been realized by those who believe in both of these two hypotheses.

9. What has just been said applies also with almost equal force to the development of such structures as the horns of deer, bison, antelopes, and the brilliant colors of many insects and bircls. If in nature, competition between species takes place on the scale that the Darwinian theory of natural selection postulates, such forms, if they are much exposed, would be needlessly reduced in numbers in the process of acquiring these structures. So many individuals would have been at such a clisadvantage in breeding, that if competition is as severe as the theory of natural selection postulates, these species could hardly be expected to compete successfully with other species in which sexual selection was not taking place.

Io. Darwin admits that, in certain cases, external conditions may have acted directly to produce the colors in certain forms, and if these were not injurious he thinks they might have become constant. Such cases are left unexplained in the sense that they are not supposed to be adaptations to anything in particular. That colors produced in this way might afterward be found useful, irrespective of how they arose, is admitted as one of the ways in which sexual differences may have arisen. 
I I. It is baffling to find Darwin resorting to the Lamarckian explanation in those cases in which the improbability of the hypothesis of sexual selection is manifest. If either principle is true, we should expect it to apply to all phenomena of the same sort; yet Darwin makes use of the Lamarckian principle, in the hypothesis of sexual selection, only when difficulties arise.

I2. In attempting to explain the development of the musical sense in man, it is elear that the hypothesis of sexual selection fails to give a satisfactory explanation. To suppose that the genius of a Beethoven or of a Mozart could have been the result of a process of sexual selection is too absurd to discuss. Neither the power of appreciation nor of expression in music could possibly have been the outcome of such a process, and it cloes not materially help the problem to refer it back to a troop of monkeys making the woods hideous with their cries.

We come now to some of the special cases to which Darwin's hypothesis has been applied.

I3. In one case at least, it is stated that a bird living on the ground might have acquired the color of the upper surface of the body through natural selection, while the under surface of the males of the same species might have become ornamented through the action of sexual selection. Thus in one and the same individual the two processes are supposed to have been at work, and it does not lessen the difficulty very much by supposing the two processes to have been carried out at different times, because it is evident that what had been gained at one time by one process might become lost while the color of certain parts was being acquired through the other process.

14. Darwin points out that "the plumage of certain birds goes on increasing in beaty during many years after they are fully mature," as in the peacock, and in some of the birds of paradise, and with the plumes and crests of some herons. 
This is explained as possibly merely the result of "continued growth." The improbability of selection is manifest in these cases, but if "continued growth" can accomplish this much, why may not the whole process be also the outcome of such growth? At any rate, whatever the explanation is, it is important to find a case of a secondary sexual character that the hypothesis obviously is insufficient to explain.

15. It is admitted in a number of cases, as in the stag for instance, that, although the larynx of the male is enlarged, this is not, in all probability, the outcome of sexual selection, but in other forms this same enlargement is ascribed to the selection process.

I6. It is admitted that in none of the highly colored British moths is there much difference according to sex, although when a difference of color is found in butterflies this is put down to the action of sexual selection. If such wonderful colors as those of moths can arise without the action of selection, why make a special explanation for those cases in which this difference is associated with sex?

17. It is well known that birds sing at other times of the year than at the breeding season, and an attempt is made to account for this in that birds take pleasure in practising those instincts that they make use of at other times, as the cat plays with the captive mouse. Does not this suggest that, if they had certain instincts, they would be more likely to employ them at the times when their vitality or excitement is at its highest without regard to the way in which they have come by them?

I8. The color of the iris of the eyes of many species of hornbills is said to be an intense crimson in the males, and white in the females. In the male condor the eye is yellowish brown, and in the female a bright red. Darwin admits that it is doubtful if this difference is the result of sexual selection, since in the latter case the lining of the mouth is black in the males, and flesh-colored in the females, which does not 
affect the external beauty. Yet if these colors were more extensive and on the exterior, there can be little doubt that they would have been explained as due to sexual selection.

19. When the females in certain species of birds differ more from each other than they do from their respective males, the case is compared to "those inexplicable ones, which occur independently of man's selection in certain subbreeds of the game-fowl, in which the females are very different, whilst the males can hardly be distinguished." Here then is a case of difference in color associated with sex, but not the outcome of sexual selection.

20. The long hairs on the throat of the stag are said possibly to be of use to him when hunted, since the dogs generally seize him by the throat, "but it is not probable that the hairs were specially developed for this purpose; otherwise the young and the females would have been equally protected." Here also is a sexual difference that can scarcely be ascribed to selection.

Some cases of differences in color between the sexes " may be the result of variations confined to one sex, and transmitted to the same sex without any good being gained, and, therefore, without the aid of selection. We have instances of this with our domesticated animals, as in the males of certain cats being rusty-red while the females are tortoise-shell colored. Analogous cases occur in nature: Mr. Bartlett has seen many black varieties of the jaguar, leopard, vulpine phalanger, and wombat; and he is certain that all or nearly all of these animals were males." If changes of this sort occur, associated with one sex, why is there any need of a special explanation in other cases of difference?

In the light of the many difficulties that the theory of sexual selection meets with, I think we shall be justified in rejecting it as an explanation of the secondary sexual differ- 
ences amongst animals. Other attempts to explain these differences have been equally unsuccessful. Thus Wallace accounts for them as due to the excessive vigor of the male, but Darwin's reply to Wallace appears to show that this is not the cause of the difference. He points out that, while the hypothesis might appear plausible in the case of color, it is not so evident in the case of other secondary sexual characters, such, for instance, as the musical apparatus of the males of certain insects, and the difference in the size of the larynx of certain birds and mammals.

Darwin's theory served to draw attention to a large number of most interesting differences between the sexes, and, even if it prove to be a fiction, it has done much good in bringing before us an array of important facts in regard to differences in secondary sexual characters. More than this I do not believe it has done. The theory meets with fatal objections at every turn.

In a later chapter the question will be more fully discussed as to the sense in which these secondary sexual differences may be looked upon as adaptations. 


\section{CHAPTER VII}

THE INHERITANCE OF ACQUIRED CHARACTERS AS

A FACTOR IN EVOLUTION

LAMARCK's THEORY

ONE of the most striking and peculiar characteristics of living things is that through use a part is able to carry out a particular function better than before, and in some cases the use of the part leads to its increase in size. Conversely, disuse leads to the decrease of a part in size. We are perfectly familiar with this process in ourselves as applied to our nervous system and muscles.

It is not surprising that the idea should have arisen that, if the results of the use of a part are inherited by the next generation, the adaptation of organisms might be explained in this way. The presence of the organs of touch, in those parts of the body that are more likely to come into contact with foreign bodies, offers a striking parallel to the perfecting of the sensation of touch that can be brought about through the use of any part. The development of eyes only on the exposed parts of the body, as on the tentacles of the sedentary annelicts, or along the margin of the mantle of a bivalve mollusk, suggests that there may be some direct connection between their presence in these regions and the effect of light on the parts. In fact, ever since the time of Lamarck, there have been many zoologists who have claimed that many of the adaptations of organisms have arisen in this way, that is, through the inheritance of the characters acquired through use. In general this theory is summed up in the phrase, "the inheritance of acquired characters." 
This view is prominently associated with the name of Lamarck, who held, however, a different view in regard to the origin of some of the other structures of the organism. Moreover, Erasmus Darwin, even before Lamarck, had suggested the principle of the inheritance of acquired characters.

As has just been said, Lamarck held that the inheritance of acquired characters was only one of the ways in which animals have become changed, and he clearly stated that in the case of all plants and of some of the lower animals the change (evolution) which he supposed them to undergo was due to the general influence of the environment. Since plants and the lower animals (as he supposed) have no central nervous system, or at least no such well-defined nervous system as have the higher animals, Lamarck thought that they could not have evolved in the same way as have the higher animals. We now know that, so far as the lower animals, at least, are concerned, there was no need for such a distinction, since many of their responses are like those of the higher animals. This distinction that Lamarck made is responsible, no doubt, for a misconception that was long held in regard to a part of his views. It is often stated that he supposed the desire of the animal for a particular part has led to the development of that part; while in reality he only maintained the desire to use a particular organ to fulfil some want led to its better development through exercise, and the result was inherited. Lamarck also supposed that the decrease in use of a part which leads to its decrease in size accounts for the degeneration of organs.

Lamarck first advanced his theory in I80I, when he cited the following examples in its favor. A bird, driven through want to the water to find its food, will separate its toes when they strike the water. The skin uniting the bases of the toes will be stretched in consequence, and in this way the broad membrane between the toes of ducks and geese has been acquired. The toes of a bird that is in the habit of perching 
on a tree become elongated in consequence of becoming stretched, hence has arisen the foot with the long toes characteristic of arboreal birds.

Shore-birds, "which do not care to swim," but must approach the water in order to obtain food, will be in danger of sinking into the mud, "but, wishing to act so that their body shall not fall into the liquid, they will contract the habit of extending and lengthening their legs." Hence have arisen the stiltlike legs of shore-bircls.

These ideas were more fully elaborated in the following year. He added the further examples: Our dray-horses have arisen through the use to which they have been put, and the race-horse also, which has been used in a different way. Cultivated plants, on the contrary, are the result of the new environment to which they have been subjected.

In the "Philosophie Zoologique," published in I 809, Lamarck has much more fully developed his theory. Here he combats strenuously the idea that species are fixed. His point of view may be judged by the following propositions, which he believes can be established:-

I. That all organized bodies of our globe are veritable productions of nature, which she has successively produced in the course of a long time.

2. That in her progress nature began, and begins still every day, to produce the simplest organisms, and that she still produces directly the same primitive kinds of organizations. This process has been called spontaneous generation.

3. That the first beginning of animals and of plants takes place in favorable localities and under favorable circumstances. An organic movement having once established their production, they have of necessity gradually developed their organs, and have become diversified in the course of time.

4. That the power of growth of each part of the body being inherited as a consequence of the first effect of life, 
different modes of multiplication and of regeneration have arisen, and these have been conserved.

5. That with the aid of sufficient time and of favorable circumstances the changes that have taken place on the surface of the globe have called forth new structures and new habits, and in consequence have modified the organs of the body, and made animals and plants such as we see them at the present day.

6. Finally, as a result of these changes that living bodies have been forced to undergo, species have been formed, but these species have only a relative constancy, and are not as ancient as is nature herself. If the environment remains the same, species also remain the same, as is exemplified by the animals living at present in Egypt, which are exactly like those living there in ancient times.

Lamarck concludes that the appearance of stability is always mistaken by the layman for the reality, because, in general, every one judges things relatively to himself. In fact, species are not absolutely constant, but are so only temporarily. "The influence of the environment is continuous and always active, but its effects may only be recognized after a long time." The irregularity and the complexity of the organization of animals is the outcome of the infinitely diversified circumstances to which they have been subjected. These changes, Lamarck claims, do not directly cause modifications in the form of animals, ${ }^{1}$ but bring about changes in their needs, and changes in their needs bring about changes in their actions. If the needs remain the same, the acquired actions become habits. These habitual actions lead to the use of certain parts in preference to others, and this in turn to an alteration in form and structure. The individuals so changed breed together and leave descendants that inherit the acquired modification.

Curiously enough, Lamarck follows up this argument by

1 This is clearly meant to be applied only in the case of higher animals. 
citing some cases amongst plants that have been changed directly by the action of the environment. He says that since plants have no motions they have consequently no habits, but they are developed by changes in their nutrition, etc., and this brings about the superiority of some of the vital movements over others.

Amongst domestic animals Lamarck cites the case of the dog, that has come from a wild form like the wolf, but having been carried into different countries has acquired different and new habits, and this has led to the formation of new races, such as the bulldog, greyhound, pug-dog, spaniel, etc.

Lamarck's argument shifts so often back and forth from animals to plants, that it is clear that in his own mind he did not see any important difference between the action of the environment on plants, and the use of the organs of the animal. He gives in this same connection his oft-quoted summary of what he calls the two laws of nature "which observation always establishes."

First Law. In every animal, that has not passed beyond the term of its development, the frequent and sustained use of any organ strengthens it, develops it, increases its size, and gives it strength proportionate to the length of time of its employment. On the other hand, the continued lack of use of the same organ sensibly weakens it; it deteriorates, and its faculties diminish progressively until at last it disappears.

Sccond Law. Nature preserves everything that she has caused the individual to acquire or to lose by the influence of the circumstances to which the race has been for a long time exposed, and consequently by the influence of the predominant use of certain organs (or in consequence of its continued disuse). She does this by the generation of new individuals which are produced with the newly acquired organs. This occurs, provided that the acquired changes were common to the two sexes, or to the individuals that produced the new forms. 
These laws are, Lamarck says, fundamental truths which cannot be misunderstood except by those who have never observed or followed nature in her operations. He insists that it is a mistake to suppose that the parts are responsible for the functions, for it is easy to demonstrate that it is the needs and uses of the organs that have caused the parts to develop.

If it is supposed, he continues, that these laws are hypothetical, they may be demonstrated by the following facts: The adult baleen whale is without teeth, although in the foetus teeth are present, concealed in the jaws. The loss of the teeth is the result of the whale swallowing its food without first masticating it. The ant-eater is also without teeth, and has also the habit of swallowing its food without chewing it. The mole has very small eyes, and this is the result of its having made very little use of them, since its habits are subterranean. Another animal, the aspalax, has only the rudiments of eyes, and has almost completely lost the power of sight. This animal also lives underground like the mole.

Proteus, an aquatic salamander living in deep caves, has only rudimentary eyes. In these latter cases it is the disuse of the eye that has led to its degeneration. This is proven, Lamarck adds, by the fact that the organs of hearing are never in this condition, because sound vibrations penetrate everywhere, even into the densest bodies.

It is a part of the plan of organization of the reptiles that they have four legs; but the snakes, although belonging to this group, have no legs. This absence of legs is explained by their having acquired the habit of gliding over the ground, and of concealing themselves in the grass. Owing to their repeated effort to elongate themselves, in order to pass through narrow spaces, their bodies have become drawn out. Under these circumstances legs would be useless, since long ones would interfere with their motion, and short ones could 
not move their long bodies. Since the plan of organization limits the snakes to only four $\operatorname{legs}$, and since this number would be useless, they have disappeared.

Many insects are destitute of wings, although wings are a part of the plan of organization of this group. They are absent only in those forms whose habits render wings useless, consequently they have disappeared through disuse.

The preceding cases are those in which the disuse of an organ has led to its degeneration. The following cases are cited to show that by use an organ increases in size. The formation of the web in the feet of water-bircls has already been given as a character which Lamarck supposes to have been acquired through use; also the case of shorebirds, which, by an effort to elongate their legs, have actually made them so in the course of time. The necks of waterbirds are also long on account of their having been stretched in the efforts to catch fish. The long tongues of the ant-eater, of the woodpecker, and of humming-birds are the result of use, and the long, forked tongue of serpents has come from their using their tongue to feel objects in front of them.

Fishes that have acquired the habit of living in shallow water, flounders, soles, etc., have been forced to swim on their sides in order to approach nearer to the shore. Since more light comes from above than from below, the eye on the under side, straining to turn to the light, has finally migrated to the upper side.

The habit of eating great quantities of food, which distends the digestive organs, has caused the bodies of herbivorous quadrupeds to become large, as seen in the elephant, the rhinoceros, oxen, horses, and buffaloes. The habit of standing for a long time on their feet has caused some animals to develop hard, thick hoofs. Herbivorous animals, that in habit countries where they are constantly subjected to attack, as deer and antelopes for example, are forced to escape by rapid flight, and in consequence their bodies have become slen- 
derer and their legs thinner. The horns, antlers, and protuberances that many of these animals possess are the results of their butting each other when angered.

"The long neck and the form of the giraffe offer a curious case. We know that the giraffe is the tallest of all animals. It inhabits the centre of Africa, living in those localities where the earth is nearly always dry and without herbage. It is obliged to browse on the foliage of trees, and this leads to its stretching continually upwards. As a result of this habit, carried on for a long time, in all the individuals of the race, the anterior limbs have become longer than the posterior, and its neck has also lengthened, so that the giraffe without rising on its hind-legs stretches up its neck and can reach to the height of six metres."

The curved claws of the carnivora have arisen from the necessity of grasping their prey. The power of retracting the claws has also been acquired by the effort to draw them in when running over hard ground. The abdominal pouch of the kangaroo, in which the young are carried, opens anteriorly, and this has led to the animal standing erect so that its young are not injured. In consequence, the fore-legs have become shorter through disuse, and the hind-legs have become stronger through use. The tail, which is also used as a support, has become enormously thick at its base.

The sloth has been compelled to seek refuge in the trees, and has taken up its abode permanently there, feeding on leaves. Its movements are limited to those involved in crawling along the limbs in order to reach the leaves. After feeding it remains inactive and sluggish, these habits being provoked by the heat of the climate. The results of its mode of life have been to cause the arms to become elongated due to the habit of the sloth of grasping the limbs of the tree; the claws of the fingers and toes have also become long and hooked in order to retain their hold. The digits that do not make any individual movements have lost the power to do 
so, and have become fused, and can only be bent in and straightened out. The thighs, being bent out to clasp the larger branches, have caused the pelvis to widen, and, in consequence, the cotyloid cavities have become directed backward. Many of the bones of the skeleton have become fused, as a result of the immobility of the animal.

Lamarck says, that "Nature, in producing, successively, all the species of animals, beginning with the most imperfect, or the most simple, and terminating with the most perfect, has gradually complicated their organization. These animals becoming scattered throughout the habitable regions of the globe each species has receired from the influences of its surroundings its present habits, and the modifications of the parts the use of which we recognize."

Such are Lamarck's views and a fairly complete statement of the facts from which he draws his conclusions. His illustrations appear naïve, and often not a little ludicrous, but it must be admitted that, despite their absurdities, his theory appears in some cases to account wonderfully well for the facts. The long legs of wading birds, the long neck and disproportionately long fore-legs of the giraffe, the structure of the sloth, and particularly the degeneration of the eyes of animals living in the dark, seem to find a simple explanation in the principle of the inheritance of acquired characters. But the crucial point of the entire theory is passed over in silence, or rather is taken for granted by Lamarck, namely, the inheritance in the offspring of the characters acquired through use or disuse in the parent. He cloes not even discuss this topic, but in several places states unreservedly that the increase or decrease of a part reappears in the next generation. It is here that Lamarck's theory has been attacked in more. modern times, for as soon as experimental proof was demanded to show that the results of use or of clisuse of an organ is inherited, no such proof was forthcoming. Yet the theory is one that has the great merit of being capable of 
experimental test, and it is astonishing to find that, with the immense amount that has been written by his followers, so few attempts have been made to give the theory a thorough test. The few results that have been obtained are not, however, favorable to the theory, but almost the only attempts at experiment that have been made in this direction have been those of mutilating certain parts; and were it not for popular belief to the effect that such mutilations are inherited, one would least expect to get evidence for or against the theory in this direction. Lamarck himself believed that the changes were slowly acquired, and I think modern Lamarckians are justified in claiming that the validity of the theory can only be tested by experiments in which the organism is subjected to influences extending over a considerable period, although Lamarck appears to have believed that the first results may appear quite soon. Before expressing any opinion in regard to the probability of the theory, let us examine what the followers of Lamarck have contributed in the way of evidence to the theory, rather than the applications that they have made of the theory. We shall also find it profitable to consider some of the modern criticism, to which the theory has been subjected.

Despite the contempt with which Darwin referred to Lamarck's theory, he himself, as we have seen, often made use of the principle of the inheritance of acquired characters, and even employed the same illustrations cited by Lamarck. Darwin seems to have misunderstood Lamarck's view, and to have accepted the current opinion that Lamarck supposed an animal acquired a new organ by desiring or needing it. Darwin says, "Heaven forefend me from Lamarck's nonsense of a tendency to progressive adaptation from the slow willing of the animals." Darwin speaks of Lamarck as stating that animals will that the egg shall be a particular form so as to become attached to particular objects. Lamarck's latest biographer, Packard, says he is 
unable to find any statements of this sort in Lamarck's writings.

The following cases that Darwin tried to explain through the inheritance of acquired characters are exactly like those to which Lamarck applied his theory. The bones of the wing of the domestic duck weigh less than those of the wild duck, and the bones of the leg more. Darwin believes this is due to the effects of the inheritance of acquired characters. The drooping ears of many domestic mammals are also explained by him as a result of disuse - "the animals being scldom much alarmed." In speaking of the male of the beetle, Onites apelles, Darwin quotes Kirby to the effect that the tarsi are so habitually lost that the species has been described without this part of the foot. In the sacred beetle of Egypt the tarsus is totally absent. Hence he concludes that the absence of tarsi in the sacred beetle, and the rudimentary condition of the tarsus in others, is probably the result of disuse, rather than a case of inheritance of a mutilation. Darwin grants that "the evidence that accidental mutilations can be inherited is at present not decisive, but the remarkable case observed by Brown-Séquard in guineapigs of the inherited effects of operations should make us cautious in denying this tendency."

The wingless condition of several insects inhabiting oceanic islands has come about, Darwin thinks, through disuse. The ostrich also, owing to its increase in size, made less use of its wings and more use of its legs, with the result that its wings degenerated and its legs got stronger. The rudimentary condition of the eyes of the mole is the result of clisuse, "aided perhaps by natural selection." Many of the animals inhabiting the cares of Kentucky and of Carniola are blind, and this is ascribed to disuse. "As it is difficult to imagine that the eyes, though useless, could be in any way injurious to animals living in darkness, their loss may be attributed to disuse." The long neck of the giraffe 
Darwin attributes partly to natural selection and partly to use.

These references will suffice to show that Darwin is in full accord with the main argument of Lamarck. In fact, the curious hypothesis of pangenesis that Darwin advanced was invented partly to account for the inheritance of acquired characters. Despite the hesitancy that Darwin himself felt in advancing this view, and contrary to Huxley's advice, he at last published his provisional hypothesis of pangenesis in the twenty-seventh chapter of his "Animals and Plants under Domestication."

\section{Darwin's Hypothesis of Pangenesis}

The study of bud variation, of the various forms of inheritance, and of reproduction and of the causes of variation, led him, Darwin says, to the belief that these subjects stand in some sort of relation to each other. He says: "I have been led, or rather forced, to form a view which to a certain extent connects these facts by a tangible method. Every one would wish to explain to himself, even in an imperfect manner, how it is possible for a character possessed by some remote ancestor suddenly to reappear in the offspring; how the effects of increased or decreased use of a limb can be transmitted to the child; how the male sexual element can act not solely on the ovules, but occasionally on the mother form; how a hybrid can be produced by the union of the cellular tissue of two plants independently of the organs of generation; how a limb can be reproduced on the exact line of amputation, with neither too much nor too little added; how the same organism may be produced by such widely different processes, as budding and true seminal generation; and, lastly, how of two allied forms, one passes in the course of its development through the most complex metamorphoses, and the other does not do so, though when mature both are 
alike in every detail of structure. I am aware that my view is merely a provisional hypothesis or speculation; but, until a better one be advanced, it will serve to bring together a multitude of facts which are at present left disconnected by any efficient cause."

In presenting the hypothesis of pangenesis Darwin begins by enumerating the different kinds of sexual and asexual processes of reproduction, for which he hopes to offer a provisional explanation. Here we find mentioned various methods of budding and self-division, regeneration, parthenogenesis, sexual reproduction, and the inheritance of acquired characters. It is with the last only that we are here chiefly concerned; in fact, the need of an hypothesis of this sort to explain the other kinds of inheritance is by no means evident. There are, however, two other phenomena, besides that of the supposed inheritance of acquired characters, to which the hypothesis of pangenesis might appear to apply specially, namely, the effect of foreign pollen on the tissues of the mother plant, and the supposed influence of the union with the first male on the subsequent young (telegony). It is, however, far from being shown that any influence of this latter kind really occurs, despite the fact that it is generally believed in by breeders.

It is important to observe that Darwin proposes to explain on the hypothesis of pangenesis, not only the inheritance of characters acquired through use, but also the decrease of structures through disuse; and this applies, not only to the structure, but to function as well, as when the intelligence of the dog is explained through his association with man, and the tameness of the domestic rabbits through their long confinement. In the following quotation these points are referred to: "How can the use or clisuse of a particular limb or of the brain affect a small aggregate of reproductive cells, seated in a distant part of the body, in such a manner that the being developed from these cells inherits the characters of either 
one or both parents? Even an imperfect answer to this question would be satisfactory."

Coming now to the theory, we find that it consists of one chief assumption and several minor ones. "It is universally admitted that the cells or units of the body increase by selfdivision or proliferation, retaining the same nature, and that they ultimately become converted into the various tissues and substances of the body. But besides this means of increase I assume that the units throw off minute granules which are dispersed throughout the whole system; that these, when supplied with proper nutriment, multiply by self-division, and are ultimately developed into units like those from which they were originally derived. These granules may be called gemmules. They are collected from all parts of the system to constitute the sexual elements, and their development in the next generation forms a new being; but they are likewise capable of transmission in a dormant state to future generations, and may then be developed. . . . Gemmules are supposed to be thrown off by every unit, not only during the adult state, but during each stage of development of every organism; but not necessarily during the continued existence of the same unit. Lastly, I assume that the gemmules in their dormant state have a mutual affinity for each other, leading to their aggregation into buds, or into the sexual elements. Hence, it is not the reproductive organs, or buds, which generate new organisms, but the units of which each individual is composed. These assumptions constitute the provisional hypothesis which I have called Pangenesis."

It will be noticed that the first assumption is that the cells throw off minute gemmules or granules. The second assumption is that these are collected in the reproductive organs, or in buds, or in regenerating parts; the third assumption is that the gemmules may lie dormant through several generations; the fourth, that the development of the reproductive cells is not so much the development of the cell itself, but of 
the gemmules that have collected in it. The fifth assumption is that the gemmules are thrown off at all stages of development; the sixth, that in their dormant state they have a mutual affinity for each other; the seventh, that there may be a sort of continual competition in the germ-cells between the original gemmules and the new ones, and, according to which win, the old or the new form develops. Thus we see on closer analysis that the pangenesis hypothesis is made up of a goodly number of different assumptions. At least half a dozen imaginary properties are ascribed to the imaginary gemmules, and these attributes are all essential to the working of the hypothesis.

Some of the more obvious objections to the hypothesis have been stated by Darwin himself. Such, for instance, as our ignorance at what stage in their history the body-cells are capable of throwing off gemmules, and whether they collect only at certain times in the reproductive organs, as the increased flow of blood to these organs at certain seasons might seem to indicate. Nor have we any evidence that they are carried by the blood at all. The experiment of Galton, of transfusing the blood of one animal into another, and finding that this produced no effect on the young that were born later, might be interpreted to mean that gemmules are not transported by the blood; but this kind of experiment is inconclusive, especially in the light of recent results on the effect of the blood of one animal on that of another.

A part of the evidence on which Darwin relied to support his theory has been shown to be incorrect by later work. Thus the assumption that more than a single pollen grain, or more than one spermatozoon, is necessary in some cases for fertilization, is certainly wrong. In most cases, in fact, the entrance of more than one spermatozoon into the egg is disastrous to the development. The cases referred to by Darwin can probably be explained by the difficulty that some of the pollen grains, or spermatozoa, may have in penetrating 
the egg, or to the immaturity or impotence of some of the male germ-cells, and not to the need of more than one to accomplish the true fertilization.

Darwin's idea that the small number of gemmules in the unfertilized egg may account for the lack of power of such eggs to develop until they are fertilized, has been, shown to be incorrect by recent results in experimental embryology. We now know that many different kinds of stimuli have the power to start the development of the egg. Moreover, we also know that if a single spermatozoon is supplied with a piece of egg-protoplasm without a nucleus, it suffices to cause this piece of protoplasm to develop.

In the case of regeneration, which Darwin also tries to explain on the pangenesis hypothesis, we find that there is no need at all for an hypothesis of this sort; and there are a number of facts in connection with regeneration that are not in harmony with the hypothesis. For instance, when a part is cut off, the same part is regenerated; but under these circumstances it cannot be imagined that the part removed supplies the gemmules for the new part. Darwin tries to meet this objection by the assumption that every part of the body contains gemmules from every other part. But it has been shown that if a limb of the newt is completely extirpated, a new limb does not regenerate; and there is no reason why it should not do so on Darwin's assumption that germs of the limb exist throughout the body.

The best-authenticated cases of the influence of the male on the tissues of the female are those in plants, where one species, or variety, is fertilized by another. Thus, if the orange is fertilized by the pollen of the lemon, the fruit may have the color and flavor of the lemon. Now the fruit is a product of the tissues of the ovary of the female, and not a part of the seedling that develops in the fruit from the crossfertilized egg-cell. Analogous cases are recorded for the bean, whose pods may have their color influenced by fertil- 
izing the flower with pollen of another variety having pods of a different color. In these cases we do not know whether the color of the fruit is influenced directly by the foreign pollen, or whether the influence is through the embryo that develops from the egg-cell. The action may appear to be the same, however, in either case; but because it seems probable here that there is some sort of influence of one tissue on another, let us not too readily conclude that this is brought about through any such imaginary bodies as gemmules. It may be directly caused, for instance, by some chemical substance produced in the young hybrid plant. If this is the case, the result would not be different in kind from that of certain flowers whose color may be influenced by certain chemical substances in the soil.

In the cases amongst animais, where the maternal tissues are believed to be influenced by a previous union with the male, as in the oft-cited case of Lord Morton's mare, a reëxamination of the evidence by Ewart has shown that the case is not demonstrated, and not even probable. Several years ago I tried to test this view in the case of mice. A white mouse was first bred to a dark male house-mouse, and the next time to a white mouse, but none of the offspring from the second union showed any trace of black. If the spermatozoa of the dark mouse are hypodermically injected into the body-cavity of the female, the subsequent young from a white male show no evidence that the male cells have had any influence on the ovary.

The following facts, spoken of by Darwin himself, are not in favor of his hypothesis of pangenesis: "But it appears at first sight a fatal objection to our hypothesis that a part of an organ may be removed during sereral successive generations, and if the operation be not followed by clisease, the lost part reappears in the offspring. Dogs and horses formerly had their tails docked during many generations without any inherited effect; although, as we have seen, there is some reason to believe that the tailless condi. 
tions of certain sheep-dogs is clue to such inheritance." The answer that Darwin gives is that the gemmules themselves, that were once derived from the part, are still present in other parts of the body, and it is from these that the organs in the next generation may be derived. But Darwin fails to point out that, if this were the case, it must also be true for those cases in which an organ is no longer used. Its decrease in size in successive generations cannot be due to its disuse, for the rest of the body would supply the necessary gemmules to keep it at its full state of development. Thus, in trying to meet an obvious objection to his hypothesis, Darwin brings forward a new view that is fatal to another part of his hypothesis.

The following cases, also given by Darwin, are admitted by him to be inexplicable on his hypothesis: "With respect to variations due to reversion, there is a similar difference between plants propagated from buds and seeds. Many varieties can be propagated securely by buds, but generally or invariably revert to their parent forms by seed. So, also, hybridized plants can be multiplied to any extent by buds, but are continually liable to reversion by seed, - that is, to the loss of their hybrid or intermediate character. I can offer no satisfactory explanation of these facts. Plants with variegated leaves, phloxes with striped flowers, barberries with seedless fruit, can all be securely propagated by buds taken from the stem or branches; but buds from the roots of these plants almost invariably lose their character and revert to their former condition. This latter fact is also inexplicable, unless buds developed from the roots are as distinct from those on the stem, as is one bud on the stem from another, and we know that these latter behave like independent organisms.". As Darwin here states, these facts appear to be directly contradictory to his hypothesis, and he makes no effort to account for them.

The entire question of the possibility of the inheritance of acquired characters is itself at present far from being on a 
satisfactory basis, as we shall try to show; and Darwin's attempt at an explanation, in his chapter on pangenesis, does not put the matter in a much more satisfactory condition.

\section{The Neo-Lamarckian School}

Let us now turn our attention to a school that has grown up in modern times, the members of which call themselves Neo-Lamarckians. Let us see if they have supplied the essential evidence that is required to establish the Lamarckian view, namely, that characters acquired by the individual are transmitted to the offspring.

Lamarck's views were adopted by Herbert Spencer, and play an important rôle in his "Principles of Biology" ( I 866I $87 \mathrm{I}$ ), and even a more conspicuous part in his later writings. In the former he cites, amongst other cases, that of " a puppy taken from its mother at six weeks old who, although never taught 'to beg' (an accomplishment his mother had been taught), spontaneously took to begging for everything he wanted when about seven or eight months old." If tricks like this are inheritable is it not surprising that more puppies do not stand on their hind-legs?

The larger hands of the laboring classes in England are supposed to be inherited by their children, and the smaller hands of the leisure classes are supposed to be the result of the disuse of the hands by their ancestors; but even if these statements in regard to size are true, there are many other conceivable causes that may have led to this result.

Short-sightedness appears more often, it is said, in those classes of society that make most use of their eyes in reading and in writing; but if we ask for experimental eridence to show that this is due to inheritance, and not due to the children spoiling their eyes at school, there is none forthcoming. The problem is by no means so simple as the uninitiated may be led to believe. 
Spencer thinks that "some of the best illustrations of functional heredity are furnished by mental characteristics." $\mathrm{He}$ cites the musical faculty as one that could not have been acquired by natural selection, and must have arisen through the inheritance of acquired modifications. The explanation offered is "that the habitual association of certain cadences of speech with certain emotions has clearly established in the race an organized and inherited connection between such cadences and such emotions, . . . and that by the continued hearing and practice of melody there has been gained and transmitted an increasing musical sensibility." But a statement that the results have been acquired in this way does not supply the proof which the theory is in need of ; neither does it follow that, because the results cannot be explained by the theory of natural selection, therefore, they must be explained by the Lamarckian theory.

The clearest proofs that Spencer finds of the inheritance of acquired characters are in the well-known experiments of Brown-Séquard. These experiments will be more fully discussed below. Amongst the other morbid processes that Spencer thinks furnish evidence in favor of this view, are cases of a tendency to gout, the occurrence of mental tricks, musical prodigies, liability to consumption, in all of which cases the fundamental distinction between the inheritance of an acquired character and the inherited tendency toward a particular malady is totally ignored.

Twenty-seven years later (in 1893) Spencer took up the open challenge of the anti-Lamarckian writers, and by bringing forward a number of new arguments attempted to reinstate the principle of the inheritance of acquired characters. His first illustration is drawn from the distribution of the sense of touch in different parts of our bodies. Weber's experiments have shown that if the sharp points of a pair of compasses are applied to the tips of the forefingers, the sensation of two separate points is given when the points are 
only one-twelfth of an inch apart, and if the points are moved nearer together, they give the sensation of only one point. The inner surfaces of the second joints of the fingers can only distinguish two points when they are one-sixth of an inch apart. The innermost joints are less discriminating, and are about equal in the power of discrimination to the tip of the nose. The end of the big toe, the palm of the hand, and the cheek discriminate only about one-fifth as well as do the tips of the fingers. The back of the hand and the top of the head distinguish only about one-fifteenth as well as the finger-tips. The front of the thigh, near the knee, is somewhat less sensitive than the back of the hand. On the breast the points of the compasses must be separated by more than an inch and a half in order to give two sensations. In the middle of the back the points must be separated by two and a half inches, or more, in order to give two separate impressions.

What is the meaning of these differences, Spencer asks.* If natural selection has brought about the result, then it must be shown that "these degrees of endowment have advantaged the possessor to such an extent that not infrequently life has been directly or indirectly preserved by it." He asks if this, or anything approaching this, result could have occurred.

That the superior perceptiveness of the forefinger-tip might have arisen through selection is admitted by Spencer, but how could this have been the case, he asks, for the middle of the back, and for the face? The tip of the nose has three times more power of discrimination than the lower part of the forehead. Why should the front of the thigh near the knee be twice as perceptive as in the middle of the thigh; and why should the middle of the back and of the neck and the middle of the forearm and of the thigh stand at such low levels? Is it possible, Spencer asks again, that natural selection has determined these relations, and 
if not, how can they be explained? His reply is that the differences can all be accounted for on the theory of the inheritance of use, for it is evident that "these gradations in tactile perceptiveness correspond with the gradations in the tactual exercise of the parts." Except from contact with the clothing the body receives hardly any touch sensations from outside, and this accounts for its small power of discrimination. The greater sensitiveness of the chest and abdomen, as compared with the back, is due to these regions being more frequently touched by the hands, and is also owing to inheritance from more remote ancestors, in which the lower surface of the body was more likely to have come in contact with foreign objects than was the back. The middle of the forearm and of the thigh are also less exposed than the knee and the hand, and have correspondingly the power of tactile discrimination less well developed.

Weber showed that the tip of the tongue is more sensitive than any other part of the body, for it can distinguish between two points only one twenty-fourth of an inch apart. Obviously, Spencer says, natural selection cannot account for such extreme delicacy of touch, because, even if it were useful for the tongue to distinguish objects by touch, this power could never be of vital importance to the animal. It cannot even be supposed that such delicacy is necessary for the power of speech.

The sensitiveness of the tongue can be accounted for, however, Spencer claims, as the result of the constant use of the tongue in exploring the cavity of the mouth. It is continually moving about, and touching now one part, and now another, of the mouth cavity. "No advantage is gained. It is simply that the tongue's position renders perpetual exploration almost inevitable." No other explanation of the facts seemed possible to Spencer.

Two questions will at once suggest themselves. First, can it be shown that the sensitiveness to touch in various parts of 
the body is the result of individual experience? Have we learned to discriminate in those parts of the body that are most often brought into contact with surrounding objects? Even the power of discrimination in the tips of the fingers can be improved, as Spencer himself has shown, in the case of the blind, and of skilled compositors. Can we account in this way for the power of discrimination in various parts of the body? In other words, if, beginning in infancy, the middle of the back constantly came into contact with surrounding objects, would this region become as sensitive as the tips of the fingers? The experiment has not, of course, been carried out, but it is not probable that it would succeed. I venture this opinion on the ground of the relative number of the nerves and of the organs of touch on the back, as compared with those of the finger-tips. But, it will be asked, will not the number of the sense-organs become greater if a part is continually used by the individual? It is improbable that much improvement could be brought about in this way. The improvement that takes place through experience is probably not so much the result of the development of more senseorgans, as of better discrimination in the sensation, because the increased power can be very quickly acquired.

An examination of the relative abundance of touch-spots in the skin shows that they are much more numerous in regions of greater sensitiveness. The following table, taken from Sherrington's account of sense-organs in Schaefer's "Textbook of Physiology," gives the smallest distance that two points, simultaneously applied, can be recognized as such (and not simply as one impression) in different regions.

Tip of tongue.

Volar surface of ungual phalanx of finger $\quad . \quad . \quad . \quad 2.3$

Red surface of lip . . . . . . . . . . . . 4.5

Volar face of second phalanx . . . . . . . . . . 4.5

Dorsal face of third phalanx . . . . . . . . . . 6.8

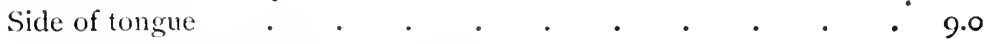

Third line of tongue, $27 \mathrm{~mm}$. from tip . . . . . . 9.0 
Plantar face of ungual phalanx of first toe . . . . . II.3

Palm

Back of second phalanx of finger

Forehead

Back of ankle

Back of hand .

Forearm, leg.

Dorsum of foot

Outer sternum

Back of neck .

Middle of back

Upper arm, thigh

The great difference in the sensitiveness of the skin in the different regions is very striking, and if, as seems probable, about the same proportionate difference is found at birth, then the degree of sensibility of the different regions is inborn, and is not the result of each individual experience. Until it can be shown that more of the sense-organs develop in any special part, as the result of the increased use of the part, we have no real basis on which to establish, even as probable, the Lamarckian view.

But, after all, is the distribution of the sense-organs exactly that which we should expect on the Lamarckian theory? Has not Spencer taken too much for granted in this direction? The lower part of the forearm (represented by I 5 ) we should expect to be more sensitive than the protected surface of the eyclid (II.3), but this is not the case. The forehead (22.6) is much less sensitive than the forearm, and only half as sensitive as the eyelid. The knee (36.I) is still less sensitive than any of these other parts, and this does not in the least accord with the theory, since in its constant moving forward it must be continually coming in to contact with foreign bodies. The fact that the back is as insensitive as the upper arm (67.7) can hardly be accredited in favor of the theory. The great difference between the lower third of the forearm on the ulnar surface (I5) and the upper arm (67.7) seems 
out of all proportion to what we should expect on the theory. And is it not a little odd that the end of the nose should be so highly sensitive?

There is another point that we cannot afford to neglect in this connection. It is known that in addition to touch-spots there are warm and cold spots in the skin, which produce, when touched, the sensation of warmth, or of cold, respectively, and not the sensation of touch. The degree of sensitiveness of different regions of the body throws an interesting side-light on Spencer's argument.

The warm spots are much fewer than the cold spots. The spots are arranged in short lines radiating from centres which coincide with hairs. The number of these spots varies a good deal, even in the same region of the skin. If the sensitiveness of the skin is tested, the following results will be obtained. The list includes twelve grades of sensitiveness, beginning with the places giving the lowest maximum of intensity. About one hundred square areas were tested in each region.

\section{COLD SENSATIONS}

I. Tips of fingers and toes, malleoli, ankle.

2. Other parts of digits, tip of nose, olecranon.

3. Clabella, chin, palm, gums.

4. Occiput, patella, wrist.

5. Clavicle, neck, forehearl, tongue.

6. Buttocks, upper eyelid.

7. Lower eyelid, popliteal space, sole, cheek.

8. Inner aspect of thigh, arm above elbow.

9. The intercostal spaces along axillary line.

Io. Nammary areola.

I I. Nipple, flank.

12. Certain areas of the loins and abdomen.

\section{WARMTH SENSATIONS}

o. Lower gum, mucosa of cheek, cornea.

I. Tips of fingers and toes, cavity of mouth, conjunctiva, and patella. 
2. Remaining surface of digits, middle of forehead, olecranon.

3. Glabella, chin, clavicle.

4. Palm, buttock, popliteal space.

5. Neck.

6. Back.

7. Lower eyelid, cheek.

8. Nipple, loin.

These two tables show the great differences in the range of sensitiveness to cold and to warmth in different parts of the body. I doubt if any one will attempt to show that these differences of range of sensation can be accounted for either by natural selection or by the Lamarckian hypothesis.

Of course, it does not necessarily follow that, because this is true for the warm and cold spots, that it must also be true for the tactile organs; but I think that the fact of such a great difference in the responsiveness to cold and to warmth in different parts of the body should put us on our guard against a too ready acceptation of Spencer's argument. More especially is this seen to be necessary, when, as has been shown above, the distribution of the touch-organs themselves by no means closely corresponds to what we should expect, if they have developed in response to contact, as Spencer maintains.

The other main argument advanced by Spencer to fortify the theory of the inheritance of acquired characters, and at the same time to show the inadequacy of the theory of natural selection, is based on the idea of what he calls the "cooperation of the parts" that is required in order to carry out any special act. Spencer contends that "the relative powers of coöperative parts cannot be adjusted solely by the survival of the fittest, and especially where the parts are numerous and the coöperation complex."

Spencer illustrates his point by the case of the extinct Irish elk, whose immensely developed horns weighed over a hundredweight. The horns, together with the massive skull, could not have been supported by the outstretched 
neck without many and great changes of the muscles and bones of the neck and of the fore-part of the body. Unless, for instance, the fore-legs had been also strengthened, there would be failure in fighting and in locomotion. Since "we cannot assume spontaneous increase of all these parts proportionate to the additional strains, we cannot suppose them to increase by variations one at once, without supposing the creature to be disadvantaged by the weight and nutrition of the parts that were for a time useless, - parts, moreover, which would revert to their original sizes before the other needful variations occurred."

The answer made to this argument was that coördinating parts vary together. In reply to which Spencer points to the following eases, which show that this is not so: The blind crayfish in the Kentucky caves have lost their eyes, but not the stalks that carry them. Again, the normal relation between the length of tongue and of beak in some varieties of pigeons is lost. The greater decrease in the jaws in some species of pet dogs than of the number of their teeth has eaused the teeth to become crowded." "I then argued that if coöperative parts, small in number, and so closely associated as these are, do not vary together, it is unwarrantable to allege that coöperative parts, which are very numerous and remote from one another, vary together." Spencer puts himself here into the position of seriously maintaining that, because some coöperative parts do not vary together, therefore no coöperative parts have ever done so, and he has taken this position in the face of some well-known cases in which certain parts have been found to vary together.

In this same connection Spencer brings up the familiar piece de résistance of the Lamarckian school, the giraffe. He recognizes that the chief traits in the structure of this animal are the result of natural selection, since its efforts to reach

1 It is curious that spencer does not see that this case is as much against his point as in favor of it, since the unused teeth did not also degenerate. 
higher branches could not be the cause of the lengthening of the legs. But "the coadaptation of the parts, required to make the giraffe's structure useful, is much greater than at first appears." For example, the bones and the muscles of the hind-legs have been also altered, and Spencer argues that it. is "impossible to believe" that all parts of the hind-quarters could have been coadapted to one another, and to all parts of the fore-quarters. A lack of coadaptation of a single muscle "would cause fatal results when high speed had to be maintained while escaping from an enemy."

Spencer claims that, since I886, when he first published this argument, nothing like an adequate response has been made; and I think he might have added that an adequate answer is not likely to be forthcoming, since nothing short of a demonstration of how the giraffe really evolved is likely to be considered as sufficient. Wallace's reply, that the changes in question could have been brought about by natural selection, since similar changes have been brought about by artificial selection, is regarded as inadequate by Spencer, since it assumes a parallel which does not exist. Nevertheless, Wallace's reply contains, in my opinion, the kernel of the explanation, in so far as it assumes that congenital variation ${ }^{1}$ may suffice to account for the origin of a form even as bizarre as that of the giraffe. The ancon ram and the turnspit dog were marked departures from the normal types, and yet their parts were sufficiently coördinated for them to carry out the usual modes of progression. It would not have been difficult, if we adopted Spencer's mode of arguing, to show that these new forms could not possibly have arisen as the result of congenital variations.

Again, it might be argued that the large, powerful drayhorse could not have arisen through a series of variations from the ordinary horse, because, even if variations in the

1 Wallace assumes fluctuating variation to suffice, but in this I cannot agree with him. 
right direction occurred in the fore-quarters, it is unlikely that similar variations would occur in the hind-quarters, etc. Yet the feat has been accomplished, and while it is difficult to prove that the inheritance of acquired characters has not had a hand in the process, it is improbable that this has been the case, but rather that artificial selection of some kind of variations has been the factor at work.

So long as the Lamarckian theory is supported by arguments like these, it can never hope to be established with anything more than a certain degree of probability. If it is correct, then its demonstration must come from experiment. This brings us to a consideration of the experimental evidence which has been supposed by some writers to give conclusive proof of the validity of the theory.

The best direct evidence in favor of the Lamarckian argument is that furnished by the experiments of Brown-Séquard. He found, as the result of injury to the nervous system of guinea-pigs, that epilepsy appeared in the adult animal, and that young born from these epileptic parents became also epileptic. Still more important was his discovery that, after an operation on the nerves, as a result of which certain organs, the ear or the leg, for instance, are affected, the same affection appears in the young born from such parents. These results of Brown-Séquard have been vouched for by two of his assistants, and his results in regard to the inheritance of epilepsy have been confirmed by Obersteiner, and by Luciani on dogs. Equally important is their later confirmation, as far as the main facts go, by Romanes.

Brown-Séquard gives the following summary of his results. I follow Romanes' translation in his book on "Darwin and after Darwin," where there is also given a careful analysis of Brown-Séquard's results, as well as the outcome of the experiments of Romanes himself. The summary is as follows :-

I. "Appearance of epilepsy in animals born of parents which had been rendered epileptic by an injury to the spinal cord. 
2. Appearance of epilepsy also in animals born of parents which had been rendered epileptic by section of the sciatic nerve.

3. A change in the shape of the ear in animals born of parents in which such a change was the effect of a division of the cervical sympathetic nerve.

4. Partial closure of the eyelids in animals born of parents in which that state of the eyelids had been caused either by section of the cervical sympathetic nerve, or the removal of the superior cervical ganglion.

5. Exophthalnia in animals born of parents in which an injury to the restiform body had produced that protrusion of the eyeball. This interesting fact I have witnessed a good many times, and seen the transmission of the morbid state of the eye continue through four generations. In these animals modified by heredity, the two eyes generally protruded, although in the parents usually only one showed exophthalmia, the lesion having been made in most cases only on one of the corpora restiformia.

6. Hrematoma and dry gangrene of the ears in animals born of parents in which these ear alterations had been caused by an injury to the restiform body near the nib of the calamus.

7. Absence of two toes out of the three of the hind-leg, and sometimes of the three, in animals whose parents had eaten up their hind-leg toes, which had become anæsthetic from a section of the sciatic nerve alone, or of that nerve and also of the crural. Sometimes, instead of complete absence of the toes, only a part of one or two or three was missing in the young, although in the parent not only the toes, but the whole foot was absent (partly eaten off, partly destroyed by inflammation, ulceration, or gangrene).

8. Appearance of various morbid states of the skin and hair of the neck and face in animals born of parents having had similar alterations in the same parts as effects of an injury to the sciatic nerve." 
Romanes, who later went over the same ground, in part under the immediate direction of Brown-Séquard himself, has made some important observations in regard to these results, many of which he was able to confirm.

He did not repeat the experiment of cutting the cord, but he found that, to produce epilepsy, it was only necessary to cut the sciatic nerve. The "epileptiform habit" does not appear in the animal until some time after the operation; it lasts for some weeks or months, and then disappears. The attacks are not brought on spontaneously, but by "irritating a small area of the skin behind the ear on the same side of the body as that on which the sciatic nerve had been divided." The attack lasts for only a few minutes, and during it the animal is convulsed and unconscious. Romanes thinks that the injury to the sciatic nerve, or to the spinal cord, produces some sort of a change in the cerebral centres, "and that it is this change - whatever it is, and in whatever part of the brain it takes place - which causes the remarkable phenomena in question."

In regard to Brown-Séquard's statements, made in the $3 \mathrm{~d}$ and the $4^{\text {th }}$ paragraphs, in respect to the results of the operation of cutting the cervical sympathetic, Romanes had not confirmed the results when his manuscript went to press; but soon afterward, after Romanes' cleath, a note was printed in Noture by Dr. Hill, announcing that two guinea-pigs from Romanes' experiment had been born, "both of which exhibited a well-marked droop of the upper eyelid. These guinea-pigs were the offspring of a male and female in both of which I had produced for Dr. Romanes, some months earlier, a droop of the left upper eyelid by division of the left cervical sympathetic nerve. This result is a corroboration of the series of Brown-Séquard experiments on the inheritance of aequired characters."

Romanes states that he also found that injury to a particular spot of the restiform bodies is quickly followed by a 
protrusion of the eye on the same side, and further, that he had "also had many cases in which some of the progeny of parents thus affected have shown considerable protrusion of the eyeballs of both sides, and this seemingly abnormal protrusion has occasionally been transmitted to the next generation. Nevertheless, I am far from satisfied that this latter fact is anything more than an accidental coincidence." This reservation is made on the ground that the protrusion in the young is never so great as in the parents, and also because there is amongst guinea-pigs a considerable amount of individual variation in the degree of prominence of the eyeballs. Romanes, while unwilling to deny that an "obviously abnormal amount of protrusion, due to the operation, may be inherited in lesser degree," is also unwilling to affirm so important a conclusion on the basis of these experiments alone.

In regard to Brown-Séquard's 6th statement, Romanes found after injury to the restiform body that hæmatoma and dry gangrene may supervene, either several weeks after the operation, or at any subsequent time, even many months afterward. The disease usually affects the upper parts of both ears, and may then gradually extend downward until nearly the whole ear is involved. "As regards the progeny of animals thus affected in some cases, but by no means in all, a similarly morbid state of the ears may arise apparently at any time in the life history of the individual. But I have observed that in cases where two or more individuals of the same litter develop this diseased condition, they usually do so at about the same time, even though this may be months after birth, and therefore after the animals are fully grown." Moreover, the morbid process never extends so far in the young as it does in the parents, and "it almost always affects the middle third of the ear." Several of the progeny from this first generation, which had apparently inherited the disease, but had not themselves been directly operated upon, showed a portion of 
the ear consumed apparently by the same disease. Romanes then gives the following significant analysis of this result. Since a different part of the ear of the progeny is affected, and also a "very much less quantity thereof," it might seem that the result was due either to a mere coincidence, or to the transmission of microbes. But he goes on to say, that he fairly well excluded both of these possibilities, for, in the first place, he has never observed "the very peculiar process in the ears, or in any other parts of guinea-pigs which have neither themselves had the restiform bodies injured, nor been born of parents thus mutilated." In regard to microbes, Romanes tried to infect the ears of normal guineapigs by first scarifying these parts, and then rubbing them with the diseased surfaces of the ears of affected guineapigs. In not a single case was the disease produced.

Romanes concludes that these "results in large measure corroborate the statements of Brown-Séquard; and it is only fair to add that he told me they were the results which he had himself obtained most frequently, but that he had also met with many cases where the diseased condition of the ears in parents affected the same parts in their progeny and also occurred in more equal degrees."

We come now to the remarkable conclusion given in Brown-Séquard's 7 th statement, in regard to the absence of toes in animals whose parents had eaten off their own hind toes and even parts of their legs. Romanes got neuroses in the animals operated upon, and found that the toes might be eaten off; but none of the young showed any defect in these parts. Furthermore, Romanes repeated the same operation upon the descendants through six successive generations, so as to produce, if possible, a cumulative effect, but no inheritance of the mutilation was observed. "On the other hand, Brown-Séquard informed me that he had observed this inherited absence of toes only in about one or two per cent of cases." It is possible, therefore, Romanes adds, that his 
own experiments were not sufficiently numerous to have obtained such cases.

In this connection I may give an account of some observations that I made while carrying out some experiments in telegony with mice. I found in one litter of mice that when the young came out of the nest they were tailless. The same thing happened again when the second litter was produced, but this time I made my observations sooner, and examined the young mice immediately after birth. I found that the mother had bitten off, and presumably eaten, the tails of her offspring at the time of birth. Had I been carrying on a series of experiments to see if, when the tails of the parents were cut off, the young inherit the defect, I might have been led into the error of supposing that I had found such a case in these mice. If this idiosyncrasy of the mother had reappeared in any of her descendants, the tails might have disappeared in succeeding generations. This perversion of the maternal instincts is not difficult to understand, when we recall that the female mouse bites off the navel-string of each of her young as they are born, and at the same time eats the afterbirth. Her instinct was carried further in this case, and the projecting tail was also removed.

Is it not possible that something of this sort took place in Brown-Séquard's experiment? The fact that the adults had eaten off their own feet might be brought forward to indicate the possibility of a perverted instinct in this case also. At least my observation shows a possible source of error that must be guarded against in future work on this subject.

In regard to the 8th statement of Brown-Séquard, as to various morbid states of the skin, Romanes did not test this, because the facts which it alleges did not seem of a sufficiently definite character.

These experiments of Brown-Séquard, and of those who have repeated them, may appear to give a brilliant experimental confirmation of the Lamarckian position; yet I think, 
if I were a Lamarckian, I should feel very uncomfortable to have the best evidence in support of the theory come from this source, because there are a number of facts in the results that make them appear as though they might, after all, be the outcome of a transmitted disease, as Weismann claims, rather than the inheritance of an acquired character. Until we know more of the pathology of epilepsy, it may be well not to lay too great emphasis on these experiments. It should not be overlooked that during the long time that the embryo is nourished in the uterus of the mother, there is ample opportunity given for the transmission of material, or possibly even of bacteria. If it should prove true that epilepsy is due to some substance present in the nervous system, such substances could get there during the uterine life of the embryo. Even if this were the case, it may be claimed that it does not give an explanation of the local reappearance of the disease in the offspring. But here also we must be on our guard, for it is possible that only certain regions of the body are susceptible to a given disease; and it has by no means been shown that the local defect itself is inherited, but only the disease. Romanes insists that a very special operation is necessary to bring about certain forms of transmission.

It is well also to keep in mind the fact, that if this sort of effect is inherited, then we must be prepared to accept as a possibility that other kinds of injury to the parent may be transmitted to the offspring. It would be of great disadvantage to animals if they were to inherit the injuries that their parents have suffered in the course of their lives. In fact, we might expect to find many plants and animals born in a dreadful state of mutilation as a result of inheritances of this sort. Thus, while the Lamarckians try to show that, on their principle, characters for the good of the species may be acquired, they must also be prepared, if they accept this kind of evidence, to grant that immense harm may also result from its action. I do not urge this as an argument against 
the theory itself, but point it out simply as one of the consequences of the theory.

It has been shown quite recently, by Charrin, Delamare, and Moussu, that when, after the operation of laparotomy on a pregnant rabbit or guinea-pig, the kidney or the liver has become diseased, the offspring sometimes show similar affections in the corresponding organs (kidney or liver). The result is due, the authors think, to some substance set free from the diseased kidney of the parent that affects the kidney of the young in the uterus. By injecting into the blood of a pregnant animal fresh extracts from the kidney of another animal, the authors believe that the kidney of the young are also affected. It will be observed that this transmission of an acquired character appears to be different from that of transmission through the egg; for it is the developing, or developed organ itself, that is acted upon. The results throw an interesting light on the cases of epilepsy described by Brown-Séquard, since they show that the diseased condition of the parent may be transmitted to the later embryonic stages. May not, therefore, Brown-Séquard's results be also explained as due to direct transmission from the organs of the parent to the similar organs of the young in the uterus?

There is another series of experiments of a different sort that has been used as an argument in favor of the Lamarckian view. These are the results that Cunningham has obtained on young flatfish. He put the very yourg fish, while still bilaterally symmetrical (in which stage the pigment is equally developed on both sides of the body) into aquaria lighted from below. He found that when the young fish begins to undergo its metamorphosis, the pigment gradually disappears on one side, as it would have done under normal conditions, i.e. when they are lighted from above. If, however, the fish are kept for some time longer, lighted from below, the pigment begins to come back again. "The first fact proves that the disappearance of the pigment-cells from 
the lower side in the metamorphosis is an hereditary character, and not a change produced in each individual by the withdrawal of the lower side from the action of light. On the other hand, the experiments show that the absence of pigment-cells from the lower side throughout life is due to the fact that light does not act upon that side, for, when it is allowed to act, pigment-cells appear. It seems to me that the only reasonable conclusion from these facts is, that the disappearance of pigment-cells was originally due to the absence of light, and that the change has now become hereditary. The pigment-cells produced by the action of light on the lower side are in all respects similar to those normally present on the upper side of the fish. If the disappearance of the pigment-cells were due entirely to a variation of the germ-plasm, no external influence could cause them to reappear, and, on the other hand, if there were no hereditary tendency, the coloration of the lower side of the flatfish when exposed would be rapid and complete." 1

This evidence might be convincing were it not weakened by two or three assumptions. In the first place, it is not shown that if the loss of color on the lower side had been the result of the inheritance of an acquired character that the results seen in Cunningham's experiment would follow as a consequence. Thus one of the starting-points of the argument really begs the whole question. In the second place, it is unproven that, had the loss of color of the lower sicle been the result of a variation of the germ-plasm, no external influence could cause it to reappear. In this connection there is another fact that has a bearing on the point here raised. In some species of flatfish the right side is turned down, and in other species the left. Occasionally an individual is found in a right-sided species that is left-sided, and in such cases the color is also reversed. Now, to explain this 
in the way suggested by Cunningham, we should be obliged to assume that some of the ancestors acquired the loss of pigment on one side of the body, and others on the other side according to which side was turned down. This supposition might be appealed to to give us an explanation of the occasional reversal of the symmetry as a rare occurrence at the present time; but the argument is so transparently improbable that, I believe, the Lamarckian school would hesitate to make use of it, yet, in principle, it is about the same as that Cunningham has followed above.

If, on the other hand, we suppose the difference in color of the two sides to have been the result of a germ-variation, we need only suppose that this was of such a kind that the color of the under side is only in a latent condition, and if an external factor can cause a reaction to take place on the light side, it is not surprising that this should call forth the latent color patterns. The result can be given at least a formal explanation on the theory that the original change was a germ-variation.

We come now to the evidence derived from paleontology. A number of evolutionists, more especially of the American school, have tried to show that the evolution of a number of groups can best be accounted for on the theory of the inheritance of acquired characters. A point that we must always bear in mind is that evolution in a direct line need not necessarily be the outcome of Lamarckian factors. Some of our leading paleontologists, Cope, Hyatt, Scott, Osborn, have been strongly impressed by the paleontological evidence in favor of the view that evolution has often been in direct lines; and some, at least, of these investigators have been led to conclude that only the Lamarckian factor of the inheritance of acquired characters can give a sufficient explanation of the facts. Paleontologists have been much impressed by the fact that evolution has been along the lines which we might imagine that it would follow if the effects of use and 
of disuse are inherited. There is, however, no proof that this is the case, although there are a number of instances to which this mode of explanation appears to give the readiest solution. But, as has been said before, it is not this kind of evidence that the theory is in need of, since Lamarck himself gave an ample supply of illustrations. What we need is clear evidence that this sort of inheritance is possible, and, from the very nature of the case, it is just this evidence that fossil remains can never supply.

The same criticism may be made of the work of Ryder, Packard, Dall, Jackson, Eimer, Cunningham, Semper, De Varigny, and others of the Lamarckian school. Despite the large number of cases that they have collected, which appear to them to be most easily explained on the assumption of the inheritance of acquired characters, the proof that such inheritance is possible is not forthcoming. Why not then spend a small part of the energy, that has been used to expound the theory, in demonstrating that such a thing is really possible? One of the chief virtues of the Lamarckian theory is that it is capable of experimental verification or contradiction, and who can be expected to furnish such proof if not the Neo-Lamarckians?

We may fairly sum up our position in regard to the theory of the inheritance of acquired characters in the verdict of "not proven." I am not sure that we should not be justified at present in claiming that the theory is unnecessary and even improbable. 


\section{CHAPTER VIII}

\section{CONTINUOUS AND DISCONTINUOUS VARIATION AND HEREDITY}

THE two terms continuons and discontinuous variation refer to the succession or inheritance of the variations rather than to the actual conditions amongst a group of individuals living at the same time; but this distinction has only a subordinate value. The term fuctuating, or individual iariation, expresses more nearly the conditions of the individuals of a species at any one time, and the continuation of this sort of difference is the continuous variation spoken of above. The discontinuous variations are probably of the same nature as those that have been called mutations, and what Darwin sometimes called sports, or single variations, or definite variations.

\section{Continuous Variation}

If we examine a number of individuals of the same species, we find that no two of them are exactly alike in all particulars. If, however, we arrange them according to some one character, for example, according to the height, we find that there is a gradation more or less perfect from one end of the series to the other. Thus, if we were to take at random a hundred men, and stand them in line arranged according to their height, the tops of their heads, if joined, would form a nearly continuous line; the line will, of course, incline downward from the tallest to the shortest man. This illustrates individual variation. An arrangement of this kind fails to bring out one of the most important facts connected with individual differences. 
If the line is more carefully examined, it will be found that somewhere near the middle the men are much more nearly of the same height, or rather there are more men having about the same height than there are near the ends of the line. Another arrangement will bring this out better. If we stand in a line all the men from 60 to 6 I.9 inches, and in another parallel line all those between 62 and 63.9, then those between 64 and 65.9 , then between 66 and 67.9 inches in height, etc., it will be found that there are more men in some of these lines than in others. The longest line will be that containing the men of about 65 inches; the two lines formed out of men on each side of this one will contain somewhat fewer men, and the next ones fewer still, and so on. If we looked at our new group of men from above, we should have a figure triangular in outline, the so-called frequency polygon, Figure 3 B. With a larger amount of data of this sort it is possible to construct a curve, the curve of frequency, Figure 3 A. In order to obtain this curve of frequency, it is of course not necessary to actually put the indivicluals in line, but the curve can be drawn on paper from the measurements. We sort out the measurements into classes as in the case given above. The classes are laid off at regular intervals along a base-line by placing points at definite intervals. Perpendiculars are then erected at each point, the height of each being proportional to the frequency with which each class occurs. If now we join the tops of these perpendiculars, the curve of frequency is the result.

"In arranging the individuals it will be found, as has been said, that certain groups contain more individuals. They will form the longest line. This value that occurs with the greatest frequency is called the mode. The position of this modal class in the polygon is one of the points of importance, and the spread of the polygon at its base is another. A polygon with a low mode and a broad range means great variability. The range may, however, be much affected by a 
single individual standing far removed from the rest, so that a polygon containing such an individual might appear to show greater variation than really exists. Therefore we need a measure of variability that shall take into account the

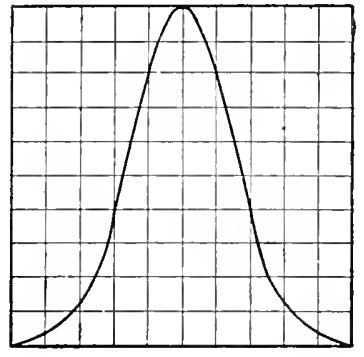

A

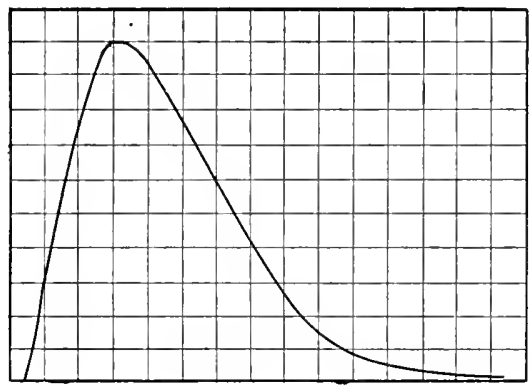

C

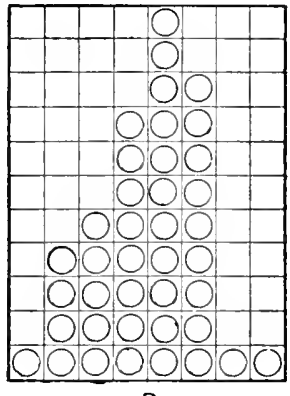

B

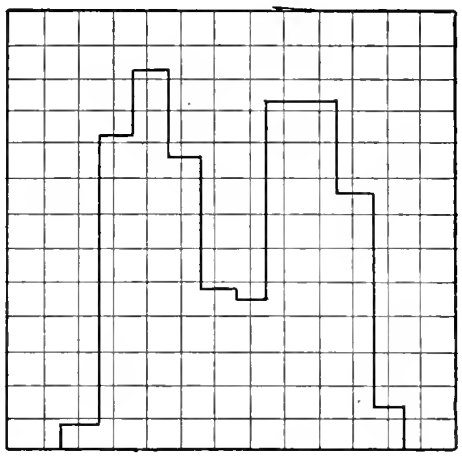

D

FIG. 3. - Curves of frequency, etc. A, normal curve. B, showing the method of arranging individuals in lines containing similar kinds of individuals. $\mathrm{C}$, curve that is skew to the right. D, polygon of frequencies of horns of rhinoceros beetles. (After Davenport.)

departures of all the individuals from the mode. One such measure is the arithmetical average of all the departures from the mean in both directions; and this measure has been widely employed. At present another method is preferred, namely, the square root of the squared departures. This measure is called the standard deviation. The standard 
deviation is of great importance, because it is the index of variability." 1

Of the different kinds of polygons there are two main sorts, the simple and the complex. The former have only a single mode, the latter have more than one mode. Some simple polygons lie symmetrically on each side of the mode, Figure $3 \mathrm{~A}$; others are unsymmetrical or skew, Figure $3 \mathrm{~B}$. The skew polygon generally extends out on one side farther than on the other. It has been suggested that when a polygon is symmetrical the species is not changing, and when skew that the species is evolving in the direction of the longer base. This assumes that the sort of variation measured by these curves is of the kind of which evolution is made up, but this is a question that we must further consider. How far the change indicated by the skew curve may be carried is also another point for further examination.

A complex polygon of variation, Figure $3 \mathrm{D}$, has been sometimes interpreted to mean that two subgroups exist in a species, as is well shown in the case of the rhinoceros beetle described by Bateson. Two kinds of male individuals exist, some with long horns, others with short horns; each with a mode of its own, the two polygons overlapping. Other complex polygons may be due to changes occurring at different times in the life of the individual, as old age, for example.

If, instead of examining the variations of the inclividuals of the race, we study the variations in the different organs of the same individual, we find in many cases that certain organs vary together. Thus the right and the left leg nearly always vary in the same direction, also the first joints of the index and middle fingers, and the stature and the forearm. On the other hand, the length of the clavicle and that of the humerus do not vary together to the same extent; and the breadth and height of the skull even less so.

\footnotetext{
'Davenport, C. B., "The Statistical Study of Biological Problems," Popular Science Monthly, September, Iyoo.
} 
We may also study those cases in which a particular organ is repeated a number of times in the same individual, as are the leaves of a tree. If the leaves of the same tree are examined in respect, for example, to the number of veins that each contains, we find that the number varies, and that the results give a variation polygon exactly like that when different individuals are compared with one another. Let us take the illustration given by Pearson. He counted the veins on each side of the midrib of the leaves of the beech. If a number of leaves be collected from one tree, and the same number from another, and if all those having fifteen veins are put in one vertical column, and all those with sixteen in another, as shown in the following table, it will be found that

\begin{tabular}{|c|c|c|c|c|c|c|c|c|c|c|c|c|c|c|}
\hline No. of Veins & . & IO & I I & I 2 & I 3 & 14 & I 5 & I 6 & I 7 & 18 & I9 & 20 & 21 & 22 \\
\hline First Tree. & . & 一 & 一 & - & - & 一 & I & 4 & 7 & 9 & 4 & I & - & - \\
\hline Second Tree & . & - & - & - & 3 & 4 & 9 & 8 & 2 & - & - & - & 一 & - \\
\hline
\end{tabular}

each tree has a mode of its own. Thus in the first tree the mode is represented by nine individuals having eighteen veins, and in the second by nine individuals having fifteen veins. So far as this character is concerned we might have interchanged certain of the individual leaves, but we could not have interchanged the two series. They are individual to the two trees. Now in what does this individuality consist? Clearly there are most leaves in one tree with eighteen ribs, and most in the other with fifteen ribs.

If we contrast these results with those obtained by picking at random a large number of leaves from different beech trees, we have no longer types of individuals, but racial characters. Pearson has given the following table to illustrate these points:

Frequency of Different Types of Beech Leaves

\begin{tabular}{l|c|c|c|c|c|c|c|c|c|c|c|c|c}
\hline No. of Veins & I0 & I I & I 2 & I 3 & I 4 & I 5 & I6 & I 7 & I 8 & 19 & 20 & 2 I & 22 \\
\hline Frequency & I & 7 & 34 & I 10 & 3 I 8 & 479 & 595 & 5 I6 & 307 & I 8 I & 36 & I 5 & I \\
\hline
\end{tabular}


Thus the mode for beech trees in general is sixteen; but, as shown in the other table, this mode does not correspond with either of the two individual modes here ascertained. The illustration shows that the racial mode may differ from the individual mode. There are also cases known in which the mode of a group of individuals living in one locality is different from that of another group living in another locality. This difference may be a constant one from year to year, although so slight, that unless actual measurements are made, the difference cannot be detected, because of the overlapping of the individuals from different localities. If evolution took place by slow changes of this sort, it might be possible to detect its action, even when very slow, by means of measurements made on a large number of individuals. At least this has been suggested by those who believe new species may result from changes of this sort.

There is some evidence showing that by selecting particular individuals of a series, and breeding from them, the mode may be changed in the direction of selection. Thus it has been stated by Davenport that the descendants of twelveand thirteen-rayed daisies give a polygon with a skewness of +1.92 ; while the descendants of twenty-one-rayed plants give a polygon with a skewness of -.13 .

Pearson has described very concisely the possibilities involved in the selective action of the enviromment. He states that if we examine the frequency distribution of a set of organisms that have just become mature, and later make a similar examination on the same number of individuals (but not the same individuals) during the period of reproduction, we shall probably find that a change has taken place which may have been due to selection of some sort. The same thing might be found in the next generation, and, if it did, this would indicate that "selection does not necessarily mean a permanent or a progressive change." The selection in this imaginary case would be purcly periodic and suffice only to 
maintain a given race under given conditions. "Each new adolescent generation is not the product of the entire preceding generation, but only of selected individuals. This is certainly the case for civilized man, in which case twenty-six per cent of the married population produce fifty per cent of the next generation."

Pearson believes that "if a race has been long under the same environment it is probable that only periodic selection is at work, maintaining its stability. Change the environment and a secular change takes place, the deviations from the mode previously destroyed giving the requisite material." "Clearly periods of rapidly changing environment, of great climatological and geological change, are likely to be associated with most marked secular selection. To show that there is little or no change year by year in the types of rabbit and wild poppy in our English fields, or of daphnia in our English ponds, is to put forward no great argument for the inefficiency of natural selection. Take the rabbit to Australia, the wild poppy to the Cape, the daphnia into the laboratory, and change their temperature, their food supply, and the chemical constituents of water and air, and then the existence of no secular selection would indeed be a valid argument against the Darwinian theory of evolution." In regard to the last point, it should be noted that, even if under the changed conditions a change in the mode took place, as Pearson assumes, it does not follow necessarily that selection has had anything to do with it, but the environment may have directly changed the forms. Furthermore, and this is the essential point, even if selection does act to the extent of changing the mode, we should not be justified in concluding that this sort of change could go on increasing as long as the selection lasts. All that might happen would be to keep the species up to the highest point to which fluctuating variation can be held. This need not lead to the formation of new species, or direct the course of evolution. 
Pearson points out further that, even if we suppose that a secular change is produced in a new environment, we cannot explain how species may break up into two or more races that are relatively infertile. Suppose two groups of individuals, subjected to different environments, become isolated geographically. Two local races will be produced. "Isolation may account for the origin of local races, but never for the origin of species unless it is accompanied by a differential fertility." In other words, Pearson thinks that, unless the reproductive organs are correlated with other organs, in such a way that as these organs change the interracial fertility of the germ-cells is altered, so that in the two changed groups the individuals are no longer interfertile, new species cannot be accounted for, since their mutual infertility is one of their most characteristic features. "Without a barrier to intercrossing during differentiation the origin of species seems inexplicable."

We need not discuss the various suggestions that have been made to explain this difficulty, none of which, as Pearson points out, have been satisfactory. He himself belieres that a process of segregation of like individuals must occur, during the incipient stages at least, in the formation of species. Afterwards a correlation may exist between the new organs and the germ-cells, of such a sort that a relative or an absolute sterility between the incipient species is attained. After this condition has been reached the two new species may freely intermix without a return to the primitive type, since they are no longer fertile inter sc. It seems to me, also, that this would be an essential requisite if we assume that species are slowly formed out of races from individual differences, as Pearson supposes to be the case. There are, however, other possibilities that Pearson does not take into account, namely, that from the very beginning the change may be so great that the new form is not fertile with the original one; and there is also another possibility as well, that, although the 
new and the old forms are fertile, the hybrids may be like one or the other parent, as in several cases to be given later. Not that I mean to say that in either of these two ways can we really offer a solution of the question of infertility, for, from the evidence that we possess, it appears improbable that the infertility of species inter se has been the outcome of either of these causes.

In support of his main thesis Pearson gives certain data in respect to preferential mating in the human race. By this is meant that selection of certain types of individuals is more likely to take place, and also that the fertility of certain types of individuals is greater than that of other types. The calculations are based on stature, color of hair, and of eyes. The results appear to show in all cases examined that there is a slight tendency to form new races as the result of the more frequent selection of certain kinds of individuals. But even if this is the case, what more do the results show than that local races may be formed, - races having a certain mode for height, for color of eyes or of hair? That changes of this kind can be brought about we knew already without any elaborate measurements, yet we should not conclude from this that new species will be formed by a continuation of the process.

Pearson writes: "As to the problem of evolution itself we are learning to see it under a new light. Natural selection, combined with sexual selection [by which Pearson means segregation of certain types through individual selection] and heredity, is actually at work changing types. We have quantitative evidence of its effects in many directions." Yes! but no evidence that selection of this sort can do anything more than keep up the type to the upper limit attained in each generation by fluctuating variations. Pearson adds, "Variations do not occur accidentally, or in isolated instances; autogamic and assortive mating are realities, and the problem of the near future is not whether Darwinism is a reality, but 
what is quantitively the rate at which it is working and has worked." This statement expresses no more than Pearson's conviction that the process of evolution has taken place by means of selection. He ignores other possibilities, which if established may put the whole question in a very different light.

\section{Heredity and Continuous Variation}

It has been to a certain extent assumed in the preceding pages that both parents are alike, or, if different, that they have an equal influence on the offspring. This may be true in many cases for certain characteristics. Thus a son from a tall father and a short mother may be intermediate in height, or if the father is white and the mother black, the children are mulattoes. But other characters rarely or never blend. In such cases the offspring is more like one or the other parent, in which case the inheritance is said to be exclusive. Thus if one parent has blue eyes and the other black, some of the children may have black eyes and others blue. There are also cases of particular inheritance where there may be patches of color, some like the color of one parent, some like that of the other parent. The latter two kinds of inheritance will be more especially considered in the subsequent part of this chapter; for the present we are here chiefly concerned with blended characters.

How much in such cases does each parent contribute to the offspring? This has been expressed by Galton in his law of ancestral heredity. This law takes into account not only the two parents, but also the four grandparents, and the eight great-grandparents, etc. There will be I024 in the tenth generation. These ro24 individuals may be taken as a fair sample of the general population, provided there has not been much interbreeding. Are we then to look upon the individual as the fused or blended product of the population a few generations back? If this were true, should we not expect 
to find all the inclividuals of a community very much alike, except for the fluctuating variations close around the mode?

As a result of his studies on the stature of man, and on the coat color of the Basset hounds, Galton has shown that the inheritance from the parents can be represented by the fraction $\frac{1}{2}$; that is one-half of the peculiarities of the individual comes from the two parents. The four grandparents together count for $\frac{1}{4}$ of the total inheritance, the great-grandparents $\frac{1}{8}$, and so on, giving the series $\frac{1}{2}, \frac{1}{4}, \frac{1}{8}$. Pearson, taking certain other points into consideration, believes the following series more fully represents the inheritance from the ancestors, .3, . I 5, .075, .0375, etc. He concludes that, "if Darwinism be the true view of evolution, i.c. if we are to describe evolution by natural selection combined with heredity, then the law which gives us definitely and concisely the type of the offspring in terms of the ancestral peculiarities is at once the foundation stone of biology and the basis upon which heredity becomes an exact branch of science."

The preceding statements give some idea of what would occur in a community in which no selection was taking place. The results will be quite different, although the same general law of inheritance will hold, if selection takes place in each generation. If, for instance, selection takes place, the offspring after four generations will have .93 of the selected character, and without further selection will not regress, but breed true to this type." "After six generations of selection the offspring will, selection being suspended, breed true to under two per cent divergence from the previously selected type."

If, however, we do not assume that the ancestors were mediocre, it is found that after six generations of selection the offspring will breed true to the selected type within one per cent of its value. Thus, if selection were to act on a race

1 In this statement the earlier ancestors are assumed to be identical with the general type of the population. 
of men having a mode of 5 feet 9 inches, and the 6-foot men were selected in each generation, then in six generations this type would be permanently established, and this change could be effected in two hundred years. ${ }^{1}$

Thus we have exact data as to what will happen on the average when blended, fluctuating variations are selected. Important as such data must always be to give us accurate information as to what will occur if things are left to "chance" variations, yet if it should prove true that evolution has not been the outcome of chance, then the method is entirely useless to determine how evolution has occurred.

More important than a knowledge of what, according to the theory of chances, fluctuating variations will do, will be information that would tell us what changes will take place in each individual. In this field we may hope to obtain clata no less quantitative than those of chance variations, but of a different kind. A study of some of the results of discontinuous variation will show my meaning more clearly.

\section{Discontinuous Variation}

Galton, in his book on "Natural Inheritance," points out that "the theory of natural selection might dispense with a restriction for which it is difficult to see either the need or the justification, namely, that the course of evolution always proceeds by steps that are severally minute and that become effective only through accumulation." An apparent reason, it is suggested, for this common belief "is founded on the fact that whenever search is made for intermediate forms between widely divergent varieties, whether they are of plants or of animals, of weapons or utensils, of customs, religion, or language, or of any other product of evolution, a long and orderly series can usually be made out, each member of which differs in an almost imperceptible degree from the adjacent

1 Quoted from Pearson's "Grammar of Science." 
specimens. But it does not at all follow because these intermediate forms have been found to exist, that they were the very stages that were passed through in the course of evolution. Counter evidence exists in abundance, not only of the appearance of considerable sports, but of their remarkable stability in hereditary transmission." Comparing such an apparently continuous series with machines, Galton concludes, "If, however, all the variations of any machine that had ever been invented were selected and arranged in a museum, each would differ so little from its neighbors as to suggest the fallacious inference that the successive inventions of that machine had progressed by means of a very large number of hardly discernible steps."

Bateson, also, in his "Materials for the Study of Variation," speaks of the two possible ways in which variations may arise. He points out that it has been tacitly assumed that the transitions have been continuous, and that this assumption has introduced many gratuitous difficulties. Chicf of these is the difficulty that in their initial and imperfect stages many variations would be useless. "Of the objections that have been brought against the Theory of Natural Selection, this is by far the most serious." He continues: "The same objection may be expressed in a form which is more correct and comprehensive. We have seen that the differences between species on the whole are Specific, and are differences of kind forming a discontinuous Series, while the diversities of environment to which they are subject are, on the whole, differences of degree, and form a continuous Series; it is, therefore, hard to see how the environmental differences can thus be made in any sense the directing cause of Specific differences, which by the Theory of Natural Selection they should be. This objection of course includes that of the utility of minimal Variations."

"Now the strength of this objection lies wholly in the supposed continuity of the process of Variation. We see all 
organized nature arranged in a discontinuous series of groups differing from each other by differences which are Specific; on the other hand, we see the diverse environments to which these forms are subject passing insensibly into each other. We must admit, then, that if the steps by which the diverse forms of life have varied from each other have been insensible, - if, in fact, the forms ever made up a continuous series, - these forms cannot have been broken into a discontinuous series of groups by a continuous environment, whether acting directly as Lamarck would have, or as selective agent as Dar. win would have. This supposition has been generally made and admitted, but in the absence of evidence as to Variation it is nevertheless a gratuitous assumption, and, as a matter of fact, when the evidence as to Variation is studied, it will be found to be in a great measure unfounded."

There is a fair number of cases on record in which discontinuous variations have been seen to take place. Darwin himself has given a number of excellent examples, and Bateson, in the volume referred to above, has brought together a large and valuable collection of facts of this kind.

Some of the most remarkable of these instances have been already referred to and need only be mentioned here. The black-shouldered peacock, the ancon ram, the turnspit dog, the merino sheep, tailless and hornless animals, are all cases in point. In several of these it has been discovered that the young inherit the peculiarities of their parents if the new variations are bred together; and what is more striking, if the new variation is crossed with the parent form, the young are like one or the other parent, and not intermediate in character. This latter point raises a question of fundamental importance in connection with the origin of species.

Darwin states that he knows of no cases in which, whon

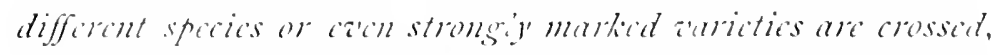
the hybrits are lite one form or the other. They show, he be lieves, always a blending of the peculiarities of the two parents. 
He then makes the following significant statement: "All the characters above enumerated which are transmitted in a perfect state to some of the offspring and not to others such as distinct colors, nakedness of skin, smoothness of leaves, absence of horns or tail, additional toes, pelorism, dwarfed structure, etc., have all been known to appear suddenly in individual animals or plants. From this fact, and from the several slight, aggregated differences which distinguish domestic races and species from each other, not being liable to this peculiar form of transmission, we may conclude that it is in some way connected with the sudden appearance of the characters in question."

Darwin has, incidentally, raised here a question of the most far-reaching import. If it should prove true, as he believes, that inheritance of this kind of discontinuous variation is also discontinuous, and that we do not get the same result when distinct species are intercrossed, or even when well-marked domestic races are interbred, then he has, indeed, placed a great obstacle in the path of those who have tried to show that new species have arisen through discontinuous variation of this sort.

If wild species, when crossed, give almost invariably intermediate forms, then it may appear that we are going against the only evidence that we can hope to obtain if we claim that discontinuous variation, of the kind that sports are made of, has supplied the material for evolution. If, furthermore, when distinct races of domesticated animals are crossed, we do not get discontinuous inheritance, it might, perhaps, with justness be claimed that this instance is paralleled by what takes place when wild species are crossed. And if domesticated forms have been largely the result of the selection of fluctuating variations, as Darwin believes, then a strong case is apparently made out in favor of Darwin's view that continuous variation has given the material for the process of evolution in nature. Whether selection or some other factor 
has directed the formation of the new species would not, of course, be shown, nor would it make any difference in the present connection.

Before we attempt to reach a conclusion on this point let us analyze the facts somewhat more closely.

In the first place, a number of these cases of discontinuous variation are of the nature of abnormalities. The appearance of extra fingers or toes in man and other mammals is an example of this sort. This abnormality is, if inherited at all, inherited completely ; that is, if present the extra digit is perfect, and never appears in an intermediate condition, eren when one of the parents was without it. The most obvious interpretation of this fact is that when the material out of which the fingers are to develop is divided up, or separated into its component parts, one more part than usual is laid down. Similarly, when a flower belonging to the triradiate type gives rise to a quadriradiate form, - as sometimes occurs, - the new variation seems to depend simply on the material being subdivided once more than usual; perhaps because a little more of it is present, or because it has a somewhat different shape. My reasons for making a surmise of this sort are based on certain experimental facts in connection with the regeneration of animals. It has been shown in several cases that it is possible to produce more than the normal number of parts by simply dividing the material so that each part becomes more or less a new whole, and the total number of parts into which the material becomes subdivided is increased. It seems not improbable that phenomena of this sort have occurred in the course of evolution, although it is, of course, possible that those characters that define species do not belong to this class of variation. To take an example. There are nine neck-vertebre in some birds, but in the swan the number is twenty-fire. We cannot suppose that the ancestor of the swan gradually added enough materially to make up one new vertebra and then another, 
but at least one new whole vertebra was added at a time; and we know several cases in which the number of vertebræ in the neck has suddenly been increased by the addition of one more than normal, and the new vertebra is perfectly formed from the first.

In cases of this sort we can easily understand that the inheritance must be either of one kind or the other, since intermediate conditions are impossible, when it comes to the question of one or not one; but if one individual had one and another six vertebra, then it would be theoretically possible for the hybrid to have three.

This brings us to a question that should have been spoken of before in regard to the inheritance of discontinuous variation. It sometimes occurs that a variation, which appears in other respects to be discontinuous, is inherited in a blended form. Thus the two kinds of variation may not always be so sharply separated as one might be led to believe. There may be two different kinds of discontinuous variation in respect to inheritance, or there may be variations that are only to a greater or a less extent inherited discontinuously; and it seems not improbable that both kinds occur.

This diversion may not appear to have brought us any nearer to the solution of the difficulty that Darwin's statement has emphasized, except in so far as it may show that the lines are not so sharply drawn as may have seemed to be the case. The solution of the difficulty is, I believe, as follows :-

The discontinuity referred to by Darwin velates to cases in which only a single stcp (or mutation) has been taken, and it is a question of inheritance of one or not one. If, however, sixsuccessive steps shonld be taken in the same direction, then when such a form is crossed with the original form, the hybrid may' inherit only three of the steps and stand cxactly' midri'cy" betwecn the parent forms; or it may inherit four, or five, or three, or two steps and stand correspondingly' nearer to the one 
or to the other parent. Thus while it may not be possible to hatie a single step (hence one-sided inheritance), yet when more than one step has becn taken the inheritance may be dirided. There is coery evidence that most of the Linnean (wild) species that Darwin refers to ha'e diacrged from the parent form, and from each other, by a number of successia'c steps; hence on crossing, the hybrid often stands somewhere betie'ten the two parent forms. On this basis not only can aie meet Darwin's objection, but the point of vica gives an intcresting insight into the problem of inhcritance and the formation of spccics.

The whole question of inheritance has assumed a new aspect; first on account of the work of De Vries in regard to the appearance of discontinuous variation in plants; and secondly, on account of the remarkable discoveries of Gregor Mendel as to the laws of inheritance of discontinuous variations. Mendel's work, although done in I865, was long neglected, and its importance has only been appreciated in the last few years. We shall take up Mendel's work first, and then that of De Vries.

\section{MeNDEL'S LAW ${ }^{1}$}

The importance of Mendel's results and their wide application is apparent from the results in recent years of De Vries, Correns, Tschermak, Bateson, Castle, and others. Mendel carried out his experiments on the pea, Pisum sativnm. Twenty-two varieties were used, which had been proven by experiment to be pure breeds. When crossed they gave perfectly fertile offspring. Whether they all have the value of varieties of a single species, or are different subspecies, of even independent species, is of little consequence so far as

" Rateson, in his look on "Mendel's l'rinciples of Heredity", has given an admirable presentation of Mendel's results. I have relied largely on this in my account. 
Mendel's experiments are concerned. The flower of the pea is especially suitable for experiments of this kind. It cannot be accidentally fertilized by foreign pollen, because the reproductive organs are inclosed in the keel of the flower, and, as a rule, the anthers burst and cover the stigma of the same flower with its own pollen before the flower opens. In order to cross-fertilize the plants it is necessary to open the young buds before the anthers are mature and carefully remove all the anthers. Foreign pollen may be then, or later, introduced.

The principle involved in Mendel's law may be first stated in a theoretical case, from which a certain complication that appears in the actual results may be removed.

If $A$ represent a variety having a certain character, and $B$ another variety in which the same character is different, let us say in color, and if these two individuals, one of each kind, are crossed, the hybrid may be represented by $H$. If a number of these hybrids are bred together, their descendants will be of three kinds; some will be like the grandparent, $A$, in regard to the special character that we are following, some will be like the other grandparent, $B$, and others will be like the hybrid parent, $H$. Moreover, there will be twice as many with the character $H$, as with $A$, or with $B$.

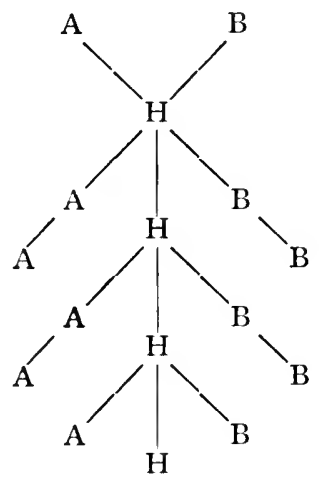

If now we proceed to let these $A$ 's breed together, it will be found that their descendants are all $A$, forever. If the 
$B$ "s are bred together they produce only $B$ 's. But when the $H$ 's are bred together they give rise to $H$ 's, $A$ 's, and $B$ 's, as shown in the accompanying diagram. In each generation, the $A$ 's will also breed true, the $B$ 's true, but the $H$ 's will give rise to the three kinds again, and always in the same proportion.

Thus it is seen that the hybrid individuals continue to give off the pure original forms, in regard to the special character under consideration. The numerical relation between the numbers is also a striking fact. Its explanation is, however, quite simple, and will be given later.

In the actual experiment the results appear somewhat more complicated because the hybrid cannot be distinguished from one of the original parents, but the results really conform exactly to the imaginary case given above. The accompanying diagram will make clearer the account that follows.

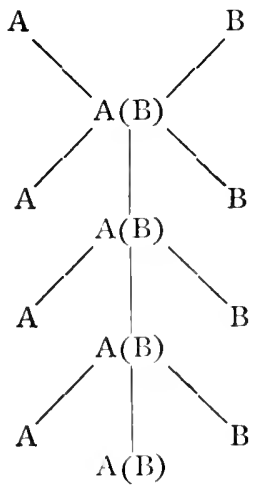

The hybrid, $A(B)$, produced by crossing $A$ and $B$ is like $A$ so far as the special character that we will consicler is concerned. In reality the character that $A$ stands for is only dominant, that is, it has been inherited discontinuously, while the other character, represented by $B$, is latent, or recessive as Mendel calls it. Therefore, in the table, it is included in parentheses. If the hybrids, represented by this form $A(B)$, are bred 
together, there are produced two kinds of individuals, $A$ 's and $B$ 's, of which there are three times as many $A$ 's as $B$ 's. It has been found, however, that some of these $A$ 's are pure forms, as indicated by the $A$ on the left in our table, while the others, as shown by their subsequent history, are hybricls, $A(B)$. There are also twice as many of these $A(B)$ 's as of the pure $A$ 's (or of the $B$ 's). Thus the results are really the same as in our imaginary case, only obscured by the fact that the $A$ 's and the $A(B)$ 's are exactly alike to us in respect to the character chosen. We see also why there appear to be three times as many $A$ 's as $B$ 's. In reality the results are I $A, 2 A(B)$, I $B$.

In subsequent generations the results are the same as in this one, the $A$ 's giving rise only to $A$, the $B$ 's to $B$, and the $A(B)$ 's continuing to split up into the three forms, as shown in our diagram. Mendel found the same law to hold for all the characters he examined, including such different ones as the form of the seed, color of seed-albumen, coloring of seed-coat, form of the ripe pods, position of flowers, and length of stem.

Mendel also carried out a series of experiments in which several clifferentiating characters are associated. In the first experiment the parental plants (varieties) differed in the form of the seed and in the color of the albumen. The two characters of the seed plant are designated by the capital letters $A$ and $B$; and of the pollen plant by small $a$ and $b$. The hybrids will be, of course, combinations of these, although only certain characters may dominate. Thus in the experiments, the parents are $A B$ (seed plant) and $a b$ (pollen plant), with the following seed characters :$\underset{A B}{\text { Seed parent }}\left\{\begin{array}{l}\mathrm{A} \text { form round } \\ \mathrm{B} \text { albumen yellow }\end{array} \quad \begin{array}{c}a b\end{array}\right.$

When these two forms were crossed the seeds appeared round and yellow like those of the parent, $A B$, i.e. these two characters dominated in the hybrid. 
The seeds were sown, and in turn yielded plants which when self-fertilized gave four kinds of seeds (which frequently all appeared in the same pod). Thus 556 seeds were produced by 15 plants, having the following characters :-

$A B 3$ I 5 round and yellow $A b$ Io I angular and yellow $a B$ Io8 round and green $a b 32$ angular and green

These figures stand almost in the relation of $9: 3: 3: 1$.

These seeds were sown again in the following year and gave :-

From the round yellow seeds :-

$A B 38$ round and yellow seeds

$A B b 65$ round yellow and green seeds $A a B 60$ round yellow and angular yellow seeds $A a B b$ i 38 round yellow and greer, angular yellow and green seeds

From the angular yellow seeds :$a B 28$ angular yellow seeds $a B b 68$ angular yellow and green seeds

From the round green seeds :-

$A b 35$ round green seeds $A a b 67$ round angular seeds

From the angular green seeds :$a b 30$ angular green seeds

Thus there were 9 different kinds of seeds produced. There had been separated out at this time ${ }_{3} 8$ individuals like the parent seed plant, $A B$, and 30 like the parent pollen plant, $a b$. Since these had come from similar seeds of the preceding generation they may be looked upon as pure at this time. The forms $A b$ and $a B$ are also constant 
forms which do not subsequently vary. The remainder are still mixed or hybrid in character. By successive self-fertilizations it is possible gradually to separate out from these the pure types of which they are compounded.

Without going into further detail it may be stated that the offspring of the parent hybrids, having two pairs of differentiating characters, are represented by the series:-

$$
A B \quad A b a b \quad a b 2 A B b \quad 2 a b b=2 A a b 2 A B a \quad 2 A a b b
$$

This series is really a combination of the two series :-

$$
\begin{aligned}
& A+2 A a+a \\
& B+2 B b+b
\end{aligned}
$$

Mendel even went farther, and used two parent varieties having three differentiating characters, as follows :-

$A B C$ seed parent

$$
\left\{\begin{array}{l}
\text { A form round } \\
B \text { albumen yellow } \\
C \text { seed-coat grey brown }
\end{array}\right.
$$

abc pollen plant

$$
\left\{\begin{array}{l}
\text { a form angular } \\
b \text { albumen green } \\
c \text { seed-coat white }
\end{array}\right.
$$

The results, as may be imagined, were quite complex, but can be expressed by combining these series :-

$$
\begin{aligned}
& A+2 A a+a \\
& B+2 B b+b \\
& C+2 C c+c
\end{aligned}
$$

In regard to the two latter experiments, in which two and three characters respectively were used, it is interesting to point out that the form of the hybrid more nearly approaches "to that one of the parental plants which possesses the greatest number of dominant characters." If, for instance, the seed plant has short stem, terminal white flowers, and simply inflated pods; the pollen plant, on the other hand, a long stem, violet-red flowers distributed along the stem, and constricted pods, - then the hybrid resembles the seed parent only in the form of the pod; in its other characters it agrees 
with the pollen plant. From this we may conclude that, if two varieties differing in a large number of characters are crossed, the hybrid might get some of its dominant characters from one parent, and other dominant characters from the other parent, so that, unless the individual characters themselves were studied, it might appear that the hybrids are intermediate between the two parents, while in reality they are only combinations of the dominant characters of the two forms. But even this is not the whole question.

Mendel points out that, from knowing the characters of the two parent forms (or varieties), one could not prophesy what the hybrid would be like without making the actual trial. Which of the characters of the two parent forms will be the dominant ones, and which recessive, can only be determined by experiment. Moreover, the hybrid characters are something peculiar to the hyb-id itself, and to itself alone, and not simply the combination of the characters of the two forms. Thus in one case a hybrid from a tall and a short variety of pea was even taller than the taller parent variety. Bateson lays much emphasis on this point, believing it to be an important consideration in all questions relating to hybridization and inheritance.

The theoretical interpretation that Mendel has put upon his results is so extremely simple that there can be little doubt that he has hit on the real explanation. The results can be accounted for if we suppose that the hybrid produces egg-cells and pollen-cells, each of which is the bearer of only one of the alternative characters, dominant or recessive as the case may be. If this is the case, and if on an average there are the same number of egg-cells and pollencells, having one or the other of these kinds of characters, then on a random assortment meeting of egg-cells and pollen-cells, Mendel's law would follow. For, 25 per cent of dominant pollen grains would meet with 25 per cent dominant egg-cells; 25 per cent recessive pollen grains would meet with 
25 per cent recessive egg-cells; while the remaining 50 per cent of each kind would meet each other. Or, as Mendel showed by the following scheme:--

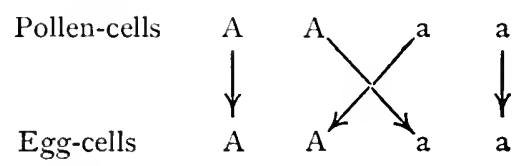

Or more simply by this scheme:-

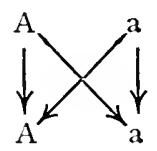

Mendel's results have received confirmation by a number of more recent workers, and while in some cases the results appear to be complicated by other factors, yet there can remain little doubt that Mendel has discovered one of the fundamental laws of heredity.

It has been found that there are some cases in which the sort of inheritance postulated by Mendel's law does not seem to hold, and, in fact, Mendel himself spoke of such cases. $\mathrm{He}$ found that some kinds of hybrids do not break up in later generations into the parent forms. He also points out that in cases of discontinuity the variations in each character must be separately regarded. In most experiments in crossing, forms are chosen which differ from each other in a multitude of characters, some of which are continuous and others discontinuous, some capable of blending with their contraries while others are not. The observer in attempting to discover any regularity is confused by the complications thus introduced. Mendel's law could only appear in such cases by the use of an overwhelming number of examples which are beyond the possibilities of experiment. ${ }^{1}$

Let us now examine the bearing of these discoveries on ${ }^{1}$ This statement is largely taken from Bateson's book. 
the questions of variation which were raised in the prececing pages. It should be pointed out, however, that it would be premature to do more than indicate, in the most general way, the application of these conclusions. The chief value of Mendel's results lies in their relation to the theory of inheritance rather than to that of evolution.

In the first place, Mendel's results indicate that we cannot make any such sharp distinction as Darwin does between the results of inheritance of cliscontinuous and of continuous variations. As Mendel's results show, it is the separate characters that must be considered in each case, and not simply the sum total of characters.

The more general objection that Darwin has made may appear to hold, nevertheless. He thinks that the evolution of animals and plants cannot rest primarily on the appearance of discontinuous variations, because they occur rarely and would be swamped by intercrossing. If Mendel's law applies to such cases, that is, if a cross were made between such a sport and the original form, the hybrid in this case, if self-fertilized, would begin to split up into the two original forms. But, on the other hand, it could very rarely happen that the hybrid did fertilize its own eggs, and, unless this occurred, the hybrid, by crossing with the parent forms in each generation, would soon lose all its characters inherited from its "sport" ancestor. Unless, therefore, other individuals gave rise to sports at the same time, there would be little chance of producing new species in this way. We see then that discontinuity in itself, unless it involved infertility with the parent species, of which there is no evidence, cannot be macle the basis for a theory of evolution, any more than can individual differences, for the swamping effect of intercrossing would in both cases soon obliterate the new form. If, however, a species begins to give rise to a large number of individuals of the same kind through a process of discontinuous variation, then it may happen that a new form may 
establish itself, either because it is adapted to live under conditions somewhat clifferent from the parent form, so that the dangers of intercrossing are lessened, or because the new form may absorb the old one. It is also clear, from what has gone before, that the new form can only cease to be fertile with the parent form, or with its sister forms, after it has undergone such a number of changes that it is no longer able to combine the differences in a new individual. This result will depend both on the kinds of the new characters, as well as the amounts of their difference. This brings us to a consideration of the results of De Vries, who has studied the first steps in the formation of new species in the "mutations" of the evening primrose.

\section{The Mutation Theory of De Vries}

De Vries defines the mutation theory as the eonception that " the characters of the organism are made up of elements ("Einheiten') that are sharply separated from each other. These elements can be combined in groups, and in related species the same combinations of elements recur. Transitional forms like those that are so common in the external features of animals and plants do not exist between the elements themselves, any more than they do between the elements of the chemist."

This principle leads, De Vries says, in the clomain of the descent theory to the conception that species have arisen from each other, not continuously, but by steps. Each new step results from a new combination as compared with the old one, and the new forms are thereby completely and sharply separated from the species from which they have eome. The new species is all at once there; it has arisen from the parent form without visible preparation and without transitional steps.

The mutation theory stands in sharp contrast to the selection theory. The latter uses as its starting-point the com- 
mon form of variability known as individual or fluctuating variation; but according to the mutation theory there are two kinds of variation that are entirely different from each other. "The fluctuating variation can, as I hope to show, not overstep the bounds of the species, even after the most prolonged selection, - much less can this kind of variation lead to the production of new, constant characters." Each peculiarity of the organism has arisen from a preceding one, not through the common form of variation, but through a sudden change that may be quite small but is perfectly definite. This kind of variability that produces new species, De Vries calls mutability; the change itself he calls a mutation. The best-known examples of mutations are those which Darwin called "single variations" or "sports."

De Vries recognizes the following kinds of variation :-

First, the polymorphic forms of the systematists. The ordinary groups which, following Linnæus, we call species, are according to De Vries collective groups, which are the outcome of mutations. Many such Linnzean species include small series of related forms, and sometimes even large numbers of such forms. These are as distinctly and completely separated from each other as are the best species. Generally these small groups are called varieties, or subspecies, - varieties when they are separated by a single striking character, subspecies when they differ in the totality of their characters, in the so-called habitus.

These groups have already been recognized by some investigators as elementary species, and have been given corresponding binary names. Thus there are recognized two hundred elementary species of the form formerly called Drata a'cona.

When brought under cultivation these elementary species are constant in character and transmit their peculiarities truly. They are not local races in the sense that they are the outcome in each seneration of special external conditions. 
Many other Linnæan species are in this respect like Drabu verna, and most varieties, De Vries thinks, are really elementary species.

Second, the polymorphism due to intercrossing is the outcome of different combinations of hereditary qualities. There are here, De Vries says, two important classes of facts to be kept strictly apart, - scientific experiment, and the results of the gardener and of the cultivator. The experimenter chooses for crossing, species as little variable as possible; the gardener and cultivator on the other hand prefer to cross forms of which one at least is variable, because the variations may be transmitted to the hybrid, and in this way a new form be produced.

New elementary characters arise in experiments in crossing only through variability, not through crossing itself.

Third, variability in the ordinary sense, that is, individual variability, includes those differences between the individual organs that follow Quetelet's theory of chance. This kind of variability is characterized by its presence at all times, in all groups of individuals.

De Vries recalls Galton's apt comparison between variability and a polyhedron which can roll from one face to another. When it comes to rest on any particular face, it is in stable equilibrium. Small vibrations or disturbances may make it oscillate, but it returns always to the same face. These oscillations are like the fluctuating variations. A greater disturbance may cause the polyhedron to roll over on to a new face, where it comes to rest again, only showing the ever present fluctuations around its new centre. The new position corresponds to a mutation. It may appear from our familiarity with the great changes that we associate with the idea of discontinuous variability, that a mutation must also involve a considerable change. Such, however, De Vries says, is not the case. In fact, numerous mutations are smaller than the extremes of fluctuating variation. For example, the 
different elementary species of Draba a'crna are less different from each other than the forms of leaves on a tree. The essential differences between the two kinds of variation is that the mutation is constant, while the continuous variation fluctuates back and forth.

The following example is given by De Vries to illustrate the general point of view in regard to varieties and species. The species Oxalis comiculata is a "collective" species that lives in New Zealand. It has been described as having seven wellcharacterized varieties which do not live together or have intermediate forms. If we knew only this group, there would be no question that there are seven good species. But in other countries intermediate forms exist, which exactly bridge over the differences between the seven New Zealand forms. For this reason all the forms have been united in a single species.

Another example is that of the fern, Lomaria procera, from New Zealand, Australia, South Africa, and South America. If the forms from only one country be considered, they appear to be different species; but if all the forms from the different parts of the world be taken into account, they constitute a connected group, and are united into one large species.

It will be seen, therefore, that the limits of a collective species are determined solely by the deficiencies in the genealogical tree of the clementary species. If all the elementary species in one country were destroyed, then the forms living in other countries that had been previously held together because of those which have now been destroyed, would, after the destruction, become true species. In other words: "The Linnaean species are formed by the disappearance of other elementary species, which at first connected all forms. This mode of origin is a purely historical process, and can never become the subject of experimental investigation." Spencer's famous expression, the "survival of the fittest," is incomplete, and should read the "survival of the 
fittest species." It is, therefore, not the study of Linnæan species that has a physiological interest, but it is the study of the elementary species of which the Linnæan species are made up, that furnishes the all-important problem for experimental study.

De Vries gives a critical analysis of a number of cases in which new races have been formed under domestication. He shows very convincingly that, whenever the result has been the outcome of the selection of fluctuating variations, the product that is formed can only be kept to its highest point of development by the most rigid and ever watchful care. If selection ceases for only a few generations, the new form sinks back at once to its original level. Many of our cultivated plants have really arisen, not by selection of this sort, but by mutations; and there are a number of recorded cases where the first and sudden appearance of a new form has been observed. In such cases as these there is no need for selection, for if left to themselves there is no return to the original form. If, however, after a new mutation has appeared in this way, we subject its fluctuating variations to selection, we can keep the new form up to its most extreme limit, but can do nothing more.

Another means, frequently employed, by which new varieties have been formed is by bringing together different elementary species under cultivation. For instance, there are a large number of wild elementary species of apples, and De Vries believes that our different races of apples owe their origin in part to these different wild forms. Crossing, cultivation, and selection have done the rest.

De Vries points out some of the inconsistencies of those who have attempted to discriminate between varieties and species. The only rule that can be adhered to is that a variety differs from a species to which it belongs in only one or in a few characters. Most so-called varieties in nature are really elementary species, which differ from their nearest 
relatives, not in one character only, but in nearly all their characters. There is no ground, De Vries states, for believing them to be varieties. If it is found inconvenient to rank them under the names of the old Linnaean species, it will be better, perhaps, to treat them as subspecies, but De Vries prefers to call them elementary species.

In regard to the distribution of species in nature, it may be generally stated that the larger the geographical domain so much the larger is the number of elementary species. They are found to be heaped up in the centre of their area of distribution, but are more scattered at the periphery.

In any one locality each Linnzan species has as a rule only one or a few elementary species. The larger the area the more numerous the forms. From France alone Jordan had brought together in his garden 50 elementary species of Draba vorna. From England, Italy, and Austria there could be added I 50 more. This polymorphism is, De Vries thinks, a general phenomenon, although the number of forms is seldom so great as in this case.

Amongst animals this great variety of forms is not often met with, yet amongst the mammalia and birds of North America there are many cases of local forms or races, some of which at least are probably mutations. This can only be proven, however, by actually transferring the forms to new localities in order to find out if they retain their original characters, or become changed into another form. It seems not improbable that many of the forms are not the outcome of the external conditions under which the animal now lives, but would perpetuate themselves in a new environment.

From the evidence that his results have given, De Vries believes it is probable that mutation has occurred in all clirections. In the same way that Darwin supposed that individual or fluctuating variations are scattering, so also De Vries believes that the new forms that arise through mutation are scattering. On this point it seems to me that De Vries may 
be too much prejudiced by his results with the evening primrose. If, as he supposes, many forms, generally ranked as varieties, are really elementary species, it seems more probable that the mutation of a form may often be limited to the production of one or of only a very few new forms. The single variations, or sports, point even more strongly in favor of this interpretation. Moreover, the general problem of evolution from a purely theoretical point of view is very much simplified, if we assume that the kinds of mutating forms may often be very limited, and that mutations may often continue to occur in a direct line. On this last point, De Vries argues that the evidence from paleontology cannot be trusted, for all that we can conclude from fossil remains is that certain mutations have dominated, and have been sufficiently abundant to leave a record. In other words, the conditions may have been such that only certain forms could find a foothold.

De Vries asks whether there are for each species periods of mutation when many and great changes take place, and periods when relatively little change occurs. The evidence upon which to form an opinion is scanty, but De Vries is inclined to think that such periods do occur. It is at least certain from our experience that there are long periods when we do not see new forms arising, while at other times, although we know very few of them, epidemics of change may take place. The mutative period which De Vries found in the evening primrose is the best-known example of such a period of active mutation. Equally important for the descent theory is the idea that the same mutation may appear time after time. There is good evidence to show that this really occurs, and in consequence the chances for the perpetuation of such a form are greatly increased. Delbœuf, who advocated this idea of the repeated reappearance of a new form, has also attempted to show that if this occurs the new form may become established without selection of any kind taking place, 
- the time required depending upon the frequency with which the new form appears. This law of Delbouf, De Vries believes, is correct from the point of view of the mutation theory. It explains, in a very simple way, the existence of numerous species-characters that are entirely useless, such, for instance, as exist between the different elementary species of Draba a'ma. "According to the selection theory only useful characters can survive; according to the mutation theory, useless characters also may survive, and even those that may be hurtful to a small degree."

We may now proceed to examine the evidence from which De Vries has been led to the general conclusions given in the preceding pages. De Vries found at Hilversam, near Amsterdam, a locality where a number of plants of the evening primrose, Enothera lamarkiana, grow in large numbers. This plant is an American form that has been imported into Europe. It often escapes from cultivation, as is the case at Hilversam, where for ten years it had been growing wild. Its rapid increase in numbers in the course of a few years may be one of the causes that has led to the appearance of a mutation period. The escaped plants showed fluctuating variations in nearly all of their organs. They also had produced a number of abnormal forms. Some of the plants came to maturity in one year, others in two, or in rare cases in three, years.

A year after the first finding of these plants De Vries observed two well-characterized forms, which he at once recognized as new elementary species. One of these was $O$. bre ristylis, which occurred only as female plants. The other new species was a smooth-leafed form with a more beautiful foliage than $O$. lamarckiana. This is O.lavifoler. It was found that both of these new forms bred true from self-fertilized seeds. At first only a few specimens were found, each form in a particular part of the field, which looks as though each might have come from the seeds of a single plant. 
These two new forms, as well as the common $O$. lamarkiana, were collected, and from these plants there have arisen the three groups or families of elementary species that De Vries has studied. In his garden other new forms also arose from those that had been brought under cultivation. The largest group and the most important one is that from the original O. lamarkiana form. The accompanying table

\section{ENOTHERA LAMARCKIANA}

Elementary Species

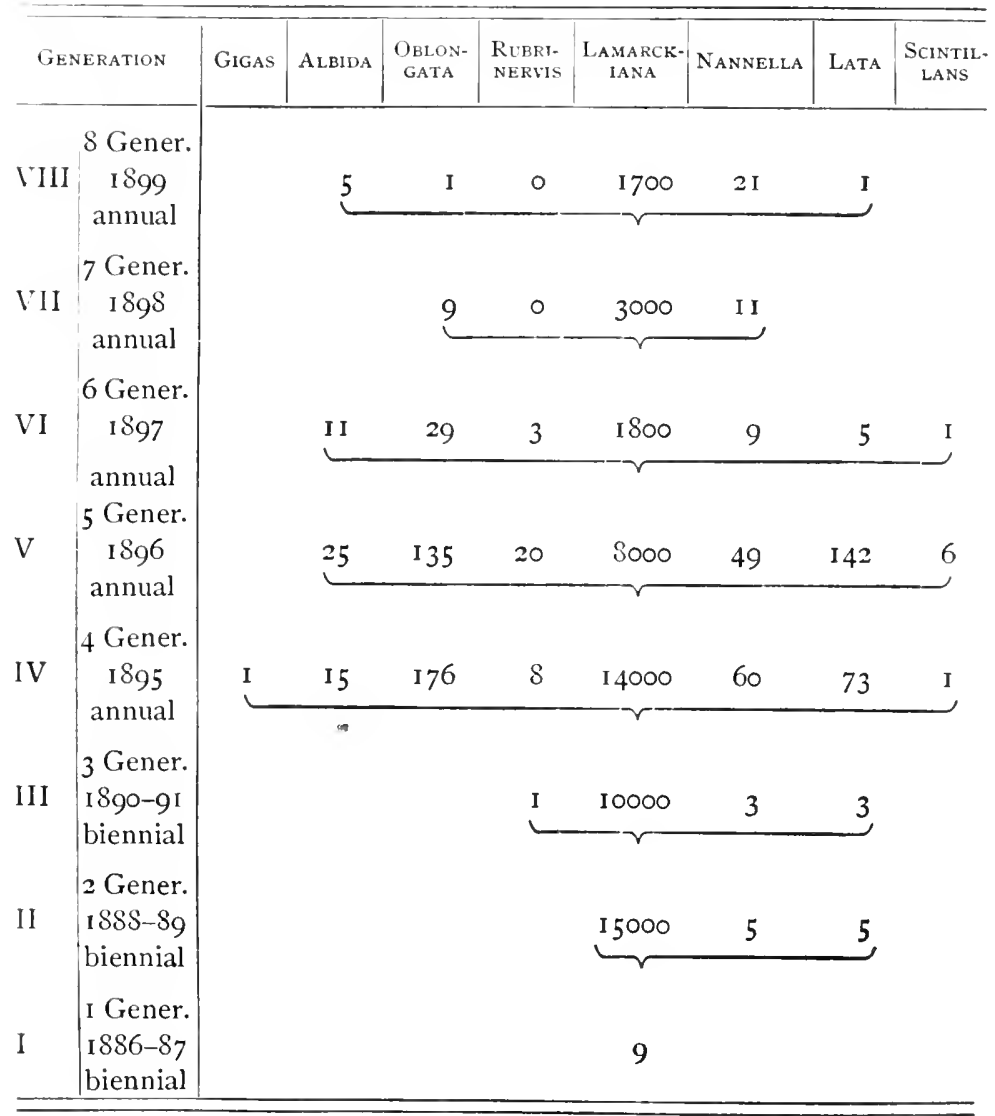


shows the mutations that arose between I 887 and I 899 from these plants. The seeds were selected in each case from self-fertilized plants of the lamarkiana form, so that the new plants appearing in each horizontal line are the descendants in each generation of lamarkiana parents. It will be observed that the species, O. oblongata, appeared again and again in considerable numbers, and the same is true for several of the other forms also. Only the two species, $O$. gigas and O. scintillans, appeared very rarely.

Thus De Vries had, in his seven generations, about fifty thousand plants, and about eight hundred of these were mutations. When the flowers of the new forms were artificially fertilized with pollen from the flowers on the same plant, or of the same kind of plant, they gave rise to forms like themselves, thus showing that they are true elementary species. ${ }^{1}$ It is also a point of some interest to observe that all these forms differed from each other in a large number of particulars.

Only one form, O. scintillans, that appeared eight times, is not constant as are the other species. When self-fertilized its seeds produce always three other forms, $O$. scintillans, O. oblongata, and O. lamarckiana. It differs in this respect from all the other elementary species, which mutate not more than once in ten thousand indivicluals.

From the seeds of one of the new forms, $O$. levifolia, collected in the field, plants were reared, some of which were $O$. lamarckiana and others $O$. larifolia. They were allowed to grow together, and their descendants gave rise to the same forms found in the lamarkiana family, described above, namely, O. lata, clliptica, nannclla, mbrincris, and also two new species, O. spatulata and leptocarpa.

In the lato family, only female flowers are produced, and, therefore, in order to obtain seeds they were fertilized with

1 O. lata is always female, and cannot, therefore, be self-fertilized. When crossed with $O$. lamarckiana there is produced tifteen to twenty per cent of pure lata individuals. 
pollen from other species. Here also appeared some of the new species, already mentioned, namely, albida, nannella, lata, oblongata, nubrincris, and also two new species, clliptica and subovata.

De Vries also watched the field from which the original forms were obtained, and found there many of the new species that appeared under cultivation. These were found, however, only as weak young plants that rarely flowered. Five of the new forms were seen either in the Hilversam field, or else raised from seeds that had been collected there. These facts show that the new species are not due to cultivation, and that they arise year after year from the seeds of the parent form, O. lamarckiana.

\section{Conclusions}

From the evidence given in the preceding pages it appears that the line between fluctuating variations and mutations may be sharply drawn. If we assume that mutations have furnished the material for the process of evolution, the whole problem appears in a different light from that in which it was placed by Darwin when he assumed that the fluctuating variations are the kind which give the material for evolution.

From the point of view of the mutation theory, species are no longer looked upon as having been slowly built up through the selection of individual variations, but the elementary species, at least, appear at a single advance, and fully formed. This need not necessarily mean that great changes have suddenly taken place, and in this respect the mutation theory is in accord with Darwin's view that extreme forms that rarely appear, "sports," have not furnished the material for the process of evolution.

As De Vries has pointed out, each mutation may be different from the parent form in only a slight degree for each point, although all the points may be different. The most unique 
feature of these mutations is the constancy with which the new form is inherited. It is this fact, not previously fully appreciated, that De Vries's work has brought prominently into the foreground. There is another point of great interest in this connection. Many of the groups that Darwin recognized as varieties correspond to the elementary species of De Vries. These varieties, Darwin thought, are the first stages in the formations of species, and, in fact, cannot be separated from species in most eases. The main difference between the selection theory and the mutation theory is that the one supposes these varieties to arise through selection of individual variations, the other supposes that they have arisen spontaneously and at once from the original form. The derelopment of these varieties into new species is again supposed, on the Darwinian theory, to be the result of further selection, on the mutation theory, the result of the appearance of new mutations.

In consequence of this difference in the two theories, it will not be diffictilt to show that the mutation theory escapes some of the gravest difficulties that the Darwinian theory has encountered. Some of the advantages of the mutation theory may be briefly mentioned here.

I. Since the mutations appear fully formed from the beginning, there is no difficulty in accounting for the incipient stages in the development of an organ, and since the organ may persist, even when it has no value to the race, it may become further developed by later mutations and may come to have finally an important relation to the life of the individual.

2. The new mutations may appear in large numbers, and of the different kinds those will persist that can get a foothold. On account of the large number of times that the same mutations appear, the danger of becoming swamped through crossing with the original form will be lessened in proportion to the number of new individuals that arise.

3. If the time of reaching maturity in the new form is different from that in the parent forms, then the new species 
will be kept from crossing with the parent form, and since this new character will be present from the beginning, the new form will have much better chances of surviving than if a difference in time of reaching maturity had to be gradually acquired.

4. The new species that appear may be in some cases already adapted to liye, in a different environment from that occupied by the parent form; and if so, it will be isolated from the beginning, which will be an advantage in avoiding the bad effects of intercrossing.

5. It is well known that the differences between related species consists largely in clifferences of unimportant organs, and this is in harmony with the mutation theory, but one of the real difficulties of the selection theory.

6. Useless or even slightly injurious characters may appear as mutations, and if they do not seriously affect the perpetuation of the race, they may persist.

In Chapters $\mathrm{X}$ and $\mathrm{XI}$, an attempt will be made to point out in detail the advantages which the mutation theory has over the Darwinian theory. 


\section{CHAPTER IX}

EVOLUTION AS THE RESULT OF EXTERNAL AND INTERNAL FACTORS

WE come now to a consideration of other theories that have been advanced to account for the evolution of new forms; and in so far as these new forms are adapted to their enviromment, the theories will bear directly on the question of the origin of adaptive variations. One school of transformationists has made the external world and the changes taking place in it the source of new variations. Another school believes that the changes arise within the organism itself. We may examine these two points of view in turn.

\section{The Effect of External Influences}

We have already seen that Lamarck held as a part of his doctrine of transformation that the changes in the external workd, the environment, bring about, directly, changes in the organism, and he believed that all plants and many of the fower animals have evolved as the result of a reaction of this sort. This idea did not originate with Lamarck, however, since before him Buffon had advanced the same hypothesis, and there cannot be much doubt that Lamarck borrowed from his patron, Buffon, this part of his theory of evolution.

This idea of the influence of the external world as a factor inducing changes in the organism has come, however, to be associated especially with the name of Geoffroy Saint-Hilaire, whose period of activity, athough overlapping, came after 


\section{External and Internal Factors in Evolution $3 \mathrm{OI}$}

that of Lamarck. The central idea of Geoffroy's view was that species of animals and plants undergo change as the environment changes; and it is important to note, in passing, that he did not suppose that these changes were always for the benefit of the individual, i.t. they were not always adaptive. If they were not, the forms became extinct. So long as the conditions remain constant, the species remains constant; and he found an answer in this to Cuvier's argument, in respect to the similarity between the animals living at present in Egypt and those discovered embalmed along with mummies at least two thousand years old. Geoffroy SaintHilaire said, that since the climatic conditions of Egypt had remained exactly the same during all these years, the animals of Egypt would also have remained unchanged.

Geoffroy's views were largely influenced by his studies in systematic zoology and by his conception of a unity of plan running through the entire animal kingdom. His study of embryology and paleontology had led him to believe that present forms have descended from other organisms living in the past, and in this connection his discovery of teeth in the jaws of the embryo of the baleen whale and also his discovery of the embryonic dental ridges in the upper and in the lower jaws of birds, were used with effect in supporting the theory of change or evolution. Lastly, his remarkable work in the study of abnormal forms prepared the way for his conception of sudden and great changes, which he believed organisms capable of undergoing. He went so far in fact, in one instance, as to suppose that it was not impossible that a bird might have issued fully equipped from the egg of a crocodile. Such an extreme statement, which seems to us nowadays only laughable, need not prejudice us against the more moderate parts of his speculation.

His study of the fossil gavials found near Caen led him to believe that they are quite distinct from living crocodiles. He asked whether these old forms may not represent a link 
in the chain that connects, without interruption, the older inhabitants of the earth with animals living at the present time. Without positively affirming that this is the case, he did not hesitate to state that a transformation of this sort seemed possible to him. He said: "I think that the process of respiration constitutes an acquirement so important in the 'disposition' of the forms of animals, that it is not at all necessary to suppose that the surrounding respiratory gases become modified quickly and in large amount in order that the animal may become slowly modified. The prolonged action of time would ordinarily suffice, but if combined with a cataclysm, the result would be so much the better."

He supposed that in the course of time respiration becomes difficult and finally impossible as far as certain systems of organs are concerned. The necessity then arises and creates another arrangement, perfecting or altering the existing structures. Modifications, fortunate or fatal, are created which through propagation are continued, and which, if fortunate, influence all the rest of the organization. But if the modifications are injurious to the animals in which they have appeared, the animals cease to exist, and are replaced by others having a different form, and one suited to the new circumstances.

The comparison between the stages of development of the individual and the evolution of the species was strongly impressed on the mind of Geoffroy. He says: "We see, each year, the spectacle of the transformation in organization from one class into another. A batrachian is at first a fish under the name of a tadpole, then a reptile (amphibian) under that of a frog." "The development, or the result of the transformation, is brought about by the combined action of light and of oxygen; and the change in the body of the animal takes place by the production of new blood-vessels, whose development follows the law of the balancing of organs, in the sense, that if the circulating fluids precipitate themselves into new channels there remains less in the old 
vessels." By preventing tadpoles from leaving the water, Geoffroy claims that it has been shown that they can be prevented from changing into frogs. The main point that Geoffroy attempts to establish is no doubt fairly clear, but the way in which he supposes the change to be effected is not so clear, and his ideas as to the way in which new change may be perpetuated in the next generation are, from our more modern point of view, extremely hazy. It is perhaps not altogether fair to judge his view from the standpoint of the origin of adaptive structures, but rather as an attempt to explain the causes that have brought about the evolution of the organic world.

During the remainder of the nineteenth century there accumulated a large number of facts in relation to the action of the external conditions in bringing about changes in animals and plants. Much of this evidence is of importance in dealing with the question of the origin of organic adaptation.

The first class of facts in this connection is that of geographical variation in animals and plants. It will be impossible here to do more than select some of the most important cases. De Varigny, in his book on "Experimental Evolution," has brought together a large number of facts of this kind, and from his account the following illustrations have been selected. He says: "When the small brown honey-bee from High Burgundy is transported into Bressealthough not very distant - it soon becomes larger and assumes a yellow color; this happens even in the second generation." It is also pointed out that the roots of the beet, carrot, and radish are colorless in their wild natural state, but when brought under cultivation they become red, yellow, etc. Vilmorin has noted that the red, yellow, and violet colors of carrots appear only some time after the wild forms have been brought under cultivation. Moquin-Tandon has seen "gentians which are blue in valleys become white 
on mountains." Other cases also are on record in which the colors of a plant are dependent on external conditions.

The sizes of plants and animals are also often directly traceable to certain external conditions; the change is generally connected with the amount of food obtainable. "Generally speaking," De Varigny says, "insular animals are smaller than their continental congeners. In the Canary Islands the oxen of one of the smallest islands are smaller than those on the others, although all belong to the same breed, and the horses are also smaller, and the indigenous inhabitants are in the same case, although belonging to a tall race. It would seem that in Malta elephants were very small, - fossil elephants, of course, - and that during the Roman period the island was noted for a dwarf breed of dogs, which was named after its birthplace, according to Strabo. In Corsica, also, horses and oxen are very small, and Corins corsicamus, the indigenous deer, is quite reduced in dimensions; . . . and lastly, the small climensions of the Falkland horses imported from Spain in I764-are familiar to all. The dwarf rabbits of Porto Santo described by Darwin may also be cited as a case in point."

These facts, interesting as they are, will, no cloubt, have to be more carefully examined before the evidence can have great value, for it is not clear what factor or factors have produced the decrease in size of these animals.

The following cases show more clearly the immediate effect of the environment: "Many animals, when transferred to warm climates, lose their wool, or their hairy covering is much reduced. In some parts of the warmer regions of the earth, sheep have no wool, but merely hairs like those of dogs. Similarly, as Roulin notices, poultry have, in Columbia, lost their feathers, and while the young are at first covered with a black and delicate down, they lose it in great part as they grow, and the adult fowls nearly realize Plato's realistic description of man - a biped without feathers. 
Conversely, many animals when transferred from warm to cold climates acquire a thicker covering; dogs and horses, for instance, becoming covered with wool."

A number of kinds of snails that were supposed to belong to different species have been found, on further examination, to be only varieties due to the environment. "Locard has discovered through experiments that L. turgida and clophila are mere varieties - due to environment - of the common Lymnea stagnalis." He says, "These are not new species, but merely common aspects of a common type, which is capable of modification and of adaptation according to the nature of the media in which it has to live." It has also been shown by Bateson that similar changes occur in Cardium edulc, and other lamellibranchs are known to vary according to the nature of the water in which they live.

In regard to plants, the influence of the environment has long been known to produce an effect on the form, color, etc., of the individuals. "The common dandelion (Taraxacum donslconis) has in dry soil leaves which are much more irregular and incised, while they are hardly dentate in marshy stations, where it is called Taraxacum palustre.

"Individuals growing near the seashore differ markedly from those growing far inland. Similarly, species such as some Ranunculi, which can live under water as well as in air, exhibit marked differences when considered in their different stations, as is well known to all. These differences may be important enough to induce botanists to believe in the existence of two different species when there is only one."

An interesting case is that of Daplinia rectirostris, a small crustacean living sometimes in fresh water, at other times in water containing salt and also in salt lakes. There are two forms, corresponding to the conditions under which they live, and it is said that the differences are of a kind that suffice to separate species from each other. In another 
crustacean, Branchipus forox, the form differs in a number of points, according to whether it lives in salt or in fresh water. Schmankewitsch says that, had he not found all transitional forms, and observed the transformation in cultures, he would have regarded the two forms as separate species. The oft-quoted case of Artemia furnishes a very striking example of the influence of the environment. Artcmia salina lives in water whose concentration varies between 5 and 12 degrees of saltness. When the amount of salt is increased to 12 degrees, the animal shows certain characteristics like those of Artcmia millanscmil, which may live in water having 24 to 25 degrees of saltness. The form $A$. salina may be further completely changed into that of $A$. milhausenii by increasing the amount of salt to the latter amount.

Among domesticated animals and plants - a few instances of which have been already referred to - we find a large number of cases in which a change in the environment produces definite changes in the organism. Darwin has made a most valuable collection of facts of this kind in his "Animals and Plants under Domestication." He believes that domesticated forms are much more variable than wild ones, and that this is clue, in part, to their being protected from competition, and to their having been removed from their natural conditions and even from their native country. "In conformity with this, all our domesticated productions without exception vary far more than natural species. The hive-bee, which feeds itself, and follows in most respects its natural habits of life, is the least variable of all clomesticated animals. . . Hardly a single plant can be named, which has long been cultivated and propagated by seed, that is not highly variable." "Bud-variation . . . shows us that variability may be quite independent of seminal reproduction, and likewise of reversion to long-lost ancestral characters. No one will maintain that the sudden appearance of 
a moss-rose on a Provence rose is a return to a former state, ... nor can the appearance of nectarines on peach trees be accounted for on the principle of reversion." It is said that bud-variations are also much more frequent on cultivated than on wild plants.

Darwin adds: "These general considerations alone render it probable that variability of every kind is directly or indirectly caused by changed conditions of life. Or to put the case under another point of view, if it were possible to expose all the individuals of a species during many generations to absolutely uniform conditions of life, there would be no variability."

In some cases it has been observed that, in passing from one part of a continent to another, many or all of the forms of the same group and even of different groups change in the same way. Allen's account of the variations in North American birds and mammals furnishes a number of striking examples of this kind of change. He finds that, as a rule, the birds and mammals of North America increase in size from the south northward. This is true, not only for the individuals of the same species, but generally the largest species of each genus are in the north. There are some exceptions, however, in which the increase in size is in the opposite direction. The explanation of this is that the largest individuals are almost invariably found in the region where the group to which the species belongs receives its greatest numerical development. This Allen interprets as the hypothetical "centre of distribution of the species," which is in most cases doubtless also its original centre of dispersal. If the species has arisen in the north, then the northern forms are the largest; but if it arose in the south, the reverse is the case. Thus, most of the species of North America that live north of Mexico are supposed to have had a northern origin, as shown by the circumpolar distribution of some of them and by the relationship of 
others to Old World species; and in these the largest individuals of the species of a genus are northern. Conversely, in the exceptional cases of increase in size toward the south, it can be shown that the forms have probably had a southern origin.

The Canidæe (wolves and foxes) have their largest representatives, the world over, in the north. "In North America the family is represented by six species, the smallest of which (speaking generally) are southern and the largest northern." The three species that have the widest ranges (the gray wolf, the common fox, and the gray fox) show the most marked differences in size. The skull, for instance, of "the common wolf is fully one-fifth larger in the northern parts of British America and Alaska than it is in northern Mexico, where it finds the southern limit of its habitat. Between the largest northern skull and the largest southern skull there is a difference of about thirty-five per cent of the mean size. Specimens from the intermediate region show a gradual intergradation between the extremes, although many of the examples from the upper Missouri country are nearly as large as those from the extreme north." The common fox is about one-tenth larger, on the arerage, in Alaska than it is in New England. The gray fox, whose habitat extends from Pennsylvania southward to Yucatan, has an average length of skull of about five inches in the north, and less than four in Central America - about ten per cent difference.

The Felidre, or cats, "reach their greatest development as respects both the number and the size of the species in the intertropical regions. This family has sent a single typical representative, the panther (Ficlis concolor), north of Mexico, and this ranges only to about the northern boundary of the United States. The other North $A$ merican representatives of the family are the lynxes, which in some of their varieties range from Alaska to Mexico." Although they vary greatly 
in different localities in color and in length and texture of pelage, they do not vary as to the size of their skulls. On the other hand the panther (and the ocelots) greatly increases in size southward, "or toward the metropolis of the family."

Other carnivora that increase in size northward are the badger, the marten, the fisher, the wolverine, and the ermine, which are all northern types.

Deer are also larger in the north; in the Virginia deer the annually deciduous antlers of immense size reach their greatest development in the north. The northern race of flying squirrels is one-half larger than the southern, "yet the two extremes are found to pass so gradually one into the other, that it is hardly possible to define even a southern and a northern geographical race." The species ranges from the arctic regions to Central America.

In birds also similar relations exist, but there is less often an increase in size northward. In species whose breeding station covers a wide range of latitude, the northern birds are not only smaller, but have quite different colors, as is markedly the case in the common quail, the meadow-lark, the purple grackle, the red-winged blackbird, the flicker, the towhee bunting, the Carolina dove, and in numerous other species. The same difference is also quite apparent in the blue jay, the crow, in most of the woodpeckers, in the titmice, numerous sparrows, and several warblers and thrushes. The variation often amounts to from ten to fifteen per cent of the average size of the species.

Allen also states that certain parts of the animal may vary proportionately more than the general size, there being an apparent tendency for peripheral parts to enlarge toward the warmer regions, i.c. toward the south. "In mammals which have the external ears largely developed - as in the wolves, foxes, some of the deer, and especially the hares - the larger size of this organ in southern as compared with northern individuals of the same species, is often strikingly apparent." 
It is even more apparent in species inhabiting open plains. The ears of the gray rabbit of the plains of western Arizona are twice the size of those of the Eastern states.

In birds the bill especially, but also the claws and tail, is larger in the south. In passing from New England southward to Florida the bill in slender-billed forms becomes larger, longer, more attenuated, and more decurved; while in short-billed forms the southern individuals have thicker and larger bills, although the birds themselves are smaller.

The remarkable changes and gradations of color in birds in different parts of North America are very instructive, and the important results obtained by American ornithologists form an interesting chapter in zoology. The evidence would convince the most sceptical of the difficulty of distinguishing between Linnean species. It is not surprising to find in this connection a leading ornithologist exclaiming, "if there really are such things as species." The differences here noted are mainly from east to west. We may briefly review here a few striking cases selected from Coues's "Key to North American Birds."

The flicker, or golden-winged woodpecker (Colaftes anratus), has a wide distribution in eastern North America. It is replaced in western North America (from the Rocky Mountains to the Pacific) by $C$. mericams. In the intermediate regions, Missouri and the Rocky Mountain region, the characters of the two are blended in every conceivable degree in different specimens. "Perhaps it is a hybrid, and perhaps it is a transitional form, and doubtless there are no such things as species in Nature. . . . In the west you will find specimens auratus on one side of the body, miricams on the other." There is a third form, $C$. chrysoides, with the wings and tail as in auratus, and the head as in mexicanus, that lives in the valley of the Colorado River, Lower California, and southward. 
In regard to the song-sparrow (Mclospiza), Cones writes: "The type of the genus is the familiar and beloved songsparrow, a bird of constant characters in the east, but in the west is split into numerous geographical races, some of them looking so clifferent from typical fasciata that they have been considered as distinct species, and even placed in other genera. This differentiation affects not only their color, but the size, relative proportions of parts, and particularly the shape of the bill; and it is sometimes so great, as in the case of $M$. cincrea, that less dissimilar looking birds are commonly assigned to different genera. Nevertheless the gradation is complete, and affected by imperceptible degrees. . . . The several degrees of likeness and unlikeness may be thrown into true relief better by some such expressions as the following, than by formal antithetical phrases: (I) The common eastern bird commonly modified in the interior into the duller colored (2) fallax. This in the Pacific watershed, more decidedly modified by deeper coloration, - broader black streaks in (3) hermanni, with its diminutive local race (4) samuclis, and more ruddy shades in (5) guttata northward, increasing in intensity with increased size in (6) rafina. Then the remarkable (7) cincrea, insulated much further apart than any of the others. A former American school would probably have made four 'good species,' (I) fasciata, (2) samuclis, (3) rafina, (4) cincrea."

Somewhat similar relations are found in three other genera of finches. Thus Passerella is "imperfectly differentiated "; Junco is represented by one eastern species, but in the west the stock splits up into numerous forms, "all of which intergrade with each other and with the eastern bird. Almost all late writers have taken a hand at Junco, shuffling them about in the vain attempt to decide which are 'species' and which 'varieties.' All are either or both, as we may elect to consider them." In the distribution of the genus Pipilo similar relations are found. There is an eastern form 
much more distinct from the western forms than these are from each other.

Finally may be mentioned the curious variations in screech-owls of the genus Scops. This owl has two strikingly different plumages - a mottled gray and a redclish brown, which, although very distinct when fully developed, yet " are entirely independent of age, season, or sex." There is an eastern form, Scops asio, that extends west to the Rocky Mountains. There is a northwestern form, S. kcmicotti, which in its red phase is quite different from S. asio, but in its gray plumage is very similar. The California form, S. benderii, is not known to have a red phase, and the gray phase is quite different from that of $S$. asio, but like the last form. The Colorado form, S. maxuclle, has no red phase, "but on the contrary the whole plumage is very pale, almost as if bleached, the difference evident in the nestlings even." The Texas form, S. masclli, has both phases, and is very similar to S. asio. The Florida form is smaller and colored like S. asio. The red phase is the frequent, if not the usual, one. The flammulated form, S. fiammula, is "a very small spcics, with much the general aspect of an ungrown S. asio." This is the southwestern form, easily distinguished on account of its small size and color from the other forms.

These examples might be greatly increased, but they will suffice, I think, to convince one of the difficulty of giving a sharp definition to "species." The facts speak strongly in favor of the transmutation theory, and show us how a species may become separated under different conditions into a $11 \mathrm{mm-}$ ber of new forms, which would be counted as new different species, if the intermediate forms were exterminated.

In discussing the nature of the changes that bring about variability, Darwin remarks: "From a remote period to the present day, under climates and circumstances as different as it is possible to conceive, organic beings of all kinds, when domesticated or cultivated, have varied. We see this with 
the many domestic races of quadrupeds and birds belonging to different orders, with goldfish and silkworms, with plants of many kinds, raised in various quarters of the world. In the deserts of northern Africa the date-palm has yielded thirty-eight varieties ; in the fertile plains of India it is notorious how many varieties of rice and of a host of other plants exist; in a single Polynesian island, twenty-four varieties of the breadfruit, the same number of the banana, and tiventytwo varieties of the arum, are cultivated by the natives. The mulberry tree of India and Europe has yielded many varieties serving as food for the silkworm; and in China sixtythree varieties of the bamboo are used for various domestic purposes. These facts, and innumerable others which could be added, indicate that a change of almost any kind in the conditions of life suffices to cause variability - different changes acting on different organisms."

Darwin thinks that a change in climate alone is not one of the potent causes of variability, because the native country of a plant, where it has been longest cultivated, is where it has oftenest given rise to the greatest number of varieties. He thinks it also doubtful that a change in food is an important source of variability, since the domestic pigeon has varied more than any other species of fowl, yet the food has been always nearly the same. This is also true for cattle and sheep, whose food is probably much less varied in kind than in the wild species.

Another point of interest is raised by Darwin. He thinks, as do others also, that the influence of a change in the conditions is cumulative, in the sense that it may not appear until the species has been subjected to it for several generations. Darwin states that universal experience shows that when new plants are first introduced into gardens they do not vary, but after several generations they will begin to vary to a greater or less extent. In a few cases, as in that of the dahlia, the zinnia, the Swan River daisy, and the 
Scotch rose, it is known that the new variations only appeared after a time. The following statement by Salter is then quoted, "Every one knows that the chief difficulty is in breaking through the original form and color of the species, and every one will be on the lookout for any natural sport, either from seed or branch; that being once obtained, however trifling the change may be, the result depends on himself." Jonghe is also quoted to the effect that "there is another principle, namely, that the more a type has entered into a state of variation, the greater is the tendency to continue doing so, and the more it has varied from the original type, the more is it disposed to vary still further." Darwin also quotes with approval the opinion of the most celebrated horticulturist of France, Vilmorin, who maintained that "when any particular variation is desired, the first step is to get the plant to vary in any manner whatever, and to go on selecting the most variable individuals, even though they vary in the wrong direction; for the fixed character of the species being once broken, the desired variation will sooner or later appear."

Darwin also cites a few cases where animals have changed quite quickly when brought under domestication. Turkeys raised from the eggs of wild species lose their metallie tints, and become spotted with white in the third generation. Wild ducks lose their true plumage after a few generations. "The white collar around the neck of the mallard becomes much broader and more irregular, and white feathers appear in the duckling's wings. They increase also in size of body." In these cases it appears that several generations were necessary in order to bring about a marked change in the original type, but the Australian dingoes, bred in the Zoological Gardens, produced puppies which were in the first generation marked with white and other colors.

The following cases from De Varigny are also very striking. The dwarf trees from Japan, for the most part conifers, which 
may be a hundred years old and not be more than three feet high, are in part the result "of mechanical processes which prevent the spreading of the branches, and in part of a starving process which consists in cutting most roots and in keeping the plant in poor soil."

As an example of the sudden appearance of a new variation the following case is interesting. A variety of begonia is recorded as having appeared quite suddenly at a number of places at the same time. In another case a narcissus which had met with adverse circumstances, and had then been supplied with a chemical manure in some quantity, began to bear double flowers.

Amongst animals the following cases of the appearance of sudden variations are pointed out by De Varigny. "In Paraguay, during the last century (1770), a bull was born without horns, although his ancestry was well provided with these appendages, and his progeny was also hornless, although at first he was mated with horned cows. If the horned and the hornless were met in fossil state, we would certainly wonder at not finding specimens provided with semi-degenerate horns, and representing the link between both, and if we were told that the hornless variety may have arisen suddenly, we should not believe it and we should be wrong. In South America also, between the sixteenth and eighteenth centuries the niata breed of oxen sprang into life, and this breed of bulldog oxen has thriven and become a new race. So in the San Paulo provinces of Brazil, a new breed of oxen suddenly appeared which was provided with truly enormous horns, the breed of franqueiros, as they are called. The mauchamp breed of sheep owes its origin to a single lamb that was born in I 828 from merino parents, but whose wool, instead of being curly like that of its parents, remained quite smooth. This sudden variation is often met with, and in France has been noticed in different herds."

The ancon race of sheep originated in I79I from a ram 
born in Massachusetts having short crooked legs and a long back. From this one ram by crossing, at first with common sheep, the ancon race has been produced. "When crossed with other breeds the offspring, with rare exception, instead of being intermediate in character, perfectly resemble either parent; even one of twins has resembled one parent and the second the other."

Two especially remarkable cases remain to be described. These are the Porto Santo rabbit and the japanned peacock. Darwin has given a full account of both of these cases. "The rabbits which have become feral on the island of Porto Santo, near Madeira, deserve a fuller account. In I4I 8 or I 4 I9 J. Gonzales Zarco happened to have a female rabbit on board which had produced young during the voyage, and he turned them all out on the island. These animals soon increased so rapidly that they became a nuisance, and actually caused the abandonment of the settlement. Thirty-seven years subsequently, Cada Mosto describes them as innumerable; nor is this surprising, as the island was not in habited by any beast of prey, or by any terrestrial mammal. We do not know the character of the mother rabbit; but it was probably the common domestic kind. The Spanish peninsula, whence Zarco sailed, is known to have abounded with the common wild species at the most remote historical period; and as these rabbits were taken on board for food, it is improbable that they should have been of any peculiar breed. That the breed was well domesticated is shown by the doe having littered during the voyage. Mr. Wollaston, at my request, brought two of these feral rabbits in spirits of wine; and, subsequently, Mr. W. Haywood sent home three more specimens in brine and two alive. These seven specimens, though caught at different periods, closely resemble each other. They were full-grown, as shown, by the state of their bones. Although the conditions of life in Porto Santo are evidently highly favorable to rabbits, as proven by their extraordinarily rapict 
increase, yet they differ conspicuously in their small size from the wild English rabbit. . . In color the Porto Santo rabbit differs considerably from the common rabbit; the upper surface is redder, and is rarely interspersed with any black or black-tipped hairs. The throat and certain parts of the under surface, instead of being pure white, are generally gray or leaden color. But the most remarkable difference is in the ears and tail. I have examined many fresh English rabbits, and the large collection of skins in the British Museum from various countries, and all have the upper surface of the tail and the tips of the ears clothed with blackish gray fur; and this is given in most works as one of the specific characters of the rabbit. Now in the seven Porto Santo rabbits the upper surface of the tail was reddish brown, and the tips of the ears had no trace of the black edging. But here we meet with a singular circumstance: in June, I86I, I examined two of these rabbits recently sent to the Zoological Gardens and their tails and ears were colored as just described; but when one of their dead bodies was sent to me in February, I 863, the ears were plainly edged, and the upper surface of the tail was covered with blackish gray fur, and the whole body was much less red; so that under the English climate this individual rabbit had recovered the proper color of its fur in rather less than four years."

Another striking case of sudden variation is found in the peacock. It is all the more remarkable because this bird has hardly varied at all under domestication, and is almost exactly like the wild species living in India to-day. Darwin states: "There is one strange fact with respect to the peacock, namely, the occasional appearance in England of the 'japanned' or 'black-shouldered' kind. This form has lately been named, on the high authority of Mr. Slater, as a distinct species, viz. Paro nigripennis, which he believes will hereafter be found wild in some country, but not in India, where it is certainly unknown. The males of these japanned birds 
differ conspicuously from the common peacock in the color of their secondary wing-feathers, scapulars, wing-coverts, and thighs, and are, I think, more beautiful; they are rather smaller than the common sort, and are always beaten by them in their battles, as I hear from the Hon. A. S. G. Canning. The females are much paler-colored than those of the common kind. Both sexes, as Mr. Canning informs me, are white when they leave the egg, and they differ from the young of the white variety only in having a peculiar pinkish tinge on their wings. These japanned birds, though appearing suddenly in flocks of the common kind, propagate their kind quite truly."

In two cases, in which these birds had appeared quite suddenly in flocks of the ordinary kind, it is recorded that "though a smaller and weaker bird, it increased to the extinction of the previously existing breed." Here we have certainly a remarkable case of a new species suddenly appearing and replacing the ordinary form, although the birds are smaller, and are beatcn in their battles.

Darwin has given an admirably clear statement of his opinion as to the causcs of arability in the opening paragraph of his chapter dealing with this topic in his "Animals and Plants." Some authors, he says, "look at variability as a necessary contingent on reproduction, and as much an original law as growth or inheritance. Others have of late encouraged, perhaps unintentionally, this view by speaking of inheritance and variability as equal and antagonistic principles. Pallas maintained, and he has had some followers, that variability depends exclusively on the crossing of primordially distinct forms. Other authors attribute variability to an excess of food, and with animals, to an excess relatively to the amount of exercise taken, or again, to the effects of a more genial climate. That these causes are all effective is highly probable. But we must, I think, take a broader view, and conclude that organic beings, when subjected during several generations to 
any change whatever in their condition, tend to vary; the kind of variation which ensues depending in most cases in a far higher degree on the nature of the constitution of the being, than on the nature of the changed conditions."

Most naturalists will agree, in all probability, with this conclusion of Darwin's. The examples cited in the preceding pages have shown that there are several ways in which the organisms may respond to the environment. In some cases it appears to affect all the individuals in the same way; in other cases it appears to cause them to fluctuate in many directions; and in still other cases, without any recognizable change in the external conditions, new forms may suddenly appear, often of a perfectly definite type, that depart widely from the parent form.

For the theory of evolution it is a point of the first importance to determine which of these modes of variation has supplied the basis for evolution. Moreover, we are here especially concerned with the question of how adaptive variations arise. Without attempting to decide for the present between these different kinds of variability, let us examine certain cases in which an immediate and adaptive response to the environment has been described as taking place.

Responsive Changes in the Organism that adapt it to the New Environment

There is some experimental evidence showing that sometimes organisms respond directly and adaptively to certain changes in the environment. Few as the facts are, they require very careful consideration in our present examination. The most striking, perhaps, is the acclimatization to different temperatures. It has been found that while few active organisms can withstand a temperature over 45 degrees $C$., and that for very many 40 degrees is a fatal point, yet, on the other hand, there are organisms that live in certain hot springs 
where the temperature is very high. Thus, to give a few examples, there are some of the lower plants, nostocs and protococcus forms, that live in the geysers of California at a temperature of 93 degrees C., or nearly that of boiling water. Leptothrix is found in the Carlsbad springs, that have a temperature of 44 to 54 degrees. Oscillaria have been found in the Yellowstone Park in water between 54 and 68 degrees, and in the hot springs in the Philippines at $7 \mathrm{I}$ degrees, and on Ischia at 85 degrees, and in Iceland at 98 degrees.

It is probable from recent observations of Setchel that most of the temperatures are too high, since he finds that the water at the edge of hot springs is many degrees lower than that in the middle parts.

The snail, Phy'sa acuta, has been found in France living at a temperature of 35 to 36 degrees; another snail, Paludina, at Abano, Padua, at 50 degrees. Rotifers have been found at Carlsbad at 45 to 54 degrees; Anguillidae at Ischia at 8 I degrees; Cypris balncaria, a crustacean at Hammam-Neckhoutin, at 8 I clegrees; frogs at the baths of "Pise" at $3 S$ degrees.

Now, there can be little doubt that these forms have had ancestors that were like the other members of the group, and would have been killed had they been put at once into water of these high temperatures, therefore it seems highly probable that these forms have become specially adapted to live in these warm waters. It is, therefore, interesting to find that it has been possible to acclimatize animals experimentally to a temperature much above that which would be fatal to them if subjected directly to it. Dutrochet (in I 8 I 7 ) found that if the plant, nitella, was put into water at 27 clegrees, the currents in the protoplasm were stopped, but soon began again. If put now into water at 34 degrees they again stopped moving, but in a quarter of an hour began once more. If then put into water at 40 degrees the currents again slowed down, but began again later.

Dallinger (in I880) made a most remarkable series of ex- 


\section{External and Internal Factors in Evolution $32 \mathrm{I}$}

periments on flagellate protozoans. He kept them in a warm oven, beginning at first at a temperature of I 6.6 degrees C. "He employed the first four months in raising the temperature 5.5 degrees. This, however, was not necessary, since the rise to $2 \mathrm{I}$ degrees can be made rapidly, but for success in higher temperatures it is best to proceed slowly from the beginning. When the temperature had been raised to 23 degrees, the organisms began dying, but soon ceased, and after two months the temperature was raised half a degree more, and eventually to 25.5 degrees. Here the organisms began to succumb again, and it was necessary repeatedly to lower the temperature slightly, and then to advance it to 25.5 degrees, until, after several weeks, unfavorable appearances ceased. For eight months the temperature could not be raised from this stationary point a quarter of a degree without unfavorable appearances. During several years, proceeding by slow stages, Dallinger succeeded in raising the organisms up to a temperature of 70 degrees C., at which the experiment was encled by an accident." 1

Davenport and Castle carried out a series of experiments on the egg of the toad, in which they tried to acclimatize the eggs to a temperature higher than normal. Recently laid eggs were used; one lot kept at a temperature of $\mathrm{I}_{5}$ degrees C., the other at 24-25 degrees C. Both lots developed normally. At the end of four weeks the temperature point at which the tadpoles were killed was determined. Those reared at a temperature of $\mathrm{I} 5$ degrees C. died at $4 \mathrm{I}$ degrees C., or below; those reared at 24-25 degrees C. sustained a temperature ro degrees higher; no tadpole dying in this set under 43 degrees C. "This increased capacity for resistance was not produced by the dying off of the less resistant individuals, for no death occurred in these experiments during the gradual elevation of the temperatures in the cultures." The increased resistance was due, therefore,

1 Quoted from Davenport's " Experimental Morphology." 
to a change in the protoplasm of the individuals. It was also determined that the acquired resistance was only very gradually lost (after seventeen days' sojourn in cooler water). The explanation of this result may be clue, in part, to the protoplasm containing less water at higher temperatures, for it is known that while the white of egg (albumen) coagulates at 56 degrees $\mathrm{C}$. in aqueous solution; with only i 8 per cent of water it coagulates between 80 degrees and 90 degrees C. ; and with 6 per cent, at I 45 degrees C.; and without water between 100 degrees and I 70 degrees $\mathrm{C}$.

It has long been known that organisms in the dry condition resist a much higher temperature. The damp uredospore is killed at 58.5 degrees to 60 degrees C.; but dry spores withstand 128 degrees $\mathrm{C}$. It is also known that organisms may become acclimatized to cold through loss of water, but we lack exact experimental data to show to what extent this can be carried.

There are also some experiments that go to show that animals may become attuned to certain amounts of light, but the facts in this connection will be described in another chapter.

Some important results have been obtained by accustoming organisms to solutions containing various amounts of salts. A number of eases of this sort are given by De Varigny. It has been found that littoral marine animals that live where the water may become diluted by the rain, or by rivers, survive better when put into fresh water than do animals living farther from the shore. Thus the oyster, the mussel, and the snail, Patella, withstand immersion in fresh water better than other animals that live farther out at sea. The reverse is also true; fresh-water forms, such as Lymnaa, Physa, Paludina, and others may be slowly acclimatized to water containing more salt. The forms mentioned above could be brought by degrees into water containing 4 per cent of salt, which would have killed the animals if they had been brought suddenly into it. Similar results have been obtained for amoba. 
It has been shown that certain rotifers and tardigrades, and also some unicellular animals, that live in pools and ponds that are liable to become dry, withstand desiccation, while other members of the same groups, living in the sea, do not possess this power of resistance. Cases of this sort are usually explained as cases of adaptation, but it has not been shown experimentally that resistance to drying can be acquired by a process of acclimatization to this condition. The case is also in some respects different from the preceding, since intermediate conditions are less likely to be met with, or to be of sufficiently long duration for the animal to become acclimatized to them. It seems more probable, in such cases, that these forms have been able to live in such precarious conditions from the beginning because they could resist the effects of drying, not that they have slowly acquired this power. Finally, there must be discussed the question of the acclimatization to poisons, to which an individual may be rendered partially immune. The point of special importance in this connection is that the animal may be said to respond adaptively to a large number of substances, which it has never met before in its individual history, or to which its ancestors have never been subjected. It may become slowly adapted to many different kinds of injurious substances. These cases are amongst the most important adaptive individual responses with which we are familiar, and the point cannot be too much emphasized that organisms have this latent capacity without ever having had an opportunity to acquire it through experience.

The preceding groups of phenomena, included under the general heading of individual acclimatization, have one striking thing in common, namely, that a physiological adaptation is brought about without a corresponding change in form, although we must suppose that the structure has been altered in certain respects at least. The form of the individual remains the same as before, but so far 
as its powers of resistance are concerned it is a very different being.

In regard to the perpetuation of the advantages gained by means of this power of adaptation, it is clear in those cases in which the young are nourished during their embryonic life by the mother, that, in this way, the young may be rendered immune to a certain extent, and there are instances of this sort recorded, especially in the case of some bacterial diseases. Whether this power can also be transmitted through the egg, in those instances in which the egg itself is set free and development takes place outside the body, has not been shown. In any case, the effect appears not to be a permanent one and will wear off when the particular poison no longer acts. It is improbable, therefore, that any permanent contribution to the race could be gained in this way. Adaptations of this sort, while of the highest importance to the individual, can have produced little direct effect on the evolution of new forms, although it may have been often of paramount importance to the individuals to be able to adapt themselves, or rather to become able to resist the effect of injurious substances. The important fact in this connection is the wonderful latent power possessed by all animals. So many, and of such different kinds, are the substances to which they may become immune, that it is inconceivable that this property of the organism could ever have been acquired through experience, no matter how probable it may be made to appear that this might have occurred in certain cases of fatal bacterial discases. And if not, in so many other cases, why invent a special explanation for the few cases?

We may defer the general discussion of the rôle that external factors have played in the adaptation of organisms, until we have examined some of the theories which attribute changes to internal factors. The idea that something innate in the living substance itself has served as the basis for evolu- 
tion has given rise to a number of different hypotheses. That of the botanist Nägeli is one of the most elaborately worked out theories of this sort that has been proposed, and may be examined by way of illustration.

\section{Nägeli's Perfecting Principle}

Nägeli used the term complcting principle ("Vervollkom. mungsprincip") to express a tendency toward perfection and specialization. Short-sighted writers, he says, have pretended to see in the use of this principle something mystical, but on the contrary it is intended that the term shall be employed in a purely physical sense. It represents the law of inertia in the organic realm. Once set in motion, the developmental process cannot stand still, but must advance in its own direction. Perfection, or completion, means nothing else than the advance to complicated structure, "but since persons are likely to attach more meaning to the word perfection than is intended, it would perhaps be better to replace it with the less objectionable word progression."

Nägeli says that Darwin, having in view only the condition of adaptation, designates that as more complete which gives its possessor an advantage in the battle for existence. Nägeli claims that this is not the only criterion that applies to organisms, and it leaves out the most important part of the phenomenon. There are two kinds of completeness which we should keep distinctly apart: (I) the completeness of organization characterized by the complication of the structure and the most far-reaching specialization of the parts; (2) the completeness of the adaptation, present at each stage in the organization, which consists in the most advantageous development of the organism (under existing conditions) that is possible with a given complication of structure and a given division of functions. 
The first of these conceptions Nägeli always calls "completeness" (Vollkommenheit), for want of a simpler and better expression; the second he calls adaptation. By way of illustrating the difference between the two, the following examples may be given. The unicellular plants and the moulds are excellently adapted each to its conditions of life, but they are much less complete in structure than an apple tree, or a grape vine. The rotifers and the leeches are well adapted to their station, but in completeness of structure they are much simpler than the vertebrates.

If we consider only organization and division of labor as the work of the completing principle, and leave for the moment adaptation out of account, we may form the following picture of the rise of the organic world. From the inorganic world there arose the simplest organic being thinkable, being little more than a drop of substance. If this underwent any change at all, it would have been necessarily in the direction of greater complication of structure; and this would constitute the first step in the upward direction. In this way Nägeli imagines the process once begun would continue. When the movement has reached a certain point, it must continue in the same direction. The organic kingdom consists, therefore, of many treelike branches, which have had a common startingpoint. Not only does he suppose that organisms were once spontaneously generated, and began their first upward course of development, but the process has been repeated over and over again, and each time new series have been started on the upward course. The organic kingdom is made up, therefore, of all degrees of organization, and all these have had their origins in the series of past forms that arose and began their upward course at different times in the past. Those that are the highest forms at the present time represent the oldest series that successfully developed; the lowest forms living at the present time are the last that have appeared on the scene of action. 
Organisms, as has been said, are distinguished from one another, not only in that one is simpler and another more complicated, but also in that those standing at the same stage of organization are unequally differentiated in their functions and in their structure, which is connected primarily with certain external relations which Nägeli calls adaptations.

Adaptation appears at each stage of the organization, which stage is, for a given enviromment, the most advantageous expression of the main type that was itself produced by internal causes. For this condition of adaptation, a sufficient cause is demanded, and this is, as Nägeli tries to show later, the result of the inherited response to the environment. In many cases this cause will continue to act until complete adaptation is gained; in other cases, the external conditions give a direction only, and the organism itself continues the movement to its more perfect condition.

The difference between the conception of the organic kingdom as the outcome of mechanical causes on the one hand, or of competition and extermination on the other hand, can be best brought out, Nägeli thinks, by the following comparison of the two respective methods of action. There might have been no competition, and no consequent extermination in the plant kingdom, if from the beginning the surface of the earth had continually grown larger in proportion as living things increased in numbers, and if animals had not appeared to destroy the plants. Under these conditions each germ cou,d then have found room and food, and have unfolded itself without hinderance. If now, as is assumed to be the case on the Darwinian theory, individual variations had been in all directions, the developmental movement could not have gone beyond its own beginnings, and the first-formed plants would have remained swinging now on one side and now on another of the point first reached. The whole plant kingdom would have remained in its entirety at its first stage of evolution, that is, it would never have advanced beyond the stage 
of a naked drop of plasma with or without a membrane. But, according to the further Darwinian conception, competition, leading to extermination, is capable of bringing such a condition to a higher stage of development, since it is assumed that those individuals which vary in a beneficial direction would have an advantage over those that have not taken such a step, or have made a step backward.

If, on the other hand, under the above-mentioned conditions of unrestricted development, without competition, variations were determined by " mechanical principles," then, according to Nägeli's view, all plant forms that now exist would still have evolved, and would be found living at the present time, but along with all those that now exist there would be still other forms in countless numbers. These would represent those forms which have been suppressed. On Nägeli's view competition and suppression do not produce new forms, but only weed out the intermediate forms. He says without competition the plant kingdom would be like the Milky Way; in consequence of competition the plant kingdom is like the firmament studded with bright stars.

The plant kingdom may also be compared to a branched tree, the ends of whose branches represent living species. This tree has an inordinate power of growth, and if left to itself it would produce an impenetrable tangle of interwoven branches. The gardener prevents this crowding by cutting away some of the parts, and thus gives to the tree distinct branches and twigs. The tree would be the same without the watchful trimming of the gardener, but without definite form.

Nägeli states: "From my earlier researches I believe that the external influences are small in comparison to the internal ones. I shall speak here only of the influences of climate and of food, which are generally described as the causes of change, without however any one's having really determined whether or not a definite result ean be brought about by these factors. Iater I shall speak of a special class of external influences 
which, according to my view, bring forth beyond a doubt adaptive changes."

The external influence of climate and of food act only as transitory factors. A rich food supply produces fat, lack of food leads to leanness, a warm summer makes a plant more aromatic, and its fruit sweeter; a cold year means less odor and sour fruit. Of two similar seeds the one sown in rich soil will produce a plant with many branches and abundance of flowers; the other, planted in sandy soil, will produce a plant without branches, with few flowers, and with small leaves. The seeds from these two plants will behave in exactly the same way; they have inherited none of the differences of their parents. Influences of this sort, even if extending over many generations, have no permanent effect. Alpine plants that have lived since the ice age under the same conditions, and have the characters of true highmountain plants, lose these characters completely during the first summer, if transplanted to the plains. Moreover, it makes no difference whether the seed or the whole plant itself be transferred. In place of the dwarfed, unbranched growth, and the reduced number of organs, the plant when transferred to the plains shoots up in height, branches strongly, and produces numerous leaves and flowers. The plants retain their new characters as long as they live in the plain without any other new variation being observed in them.

Other characteristics also, which arise from different kinds of external influences due to different localities, such as dampness and shade, a swampy region, or different geological substrata, last only so long as the external conditions last.

These transient peculiarities make up the characters of local varieties. That they have no permanency is intelligible, since they exhibit no new characters, but the change consists mainly in the over- or under-development of those peculiarities that are dependent on external influences. The effect of these influences may be compared to an elastic rod, 
which, however much it may be distorted by external circumstances, returns again to its original form as soon as released.

Besides these temporary changes, due to external influences, there are many cases known in which the same plant lives under very diverse conditions and yet remains exactly the same. For example, the species of Rhododendron forragincum lives on archæan mountains and especially where the soil is poor in calcium. Another species, Rhododendron hirsutum is found especially on soil rich in calcium. The difference in the two species has been supposed to depend on differences in the soil, and if so, we would imagine that, if transplanted for a long time, the one should change in the direction of the other. Yet it is known that the rusty rhododendron may be found in all sorts of localities, even on dry, sunny, calcareous rocks of the Apennines and of the Jura, and despite its residence in these localities, since the glacial epoch, no change whatever has taken place.

Single varieties of the large and variable genus of Hicracium have lived since the glacial period in the high regions of the Alps, Carpathians, and in the far north, and also in the plains of different geological formations, but these varieties have remained exactly the same, although on all sides there are transitional forms leading from these to other varieties.

Some parasitic species also furnish excellent illustrations of the same principle. Besides the several species of Orobanchia and of the parasitic moulds, the mistletoe deserves special mention. It lives on both birch and apple trees and on both presents exactly the same appearance; and even if it is true that mistletoe growing on conifers presents certain small deviations in its character, it is still doubtful whether, if transferred to the birch or apple tree, it would not lose these differences, thus indicating that they are not permanent.

It is a fact of general observation that, on the one hand, the same variety occurs in different localities and under different surroundings, and, on the other hand, that slightly 
different varieties live together in the same place and therefore under the same external conditions. It is evident, then, that food conditions have neither originated the differences nor kept them up. The rarer cases in which in different localities different varieties exist show nothing, because competition and suppression keep certain varieties from developing where it would be possible otherwise for them to exist.

Nägeli says his conclusion may be tested from another point of view. If food conditions, as is generally supposed, have a definite, i.c. a permanent, effect on the organism, then all organisms living under the same conditions should show the same characters. Indeed, it has been claimed in some instances that this is actually the case. Thus it is stated that dry localities cause plants to become hairy, and that absence of hairiness is met with in shady localities. This may apply to certain species, but in other cases exactly the reverse is true, and even the same species behaves differently in different regions, as in Hicracium. And so it is with all characteristics which are ascribed to external influences. As soon as it is supposed a discovery has been made in this direction, we may rest assured that in other cases the reverse will be found to hold. We have had, in respect to the influence of the outer world on organisms, the same experience as with the rules for the weather, - when we come to examine the facts critically there are found to be as many exceptions as confirmations of the rule.

If climatic influence has a definite effect, the entire flora of a special locality ought to have the same peculiarities, but this stands in contradiction to all the results of experience. The character of the vegetation is not determined by the environment of the plants but by their prehistoric origin, and as the result of competition. Nägeli concludes his discussion with the statement that all of our experience goes to show that the effects of external influences (climate and food) 
appear at once, and their results last only as long as the influences themselves last, and are then lost, leaving nothing permanent behind. This is true even when the external influences have lasted for a long time, - since the glacial epoch, for instance. We find, he claims, nothing that supports the view that such influences are inherited.

If we next examine the question of changes from internal causcs, Nägeli claims that here also observation and research fail to show the origin of a new species, or even of a new variety from external causes. In the organic world little change has taken place, he believes, since the glacial epoch. Many varieties have even remained the same throughout the whole intervening time; and while it cannot be doubted that new varieties have also been formed, yet the cause of their origin cannot be empirically demonstrated. The permanent, hereditary characters, of whose origin we know something from experience, belong to the individual changes which have appeared under cultivation in the formation of domestic races. These are for the most part the result of crossing. So far as we have any definite information as to the origin of the changes, they are the result of inner, and never of external, causes. We recognize that this must be the case, since under the same external conditions individuals behave differently - in the same flower-bud some seeds give rise to plants like the parent, others to altered ones. The strawberry with a single leaflet, instead of three, arose in the last century in a single individual amongst many other ordinary plants. From the ten seeds of a pear Van Mons obtained as many different kinds of pears. The most conclusive proof of the action of inner causes is most elearly seen when the branches of the same plant differ. In Geneva a horse-chestnut bore a branch with "filled" flowers, and from this branch, by means of cuttings, this variation has been carried over all Europe. In the Botanic Garden at Munich there is a beech with small divided leaves; but one of its branches produces 
the common broad undivided leaves. Many such examples have been recorded which can only be explained by assuming that a cell, or a group of cells, like those from which the other branches arose, have become changed in some unknown way as the result of inner causes. The properties that are permanent and inherited are contained in the idioplasm, which the parent transmits to its offspring. A cause that permanently transforms the organism must also transform the idioplasm. How powerless, in comparison to internal causes, the external causes are is shown most conclusively in grafting. The graft, although it receives its nourishment through the stock, which may be another species, remains itself unchanged.

Nägeli makes the following interesting comparison between the development of the individual from an egg, and the evolution, or development, of the phylum. No one will doubt that the egg during the entire time of its process of transformation is guided by internal factors. Each successive stage follows with mechanical necessity from the preceding. If an animal can develop from inner causes from a drop of plasma, why should not the entire evolutionary process have also been the outcome of developmental inner causes? He admits that there is a difference in the two cases in that the plasma that forms the egg has come from another animal, and contains all the properties of the individual in a primordial condition. In the other case we must suppose that the original drop of plasma did not contain at first the primordium of definite structures, but only the ability to form such. Logically the difference is unimportant. The main point is that in the primordium of the germ a special peculiarity of the substance is present which by forming new substances grows, and changes as it grows, and the one change of necessity excites the next until finally a highly organized being is the result.

Nägeli discusses a question in this connection, which, he says, has been unnecessarily confused in the descent theory. 
Since we are entirely in the dark as to how much time has been required for the formation of phyla, so also are we ignorant as to how long it may have taken for each step in advance. We may err equally in ascribing too much and too little time to the process. It is, moreover, not necessary that for every step the same amount of time should have been required. On the contrary, the probability is that recognizable changes may at times follow each other rapidly, and then for a time come to a standstill, - just as in the development of the individual there are periods of more rapid and others of less rapid change.

A more difficult problem than that relating to the sort of changes the external influences bring about in the organism, is the question as to how they effect the organism, or how they act on it mechanically. This, as is well known, was answered by Darwin, who regards all organization as a problem of adaptation: only those chance variations surviving which are capable of existence, the others being destroyed. On this theory external influences have only a negative or a passive action, namely, in setting aside the unadapted individuals. Nägeli, on the other hand, looks upon some kinds of external conditions as directly giving rise to the adaptive characters of the organism. This is accomplished, he supposes, in the following ways: two kinds of influence are recognized; the dirct action, which, as in inorganic nature, comes to an end when the external influences come to an end, as when cold diminishes the chemical actions in the plant; and the indircet action, generally known as a stimulus, which starts a series of molecular motions, invisible to us, but which we recognize only in their effects. Very often the stimulus starts only a reflex action, usually at the place of application.

A stimulus acting for but a short time produces no lasting effect on the idioplasm. A person stung by a wasp suffers no permanent effect from the injury. But if a stim- 
ulus acts for a long time, and through a large number of generations, then it may, even if of small strength, so change the idioplasm, that a tendency or disposition capable of being seen may be the result. This appears to be the case in regard to the action of light, which causes certain parts of the plant to turn toward it and others away from it; also for the action of gravity, which determines the downward direction of the roots. It may be claimed, perhaps, that these are the results of direct influence and not of an internal response, but this is not the case; for some plants act in exactly the opposite way, and send a stem downward, as in the case of the cleistogamous flowers of Cardamine chenopodifolia; and other plants turn away from the light. This means that the idioplasm behaves differently in different plants in response to the same stimulus.

Concerning the more visible effects of adaptation, Nägeli states that in regard to some of them there can be no question as to how they must have arisen. Protection against cold, by the formation of a thick coat of hair, is the direct result of the action of the cold on the skin of the animal. The different weapons of offence and of defence, horns, spurs, tusks, etc., have arisen, he maintains, through stimulus to those parts of the body where these structures arise.

The causes of the other adaptations, especially of those occurring in plants, are less obvious. Land plants protect themselves from drying by forming a layer of cork over the surface. The most primitive plants were water plants, which acclimated themselves little by little to moist, and then to dry, air. When they first emerged from the water the drying acted as a stimulus on the surface, and caused it to harden in the same way as a drop of glue hardens. This hardening in turn acted as a stimulus, causing a chemical transformation of the surface into a corky substance. This effect was inherited, and in this way the power to form cork originated. 
Land plants have, in addition to the soft parts, the hard bast and wood which serves the mechanical purpose of supporting the soft tissues and protecting them from being injured. The arrangement of the hard parts is such as to suggest that they are the result of the action of pressures and tensions on the plant, for the strongest cells are found where there is most need for them. It is easy to imagine, Nägeli adds, that this important arrangement of the tissues is the result of external forces which brought about the result in these parts.

Nägeli accounts for the origin of twining plants as follows. Being overshadowed by other plants, the stem will grow rapidly in the damp air. Coming in contact with the stems of other plants, the delicate stem is stimulated on one side, and grows around the point of contact. This tendency becomes inherited, and the habit to twine is ultimately established.

The difference in the two sides of leaves is explained by Nägeli as the result of the difference in the illumination of the two sides. This influence of light on the leaf has been inherited. The formation of the tubular corolla that is seen in many plants visited by insects is explained as the result of the stimulus produced by the insects in looking for the pollen. The increase in the length of the proboscis of the insect is the result of the animal straining to reach the bottom of the ever elongating tube of the corolla. "The tubular corolla and the proboscis of the insect appear as though made for each other. Both have slowly developed to their present condition, the long tube from a short tube and the long proboscis from a short one." Thus, by purely Lamarckian principles, Nägeli attempts to account for many of the adaptations between the organism and the outer world. But if this takes place, where is there left any room for the action for his so-called perfecting principle? Nägeli proceeds to show how he supposes that the two work together. 
As a result of inner causes the organism would pass through a series of perfectly definite stages, $\mathrm{J}, \mathrm{J}^{1}, \mathrm{~J}^{2}$. But if, at any stage, external influences produced an effect on the organism so that the arrangement of the idioplasm changes in response, a new adaptation is produced. In this way new characters, not inherent in the idioplasm, may be added, and old ones be changed or lost. "In order not to be misunderstood in regard to the completing or perfecting principle I will add, that I ascribe to it no determinate action in the organism, neither in producing the long neck of the giraffe, nor the prehensile tail of the ape, neither the claws of the crab, nor the decoration of the bird of paradise. These structures are the outcome of both factors. I cannot picture to myself how external causes alone, and just as little how internal causes alone, could have changed a monad into a man." But Nägeli goes on to say, that if at any stage of organization one of the two causes should cease to act, the other could only produce certain limited results. Thus, if external causes alone acted, the organization would remain at the same stage of completeness, but might become adapted to all kinds of external conditions - a worm, for instance, would not develop into a fish, but would remain a worm forever, although it might change its worm structure in many ways in response to external stimuli. If, on the other hand, only the completing principle acted, then without changing its adaptations the number of the cells and the size of the organs might be increased, and functions that were formerly united might become separated. Thus, without altering the character of the organism, a more highly developed (in the sense of being more specialized) organism would appear.

Nägeli, as we have just seen, has attempted to build up a conception of nature based on two assumptions, neither of which has been demonstrated to be an actual principle of development. His hypothesis appears, therefore, entirely arbitrary and speculative to a high degree. Even if it were 
conceivable that two such principles as these control the evolution of organisms, it still requires a good deal of imagination to conceive how the two go on working together. Moreover, it is highly probable that whole groups have evolved in the direction of greater simplification, as seen especially in the case of those groups that have become degenerate. To what principle can we refer processes of this sort?

It is certainly a strange conclusion this, at which Nägeli finally arrives, for, after strenuously combating the idea that the external factors of climate and of food have influence in producing new species, he does not hesitate to ascribe all sorts of imaginary influences to other external causes. The apparent contradiction is due, perhaps, to the fact that his experience with actual species led him to deny that the direct action of the environment produces permanent changes, while in theory he saw the necessity of adding to his perfecting principle some other factor to explain the adaptations of the new forms produced by inner causes. Nägeli seems to have felt strongly the impossibility of explaining the process of evolution and of adaptation as the outcome of the selection of chance variations, now in this direction, now in that. He seems to have felt that there must be something within the organism that is driving it ever upward, and he attempts to avoid the teleological element, which such a conception is almost certain to introduce, by postulating the inheritance of the effects of long-continued action of the environment, in so far as certain factors in the environment produce a response in the organism. Nevertheless, this combination is not one that is likely to commend itself, aside from the fact that the assumptions have no evidence to support them. Despite Nägeli's protest that his principles are purely plysical, and that there is nothing mystical in his point of view, it must be admitted that his conception, as a whole, is so vague and difficult in its application that it probably deserves the neglect which it generally receives. 
Nägeli's wide experience with living plants convinced him that there is something in the organism over and beyond the influence of the external world that causes organisms to change; and we cannot afford, I think, to despise his judgment on this point, although we need not follow him to the length of supposing that this internal influence is a "force" driving the organism forward in the direction of ever greater complexity. A more moderate estimate would be that the organism often changes through influences that appear to us to be internal, and while some of the changes are merely fluctuating or chance variations, there are others that appear to be more limited in number, but perfectly definite and permanent in character. It is the latter, which, I believe, we can safely accredit to internal factors, and which may be compared to Nägeli's internal causes, but this is far from assuming that these changes are in the direction of greater completeness or perfection, or that evolution would take place independently of the action of external agencies. 


\section{CHAPTER X}

THE ORIGIN OF THE DIFFERENT KINDS OF ADAPTATIONS

In the present chapter we may first consider, from the point of view of discontinuous variations as contrasted with the theory of the selection of individual variations, the structural adaptations of animals and plants, i.e. those cases in which the organism has a definite form that adapts it to live in a particular environment. In the second place, we may consider those adaptations that are the result of the adjustment of each individual to its surroundings. In subsequent chapters the adaptations connected with the responses of the nervous system and with the process of sexual reproduction will be considered.

It should be stated here, at the outset, that the term mutation will be used in the following chapters in a very general way, and it is not intended that the word shall convey only the idea which De Vries attaches to it; it is used rather as synonymous with discontinuous and also definite iariation of all kinds. The term will be used to include "the single variations" of Darwin, "sports," and even orthogenic variation, if this has been definite or discontinuous.

\section{Form AND Symmetry}

Almost without exception, animals and plants have definite and characteristic forms. In other worls, they are not amorphous masses of substance. The members of each species conform, more or less, to a sort of icleal type. Our 
first problem is to examine in what sense the form itself may be looked upon as an adaptation to the surroundings.

It is a well-recognized fact that the forms of many animals appear to stand in a definite relation to the environment. For instance, animals, that move in definite directions in relation to their structure have the anterior and the posterior ends quite different, and it is evident that these ends stand in quite different relations to surrounding objects; while, on the other hand, the two sides of the body which are, as a rule, subjected to the same influences are nearly exactly alike. The dorsal and the ventral surfaces of the body are generally exposed to very different external conditions, and are quite different in structure.

The relation is so obvious in most cases that it might lead one quite readily to conclude that the form of the animal had been moulded by its surroundings. Yet this first impression probably gives an entirely wrong conception of how such a relation has been acquired. Before we attempt to discuss this question, let us examine some typical examples.

A raclial type of structure is often found in fixed forms, and in some floating forms, like the jellyfish. In a fixed form, a sea-anemone, for instance, the conditions around the free end and the fixed end of the body are entirely different, and we find that these two ends are also different. The free end contains the special sense organs, the mouth, tentacles, etc.; while the fixed end contains the organ for attachment. It is eviclent that the free end is exposed to the same conditions in all directions, and it may seem probable that this will account for the radial symmetry of the anemone. There are also a few free forms, the sea-urchin for instance, that have a radial symmetry. Whether their ancestors were fixed forms, for which there is some evidence, we do not know definitely; but, even if this is true, it cloes not affect the main point, namely, that, although at present free to move, the sea-urchin is radially symmetrical. But when we 
examine its method of locomotion, we find that it moves indifferently in any direction over a solid surface; that is, it keeps its oral face against a solid object, and moves over the surface in any direction. Under these circumstances the same external conditions will act equally upon all sides of the body. In contrast to these common sea-urchins, there are two other related groups, in which, although traces of a well-marked radial symmetry are found, the external form has been so changed that a secondary bilateral form has been superimposed on it. These are the groups of the clypeasters and the spatangoids, and it is generally supposed that their forefathers were radially symmetrical forms like the ordinary forms of sea-urchins. These bilateral forms move in the direction of their plane of symmetry, but we have no means of knowing whether they first became bilateral and, in consequence, now move in the direction of the median plane, or whether they acquired the habit of moving in one direction, and in consequence acquired a bilateral symmetry. It seems more probable that the form changed first, for otherwise it is difficult to see why a change of movement in one direction should ever have taken place.

The radially symmetrical form is characteristic of many flowers that stand on the ends of their stalks. They also will be subjected to similar external influences in all directions. Many flowers, on the other hand, are bilaterally symmetrical. Some of these forms are of such a sort that they are generally interpreted as having been acquired in connection with the visits of insects. Be this as it may, it is still not clear why, if the flowers are terminal, insects should not approach them equally from every direction. If the flowers are not terminal, as, in fact, many of them are not, their relation to the surroundings is bilateral with respect to internal as well as to external conditions. The former, rather than the latter, may have produced the bilateral form of the flower. Here also we meet with the 

problem as to whether the flowers, being lateral in position, have assumed a bilateral form because their internal relations were bilateral; or whether an external relation, for example, the visits of insects, has been the principle cause of their becoming bilateral.
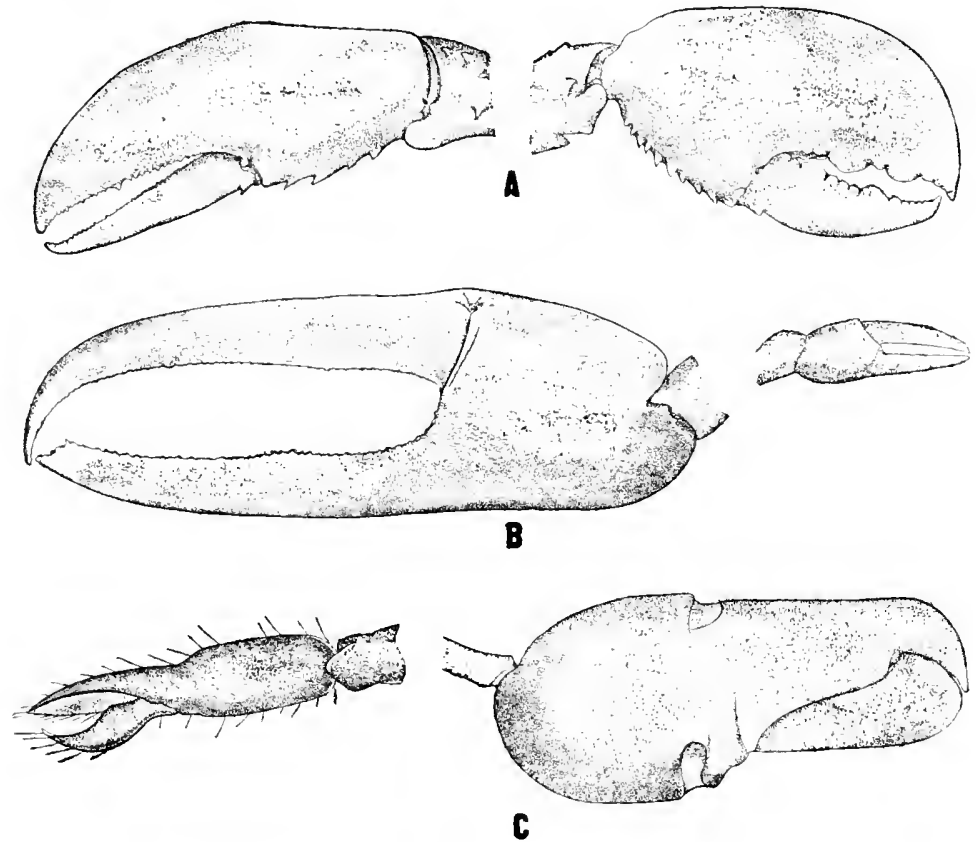

Fig. 4. - A, right and left claws of lobster: I', of the fiddler-crab; and C, of Alpheus.

In some bilateral forms the right and left sides may be unsymmetrical in certain organs. Right and left handedness in man is the most familiar example, although the structural difference on which this rests is not very obvious. More striking is the difference in the two big claws of the lobster (Fig. 4 A). One of the two claws is flat and has a fine sawtoothed edge. The other is thicker and has rounded knobs instead of teeth. It is said that these two claws are used by the lobster for different purposes, - the heavy one for crushing and for holding on, and the narrower for cutting up the 
food. If this is true, then we find a symmetrical organism becoming unsymmetrical, and in consequence it takes advantage of its asymmetry by using its right and left claws for different purposes.

More striking still is the difference in the size of the right and left claws in a related form, Alpheus - a crayfish-like form that lives in the sea. With the larger claw (Fig. 4 C) it makes a clicking sound that can be heard for a long distance. In some of the crabs the difference in the size of the two claws is enormous, as in the male fiddler-crab, for example (Fig. $4 \mathrm{~B}$ ). One of the claws is so big and umwieldy that it must put the animal at a distinct disadvantage. Its use is unknown, although it has been suggested that it is a secondary sexual character.

The asymmetry of the body of the snail is very conspicuous, at least so far as certain organs are concerned. The foot on which the animal crawls and the head have preserved their bilaterality; but the visceral mass of the animal, contained in the spirally wound shell, lying on the middle of the upper surface of the foot, is twisted into a spiral form. Many of the organs of one side of the body are atrophied. The gill, the kidney, the reproductive organ, and one of the auricles of the heart have completely, or almost completely, disappeared. The cause of this loss seems to be connected with the spiral twist of the visceral mass. One of the consequences of the twisting has been to bring the organs of the left side of the body around the posterior end until they come to lie on the right side, the organs of the original right side being carried forward and there atrophying.

There is another remarkable fact connected with the asymmetry of the snail. In some species, Helix pomatia, for example, the twist has been toward the right, i.e. in the direction which the hands of a watch follow when the face is turned upward toward the observer. Individuals twisted in this direction are called dextral. Occasionally there is 
found an individual with the spiral in the opposite direction (sinistral), and in this the conditions of the internal organs are exactly reversed. It is the left set of organs that is now atrophied, and the right set that is functional. Such changes appear suddenly. Organs of one side of the body that have not been functional for many generations may become fully developed. Moreover, Lang has shown that when a sinistral form breeds with a normal dextral form, or even when sinistral forms are bred with each other, the young are practically all of the ordinary type.

An attempt has been made to connect these facts with the mode of development of the mollusks. It is known that the eggs of a number of gasteropod mollusks segment in a perfectly definite manner. A sort of spiral cleavage is followed by the formation of a large mesodermal cell from the left posterior yolk-cell. From this mesodermal cell nearly all the mesodermal organs of the body are formed. Thus it may appear that the spiral form of the snail is connected with the spiral form of the cleavage. In a few species of marine and fresh-water snails the cleavage spiral is reversed, and the mesoderm arises from the right posterior yolk-cell. It has been shown in several cases that the snail coming from such an egg is twisted in the reverse direction from that of ordinary snails.

It has been suggested, therefore, that the occasional sinistral individual of Helix arises from an egg cleaving in the reverse direction, and there is nothing improbable in an assumption of this kind. No attempt has been made as yet to explain why, in some cases, the cleavage spiral is turned in one direction, and in other cases in the reverse direction; but even leaving this unaccounted for, the assumption of the unusual form of Helix being the result of a reversal of the cleavage throws some light as to how it is possible for the complete reversal of the organs of the adult to arise. If it is assumed that in the early embryo the cells on each side of 
the median line are alike, and at this time capable of forming adult structures, a simple change of the spiral from right to left might determine on which side of the middle line the mesodermal cell would lie, and its presence on one side rather than on the other might determine which sicle of the embryo would develop, and which would not. This possibility removes much of the mystery which may appear to surround a sudden change of this sort.

It seems to me that we shall not go far wrong if we assume that it is largely a matter of indifference whether an incliviclual snail is a right-handed or a left-handed form, as far as its relation to the environment is concerned. One form would have as good a chance for existing as the other. If this is granted, we may conclude that, while in most species a perfectly definite type is found, a right or a left spiral, yet neither the one nor the other has been acquired on account of its relation to the environment. This conclusion does not, of course, commit us in any way as to whether the spiral form of the visceral mass has been acquired in relation to the environment, but only to the view that, if a spiral form is to be produced, it is indifferent which way it turns. From the evolutionary point of view this conclusion is of some importance, since it inclicates that one of the alternatives has been adopted and has become practically constant in most cases without selection having had anything to do with it.

Somewhat similar conclitions are found in the flounclers and soles. As is well known, these fishes lie upon one side of the body on the bottom of the ocean. Some species, with the rarest exceptions to be mentioned in a moment, lic always on the right side, others on the left side. A few species are inclifferently right or left. At rare intervals a left-sided form is found in a right-sided species, and conversely, a right-sided form in a left-sicled species. In such cases the reversed type is as perfectly developed in all respects as the normal form, but with a complete reversal of its right and left sides. 
When the young flounders leave the egg, they swim in an upright position, as do ordinary fishes, with both sides equally developed. There cannot be any doubt that the ancestors of these fish were bilaterally symmetrical. Therefore, within the group, both right-handed and left-handed forms have appeared. It seems to me highly improbable that if a righthancled form had been slowly evolved through the selection of favorable variations in this direction, the end result could be suddenly reversed, and a perfect left-sided form appear. Moreover, as has been pointed out, the intermediate stages would have been at a great disadvantage as compared with the parent, and this would lead to their extermination on the selection theory. If, however, we suppose that a variation of this sort appeared at once, and was fixed, - a mutation in other words, - and that whether or not it had an advantage over the parent form, it could still continue to exist, and propagate its kind, then we avoid the chief difficulty of the selection theory. Moreover, we can imagine, at least, that if this variation appeared in the germ and was, in its essential nature, something like the relation seen in the snail, the occasional reversal of the relations of the parts presents no great difficulty.

In this same connection may be mentioned a curious fact first discovered by Przibram and later confirmed by others. If the leg carrying the large claw of a crustacean be removed, then, at the next moult, the leg of the other side that had been the smaller first leg becomes the new big one; and the new leg that has regenerated from the place where the big one was cut off becomes the smaller one.

Wilson has suggested that both claws in the young crustacean have the power to become either sort. We do not know what decides the matter in the adult, after the removal of one of the claws. Some slight difference may turn the balance one way or the other, so that the smaller claw grows into the larger one. At any rate, there is seen a latent power like 
that in the egg of the snail. Zeleny has found a similar reiation to exist for the big and the little opercula of the marine worm, Hydroides.

Let us consider now the more general questions involved in these symmetrical and asymmetrical relations between the organism and its environment. In what sense, it may be asked, is the symmetry of a form an adaptation to its environment? That the kind of symmetry gives to the animal in many cases a certain advantage in relation to its environment is so evident that I think it will not be questioned. The main question is how this relation is supposed to have been attained. Three points of view suggest themselves: First, that the form has resulted directly from the action of the environment upon the organism. This is the Lamarckian point of view, which we rejected as improbable. Second, that the form has been slowly acquired by selecting those individual variations that best suited it to a given set of surrounding conditions. This is the Darwinian view, which we also reject. The third, that the origin of the form has had nothing to do with the environment, but appeared independently of it. Having, however, appeared, it has been able to perpetuate itself under certain conditions.

It should be pointed out that the Darwinian view does not suppose that the environment actually produces any of the new variations which it selects after they have appeared, but in so far as the environment selects individual differences it is supposed to determine the direction in which evolution takes place. On the theory that evolution has taken place independently of selection, this latter is not supposed to be the case; the finished products, so to speak, are offered to the environment; and if they pass muster, even ever so badly, they may continue to propagate themselves.

The asymmetrical form of certain animals living in a symmetrical environment might be used as an argument to show that the relation of symmetry between an animal and 


\section{Origin of Different Kinds of Adaptations}

its environment can easily be overstepped without danger. The enormous claw of the fiddler-crab must throw the animal out of all symmetrical relation with its environment, and yet the species flourishes. The snail carries around a spiral hump that is entirely out of symmetrical relation with the surroundings of a snail.

These facts, few though they are, yet suffice to show, I believe, that the relation of symmetry between the organism and its environment may be, and is no doubt in many cases, more perfect than the requirements of the situation demand. The fact that animals made unsymmetrical through injuries (as when a crab loses several legs on one side, or a worm its head) can still remain in existence in their natural environment, is in favor of the view that I have just stated. By this I do not mean to maintain that a symmetrical form does not have, on the whole, an advantage over the same form rendered asymmetrical, but that this reiation need not have in all forms a selective value, and if not, then it cannot be the outcome of a process of natural selection.

To sum up: it appears probable that the laws determining the symmetry of a form are the outcome of internal factors, and are not the result either of the direct action of the environment, or of a selective process. The finished products and not the different imperfect stages in such a process, are what the inner organization offers to the environment. While the symmetry or asymmetry may be one of the numerous conditions which determine whether a form can persist or not, yet we find that the symmetrical relations may be in some cases more perfect than the environment actually demands; and in other cases, although the form may place the organism at a certain disadvantage, it may still be able to exist in certain localities. 


\section{Mutual Adaptation of Colonial Forms}

In the white ants, true ants, and bees, we find certain individuals of the community specialized in such a way that their modifications stand in certain useful relations to other members of the community. Amongst the bees, the workers collect the food, make the comb, and look after the young. The queen does little more than lay eggs, and the drone's only function is to fertilize the queen. In the true ants there are, besides the workers and the queen and the males, the soldier caste. These have large thick heads and large strong jaws. On the Darwinian theory it is assumed that this caste must have an important rôle to play, for otherwise their presence as a distinct group of forms cannot be accounted for ; but I clo not believe it is necessary to find an excuse for their existence in their supposed utility. From the point of view of the mutation theory, their real value may be very small, but so long as their actual presence is not entirely fatal to the community they may be endured.

In regard to these forms, Sharp writes: " "The soldiers are not alike in any two species of Termitidx, so far as we know, and it seems impossible to ascribe the differences that exist between the soldiers of different species of Termitida to special adaptations for the work they have to perform." "On the whole, it would be more correct to say that the soldiers are very dissimilar in spite of their having to perform similar work, than to state that they are dissimilar in conformity with the different tasks they carry on." The soldiers have the same instincts as the workers, and do the same kinds of things to a certain extent. "The soldiers are not such effective combatants as the workers are." Statements such as these indicate very strongly that the origin of this caste can have very little to do with its importance as a specialized part of the community. 
The differences between the castes have gone so far in some of these groups that the majority of the members of the community have even lost the power to reproduce their kind, and this function has devolved upon the queen, whose sole duty is to reproduce the different castes of which the community is composed. This specialization carries with it the idea of the individuals being adapted to each other, so that, taken all together, they form a whole, capable of maintaining and reproducing itself. It does not seem that we must necessarily look upon this union as the result of competition leading to a death struggle between different colonies, so that only those have survived in each generation that carried the work of specialization one step farther. All that is required is to suppose that such specialization has appeared in a group of forms living together, and the group has been able to perpetuate itself. We do not find that all other members of the two great groups to which the white ants and true ants belong have been crowded out because these colonial forms have been evolved. Neither need we suppose that during the evolution of these colonial species there has been a death struggle accompanying each stage in the evolution. If the members of a colonial group began to give rise to different forms through mutations, and if it happened that some of the combinations formed in this way were capable of living together, and perpetuating the group, this is all that is required for such a condition to persist.

The relation of the parents to the offspring presents in some groups a somewhat parallel case to that of these colonial forms. Not only are some of the fundamental instincts of the parents changed, but structures may be present in the parents whose only use is in connection with the young. The marsupial pouch of the kangaroo, in which the immature young are carried and suckled, is a case in point, and the mammary glands of the Mammalia furnish another illustration. Adaptations of these kinds are clearly connected with the 
perpetuation of the race. In the case of the mammals the young are born so immature that they are dependent on the parental organs, just spoken of, for their existence. Could we follow this relation through its evolutionary stages, it would no doubt furnish us with important data, but unfortunately we can do no more than guess how this relation became established. The changes in the young and in the parent may have been intimately connected at each stage, or more or less independent. If we suppose the mammary glands to have appeared first, they might have been utilized by the young in order to procure food. Their presence would then make it possible for the young to be born in an immature condition, as is the case with the young of many of the mammals. But this is pure guessing, and until we know more of the actual process of evolution in this case, it is unprofitable to speculate.

\section{Degeneration}

In almost every group of the animal kingdom there are forms that are recognized as degenerate. This degeneration is usually associated with the habitat of the animal. In many cases it can be shown with much probability that these degenerate forms have descended from members of the group that are not degenerate. We find there is a loss of those organs that are not useful to the organism in its new environment. The degeneration may involve nearly the whole organization (except as a rule the reproductive system), as seen in the tapeworm, or only certain organs of the body, as the eyes in cave animals. A few examples will bring the main facts before us.

A parasitic existence is nearly always associated with degeneration. Uncler these conditions, food can generally be obtained without difficulty, at the expense of the host, and apparently associated with this there is a degeneration, and 
even a complete loss of so important an organ as the digestive tract. Thus the tapeworm has lost all traces of its digestive tract, absorbing the already digested matter of its host through its body wall. Some of the roundworms, that live in the alimentary tracts of other animals, may have their digestive organs reduced. I $\mathrm{I}$ Trichina, this degeneration has gone so far that the digestive tract is represented, in part, by a single line of endoderm cells, pierced by a cavity. The digestive organs are also absent in certain male rotifers, which are parasitic on the females, and these organs are also very degenerate in the male of Bonellia, a gephyrean worm. A parasitic snail, Entoscolar ludwigii, has its digestive apparatus reduced to a sucking tube ending in a blind sac. The rest of the tract has completely degenerated. The remarkable parasitic crustacean, Sacculina carcini, looks like a tumor attached to the under surface of the abdomen of a crab. It has neither mouth nor digestive tract, and absorbs nourishment from the crab through rootlike outgrowths that penetrate the body. From its development alone we know that it is a degenerate barnacle.

There seems to be in all these cases an apparent connection between the absence of the cligestive tract and the presence of an abundant supply of food, that has already been partly digested by the host. Put in a clifferent way, we may say that the presence of this food has furnished the environment in which an animal may live that has a rudimentary digestive tract.

An interesting case of degeneration is found in the rudimentary mouth parts of the insects known as May-flies, or ephemerids. Some of these species live in the adult condition for only a few hours, only long enough to unite and deposit their eggs. In the adult stage the insects do not take any food. In this case the degeneration is obviously not connected with the presence of food, but apparently with the shortness of the adult life. 
One of the most familiar cases of degeneration is blind. ness, associated with life in the dark. The most striking cases are those of cave animals, but this is only an extreme example of what is found everywhere amongst animals that live concealed during the day under stones, ete. The blind fish and the blind crayfish of the Mammoth Cave, the blind proteus of the caves of Carniola, the blind mole that burrows underground, the blind larva of many insects that live in the dark, are examples most often cited. Some nocturnal animals, like the earthworm, have no eyes, although they are still able to distinguish light; and some of the deepsea animals, that live below the depth to which light penetrates, have degenerate eyes. The workers of some ants, that remain in the nests, are blind, but the males and the queens of these forms have well-developed cyes, although the eyes may be of use to them at only one short period of their life, namely, at the time of the marriage flight. This fact is significant and is underestimated by those who believe that disuse accounts for the degeneration of organs.

The wings of the ostrich and of the kiwi are rudimentary structures no longer used for flight, and many insects, belonging to several different orders, have lost their wings, as seen in fleas, some kinds of bugs, and moths, and even in some grasshoppers.

A curious case of degeneration is found in the abdomen of the hermit crab, which is protected by the appropriated shell of a snail. The appendages of one side of the abdomen have nearly disappeared in the male, although in the female the abdominal appendages are used to carry the eggs as in other decapod crustaceans. The abdomen, instead of being covered by a hard euticle, as in other members of this group, is soft and unprotected except by the shell of the snail.

Cases of these kinds could be added to almost indefinitely, and the explanation of these degenerate structures has been a source of contention amongst zoologists for a long time. 
The most obvious interpretation is that the degeneration has been the result of disuse. But as I have already discussed this question, and given my reasons for regarding it as improbable that degeneration has arisen in this way, we need not further consider this point here.

The selectionists have offered several suggestions to account for degeneration. In fact, this has been one of the difficulties that has given them most concern. They have suggested, for example, that when an organ is no longer of use to its possessor it would become a source of danger, and hence would be removed through natural selection. They have also suggested that since such organs draw on the general food supply they would place their possessor at a disadvantage, and hence would be removed. Weismann has attempted to meet the difficulty by his theory of "Panmixia," or universal crossing, by which means the useless structures are imagined to be eliminated.

These attempts will suffice to point out the straits to which the Darwinians have found themselves reduced, and we have by no means exhausted the list of suggestions that have been made. Let us see, if, on any other view, we can avoid some of the difficulties that the selection theory has encountered.

In the first place we shall be justified, I think, in eliminating competition as a factor in the process, since the admission that an organ has become useless carries with it the idea that it has no longer a selective value. If, in its useless condition, it is no longer greatly injurious, as is probably, though not necessarily always, the case, then selection cannot enter into the problem. If in parasitism we assume that an animal finds a lodgement in another animal, where it is able to exist, we may have the first stage of the process introduced at once. If under these conditions a mutation appeared, involving some of the organs that are no longer essential to the life of the individual in its new environment, 
the new mutation may persist. We need not suppose that the original form becomes crowded out, but only that a more degenerate form has come into existence. As a matter of fact we find in most groups, in which degenerate forms exist, a number of different stages in the degeneration in different species. Mutation after mutation might follow until many of the original organs have disappeared. The connection that appears to exist between the degeneration of a special part and the environment in which the animal lives finds its explanation simply in the fact that the environment makes possible the existence of that sort of mutation in it. IVe do not know, as yet, whether through mutative changes an organ can completely disappear, although this seems probable from the fact that in a few cases mutations are known to have arisen in which a given part is entirely functionless. If we could assume that, a mutation in the direction of degeneration being once established, further mutations in the same direction would probably occur, the problem would be much simplified; but we lack data, at present, to establish this view.

In the case of blind animals it seems probable that the transition has taken place in such forms as had already established themselves in places more or less removed from the light. Such forms as had the habit of hiding away under stones, or in the ground, living partly in and partly out of the light, might, if a mutation appeared of such a sort that amongst other changes the eyes were less developed, still be capable of leading an existence in the dark, while it might be impossible for them to exist any longer with weakened vision in the light. If such a process took place, the habitat of the new form would be limited, or in other words it would be confined to the locality to which it finds itself adapted; not that it has become adapted to the environment through competition with the original species, or, in fact, with any other. 
Thus, from the point of view that is here taken, an animal does not become degenerate because it becomes parasitic, but the environment being given, some forms have found their way there; in fact, we may almost say, have been forced there, for these degenerate forms can only exist under such conditions.

In conclusion, this much at least can be claimed for the mutation theory; that it meets with no serious difficulty in connection with the phenomena of degeneration. It meets with no difficulty, because it makes no pretence to explain the origin of adlaptations, but can account for the occurrence of degenerate forms, if it is admitted that these appear as mutations, or as definite variations. Let us, however, not close our eyes to the fact that there is still much to be explained in respect to the degeneration of animals and plants. It is far from my purpose to apply the mutation theory to all adaptations; in fact, it will not be clifficult to show that there are many adaptations whose existence can have nothing directly to do with the mutation theory.

\section{Protective Coloration}

That many species of animals are protected by their resemblance to their environment no one will probably deny. That we are ignorant in all cases as to how far this protection is necessary for the maintenance of the species must be admitted. That some of the resemblances that have been pointed out have been given fictitious value, I believe very probable.

Resemblance in color between the organism and its environment has given to the modern selectionist some of his most valuable arguments, but we should be on our guard against supposing that, because an animal may be protected by its color, the color has been acquired on this account. On the supposition that the animal has become adapted by 
degrees, and through selection, we meet with all the objections that have been urged, in general, against the theory of natural selection. But if we assume here also that mutations have occurred without relation to the environment, and, having once appeared, determined in some cases the distribution of the species, we have at least a simple hypothesis that appears to explain the facts. If it be claimed that the resemblance is, in some cases, too close for us to suppose that it has arisen independently of the environment, it may be pointed out that it has not been shown that such a close resemblance is at all necessary for the continued existence of the species, and hence the argument is likely to prove too much. For instance, the most remarkable case of resemblance is that of Kallima, but in the light of a recent statement by Dean it may be seriously asked whether there is absolute need of such a close resemblance to a leaf. Even if it be admitted that to a certain extent the butterfly is at times protected by its resemblance to a leaf, it is not improbable that it could exist almost equally well without such a close resemblance. If this is true, natural selection could never have brought about such a close imitation of a leaf. Cases like these of over-adaptation are not unaccountable on the theory of mutation, for on this view the adaptation may be far ahead of what the actual requirements for protection demand. We meet occasionally, I think, throughout the living world with resemblances that can have no such interpretation, and a number of the kinds of adaptations to be described in this chapter show the same relation.

Some of the cases of mimicry appear also to fall under this head; although I do not doubt that many so-called cases of mimicry are purely imaginary, in the sense that the resemblance has not been acquired on account of its relation to the animal imitated. There is no need to question that in some cases animals may be protected by their resemblance to other animals, but it does not follow, despite the vigorous 
assertions of some modern Darwinians, that this imitation has been the result of selection. Until it can be shown that the imitating species is dependent on its close imitation for its existence, the evidence is unconvincing; and even if, in some cases, this should prove to be the case, it does not follow that natural selection has brought about the result, or even that it is the most plausible explanation that we have to account for the results. The mutation theory gives, in such cases, an equally good explanation, and at the same time avoids some of the clifficulties that appear fatal to the selection theory.

What has been said against the theory of mimicry might be repeated in much stronger terms against the hypothesis of warning colors.

It seems to me, in this connection, that the imagination of the selectionist has sometimes been allowed to "run wild"; and while it may be true that in some cases the colors may serve as a signal to the possible enemies of the animal, it seems strange that it has been thought necessary to explain the origin of such colors as the result of natural selection. Indeed, some of these warning colors appear unnecessarily conspicuous for the purpose they have to perform. In other words, it does not seem plausible that an animal already protected should need to be so conspicuous. If we stop for a moment to consider what an enormous amount of destruction must have occurred, according to Darwin's theory, in order to bring this warning coloration to its supposed state of perfection, we may well hesitate before committing ourselves to such an extreme view.

That gaudy colors have appeared or been present in animals that are protected in other ways is not improbable, when we consider the rôle that color plays everywhere in nature. That the presence of such colors may, to a certain limited extent, protect its possessor may be admitted without in any degree supposing that natural selection has directed 
the evolution of such color, or that it has been acquired through a life and death struggle of the individuals of the species.

\section{Sexual Dimorphism and Trimorphism}

It has been found in a few species of animals and plants that two or more forms of one sex may exist, and here we find a condition that appears to be far more readily explained on the mutation theory than on any other. The most important cases, perhaps, are those in plants, but there are also similar cases known amongst animals, and these will be given first.

There is a North American butterfly, Papilio turnus, that appears under at least two forms. In the eastern United States the male has yellow wings with black stripes. There are two kinds of females, one of which resembles the male except that she has also an orange "eye-spot"; the other female is much blacker, and this variety is found particularly in the south and west. The species is dimorphic, therefore, mainly in the latter regions.

The cases of seasonal dimorphism offer somewhat similar illustrations. The European butterfly, I'ancssa learana-prorsa, has a spring generation (lacancy) with a yellow and black pattern on the upper surface of the wings. The summer generation (prorsa) has black wings "with a broad white transverse band, and delicate yellow lines running parallel to the margins." These two types are sharply separated, and their differences in color do not appear to be associated with any special protection that it confers on the bearer. These facts in regard to Vanessa seem to indicate that differences may arise that are perfectly well marked and sharply defined, which yet appear to be without any useful significance.

1 This term is used here in the sense employed by Darwin. The same term is sometimes used for those cases in which the male departs very greatly from the female in form. 


\section{Origin of Different Kinds of Adaptations $36 \mathrm{I}$}

We meet with cases in which the same animal has at different times of year clifferent colors, as seen in the summer and winter plumage of the ptarmigan. There is no direct evidence to show how this seasonable change has been brought about; but from the facts in regard to Vanessa we can see that it might have been at least possible for the white winter plumage, for instance, to have appeared without respect to any advantage it conferred on the animal, but after it had appeared it may have been to a certain degree useful to its possessor.

Amongst plants there are some very interesting cases of dimorphism and trimorphism in the structure of the flowers. Darwin has studied some of these cases with great care, and has made out some important points in regard to their powers of cross-fertilization. ${ }^{1}$ The common European cowslip, Primula weris, var. officinalis, is found under two forms, Figure 5 $A$ and $B$, which are about equally abundant. In one the style is long so that the stigma borne on its end comes to the top of the tube of the corolla. The stamens in this form stand about halfway up the tube. This is called the longstyled form. The other kind, known as the short-styled form, has a style only half as long as the tube of the corolla, and the stamens are attached around the upper end of the tube near its opening. In other words, the position of the end of the style (the stigma) and that of the stamens is exactly reversed in the two forms. The corolla is also somewhat differently shaped in the two forms, and the expanded part of the tube above the stamens is larger in the long-styled than in the short-styled form. Another clifference is found in the stigma, which is globular in the long-styled, and depressed on its top in the short-styled, form. The papillæ

1 Many of the facts as to the occurrence of these cases were known before Darwin worked on them; but very little had been ascertained in regard to the sexual relation between the dimorphic and trimorphic forms, and it was here that Darwin obtained his most interesting results. 
on the former are twice as long as those on the short-styled form. The most important difference is found in the size of the pollen grains. These are larger in the long-styled form, being in the two cases in the proportion of 100 to 67 . The shape of the grains is also different. Furthermore, the long-

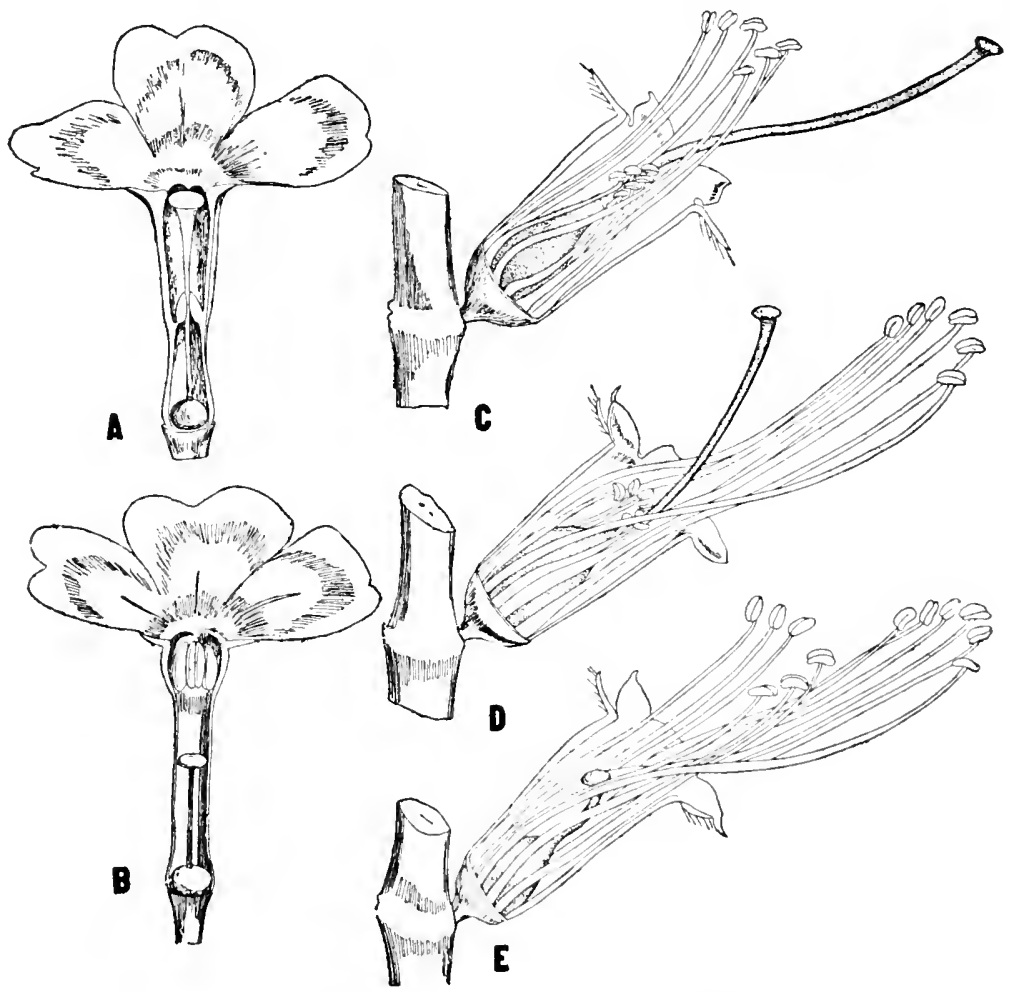

FIG. 5. - A, long-styled, and B, short-styled, forms of Primula arris. C, D, E, the three forms of the trimorphic flower of Lythrum saliaria, with petals and calyx removed on near side. (After Darwin.)

styled form tends to flower before the other kind, but the short-styled form produces more seeds. The orules in the long-styled form, even when unfertilized, are considerably larger than those of the short-styled, and this, Darwin suggests, may be connected with the fact that fewer seeds are produced, since there is less room for them. The important 


\section{Origin of Different Kinds of Adaptations $3_{3}{ }_{3}$}

point for our present consideration is that intermediate forms do not exist, although there are fluctuating variations about the two types. Moreover, the two kinds of flowers never appear on the same plant.

Darwin tried the effect of fertilizing the long-styled flowers with the pollen from the same flower or from other longstyled flowers. Unions of this sort he calls illegitimate, for reasons that will appear later. He also fertilized the longstyled flowers with pollen from short-styled forms. A union of this sort is called legitimate. Conversely, the short-styled forms were fertilized with their own pollen or with that from another short-styled form. This is also an illegitimate union. Short-styled forms fertilized with pollen from long-styled forms give again legitimate unions.

The outcome of these different crossings are most curious. In the table, page 364 , the results of the four combinations are given. It will be seen at once that the legitimate unions give more capsules, and the seeds weigh more, than in the illegitimate unions.

The behavior of the offspring from seeds of legitimate and illegitimate origin is even more astonishing. Darwin found in Primula veris (the form just described) that the seeds from the short-styled form fertilized with pollen from the same form germinated so badly that he obtained only I 4 plants, of which 9 were short-styled and 5 long-styled. The long-styled form fertilized with its own-styled pollen produced "in the first generation 3 long-styled plants. From their seed 53 long-styled grandchildren were produced; from their seed 4 long-styled great-grandchildren; from their seed 20 long-styled greatgreat-grandchildren; and lastly, from their seed 8 long-styled and 2 short-styled great-great-great-g randchildren."

From other long-styled plants, fertilized with their ownform pollen, 72 plants were raised, which were made up of 68 long-styled and 4 short-styled. In all, I62 illegitimate unions of this sort produced I 56 long-styled and 6 short- 


\begin{tabular}{|c|c|c|c|c|c|}
\hline Nature of Union & $\begin{array}{l}\text { NUMBER } \\
\text { OF Flowers } \\
\text { FERTLLIZEd }\end{array}$ & $\begin{array}{l}\text { Number } \\
\text { of Seed } \\
\text { Capsules }\end{array}$ & $\begin{array}{l}\text { MaxiMlM OF } \\
\text { SEEDSIN ANY } \\
\text { ONECAPSULE }\end{array}$ & $\begin{array}{l}\text { MINIMUM OF } \\
\text { SEEDS INANY } \\
\text { ONE CAPSULE }\end{array}$ & $\begin{array}{c}\text { Average } \\
\text { No. OF SEeds } \\
\text { PER Capsule }\end{array}$ \\
\hline $\begin{array}{l}\text { Long-styled form by } \\
\text { pollen of short- } \\
\text { styled form: } \\
\text { Legritimate union. }\end{array}$ & 10 & 6 & 62 & 34 & 46.5 \\
\hline $\begin{array}{l}\text { Long-styled form by } \\
\text { own-form pollen: } \\
\text { Illegitimate union. }\end{array}$ & 20 & 4 & 49 & 2 & $27 \cdot 7$ \\
\hline $\begin{array}{l}\text { Short-styled form by } \\
\text { pollen of long- } \\
\text { styled form : } \\
\text { Legitimate union. }\end{array}$ & IO & 8 & $6 I$ & 37 & $47 \cdot 7$ \\
\hline $\begin{array}{c}\text { Short-styled form by } \\
\text { own-form pollen : } \\
\text { lllegitinate union. }\end{array}$ & I 7 & 3 & 19 & 6 & 12.1 \\
\hline $\begin{array}{c}\text { The two legitimate } \\
\text { unions together. }\end{array}$ & 20 & I4 & 62 & 37 & $47 \cdot 1$ \\
\hline $\begin{array}{c}\text { The two illegitimate } \\
\text { unions together. }\end{array}$ & 30 & 7 & 49 & 2 & $35 \cdot 5$ \\
\hline
\end{tabular}

styled plants. It is evident from these results that the longform pistils, fertilized with pollen from flowers of the same pistil-form (from other individuals as a rule), tend to produce the same form as their parents, although occasionally the other form. The fertility of these plants from an illegitimate union is found to be very low. Darwin observed that sometimes the male and female organs of these plants were in a very deteriorated condition. It is interesting to notice, in this connection, that in another species, Primula sincnsis, illegitimate plants from long-styled parents were vigorous, but the flowers were small and more like the wild form. They were, however, perfectly fertile. 


\section{Origin of Different Kinds of Adaptations 365}

Illegitimate plants from short-styled parents were dwarfed in stature, and often had a weakly constitution. They were not very fertile inter se, and remarkably infertile when legitimately fertilized. This kind of result, where a difference in the power of mutual intercrossing exists between two forms, recalls in many ways the difference in the results of crossing of different species of animals and plants, especially those cases in which a cross can be made in one way more successfully than in the other.

The heterostyled trimorphic plants, of which Lythrum salicaria, Figure $5 \mathrm{C}, \mathrm{D}, \mathrm{E}$, may be taken as an example, are even more remarkable. There are three different kinds of flowers: in one the pistil is long and there is a medium and a short set of stamens; in another the pistil is of intermediate length and there is a long set and a short set of stamens; in the third kind the pistil is short, and there is a medium and a long set of stamens. There are possible only six sorts of legitimate unions between these three sets of flowers. No less than twelve kinds of illegitimate unions may occur. In regard to the difference in the sizes of the pollen grains, those from the long-styled form are the largest, from the mid-styled form next, and from the short-styled form the smallest. The extreme difference is as I00 to 60 . "Nothing shows more clearly the extraordinary complexity of the reproductive system of this plant than the necessity of making eighteen distinct unions in order to ascertain the relative fertilizing power of the three forms." Darwin tried the effect of each of these combinations, making 223 unions in all. The results are surprising. Comparing the outcome of the six legitimate unions with the twelve illegitimate ones. the following results were obtained :- 


\begin{tabular}{|c|c|c|c|c|}
\hline NATURE OF UNION & $\begin{array}{l}\text { NUMber } \\
\text { OF Flowers } \\
\text { FERTiLized }\end{array}$ & $\begin{array}{c}\text { Number } \\
\text { of Capslles } \\
\text { Prodiced }\end{array}$ & $\begin{array}{c}\text { Average } \\
\text { No. of SeEds } \\
\text { PER Capsule }\end{array}$ & $\begin{array}{c}\text { Alerage } \\
\text { No. of SeEds } \\
\text { PER Flower } \\
\text { Fertilized }\end{array}$ \\
\hline The 6 legitimate unions & 75 & 56 & 96.29 & 71.89 \\
\hline The 12 illegitimate unions & 146 & 36 & $44 \cdot 72$ & I I .03 \\
\hline
\end{tabular}

This table shows that the fertility of the legitimate to that of the illegitimate is as 100 to 33 , as judged by the flowers that produced capsules; and as 100 to 46 as judged by the average number of seeds per capsule. It is evident, therefore, that "it is only the pollen from the longest stamens that can fully fertilize the longest pistil; only that from the mid-length stamens, the mid-length pistil; and only that from the shortest stamens, the shortest pistil."

Darivin tries to connect this fact with the visits of insects to the flowers. He says: "And now we can comprehend the meaning of the almost exact correspondence in length between the pistil in each form and of a set of six stamens in two of the other forms; for the stigma of each form is thus rubbed against that part of the insect's body which becomes charged with the proper pollen." A further conclusion that Darwin draws is "that the greater the inequality in length between the pistil and the set of stamens, the pollen of which is employed for its fertilization, by so much is the sterility the more increased." Darwin also makes the following significant comment on the problem here involved: "The correspondence in length between the pistil in each form, and a set of stamens in the other two forms, is probably the direct result of adaptation, as it is of the highest service to the species by leading to full and legitimate fertilization." He points out, on the other hand, that the increased sterility of the illegitimate unions, in proportion to the inequality in length between the pistil and the stamens 
employed, can be of no service at all. Neither can this relation have any connection with the facility for self-fertilization. "We are led, therefore, to conclude that the rule of increased sterility in accordance with increased inequality in length between the pistils and stamens is a purposeless result, incidental on those changes through which the species has passed in acquiring certain characters fitted to insure the legitimate fertilization of the three flowers."

In regard to the plants that were raised from the seeds from legitimate and illegitimate unions, Darwin found in Lythrum that of twelve illegitimate unions two were completely barren, and nearly all showed lessened fertility; only one approached complete fertility. Darwin lays much emphasis on the close resemblance in the sterility of the illegitimate unions, and the sterility of different species when crossed. In both cases every degree of sterility is met with, "from very slightly lessened fertility to absolute barrenness." The importance of this comparison cannot, I think, be overestimated, for, if admitted, it indicates clearly that the infertility between species cannot be used as a criterion of their distinctness, because here, in individuals belonging to the same species, we find sterility between pistils and stamens of clifferent lengths. If, as I shall urge below, we must consider these different forms of Primula the results of a mutation, and not the outcome of selection as Darwin supposed, then this relation in regard to infertility becomes a point of great interest.

This brings us to the central point of our examination of these cases of dimorphism and trimorphism. How have these forms arisen? Darwin tries to account for them as follows: Since heterostyled plants occur in fourteen different families of plants, it is probable that this condition has been acquired independently in each family, and "that it can be acquired without any great difficulty." The first step in the process he imagines to have been due to great variability 
in the length of the pistil and stamens, or of the pistil alone. Flowers in which there is a great deal of variation of this sort are known. "As most plants are occasionally crossfertilized by the aid of insects, we may assume that this was the case with our supposed varying plant; but that it would have been beneficial to it to have been more regularly crossfertilized." "This would have been better accomplished if the stigma and the stamens stood at the same level; but as the stamens and pistil are supposed to have varied much in length, and to be still varying, it might well happen that they could be reduced much more easily through natural selection into two sets of different lengths in different individuals than all to the same length and level in all individuals." By means of these assumptions, improbable as they may appear, Darwin tries to explain these cases of dimorphism. But when we attempt to apply the same argument to the trimorphic forms, it is manifestly absurd to pretend that three such sharply defined types could ever have been formed as the result of natural selection. But we have not even yet touched the chief difficulty, as Darwin himself points out. "The essential character of a heterostyled plant is that an individual of one form cannot fully fertilize, or be fertilized by, an individual of the same form, but only by one belonging to another form." This result Darwin admits cannot be explained by the selection theory, for, as he says, "How can it be any advantage to a plant to be sterile with half of its brethren, that is, with the individuals belonging to the same form?" He concludes that this sterility between the individuals of the same form is an incidental and purposeless result. "Inner constitutional differences" between the individuals is the only suggestion that is offered to account for the phenomenon. In other words, it is clearly apparent that the attempt to apply the theory of selection has here broken down, and it is a fortunate circumstance that the Lamarckian theory cannot here be brought to the rescue, as 
it so often is in Darwin's writings, when the theory of natural selection fails to give a sufficient explanation.

On the other hand, this is one of the cases that seem to fit in excellently with the mutation theory, for if these two forms of the primrose should appear, as mutations, and if, as is the case, they do not blend when crossed, but are equally inherited, they would both continue to exist as we find them to-day. Whether the similar forms were infertile with each other would be determined at the outset by the nature of the individual variation, and if, despite this obvious disadvantage, the forms could still continue to propagate themselves, the new dimorphic form would remain in existence. Darwin cannot explain the origin of climorphic forms and trimorphic forms unless he can show that there is some advantage in having two forms, and as we have seen, he fails completely to show that there is an advantage. On the other hand, the result might have been reached on the mutation theory, even if the dimorphic and trimorphic forms were placed at a greater disadvantage than were the parent forms. In such a case fewer individuals might appear, or find a foothold; but as long as the race could be kept up the new forms would remain in existence. Thus, while no attempt is made to explain what has always been, and may possibly long remain, inexplicable to us, namely, the origin of the new form itself, yet granting that such new forms may sometimes appear spontaneously, they may be able to establish themselves, regardless of whether they are a little more or a little less well adapted to the environment than were their parent forms. If it should appear that the question is begged by the assumption that mutations such as these may appear (at one step or by a series of steps is immaterial), it should not be forgotten that the whole Darwinian theory itself also rests on the spontaneous appearance of fluctuating variations, whose origin it does not pretend to explain. In this respect both theories are on the same footing, but where the 
Darwinian theory meets with difficulties at every turn by assuming that new forms are built up through the action of selection, the mutation theory escapes most of these difficulties, because it applies no such rigid test as that of selection to account for the presence of new forms.

\section{Length of Life as an Adaptation}

It has been pointed out in the first chapter that the length of life of the individual has been supposed by some of the most enthusiastic followers of Darwin to be determined by the relation of the individual to the species as a whole. In other words, the doctrine of utility has been applied here also, on the ground that it would be detrimental to the species to have part of the individuals live on to a time when they can no longer propagate the race or protect the young. It is assumed that those varieties or groups of individuals (unfortunately not sharply defined) would have the best chance to survive in which the parent forms died as soon as they had lost the power to produce new individuals. Sometimes interwoven with this idea there is another, namely, that dcath itsclf has been acquired because it was more profitable to supplant the old and the injured individuals by new ones, than to have the old forms survive, and thus deprive the reproducing individuals of some of the common food supply.

This insidious form that the selection theory has taken in the hands of its would-be advocates only serves to show to what extremes its disciples are willing to push it. On the whole it would be folly to pursue such a will-o'-the-wisp, when the theory can be examined in much more tangible cxamples. If in these cases it can be shown to be improbable, the remaining superstructure of quasi-mystical hypothesis will fall without more ado.

That the problem of the length of life may be a real one for physiological investigation will be granted, no cloubt, with- 
out discussion, and that in some cases the length of life and the coming to maturity of the germ-cell may be, in some way, physiologically connected seems not improbable; but that this relation has been regulated by the competition of species with each other can scarcely be seriously maintained. I will not pretend to say whether the mutation theory can or cannot be made to appear to give the semblance of an explanation of the length of life in each species, but it seems to me fairly certain that this is one of the questions which we are not yet in a position to attempt to consider on any theory of evolution.

\section{Organs of Extreme Perfection}

It has often been pointed out that certain organs may be more perfectly developed than the requirements of the surroundings strictly demand. At least we have no good reasons to suppose in some cases that constant selection is keeping certain organs at the highest possible point of development, yet, on the Darwinian theory, as soon as selection ceases to be operative the level of perfection must sink to that which the exigencies of the situation demand. The problem may be expressed in a different way. Does the animal or plant ever possess organs that are more perfectly adapted than the absolute requirements demand? If such organs are the result of fluctuating variations, they will be unable to maintain themselves in subsequent generations without a constant process of selection going on. If, on the other hand, the organs have arisen as mutations, they may become permanently established without respect to the degree of perfection of their adaptation. We can see, therefore, that cases of extreme perfection meet with no difficulty on the mutation theory, while they have proven one of the stumbling-blocks to the selection theory.

There are, in fact, many structures in the animal and plant kingdoms that appear to be more perfect than the require- 
ments seem to demand. The exact symmetry of many forms appears in some cases to be unnecessarily perfect. The perfection of the hand of man, the development of his vocal organs, and certain qualities of his brain, as his musical and mathematical powers, seem to go beyond the required limits. It is not, of course, that these things may not be of some use, but that their development appears to have gone beyond what selection requires of these parts.

Closely related to this group of phenomena are those cases in which certain organs are well developed, but which can scarcely be of use to the animal in proportion to their elaboration. The electric organs of several fishes and skates are excellent examples of this sort of structures. The phosphorescent organs do not appear, in some forms at least, to be useful in proportion to their development. The selection theory fails completely to explain the building up of organs of this kind, but on the mutation theory there is no difficulty at all in accounting for the presence of even highly developed organs that are of little or of no use to the individual. If the organs appeared in the first place as mutations, and their presence was not injurious to the extent of interfering seriously with the existence and propagation of the new form, this new form may remain in existence, and if the mutations continued in the same direction, the organs might become more perfect, and highly developed. The whole class of secondary sexual organs may belong to this category, but a discussion of these organs will be deferred to the following section.

\section{Secondary Sexual Organs as Adaptations}

In the sixth chapter we have examined at some length Darwin's interpretation of the secondary sexual characters. I Is explanation has been found insufficient in many cases to account for the conditions. That these organs clo play in some cases a rôle in the relation of the sexes to each other may be freely admitted. In other words, in some animals the 
organs in one sex appear in the light of adaptations to certain instincts in the other sex. It would, perhaps, appear to simplify the problem to deny outright that any such relation exists ; but I think, in the light of the evidence that we have, this procedure would be like that of the proverbial ostrich, which is supposed to stick its head in the sand in order to escape an anticipated danger. If we assumed this agnostic position, we might attempt to account for the appearance of secondary sexual organs as mutations that had appeared in one sex, and had no immediate connection with the other sex; and, so long as these organs were not directly and seriously injurious, we might assume that the animals in which such structures had appeared might be able to exist. But, on the other hand, I think that an examination of the evidence will show that this way out of the difficulty is not very satisfactory, for the organs in question appear, in some cases at least, to be closely connected with certain definite responses in the other sex. Moreover, as Darwin has so insistently pointed out, the action of the males is of such a sort that it is eviclently associated with the presence of the secondary sexual organs which they often display before the other sex. Furthermore, the greater and often exclusive development of these organs during the sexual period distinctly points to them as in some way connected with the relation of the sexes to each other. And finally, there is a small, although not entirely convincing, body of evidence, indicating that the female is infuenced by the action of the male; but I do not think that this evidence shows that she selects one individual at the expense of all other rivals: We meet here with a problem that is as profoundly interesting as it is obscure. In fact, if we admit that this relation exists we have a double set of conditions to deal with: first, the development in the males of certain secondary sexual organs; and secondly, the instinct to display these organs. The supposed influence of the display on 
the female may also have to be taken into account, although, for all we know to the contrary, the same results might follow were there no secondary sexual character at all, as is, in fact, the case in most animals.

I have a strong suspicion that much that has been written on this subject is imaginative, and in large part fictitious; so that it may, after all, be the wisest course not to attempt to explain how this relation has arisen until we have a more definite conception of what we are really called upon to explain. For example, when we see a gorgeously bedecked male displaying himself before a female, we feel that his finery must have been acquired for this very purpose. On the other hand, when we see an unornamented male also making definite movements before the female, we do not feel called upon to explain the origin of his colors. Now, it is not improbable that the ornaments of the first individual have not been acquired in order to display them before the female, and this view seems to me the more probable. From this standpoint our problem is at least much simplified. What we need to account for is only that the male is excited to undergo certain movements in the presence of the female, and possibly that the female may be influenced by the result. That this view is the more profitable is inclicated by the occurrence of secondary sexual characters in the lower forms, as in the insects and crustaceans, in which it appears almost inconceivable that the ornamentation could have been acquired in connection with the asthetic taste of the other sex. It does not seem to me that the conditions in the higher animals call for any other explanation than that which applies to these lower forms.

My position may be summed up in the statement, that, while in some cases there appears to be a connection between the presence in one sex of secondary sexual organs and their effect on the other sex, yet their origin cannot be explained on account of this connection. 


\section{Individual Adjustments as Adaptations}

As pointed out in the first chapter, there is a group of adaptations, obviously including several quite different kinds of phenomena, that can at least be conveniently brought together under the general rubric of individual adjustments or regulations. A few examples of these will serve to show in what sense they may be looked upon as adaptations, and how they may be regarded from the evolutionary point of view.

\section{Color Changes as Individual Adaptations}

The change in color of certain fish in response to the color of the background, the change in color of some chrysalides also in response to their surroundings, appears to be of some use to the animals in protecting them from their enemies. The change in color from green to brown and from brown back to green in several lizards and in some tree frogs is popularly supposed to be in response to the color of the surroundings, but a more searching examination has shown that, in some cases at least, the response has nothing to do with the color of the background.

In the first cases mentioned above, in which the response appears to be of some advantage to the animal, the question may be asked, how have such responses arisen? The selection theory assumes that those animals that responded at first to a slight degree in a favorable direction have escaped, and this process being repeated, the power to change has been gradually built up. The mutation theory will also account for the result by assuming the response to have appeared as a new quality, but it has been preserved, not because it has been of vital importance to its possessor, but simply because the species possessing it has been able to survive, perhaps in some cases even more easily, although this is not essential. Even if the change were of no direct benefit, 
or even injurious to a slight degree, it might have been retained, as appears in fact to be the case in the change of color of the green lizards.

\section{Increase of Organs through Use and Decrease Through Disuse}

We meet here with one of the most characteristic and unique features of living things as contrasted with non-living things. We shall have to dismiss at once the idea that we can explain this attribute of organisms by either the selection or the mutation theory; for we find animals possessing this power that could never be supposed to have acquired it by any experience to which they have been subjected; and since it appears to be so universally present, we cannot account for it as a chance mutation that may have appeared in each species. No cloubt Wolff had responses of this kind in view when he made the rather sweeping statement that purposeful adaptation is the most characteristic feature of living things. The statement appears to contain a large amount of truth, if confined to the present group of phenomena.

This power of self-regulation may confer a great benefit on its possessor. The increase in the size and strength of the muscles through use may give the animal just those qualities that make its existence easier. The increase in the power of vision, or at least of visual discrimination through use, of the power of smell and of taste, of hearing and of touch, are familiar examples of this phenomenon.

However much we may be tempted to speculate as to how this property of the animal may have been acquired, we lack the evidence which would justify us in formulating even a working hypothesis. It may be that when we come to know more of what the process of contraction of the muscle involves, the possibility of its development as a consequence 
of its use may be found to be a very simple phenomenon that requires no special explanation at all to account for its existence in the individual, further than that the muscles are of such a kind that this is a necessary physical result of their action. But until we know more of the physiology involved in the process, it is idle to speculate about the origin of the phenomenon.

\section{Reactions of the Organism to Poisons, etc.}

In this case also we meet with a number of responses for whose origin we can give not the shadow of an explanation. On the other hand, the cases are significant in so far as a number of them show quite clearly that the response cannot have been acquired through the experience of the organism, or the selection of those individuals that have best resisted the particular poison. This is true, because in a number of cases the poison is a substance that the animal cannot possibly have met with during the ordinary course of its life, or of that of its ancestors. It may be argued, it is true, that in the case of the poisons produced by certain bacteria the power of resistance has been acquired through the survival of the less susceptible, or more resistant, individuals. Improbable as this may be in some cases, it does not, even if it were true, alter the real issue, for it can be shown, as has just been said, that the same power of responding adaptively is sometimes shown in cases of poisons that are new to the animal.

There is no question that different individuals respond in very different degrees to these poisonous substances, and it is easy to imagine in the case of contagious diseases that a sort of selective process might go on that would bring the race up to the highest point to which fluctuating variations could be carried, even to complete immunity; but even if this were the case, it seems to be true that the moment 
the selection stopped the race would sink back to the former condition.

All this touches only indirectly the main point that we have under consideration, namely, the existence of this power of resistance in cases where it cannot have been the result of any educative process. Since the responses to new poisons do not appear to be in principle different from the responses to those to which the organism may have possibly been subjected at times in the past, we shall probably not go far wrong if we treat all cases on the same general footing. Whether the power of adaptation to certain substances, such as nicotine, morphine, cocaine, arsenic, alcohol, etc., is brought about by the formation of a counter-substance is as yet unproven. And while it seems not improbable that in some of these instances it may turn out that this is the case, especially for poisons of plant origin, it is better to suspend judgment on this point until each case has been established.

In recent years it has been shown that the animal body has the power of making counter-substances when a very large number of different kinds of things are introduced into the blood. We seem to be here on the threshold of a field for discovery which may, if opened up, give us an insight into some of the most remarkable phenomena of adaptation shown by living things.

It has already been pointed out that it appears to be almost a reductio ad absurdum to speak of animals adapting themselves to poisonous substances. It is curious, too, that in man at least the use of these substances may arouse a craving for the poison, or at any rate the individual may become so dependent on the poison that the depression following its disuse may lead to a clesire for a repetition of the close. The two questions that are raised here must be kept apart, for the adaptation of the indiviclual to the poison and the socalled craving for it may depend on quite different factors. 
Nevertheless, it seems to be true in the case of morphine and of arsenic, and probably for some other substances as well, that if their use is suddenly stopped the individual may die in consequence. In this respect the organism behaves exactly as it does to an environment to which it has become adapted.

\section{REgENERATION}

Many animals are able to replace lost parts, and all of them can heal wounds and mend injuries. This power is obviously of great advantage to them, and it has been supposed by Darwin, and more especially by his followers, that the power has been acquired through natural selection. It is not difficult to show that regeneration could not, in many cases, and presumably in none, have been acquired in this way. Since I have treated this subject at some length recently in my book on "Regeneration," I shall attempt to do no more here than indicate the outline of the argument.

The Darwinians believe that, if some individuals of a species have the power to replace a part that is lost better than have other inclividuals, it would follow that those would survive that regenerate best, and in this way after a time the power to regenerate perfectly would be acquired.

But the matter is by no means so simple as may appear from this statement. In the first place, it is a matter of common observation that all the individuals of a species are never injured in the same part of the body at the same time. In those cases in which it is known that a special part is often injured, an examination has shown that there are not more than ten per cent of individuals that are injured at any one time, and in the case of the vast majority of animals this estimate is much too great. Thus there will be very little chance for competition of the injured individuals in each generation with each other, and the effects that are imagined to be gained as a result would be entirely lost 
by crossing with the uninjured individuals. But it is not necessary to consider this possibility, since there is another fact that shows at once that the power to regenerate could not have been gained through selection. The number of uninjured individuals in each generation will be much greater than the injured ones, and these will have so great an advantage over the injured individuals that, if competition approached the degree assumed by the selectionists, the injured individuals should be exterminated. A slight advantage gained through better powers of regeneration would be of little avail in competition, as compared with the competition with the uninjured individuals. Since selection is powerless to accomplish its end without competition, and since with competition all the injured individuals would be eliminated, it is clear that an appeal cannot be made to selection to explain the power of regeneration.

In many cases the power of regeneration could not have been slowly acquired through selection, since the intermediate steps would be of no use. Unless, for example, a limb regenerated from the beginning almost completely, the result would be of no use to the animal. If the limb did regenerate completely the first time it was injured, then the selection hypothesis becomes superfluous.

There are also a few cases known in which a process of regeneration takes place that is of no use to the animal. If, for instance, the earthworm (Allolobophora fatida) be cut in two in the middle, the posterior piece regenerates at its anterior cut end, not a head, but a tail. Not by the widest stretch of the imagination can such a result be accounted for on the selection theory. Again, we find the reverse case, as it were, in certain planarians. If the head of Planaria lugubris is cut off just behind the eyes, there develops at the cut surface of this head-piece another head turned in the opposite direction. Here again we have the regeneration of a perfect structure, but one that is entirely useless to the in- 


\section{Origin of Different Kinds of Adaptations $38 \mathrm{I}$}

dividual. The development of an antenna in place of an eye in the shrimp, when the eye stalk is cut off near its base, is another instance of the occurrence of a perfectly constant process, but one that is of no use to the organism.

When we recall that in some organisms regeneration takes place in almost every part of the body, it does not seem possible that this power could have been acquired by selection. And when we find that many internal organs regenerate, that can rarely or never be injured without the animal perishing, it seems impossible that this can be ascribed to the principle of natural selection.

It has also been found that if the first two cells of the egg of a number of animals, jellyfish, sea-urchins, salamanders, etc., be separated, each will produce an entire animal. In some of these cases it is inconceivable that the process could ever have been acquired through selection, because the cells themselves can be separated only by very special and artificial means.

These, and other reasons, indicate with certainty that regeneration cannot be explained by the theory of natural selection. 


\section{CHAPTER XI}

\section{TROPISMS AND INSTINCTS AS ADAPTATIONS}

OF the different kinds of adaptation none are more remarkable than those connected with the immediate responses of organisms to external agents. These responses are usually thought of as associated with the nervous system; and while in the higher forms the nervous system plays an important rôle in the reaction, yet in many cases it is little more than the shortest path between the point stimulated and the muscles that contract; and in the lower animals, where we find just as definite responses, there may be no distinct nervous system, as in the protozoa, for instance.

Many of the so-called instincts of animals have been shown in recent years to be little more than direct responses to external agents. Many of these instincts are for the good of the individual, and must be looked upon as adaptations. For example: if a frog is placed in a jar of water, and the temperature of the water lowered, the frog will remain at the top until the water reaches 8 degrees $C$., when it will dive down to the bottom of the jar; and, if the temperature is further lowered, it will remain there until the water becomes warmer again, when it will come to the surface again. It is clear that, under the ordinary conditions of life of the frog, this reaction is useful to it, since it leads the animal to go to the bottom of the pond on the approach of cold weather, and thus to avoid being frozen at the surface.

Another illustration of an instinct that is a simple response to light is shown by the earthworm. During the day the worm remains in its burrow, but on dark nights it comes out 
of its hole, and lies stretched out on the surface of the ground. It procures its food at this time, and the union of the inclividuals takes place. In the early morning the worm retires into its burrow.

This habit of the earthworm is the direct result of its reaction to light. It crawls away from ordinary light as bright as that of diffuse daylight, and, indeed, from light very much fainter than that of daylight. If, however, the light be decreased to a certain point, the worm will then turn and crawl toward the source of light. This lower limit has been found by Adams to be about that of .oo c candle-metre. This corresponds to the amount of light of a dark night, and gives an explanation of why the worm leaves its burrow only at night, and also why it crawls back on the approach of dawn. It is also obvious that this response is useful to the animal, for if it left the burrow during the day, it would quickly fall a prey to birds.

The blow-fly lays its eggs on decaying meat, on which the larvæ feed. The fly is drawn to the meat by its sense of smell, a simple and direct response to a chemical compound given off by the meat. The maggot that lives in the decaying meat is also attracted by the same odor, as Loeb has shown, and will not leave the meat, or even a spot on a piece of glass that has been smeared with the juice of the meat, so long as the odor remains. Here again the life of the race depends on the proper response to an external agent, and the case is all the more interesting, since the response of the fly to the meat is of no immediate use to the fly itself, but to the maggot that hatches from the egg of the fly.

The movement toward or from a stimulating agent is, in some cases, brought about in the following way. Suppose an earthworm is lying in complete darkness, and light be thrown upon it from one side. The worm turns its head, as it thrusts it forward, to the side away from the light; and 
as it again moves forward, it continues to bend its head away from the light, until it is crawling directly away from the source. When the light first strikes the worm, the two sides will be differently illuminated. This causes a bending of the head, as it stretches forward, toward the side of less illumination, and the bending is due to a stronger contraction of some of the muscles on the less illuminated side; at least the reaction appears to be due to a simple response of this kind. When the body has been so far turned that the two sides are equally illuminated, the muscles of the two sides will contract equally, and the movement will be straight forward and away from the light. If the reaction is as simple as this (which is in principle the explanation advanced by Loeb), the result is a simple reflex act, and need not involve any consciousness or intentional action on the part of the worm to crawl away from the light. In fact, the same reaction takes place when the brain is removed, not so quickly or definitely, it is true, but this may be due to the removal of the anterior segments of the worm, in which part the skin appears to be more sensitive to light than elsewhere.

Another factor that plays an important rôle in the habits of the earthworm is the response to contact, - the so-called stereotropism. If, in crawling over a flat surface, the worm comes in contact with a crevice, it will crawl along it, and refuse to leave until the end is reached. The contact holds the worm as strongly as though it were actually pulled into the crevice. It can be forced to leave a crevice only by strong sunlight, and then it does not do so at once. If the worm crawls into a small glass tube, it is also held there by its response to contact, and the smaller the tube, the more difficult is it to make the worm leave by throwing strong sunlight upon it.

Loeb has found that when winged aphids, the sexual forms, are collected in a tube, and the tube is kept in a room, the aphids crawl toward the light. This happens in 
ordinary diffuse light, as well as in lamplight. It is stated that the animals orientate themselves towards the light more quickly when it is strong than when it is weak. They turn their bodies toward the light, and then move forward in the direction from which the rays come. It can be shown by a simple experiment that the aphids are turned by the dircction of the light, and not by its intensity. If they are placed in a tube, and the tube laid obliquely before a window in such a way that the direct sunlight falls only on the inner end of the tube, the aphids will, if started at the inner end of the tube, first crawl toward the outer surface of the tube, and then wancler along this wall, passing out of the region of sunlight into the end of the tube nearest the window, where they come to rest at the end. They have moved constantly towards the direction from which the rays come, passing, as it were, from ray to ray, but each time toward a ray nearer the source of the light.

If the tube be turned toward the window, and the window end be covered with blue glass, the aphids crawl into this end of the tube, as they would have clone had the tube been uncovered. If, on the other hand, the end of the tube be covered with red glass, they do not crawl into the part of the tube that is covered, unless they are very sensitive to light. Even in the latter case they may remain scattered in the red part, and do not all accumulate at the end, as they do when blue glass is used. In other words, while they respond to blue as they do to ordinary light, they behave toward red as they do towards a very faint light.

In diffuse daylight the aphids, as has been said, crawl toward the light, but if they come suddenly into the sunlight they begin to fly. Thus they remain on the food plant until the sun strikes it, and then they fly away.

The aphid also shows another response; it is negatively geotropic, i.e. it tends to crawl upward against gravity. If placed on an inclined, or on a vertical, surface, it will crawl 
upward. Such an experiment is best made in the dark, since in the light the aphid also responds to the light. If put on a window it crawls upward never downward.

Aphids are also sensitive to heat. If they are placed in a darkened tube and put near a stove, they crawl away from the warmer end; but if they are acted upon by the light at the same time, they will be more strongly attracted by the light than repulsed by the heat. We thus see that there are at least three external agents that determine the movements of this animal, and its ordinary behavior is determined by a combination of these, or by that one that acts so strongly as to overpower the others.

The swarming of the male and female ants is also largely directed by the influence of light. Loeb observed that when the direct sunlight fell full upon a nest in a wall the sexual forms emerged, and then flew away. Other nests in the ground were affected earlier in the day, because the sun reached them first. These ants, when tested, were found to respond to light in the same way as do the aphids. The wingless forms, or worker ants, do not show this response, and the winged forms soon lose their strong response to light after they have left the nest. Thus we see that the heliotropism is here connected with a certain stage in the development of the individual; and this is useful to the species, as it leads the winged queens and males to leave the nest, and form new colonies. Even the loss of response that takes place later may be looked upon as beneficial to the species, since the queens do not leave the nest after they have once established it.

It is familiar to every one that many of the night-flying insects are attracted to a lamplight, and since those that fly most rapidly may be actually carried into the flame before they can turn asicle, it may seem that such a response is worse than useless to them. The result must be considered, however, in connection with other conditions of their life. 
The following experiments carried out by Loeb on moths show some of the responses of these insects to light.

Night-flying moths were placed in a box and exposed in a room to ordinary light. As twilight approached the moths became active and began to fly always toward the window sicle of the box. They were positively heliotropic to light of this intensity. If let out of the case, they flew toward the window, where they remained even during the whole of the next day, fully exposed to light. If the moth is disturbed in the daytime, so that it flies, it goes always toward the light, and never away from it. These facts show that the moth is always positively heliotropic, and also that the flight toward the lamp is a natural response, misapplied in this case. That the moths do not fly by day is due to another factor, namely, the alternation in the degree of their sensitiveness at different times. But this condition alone does not seem to account fully for all the facts.

If the moths are given the alternative of flying toward the evening light, or toward the lamp, they always go toward the brighter light. Thus if, when they swarm at dusk, they are set free in the middle of the room, at the back of which a lamp is burning, the moths fly toward the window. If, however, they are set free within a metre of the lamp, they fly toward it.

The explanation that Loeb offers of the habit of these moths to fly only in the evening is, that, although they are at all times positively heliotropic, they respond to light only in the evening. In other words, it is assumed that there is a periodic change in their sensitiveness to light, which corresponds with the change from day to night. Loeb says that, just as certain flowers open only at night, and others only during the day, so do moths become more responsive in the evening, and butterflies during the day. Both moths and butterflies are positively heliotropic, and the sensitiveness of moths to light may be even greater in the evening than is 
that of butterflies, for the light of the evening to which the moth reacts is less than the minimal to which the butterfly responds.

Moths appear to pass into a sort of sleep during the day, while butterflies are quiescent only at night. The periodicity of the sleeping time continues, at least for several days, when the insects are kept in the dark. For instance, moths kept in the dark become restless as the evening approaches, as Réaumur observed long ago. It has been found in plants that this sort of periodicity may continue for several days, but gradually disappears if the plants are kept in the dark. By using artificial light, and exposing the plants to it during the night, and putting them in the dark during the day, a new periodicity, alternating with the former one, may be induced; and this will continue for some days if the plants are then kept continually in the dark.

Loeb tried the experiment of exposing the quiescent moths suddenly to a lower intensity of light, in order to see if they would respond equally well at any time of day. It was found that if the change was made in the forenoon, between six o'clock and noon, it was not possible to awaken the moths by a sudden decrease in the intensity of the light. But it was possible to do so in the afternoon, long before the appearance of dusk. It appears, therefore, that in this species, Sphinx cuphorbice, it is possible to infuence the period of awakening by decreasing the intensity of light, but this can be done only near the natural period of awakening. It seems to me that this awaking of a positively heliotropic animal by decreasing the light needs to be further investigated.

The day butterflies are also positively heliotropic. Butterflies of the species Papilio machaon, that have been raised from the pupa, remain quietly on the window in the diffuse daylight of a bright day. They can be carried around on the finger without leaving it, but the moment they come into the direct rays of the sun they fly away. 


\section{Tropisms and Instincts as Adaptations $3^{89}$}

Butterflies that have just emerged from their pupa case exhibit a marked negative geotropic reaction, and this appears to be connected with the necessity of unfolding their wings at this time. Loeb says that the same cause that determines the direction of the falling stone and the paths of the planets, namely, gravity, also directs the actions of the butterfly that has just left its pupa case. The geotropic response is especially strong at first. The animal wanders around until it reaches a vertical wall, which it immediately ascends, straight upward, and remains hanging at the top until its wings have unfolded. A similar response occurs in the final stage of the larva of the May-fly, which leaves the water and crawls up a blade of grass, or other vertical support, and there, bursting the pupa skin, it dries its wings and flies away. That this is a reaction to gravity and not to light is shown by Loeb's observation, that their empty skins are sometimes observed under a bridge where the light does not come from above. "This observation on the larva of the May-fly contradicts the assumption that the 'purpose' of the geotropic response of the butterfly is that it may the better unfold its new wings, for in the ephemerid larva the negative geotropism appears at a time when no wings are present." On the other hand, it should not be overlooked that the reaction is important for the May-fly larva in other ways, because it leads the larva to leave the water at the right period, and come out into the air, where the flying insect can more safely emerge.

It is not without interest to find that caterpillars exhibit some of the same reaction shown by butterflies. Loeb has made numerous experiments with the caterpillars of Porthesia chrysorhea. The caterpillars of this moth collect together in the autumn and spin a web or nest in which they pass the winter. If they are taken from the nest and brought into a warm room, they will orientate themselves to the light, and also crawl toward it. If 
placed in a tube, they crawl to the upper side of the glass and then along this side toward the light. If a covering is placed over the end of the tube that is turned toward the window, the caterpillars will crawl only as far as the edge of the cloth. They also react negatively to gravity. If kept in a dark room, they will crawl upward to the top of the receptacle in which they are enclosed. If subjected to the influences of both light and gravity, they respond more strongly to the light. The caterpillars also show a contact reaction. They tend to collect on convex sides or on corners and angles of solid bodies. They may even pile up one on top of the other in response to this reaction; the convex side of a quiescent animal acting on another animal crawling over it as any convex surface would do and holding the animal fast.

These three kinds of reactions determine the instincts of these caterpillars. In the spring, when they become warm, they leave the nest. Positive heliotropism and negative geotropism compel them to crawl upward to the tops of the branches of the trees, and there the contact reaction with the small buds holds them fast in this place. That they are not attracted to the end of the branches by the food that they find there is shown by placing buds in the bottom of the tubes in which the caterpillars are contained. The caterpillars remain at the top of the tube, although food is within easy reach. If, however, they are placed directly on the buds, the contact reaction will hold them there, and they will not crawl farther upward. Curiously enough, as soon as the caterpillars have fed and the time for shedding approaches, the responsiveness to light and to gravity decreases, and at the time of shedding they do not respond at all to these agents. These same caterpillars react also to warmth above a certain point. In a dark tube placed near a store, the caterpillars collect at the end farthest away from the source of the heat. They react to light best at a temperature between 20 and 30 
degrees C., and above this temperature point they become restless and wander about.

The very close connection between the reactions of this caterpillar and its mode of life is perfectly obvious. The entire series of changes seems to have for its "purpose" the survival of the individual by bringing it to the place where it will find its food. It may seem natural to conclude that these responses have been acquired for this very purpose, but let us not too quickly jump at this obvious conclusion until the whole subject has been more fully examined.

The upward and downward movements of some pelagic animals have been shown to depend on certain tropic responses. Every student of marine zoology is familiar with the fact that many animals come to the surface at night, and go down at the approach of daylight. It has been shown that this migration is clue largely to a response to light. Light can penetrate to only about four hundred metres in sea-water, and there is complete darkness below this level. It has been shown that the swimming larvæ of one of the barnacles is positively heliotropic in a weak light, but negatively heliotropic in a stronger light. Animals having responses like these will come to the surface as the light fades away in the evening and remain there until the light becomes too bright in the following morning. They will then become negatively heliotropic and begin to go down. When they reach a level where the intensity of the light is such that they become positively heliotropic, they will turn and start upward again. Thus during the clay they will keep below the surface, remaining in the region where they change from positive to negative, and vice versa.

It would not be difficult to imagine that this upward and downward migration of pelagic animals is useful to them, but, on the other hand, it may be equally well imagined that the response may be injurious to them. Thus it might be supposed that certain forms could procure their food by coming 
to the surface at night, and avoid their enemies by going down during the day. But it is difficult to see why organisms that serve as prey should not have acquired exactly the opposite tropisms in order to escape.

Some of these marine forms are also geotropic. Loeb has determined that "the same circumstances that make the animals negatively heliotropic also make them positively geotropic, and vice iersa." It was found, for instance, that the larva of the marine worm Polygordius is negatively geotropic at a low temperature, while at a higher temperature it is positively geotropic. This response would drive the animals upward when the water becomes too cold, and back again if the surface water becomes too warm; but whether the response is so adjusted that the animals keep, as far as possible, in water of that temperature that is best for their development, we do not know. We can easily imagine that within wide limits this is the case.

The change from positive to negative can also be brought about in other ways. One of the most striking cases of this sort is that described by Towle in one of the small crustaceans, Cypridopsis ailua. It was found that after an animal had been picked up in a pipette its response was always positive; that is, it swam toward the light, no matter what its previous condition had been. The disturbance caused by picking the animal up induced always a positive response towards light. If the light were moved, the Cypridopsis followed the light. In this way it could be kept positive for some time, but if it came to rest, or if it came into contact with the sides or end of the trough, it became, after a short time, negatively heliotropic, and remained negative as long as it could be kept in motion, without being disturbed, or coming into contact with a solid object. If when positive it were allowed to reach the glass at the end of the trough, it would swim about there, knocking against the glass, and then soon turn and swim away from the light. If the light were 
shifted while the negative animal was in the middle of the trough, it would turn and swim directly away, as before, from the source of light. It could be kept in this negative state as long as it did not come into contact with the ends.

It appears that the positive condition in Cypridopsis is of short duration, and ceases after a while either as a response to contact or without any observable external factor causing the change.

This crustacean lives at the bottom of pools, amongst waterplants, and here also, no doubt, the same change from one to the other reaction takes place. What possible advantage it may be to the animal to be kept continually changing in this way is not at all obvious, nor, in fact, are we obliged to assume that this reaction may be of any special use to it. Indeed, it is far from obvious how the change that causes the animal to swim toward the light when it is disturbed could be of the least advantage to it.

In another crustacean, one of the marine copepods, Labidocera astiva, it has been shown by Parker that the male and female react in a somewhat different way both to light and to gravity. The females are strongly negatively geotropic, and this sends them up to the top of the water. The males are very slightly negatively geotropic. The females are strongly positively heliotropic toward light of low intensity; the males show the same response to a less degree. To strong light the females are negative and the males are indifferent. On the other hand, the males are attracted to the females, probably in response to some chemical substance diffusing from the females, since the males show the same reaction when the females are enclosed in an opaque tube through whose ends a diffusion of substances may take place. This crustacean frequents the surface of the ocean from sunset to sunrise. During the day it retires to deeper water. Its migrations can be explained as follows: The females come to the surface at 
night, because they are positively heliotropic to weak light, and also because they are negatively geotropic. They go down during the day, because they react to bright light more strongly than to gravity. The males follow the females, largely because they react positively chemotactically toward the females.

Some other animals respond in a somewhat different way to light, as shown by the fresh-water planarians. These animals remain during the day under stones, where the amount of light is relatively less than outside. If they are placed in a dish in the light in front of a window, they crawl away from the light, but when they reach the back of the dish they do not come to rest, but continue to crawl around the sides of the dish even toward the light. The light makes the worms restless, and while they show a negative response as long as they are perfectly free to move away from the light, they will not come to rest when they come to the back of the dish if they are there still in the light, because the irritating action of the light on them is stronger than its clirective action. If, however, in crawling about they come accidentally into a place less bright than that in which they have been, they stop, and will not leave this somewhat darker spot for a brighter one, although they might leave the newly found spot for one still less bright. At night the planarians come out and wander around, which increases their chance of finding food, although it would not be strictly correct to say that they come out in search of food. If, however, food is placed near them, a piece of a worm, for example, they will turn toward it, being directed apparently by a sense of smell, or rather of taste.

The heliotropic responses of the planarians appear to be of use to them, causing them to hide away in the daytime, and to come out only after dark, when their motions will not dis; cover them to possible enemies. But some of the planarians 
are protected in other ways, so that they will not be eaten by fish, probably owing to a bad taste; so that it is not so apparent that they are in real need of the protection that their heliotropic response brings to them. Their turning towards their food is, however, beyond question of great advantage to them, for in this way they can find food that they cannot detect in any other way.

The unicellular plants were amongst the first organisms whose tropic responses were studied, and the classical work of Strasburger gave the impetus to much of the later work. In recent years the unicellular animals, the protozoans, have been carefully studied, more especially by Jennings. $\mathrm{His}$ results show that the reactions in these animals are different in some important respects from those met with in higher forms. For instance, most of the freeswimming infusoria are unsymmetrical, as are also many of the flagellate forms, and as they move forward they rotate freely on a longitudinal axis. It is therefore impossible that they could orientate themselves as do the higher animals that have been described above, and we should not expect these Protozoa to react in the same way. In fact, Jennings shows that they exhibit a different mode of response. Paramocium offers a typical case. As it moves forward it rotates toward the aboral side of the body. As a result of the asymmetry of the body, the path followed, as it revolves on its own axis, is that of a spiral. Did the animal not rotate, as it swims forward, its asymmetrical form would cause it to move in a circle, but its rotation causes, as has been said, the course to be that of a spiral, and the general direction of movement is forward. ${ }^{1}$ The rotation of a paramœcium on its axis is in turn caused by the oblique stroke of the cilia that cover the surface of the body. Their action when reversed causes the animal to rotate backward.

'The same result is attained by a bullet that is caused by the rifling to rotate as it moves forward. 
If a drop of weak acid be put into the water in which the paramœcia are swimming, - for instance, in the water between a cover-slip and a slide, - it will be found, after a time, that many individuals have collected in the drop. It was at first supposed that the paramœecia are attracted by the diffusion of the acid in the water, and turn toward the source of the chemical stimulus; but Jennings has shown that this is not the way in which the aggregation is brought about. If the individuals are watched, it will be found that they swim forward in a spiral path without regard to the position of the drop of acid. If one happens, by chance, to run into the drop, there is no reaction as it enters, but when it reaches the other side of the drop, and comes into contact with the water on this side, it suddenly reacts. It stops, backs into the middle of the drop, rotates somewhat toward the aboral side (i.e. away from the restibule), and then starts forward again, only to repeat the action on coming into contact with the edge of the drop again. The paramocium has been caught in a veritable trap. All paramoecia that chance to swim into the drop will also be caught, until finally a large number will accumulate in the region. The result shows, that, in passing from ordinary water into a weak acid, no reaction takes place; but having once entered the acid, the animal reacts on coming into contact with the water again.

On the other hand, there are some substances to which the paramocium may be said to be negatively chemotropic. If a drop of a weak alkaline solution be put into water in which paramocium is swimming, an individual that happens to run against it reacts at once. It stops instantly, backs off, revolving in the opposite direction, turns somewhat to one side, and swims forward again. The chances are that it will again hit the drop, in which case it repeats the same reaction, turning again to one side. If it continues to react in this way, it will, in the course of time, turn so far that when it swims forward it will miss the edge of the drop, and then continue 
on its way. If an individual were put into an alkaline drop, it would leave it, because it would not react when it passed from inside the drop into the surrounding water.

Unicellular animals react to other things besides differences in the chemical composition of different parts of a solution. In many cases they react to light, swimming toward or away from it according to whether they are positively or negatively heliotropic. If they are positively heliotropic, and while swimming run into a shadow, they react as they would on coming into contact with a drop of acid. Since they rotate as they swim forward, we cannot explain their orientation as in the case of other animals that hold a fixed vertical position. If we assume that the two ends of the body are differently affected by the light, for which there is some evidence, we can perhaps in this way account for their turning toward, or away from, the source of light.

Changes in the osmotic pressure of the different parts of the fluid, mechanical stimulation produced by jarring, extremes of heat and of cold, all cause this same characteristic reaction in Paramœcium; and this accounts for their behavior toward these agents that are so different in other respects.

Paramœcia, as well as other protozoans, show a contact response. They fix themselves to certain kinds of solid bodies. If, for example, a small bit of bacterial slime is put into the water, the paramœcia collect around it in crowds, and eat the bacteria; but they will collect in the same way around almost any solid. On coming in contact with bodies having a certain physical texture, the cilia covering the paramœcium stop moving, only those in the oral groove continuing to strike backward. The animal comes to rest, pressed against the solid body. If one or more paramœcia remain in the same place, they set free carbon dioxide, as a result of their respiratory processes. There is formed around them a region containing more of this acid than does the surround- 
ing water. If other moving paramœcia swim, by chance, into this region, they are caught, and as a result an accu:mulation of individuals will take place. The more that collect the larger will the area become, and thus large numbers may be ultimately entrapped in a region where there is formed a substance that, from analogy with other animals, we should expect to be injurious.

The question as to how far these responses of the unicellular forms are of advantage to them is difficult to decide, for while, as in the above case, the response appears to be injurious rather than useful, yet under other conditions the same response may be eminently advantageous. In other cases, as when the paramœecia back away, and then swim forward again, only to repeat the process, the act appears to be such a stupid way of avoiding an obstacle that the reaction hardly appears to us in the light of a very perfect adaptation. If we saw a higher animal trying to get around a wall by butting its head into it until the end was finally reached, we should probably not look upon that animal as well adapted for avoiding obstacles.

Bacteria, which are generally looked upon as unicellular plants, appear, despite the earlier statements to the contrary, to react in much the same way as do the protozoans, according to the recent work of Rothert, and of Jennings and Crosby. The bacteria do not seem to turn toward or away from chemical substances, but they collect in regions containing certain substances in much the same way as do the protozoans. The collecting of bacteria in regions where oxygen is present has been known for some time, but it appears from more recent results that they are not attracted toward the oxygen, but by accidentally swimming into a region containing more oxygen they are held there in the same way as is paramocium in a drop of acid. On the other hand bacteria do not enter a drop of salt solution, or of acids, or of alkalies. They react negatively to all such substances. Some kinds of 
bacteria have a flagellum at each end, and swim indifferently in either direction. If they meet with something that stimulates them, as they move forward, they swim away in the opposite direction, and continue to move in the new direction until something causes again a reversal of their movement. In this respect their mode of reaction seems of greater advantage than that followed by paramœcium.

Another instinct, that appears to be clue to a tropic response, is the definite time of day at which some marine animals cleposit their eggs. The primitive fish, Amphioxus, sets free its eggs and sperm only in the late afternoon. A jellyfish, Gonionema, also lays its eggs as the light begins to grow less in the late afternoon, and in this case it has been found that the process can be hastened if the animals are placed in the dark some hours before their regular time of laying. There is no evidence that this habit is of any advantage to the animal. We may imagine, if we like, that the early stages may meet with less risk at night, but this is not probable, for it is at this time that countless marine organisms come to the surface, and it would seem that the chance of the eggs being destroyed would then be much greater. It is more probable that the response is of no immediate advantage to the animals that exhibit it, although in particular cases it may happen to be so.

This response recalls the diurnal opening and closing of certain flowers. The flowers of the night-blooming cereus open only in the clusk of evening, and then emit their strong fragrance. Other flowers open only in the daytime, and some only in bright sunlight. It is sometimes pointed out that it is of advantage to some of these flowers to open at a certain time, since the particular insects that are best suited to fertilize them may then be abroad. This may often be the case, but we cannot but suspect that in other cases it may be a matter of little importance. In special instances it may be that the time of opening of the flowers is of importance 
to the species; but even if this is so, there is no need to assume that the response has been gradually acquired for this particular purpose. If it were characteristic of a new form to open at a particular time, and there were insects in search of food at this time that would be likely to fertilize the plant, then the plant would be capable of existing; but this is quite different from supposing that the plant developed this particular response, because this was the most advantageous time of day for the fertilization of its flowers.

We can apply this same point of view, I believe, to many of the remarkable series of tropisms shown by plants, whose whole existence in some cases is closely connected with definite reactions to their environment. Let us examine some of these cases.

When a seed germinates, the young stem is negatively geotropic, and, in consequence, as it elongates it turns upward towards the light that is necessary for its later growth. The root, on the contrary, is positively geotropic, and, in consequence, it is carried downward in the ground. Both responses are in this case of the highest importance to the seedling, for in this way its principal organs are carried into that environment to which they are especially adapted. It matters very little how the seed lies in the ground, since the stem when it emerges will grow upward and the root downward. The young stem, when it emerges from the soil, will turn toward the light if the illumination comes from one sicle, and this also may often be of advantage to the plant, since it turns toward the source from which it gets its energy. The leaves also turn their broad surfaces toward the light, and as a result they are able to make use of a greater amount of the energy of the sunlight. The turning is due to one side of the stem growing more slowly than the opposite side, and it is true, in general, that plants grow faster at night than in the daylight. Very bright light will in some cases actually stop all growth for a time. Thus we 
see that this bending of the stem toward the light and the turning of the leaves to face the light are only parts of the general relation of the whole plant toward the light.

Negative heliotropism is much less frequent in plants. It has been observed in aërial roots, in many roots that are ordinarily buried in the ground, in anchoring tendrils that serve as holdfasts, and even in the stems of certain climbers. In all of these cases, and more especially in the case of the climbers, the reaction is obviously of advantage to the plant; and it is significant to find, in plants that climb by tendrils carrying adhering disks, that there is a reversal of the ordinary heliotropism shown by homologous organs in other plants. There is an obvious adaptation in the behavior of the tendril, since its growth away from the more illuminated side is just the sort of reaction that is likely to bring it into contact with a solid body.

In this connection it is important to observe that these reactions to light are perfectly definite, being either positive or negative under given conditions, and therefore there is at present nothing to indicate that there has been a gradual transformation from positive to negative, or vice versa. It seems to me much more probable that when the structural change took place, that converted the plant into a climber, there appeared a new heliotropic response associated with the other change. In other words, both appeared together in the new organ, and neither was gradually acquired by picking out fluctuating variations.

The leaves of plants also show a sort of transverse heliotropic response. It has been found, for example, that the leaves of Malva will turn completely over if illuminated by a mirror from below. A curious case of change of heliotropism is found in the flower stalks of Linaria. They are at first positively heliotropic, but after the flower has been fertilized the stalk becomes negatively heliotropic. As the stalks continue to grow longer, they push the fruits into the crevices of the rocks on 
which the plants grow, and in this way insure the lodgement of the seeds. Here we have an excellent example showing that the negative heliotropism of the flower stalk could scarcely have been acquired by slight changes in the final direction, for only the complete change is useful to the plant. Intermediate steps would have no special value.

As has been pointed out in the case of the seedling plant, the main stem responds positively and the roots negatively to gravity. In addition to this, the lateral position taken by the lateral roots and branches and by underground stems are also, in part, due to a geotropic response. In this case also the effect is produced by the increased growth on the upper side when the response is positive, and on the lower when it is negative. Leaves also assume a transverse position in response to the action of gravity, or at least they make a definite angle with the clirection of its action.

The most striking case of geotropic response is seen in plants that climb up the stems of other plants. The twining around the support is the result of a geotropic response of the sides of the stem. The young seedling plant stands at first erect. As its end grows it begins to curve to one side in an oblique position, and this is due to an increase in growth on one side of the apex of the shoot. As a result the stem bends toward the other side. Not only does the end "sweep round in a circle like the hands of a watch," but it rotates on its long axis as it revolves. As a result of this rotation "the part of the stem subjected to the action of the lateral geotropism is constantly changing; and the revolving movement once begun, must continue, as no position of equilibrium can be attained." This movement will carry the end around any support, not too thick, that the stem touches.

Most climbers turn to the left, i.c. against the hands of a watch, others are dextral, and a few climb either way. ${ }^{1}$

1 These cases recall the spiral growth of the shell of the snail, but the spiral in the latter is clue to some other factor. 
Strasburger states that whenever any external force, or substance, is important to the vital activity of the plant or any of its organs, there will also be found to be developed a corresponding irritability to their influence. Roots in dry soil are diverted to more favorable positions by the presence of greater quantities of moisture. This may, I venture to suggest, be putting the cart before the horse. The plant may be only able to exist whose responses are suited to certain external conditions, and these determine the limits of distribution of the plant or the places in which it is found.

A number of plants climb in a different way, and show another sort of tropism. Those that climb by means of tendrils twist their tendrils about any support that they happen to come in contact with, and thus the plant is able to lift its weak stem, step by step, into the air. The twining of the tendrils is due to contact, which causes a cessation of growth at the points of contact. The growth of the opposite side continues, and thus the tendril bends about its support. In the grape and in ampelopsis the tendril is a modified branch. The stalk of the leaves in a few plants, as in Lophospermum, act as tendrils. Other climbers are able to ascend vertical walls owing to the presence of disks, whose secretions hold the tendril firmly against the support, as in ampelopsis.

It is interesting to find in practically all these cases that, whatever the stimulus may be, the results are reached in the same way, namely, by one part growing faster than another. The fact of importance in this connection is that the plant is so constructed that the response is often beneficial to the organism.

Before leaving this subject there is one set of responses to be referred to that is not the result of growth. Certain morements are brought about by the change in the turgidity of certain organs. The small lateral leaflets of Desmodimm gyrans make cirching movements in one to three minutes. No apparent benefit results from their action. The terminal 
leaflets of Trifolim pratense oscillate in periods of two to four hours, but do so only in the dark; in the light the leaflets assume a rigid position. There is nothing in the process to suggest that the movement is useful to the plant, and yet it appears to be as definite as are those cases in which the response is of vital importance. Had these movements been of use, their origin would, no doubt, have been explained because of their usefulness, and the conclusion would have been wrong.

The leaves of the Mimosa respond, when touched, and it cannot be supposed that this is of any great advantage to the plant. The sleep movements of many plants are also due to the effect of light. In some cases the leaflets are brought together with their upper surfaces in contact with one another; in other cases the lower surfaces are brought together. Darwin supposed that these sleep movements served to protect the leaves from a too rapid loss of heat through radiation, but it has been pointed out that tropical plants exhibit the same responses. We have here another admirable instance of the danger of concluding that because we can imagine an advantage of a certain change, that the change has, therefore, been acquired because of the advantage. In the Mimosa not only do the leaflets close together, but the whole leaf drops down if the stimulus is strong. Other plants also show in a less degree the same movements, Robinia and Oxalis for instance, and certainly in these latter the result does not appear to be of any advantage to the plants.

The preceling account of some of the tropisms in animals and plants will serve to give an idea of how certain movements are direct responses to the environment. Some of the reactions appear to be necessary for the life of the individual, others seem to be of less importance, and a few of no use at all. Yet the latter appear to be as definite and well-marked as are the useful responses. I think the conviction will impress itself on any one who examines 
critically the facts, that we are not warranted in applying one explanation to those responses that are of use, and another to those that are of little or of no value. Inasmuch as the Darwinian theory fails to account for the origin of organs of little or of no value, it is doubtful if it is needed to explain the origin of the useful responses. If, on the other hand, we assume that the origin of the responses has nothing to do with their value to the organism, we meet with no difficulty in those cases in which the response is of little or of no use to the organism. That great numbers of responses are of benefit to the organism that exhibits them can be accounted for on the grounds that those new species, that have appeared, that have useful responses, are more likely, in the long run, to survive, than are those that do not respond adaptively.

VTe may now examine some of the more complicated responses and instincts, more especially those of the higher animals. Some of these are pure tropisms, i.c. definite responses or reactions to an external exciting agent ; others may be, in part, the result of individual experience, involving memory; others, combinations of the two; and still others may depend on a more complex reaction in the central nervous system of the animal. These cases can be best understood by means of a few illustrations.

As an example of a simple action may be cited a wellknown reflex after cutting the nerve-cord of the frog, or after destroying the brain. If the frog is held up, and its side tickled, the leg is drawn up to rub the place touched. To accomplish this requires a beautifully adjusted system of movements, yet the act seems to be a direct reflex, involving only the spinal cord.

An example of a somewhat more complex reflex is the biting off of the navel-string by the mother in rodents and other mammals; an act eminently useful to the young animal, although of no importance to the mother herself. 
The protection of the young by their parents from the attacks of other animals appears to be a somewhat complex instinct, and it is interesting to note that the protection is extended to the young only so long as they are in need of it, and as soon as they are able to shift for themselves the maternal protection is withdrawn.

The instinct of the young chick to seize in its beak any small moving object is a simple and useful reflex action, but if the object should happen to be a bee which stings the chick, another bee or similar insect will not be seized. Here we see that a reflex has been changed, and changed with amazing quickness. Moreover, the chick has learnt to associate this experience with a particular sort of moving object. It is this power to benefit by the result of a brief experience that is one of the most advantageous properties of the organism.

Young chicks first show a drinking reflex if by chance their beaks are wet by water. At once the head is lifted up, and the drop of water passes down the throat. In this way the chick first learns the meaning of water, and no doubt soon comes to associate it with its own condition of thirst. The sight of water produces no effect on the inexperienced chick, and it may even stand with its feet in the water without drinking; but as soon as it touches, by chance, the water with its beak, the reflex, or rather the set of reflexes is started.

A more complicated instinct is that shown by the spider in making its web. In some cases the young are born from eggs laid in the preceding summer, and can have had, therefore, no experience of what a web is like; and yet, when they come to build this wonderfully complex structure, they do so in a manner that is strictly characteristic of the species.

The formation of the comb by bees, in which process. with a minimum of wax, they secure a maximum number of small storehouses in which to keep their honey and rear their young, is often cited as a remarkable case of adaptation. 
There has been some discussion as to whether birds build their nests in imitation of the nest in which they were reared, or whether they do so independently of any such experience. There can be no doubt, however, that in some birds neither memory nor imitation can play any important part in the result, and that they build their nests as instinctively as spiders make webs.

These instincts of spiders, bees, and birds appear to be more complex than the reflexes and tropisms that were first described. Whether they are really so, or only combinations of simple responses, we do not yet know. That they have come suddenly into existence as we now find them does not seem probable, but this does not mean that they must have been slowly acquired as the result of selection. The mutation theory also assumes that the steps of advance may have been small.

Our account may be concluded with the recital of some instincts, chosen almost at random, that serve to show some other adaptations which are the result of these inborn responses.

It is known that ants travel long distances from their nests, and yet return with unerring accuracy. It has been shown that they are able to do this through a marvellous sense of smell. The track left by the ant, as it leaves the nest, serves as a trail in returning to the starting-point. Moreover, it appears that the ant can pick out her own trail, even when it has been crossed by that of other ants. This means that she can distinguish the odor of her own trail from that of other members of the colony. The sense-organs by means of which the odor is detected lie in the antenna. This fact accounts for certain actions of ants that have been described as showing that they have an affection for each other. Two ants, meeting, pat each other with their antennæ. In this way they are quickly able to distinguish members of their own nest from those of other nests. If they are of the same 
nest, they separate quietly; if of other nests, they may fight. If an ant from one nest is put into another nest, it is instantly attacked and killed - an act that appears to be injurious rather than useful, for the ant might become a valuable member of the new colony. If, however, an ant is first immersed in the blood of a member of the community into which she is to be introduced, she will not be attacked, and may soon become a part of the new community. By her baptism of blood she has no doubt acquired temporarily the odor of the new nest, and by the time that this has worn off she will have acquired this odor by association, and become thereby a member of another colony.

Numerous stories have been related of cases in which an ant, having found food, returns to the nest with as much of it as she can carry, and when she comes out again brings with her a number of other ants. This has been interpreted to mean that in some mysterious way the ant communicates her discovery to her fellow-ants. A simpler explanation is probably more correct. The odor of the food, or of the trail, serves as a stimulus to other ants, that follow to the place where the first ant goes for a new supply of the food. The fact that the first individual returns to the supply of food seems to indicate that the ant has memory, and this is obviously of advantage to her and to the whole colony.

The peculiar habits of some of the solitary wasps, of stinging the caterpillar or other insect which they store up as food for their young, is often quoted as a wonderful case of adaptive instinct. The poison that is injected into the wound paralyzes the caterpillar, but as a rule does not kill it, so that it remains motionless, but in a fresh state to serve as food for the young that hatch from the egg of the wasp. A careful study of this instinct by Mr. and Mrs. Peckham has shown convineingly that the act is not carried out with the precision formerly supposed. It had been claimed that the sting is thrust into the caterpillar on the lower side, a ventral 
ganglion being pierced, the poison acting with almost instantaneous effect. But it may be questioned whether this is really necessary, and whether the same end might not be gained, although not quite so instantaneously, if the caterpillar were pierced in almost any other part of the body. Can we be seriously asked to believe that this instinct has been perfected by the destruction of those individuals (or of their descendants) that have not pierced the caterpillar in exactly the middle of a segment of the anterior ventral surface? It seems to me that the argument proves too much from the selectionist's point of view. If the wasp pierced the caterpillar in the middle of its back, we should have passed over the act without comment; but since the injection is usually made on the ventral side, and since we know that the nervous system lies in this position, it has been assumed that the act is carried out in this way, in order that the poison may penetrate the nervous system more quickly. Yet a fuller knowledge may show that there is really no necessity for such precision.

A curious response is the so-called death-feigning instinct shown by a number of animals, especially by certain insects, but even by some mammals and birds. Certain insects, if touched, draw in their legs, let go their hold, and fall to the ground, if they happen to be on a plant. It is not unusual to meet with the statement that this habit has been acquired because it is useful to the insect, since it may often escape in this way from an enemy. This does not appear on closer examination to be always the case, and sometimes as much harm as good may result, or what is more probable, neither much advantage, nor disadvantage, is the outcome. This can, of course, only be determined in each particular case from a knowledge of the whole life of a species and of the enemies that are likely to injure it.

Hudson has recorded ${ }^{1}$ a number of cases of this death1 "The Naturalist in La Plata." 
feigning instinct in higher animals, and attributes it to violent emotion, or fear, that produces a sort of swoon. He describes the gaucho boys' method, in La Plata, of catching the silverbill by throwing a stick or a stone at it, and then rushing toward the bird, "when it sits perfectly still, disabled by fear, and allows itself to be taken." He also states that one of the foxes (Canis a ware) and one of the opossums (Didilfly's asare " are strangely subject to the death-simulating swoon."

Hudson remarks that it seems strange that animals so well prepared to defend themselves should possess this "safeguard." When caught or run down by $\log s$, the fox fights savagely at first, but after a time its efforts stop, it relaxes, and it drops to the ground. The animal appears dead, and Iudson states that the dogs are "constantly taken in by it." He has seen the gatchos try the most barbarous tricks on a captive fox in this condition, and, despite the mutilations to which it was subjected, it did not wince. If, however, the observer draws a little away from the animal, "a slight opening of the eye may be detected, and finally, when left to himself, he does not recover and start up like an animal that has been stunned, but cautiously raises his head at first and only gets up when his foes are at a safe distance." Hudson, coming once suddenly upon a young fox, saw it swoon at his approach, and although it was lashed with a whip it did not move.

The common partridge of the pampas of La Plata (Hothura maculosa) shows this death-feigning instinct in a very marked degree. "When captured, after a few violent struggles to escape, it drops its head, gasps two or three times, and to all appearance dies." But if it is released it is off in an instant. The animal is excessively timid, and if frightened, may actually die simply from terror. If they are chased, and can find no thicket or burrow into which to escape, "they actually drop down dead on the plain. Probably when they feign death in their captor's hand they are in reality very near to death." 
In this latter instance it must appear very improbable that we are dealing with an instinct that has been built up by slow degrees on account of the benefit accruing at each stage to the individual. In fact, it appears that the instinct is in this case of really no use at all to the animal, for there can scarcely be any question of an escape by this action. Yet so far as we can judge it is the same instinct shown by other animals, and it is not logical to account for its origin in one case on the grounds of its usefulness, when we cannot apply the explanation in the other cases. If this be admitted, we have another illustration of the importance of keeping apart the origin of an instinct or of a structure and the fact of its usefulness or non-usefulness to the organism. Thus under certain conditions this death-feigning instinct might really be of use to the animal, while under other conditions and in other animals it may be of no advantage at all, and in still other conditions it may be a positive injury to its possessor. Perhaps we need not go outside of our own experience to find a parallel case, for the state of fright into which imminent danger may throw an individual may deprive him for the moment of the proper use of those very mental qualities of which he stands in this crisis in greatest need.

The peculiar behavior of cattle caused by the smell of blood is another case of an instinct whose usefulness to its possessors is far from apparent. It is known that cattle and horses and several wild animals become violently excited by the smell of blood. Hudson gives a vivid account of a scene witnessed by himself, the animals congregating, "and moving around in a dense mass, bellowing continually." Those animals that forced their way into the centre of the mass where the blood was "pawed the earth and dug it up with their horns, and trampled each other down in their frantic excitement."

This action leads us to a consideration of the behavior of animals toward companions in distress. "Herbivorous 
animals at such times will trample and gore the distressed one to death. In the case of wolves and other savagetempered carnivorous species the distressed fellow is frequently torn to pieces and devoured on the spot." If any one will be bold enough to claim in this case that this habit has been acquired because of advantage to the pack, i.c. if it be imagined that the pack gains more by feeding on a weak member than by letting him take his chances of recovery, it may be pointed out in reply that cattle also destroy their weak or injured, but do not devour them, and the same statement holds for birds, where the same instinct has often been observed. Romanes has suggested that the instinct of destroying the weak or injured members is of use because such members are a source of danger to the rest of the herd; but Hudson points out that it is not so much the weak and sickly nembers of the herd that are attacked in this way, as those that are injured, and concludes, "the instinct is not only useless, but actually detrimental." He suggests that these "wild abnormal movements of social animals" are a sort of aberration, so "that in turning against a distressed fellow they oppose themselves to the law of being." Yet whether we gain anything by calling this action aberrant or abnormal, the important fact remains that it is a definite response under certain external conditions, and is shown by all the individuals of the species.

The preceding illustrations of reactions that go to make up the so-called instincts of animals may be separated into those that are essential to the life of the individual or of the race, those that are of some apparent use, although not absolutely essential, and a few of no use at all, and fewer still that appear to be even injurious. If the latter reactions take place only rarely, as appears often to be the case, they are not sufficiently harmful to cause the destruction of the race. The evidence points to the conclusion, I believe, that the origin of these tropisms and instincts cannot be 
accounted for on the ground of their benefit to the individual or to the race; and it does not seem reasonable to make up one explanation for the origin of those that are essential, and another for those that are of little use or even of no use at all.

From what has been already said more than once, while discussing each particular case, the simplest course appears to be in all instances to look upon these instincts as having appeared independently of the use to which they may be put, and not as having been built up by selection of the individual variations that happen to give an organism some advantage over its fellows in a life and death struggle. It appears reasonable to deal with the origin of tropisms and instincts in general in the same way as in dealing with structures; for, after all, the tropism is only the outcome of some material or structural basis in the organism.

No attempt has been made here to interpret the more complex reactions of the nervous system, for until we can get some insight into the meaning of the simpler processes, we are on safer ground in dealing with these first. 


\section{CHAPTER XII \\ SEX AS AN ADAPTATION}

In what sense may the separation of all the individuals of a species into two kinds of individuals, male and female, be called an adaptation? Does any advantage result to the species that would not come from a non-sexual method of reproduction? Many attempts have been made to answer these questions, but with what success I shall now try to show.

There are four principal questions that must be considered :-

I. The different kinds of sexual individuals in the animal and plant kingdoms.

II. The historical question as to the evolution of separate sexes.

III. The factors that determine the sex in each individual developing from an eggr.

IV. The question as to whether any advantage is gained by having each new individual produced by the union of two germ-cells, or by having the germ-cells carried by two kinds of individuals.

While our main problem is concerned with the last of these topies, yet there would be little hope of giving a complete answer to it unless we could get some answer to the first three questions.

\section{The Different Kinds of Sexual Indmiduals}

Amongst the unicellular animals and plants the fusion of two (or more) individuals into a single one is generally regarded as the simplest, and possibly also the most primitive, 
method of sexual reproduction. Two amcebas, or amœbalike bodies, thus flow together, as it were, to produce a new individual.

In the more highly specialized unicellular animals, the processes are different. Thus in vorticella, a small, active individual unites with a larger fixed individual. The protoplasm fuses into a common mass, and a very complicated series of changes is passed through by the nucleus. In paramœcium, a free-swimming form very much like vorticella, two individuals that are alike unite only temporarily, and after an interchange of nuclear material they separate.

In the lower plants, and more especially in some of the simple aggregates or colonial forms, there are found a number of stages between species in which the uniting inclividuals are alike, and those in which they are clifferent. There are several species whose individuals appear to be exactly alike; and other species in which the only apparent difference between the individuals that fuse together is one of size; and still other species in which there are larger resting or passive individuals, and smaller active individuals that unite with the larger ones. In several of the higher groups, including the green algæe and seaweeds, we find similar series, which give evidence of having arisen independently of each other. If we are really justified in arranging the members of these groups in series, beginning with the simpler cases and ending with those showing a complete differentiation into two kinds of germ-cells, we seem to get some light as to the way in which the change has come about. It should not be forgotten, however, that it does not follow because we can arrange such a series without any large gaps in its continuity, that the more complex conditions have been gradually formed in exactly this way from the simplest conditions.

So far we have spoken mainly of those cases in which the forms are unicellular, or of many-celled species in which all the cells of the individual resolve themselves into one or the 
other kind of germ-cells. This occurs, however, only in the lowest forms. A step higher we find that only a part of the cells of the colony are set aside for purposes of reproduction. The cells surrounding these germ-cells may form distinct organs, which may show certain differences according to whether they contain male or female germ-cells. When these two kinds of cells are produced by two separate individuals, the individuals themselves may be different in other parts of the body, as well as in the reproductive organs.

When this condition is reached, we have individuals that we call males and females, because, although they do not themsclves unite to form new individuals, they produce one or the other kind of germ-cell. It is the germ-cells alone that now combine to form the new individual.

Amongst living groups of animals we find no such complete series of forms as exist in plants, and the transition from the one-celled to the many-celled forms is also more abrupt. On the other hand, we find an astonishing variety of ways in which the reproduction is accomplished, and several ways in which the germ-cells are carried by the sexual individuals. Let us examine some of the more typical conditions under the following headings: (I) sexes separate; (2) sexes united in the same individual; (3) parthenogenetic forms; (4) exceptional methods of propagation.

I. Seres Siparate; Ciniscrual Forms. ${ }^{1}$ - Although the animals with which we are more familiar have the sexes separate, this is far from being universal amongst animals and plants; and, in fact, can scarcely be said to be even the rule. When the sexes are separate they may be externally alike, and this is especially true for those species that do not unite, but set free their eggs and spermatozoa in the water, as fish, frogrs, corals, starfish, jellyfish, and many other forms. In other animals there are sometimes other secondary differ-

"Ciefles and'Thompson's "The Evolution of Sex" has been freely used in the preparation of this part of this chapter. 
ences in the sexes besides those connected with the organs of reproduction. Such differences are found, as we have seen, in insects, in some spiders, crustaceans, and in many birds and mammals. In a few cases the difference between the sexes is very great, especially when the female is parasitic and the male free, as in some of the crustaceans. In some other cases the male is parasitic on the female. Thus in Bonellia the male is microscopic in size, being in length only one-hundredth part of the female. In Hydatina scuta the male is only about a third as large as the female. It has no digestive tract, and lives only a few days. In another rotifer the males are mere sacs enclosing the male reproductive organs.

2. Hcmaphroditic Forms. - There are many species of animals and plants in which each individual contains both the male and the female organs of reproduction, and there are whole groups in which only these hermaphroditic forms occur. Thus in the ctenophors the eggs develop along one side of each radial canal and spermatozoa along the other. The group of flatworms is almost exclusively hermaphroditic. The earthworms and the lecches have only these bisexual forms, and in the mollusks, while a few groups have separate sexes, yet certain groups of gasteropods and of bivalve forms are entirely hermaphroditic.

In the common garden snail, although there are two sets of sexual ducts closely united, yet from the same reproductive sac both eggs and sperm are produced. The barnacles and the ascidians are for the most part hermaphroditic forms. Many other examples might be cited, but these will suffice to show that it is by no means unusual in the animal kingdom for the same individual to produce both male and female germcells. However, one of the most striking facts in this connection is that self-fertilization seldom takes place, so that the result is the same in certain respects as though separate sexes existed. This point will come up later for further consideration. 
3. Parthenogenctic Reproduction. - It has long been known that, in some cases, eggs that are not fertilized will begin to develop and may even produce new individuals. Tichomiroff showed that by rubbing with a brush the unfertilized eggs of the silkworm moth, a larger percentage would produce caterpillars than if they were not rubbed. During the last few years it has been shown that the development of a non-fertilized egg may be started in a number of ways. Such, for example, as by certain solutions of salt or of sugar, by subjecting the eggs to cold, or by simply shaking them.

There are certain groups of animals in which the males appear only at regular (in others at irregular) intervals. In their absence the females produce eggs that develop without being fertilized, i.c. parthenogenetically. The following examples will serve to show some of the principal ways in which this "virgin reproduction" takes place. In the group of rotifers the males are generally smaller than the females and are usually also degenerate. In some species, although degenerate males are present, they are unnecessary, since parthenogenesis is the rule. In still other species no males exist and the eggs develop, therefore, without being fertilized. In some of the lower crustaceans parthenogenesis occurs in varying degrees. In Apus males may be entirely absent at times in certain localities, and at other times a few, or even very many, males may appear. Some species of ostracod crustaceans seem to be purely parthenogenetic; others reproduce by means of fertilized eggs; and others by an alternation of the two processes. The crustaceans of the genus Daphnia produce two kinds of eggs. The summer eggs are small, and have a thin shell. These eggs develop without being fertilized, but in the autumn both male and female individuals develop from these unfertilized eggs, and the eggs of the female, the so-called winter eggs, are fertilizerl. These are also larger than the summer eggs, have thicker 
shells, and are much more resistant to unfavorable conditions. They give rise in the following spring to females only, and these are the parthenogenetic individuals that continue to produce during the summer new parthenogenetic eggs.

It is within the group of insects that some of the most remarkable cases of parthenogenesis that we know are found. In the moth, Psyche helix, only females are present, as a rule, but rarely males have been found. In another moth, Solcnobia trinquatrella, the female reproduces by parthenogenesis, but at times males appear and may then be even more numerous than the females. In the gall-wasps parthenogenetic generations may alternate with a sexual generation, and it is interesting to note that the sexual and the parthenogenetic generations are so different that they were supposed to belong to separate species, until it was found that they were only alternate generations of the same species.

The aphids or plant-lice reproduce during the summer by parthenogenesis, but in the autumn winged males and females appear, and fertilized winter eggs are produced. From these eggs there develop, in the following spring, the wingless parthenogenetic summer forms, which produce the successive generations of the wingless forms. As many as fourteen summer broods may be produced. By keeping the aphids in a warm temperature and supplying them with plenty of moist food, it has been possible to continue the parthenogenetic reproduction of the wingless forms for years. As many as fifty successive broods have been produced in this way. It has not been entirely determined whether it is the temperature or a change in the amount, or kind, of food that causes the appearance of the winged males and females, although it seems fairly certain that diminution in the food, or in the amount of water contained in it, is the chief cause of the change. 
In the honey-bee the remarkable fact has been well established that fertilized eggs give rise only to females (queens and workers), while unfertilized eggs develop into males. Whether a fertilized egg becomes a queen or a worker (sterile female) depends solely on the kind of food that is given to the young larva, and this is determined, in a sense, entirely by the bees themselves.

In plants also there are many cases of parthenogenesis known. Some species of Chara when kept under certain conditions produce only female organs, and seem to produce new plants parthenogenetically. In this case it appears that the same conditions that caused the plants to produce only female organs may also lead to the derelopment of the egg-cells without fertilization. In fact it is only by a combination of this kind that parthenogenesis could arise. The result is similar when the eggs of insects produce only females whose eggs are capable of parthenogenetic development. If a case should arise in which only females appeared whose eggs did not possess the power of parthenogenetic development, the species would die out.

In the green alga, Spirogyra, it has been found that if conjugation of two cells is prevented, a single cell may become a parthenogenetic cell. In a number of parasitic fungi the male organs appear to be degencrate, and from the female organs parthenogenetic development takes place. A small number of flowering plants are also capable of parthenogenetic reproduction.

There is a peculiarity in the development of the parthenogenetic egrss of animals that will be more fully discussed later, but may be mentioned here. Ordinarily an egg that becomes fertilized gives off two polar bodies, but in a number of cases in which parthenogenetic development occurs it has been found that only one polar body is given off. It is supposed that in such cases one polar body is retained, and that it plays the same part as the entrance of the spermatozoon of the male. 
4. Exceptional Cases. - Occasionally in a species that is unisexual an individual is found that is bisexual. The male of the toad, Pclobates fuscus, has frequently a rudimentary ovary in front of the testis. The same thing has been found in several species of fish. In Serranus, a testis is present in the wall of the ovary, and the eggs are said to be fertilized by the spermatozoa of the same individual. In frogs it has been occasionally found that ovary and testis may be associated in the same individual, or a testis may be present on one side, and a testis with an anterior ovarian portion on the other. Cases like these lead up to those in which the body itself may also show a mosaic of sex-characters, and it is noticeable that when this occurs there is nearly always a change in the reproductive organs also. Thus butterflies have been found with the wings and the body of one side colored like the male and the other side like the female. Similar cases have also been found in bees and ants. Bees have been found with the anterior part of the body of one sex and posterior part of another!

The preceding cases illustrate, in different ways, the fact that in the same individual both kinds of reproductive organs may suddenly appear, although it is the rule in such species that only one set develops. Conversely, there are cases known, especially amongst plants, in which individuals, that usually produce male and female organs (or more strictly spores of two kinds from which these organs develop), produce under special conditions only one or the other kind. Facts like these have led to the belief that each individual is potentially bisexual, but in all unisexual forms one sex predominates, and the other remains latent. This idea has been the starting-point for nearly all modern theories of sex.

An excellent illustration of this theory is found in those cases in which the same individual may be male at one time and female at another. For instance, it is said that in one of the species of starfish (Astcrina gibbosa) the individuals at Roscoff are males for one or two years, and then become 
females. At Banyuls they are males for the first two or three years, and then become females; while at Naples some are always males, others females, sone hermaphrodites, others transitional as in the cases just given. In one of the isopod crustaceans, Angiostomum, the young individuals are males and the older females. In Mysostomum glabrum the young animal is at first hermaphroditic, then there is a functional male condition, followed by a hermaphroditic condition, and finally a functional female phase, during which the male reproductive organs disappear.

The flowers of most of the flowering plants have both stamens and pistils, which contain the two kinds of spores out of which the male and female germ-cells are formed. The stamens become mature before the pistils, as a rule, but in some cases the reverse is the case. This difference in the time of ripening of the two organs is often spoken of as an adaptation which prevents self-fertilization. The latter is supposed to be less advantageous than cross-fertilization. This question will be more fully considered later.

Before we come to an examination of the question of the adaptations involved in the cases in which the sexes are separate, and the different times at which the sex-cells are ripened, it will be profitable first to examine the question as to what determines in the egg or young whether a male or a female or a hermaphroditic form shall arise.

\section{The Deternination of Sex}

A large number of riews have been advanced as to what determines whether an exg will give rise to a male or to a female indiridual. The central question is whether the fertilized egg has its sex already determined, or whether it is indifferent; and if the latter, what external factor or factors determine the sex of the embryo. I,et us first examine the view that some external factor determines the sex of the individual, and 
then the evidence pointing in the opposite direction. Among the different causes suggested as determining the sex of the embryo, that of the condition of the egg itself at the time of fertilization has been imagined to be an important factor in the result. Another similar view holds that the condition of the spermatozoon plays the same rôle. For instance, it has been suggested that if the egg is fertilized soon after it leaves the ovary, it produces a female, but if the fertilization is delayed, a male is produced. It has also been suggested that the relative age of the male and the female parents produces an effect in determining the sex of the young. There is no satisfactory evidence, however, showing that this is really the case.

Another view suggested is that the sex is determined by the more vigorous parent; but again there is no proof that this is the case, and it would be a difficult point to establish, since as Geddes and Thompson point out, what is meant by greater vigor is capable of many interpretations. Somewhat similar is the idea that if the conditions are favorable, the embryo develops further, as it were, and becomes a male; but there are several facts indicating that this view is untenable.

Düsing maintains that several of these factors may play a part in determining the sex of the embryo, and if this be true, the problem becomes a very complex one. He also suggests that there are self-regulative influences of such a kind that, when one sex becomes less numerous, the conditions imposed in consequence on the other sex are such as to bring the number back to the normal condition; but this idea is far from being established. The fact that in some species there are generally more individuals of one sex than of the other shows that this balance is not equally adjusted in such forms.

Of far greater value than these speculations as to the origin of sex are the experiments that appear to show that nutrition is an important factor in determining sex. Some of the 
earlier experiments in this direction are those of Born and of Yung. By feeding one set of tadpoles with beef, Yung found the percentage of females that developed to be greatly increased, and a similar increase was observed when the tadpoles were fed on the flesh of fish. An even greater effect was produced by using the flesh of frogs, the percentage rising to 92 females in every hundred. These results have been given a different interpretation by Pflüger and by others, and, as will be pointed out later, there is a possible source of error that may invalidate them.

Somewhat similar results have been obtained by Nussbaum for one of the rotifers. He found that if the rotifer is abundantly fed in early life, it produces fomale cogss, that is, larger eggs that become females; while if sparingly fed, it produces only small eggs, from which males develop. It has been claimed also in mammals, and even in man, that sex is to some extent determined by the nourishment of the individual.

Some experiments made by Mrs. Treat with caterpillars seemed to show that if the caterpillars were well nourished more female moths were produced, and if starved before pupation more males emerged. But Riley has pointed out that since the larger female caterpillars require more food they will starve sooner than the males, and, in consequence, it may appear that proportionately more male butterflies are born when the caterpillars are subjected to a starvation diet. This point of view is important in putting us on our guard against hastily supposing that food may directly determine sex. Unless the entire number of individuals present at the beginning of the experiment is taken into account, the results may be misleading, because the conditions may be more fatal to one sex than to the other.

In some of the hymenopterous insects, the bees for example, it has been discovered that the sex of the embryo is determined by the entrance, or lack of entrance, of the spermatozoon. In the honey-bee all the fertilized eggs produce 
females and the unfertilized eggs males. The same relation is probably true also in the case of ants and of wasps. In the saw-flies, the conditions are very remarkable. Sharp gives the following account of some of these forms: ${ }^{1}$ - "It is a rule in this family that males are very much less numerous than females, and there are some species in which no males have been discovered. This would not be of itself evidence of the occurrence of parthenogenesis, but this has been placed beyond doubt by taking females bred in confinement, obtaining unfertilized eggs from them, and rearing the larva produced from the eggs. This has been done by numerous observers with curious results. In many cases the parthenogenetic progeny, or a portion of it, dies without attaining full maturity. This may or may not be due to constitutional weakness, arising from the parthenogenetic state. Cameron, who has made extensive observations on this subject, thinks that the parthenogenesis does involve constitutional weakness, fewer of the parthenogenetic young reaching maturity. This, he suggests, may be compensated for when the parthenogenetic progeny is all of the female sex by the fact that all those that grow up are producers of eggs. In many cases the parthenogenetic young of Tenthredinidre are of the male sex, and sometimes the abnormal progeny is of both sexes. In the case of one species - the common currant-fly, Ncmatus ribesii - the parthenogenetic progeny is nearly, but not quite always, entirely of the male sex; this has been ascertained again and again, and it is impossible to suggest in these cases any advantage to the species to compensate for constitutional parthenogenetic weakness. On the whole, it appears most probable that the parthenogenesis, and the special sex produced by it, whether male or female, are due to physiological conditions of which we know little, and that the species continues in spite of the parthenogenesis rather than profits by it. It is worthy of remark that one of 1 “The Cambridge Natural History," Vol. V, “Insects," by David Sharp. 
the species in which parthenogenesis with the production of males occurs - Nematus ribesii - is perhaps the most abundant of saw-flies."

It has been pointed out that in a number of species of animals and plants only parthenogenetic females are present at certain times. In a sense this means a preponderance of one sex, but since the eggs are adapted only to this kind of development, it may be claimed that the conditions in such cases are somewhat different from those in which eggs that would be normally fertilized may develop in the absence of fertilization. Nevertheless, it is generally supposed that the actual state of affairs is about the same. It is usually assumed, and no doubt with much probability, that these parthenogenetic forms have evolved from a group which originally had both male and female forms. One of the most striking facts in this connection is that in the groups to which these parthenogenetic species belong there are, as a rule, other species with occasional parthenogenesis, and in some of these the males are also fewer in number than the females.

In the aphids, the parthenogenetic eggs give rise during the summer to parthenogenetic females, but in the autumn the parthenogenetic eggs give rise without fertilization both to males and to females. It appears, therefore, that we can form no general rule as to a relation between fertilization and the determination of sex. While in certain cases, as in the bees, there appears to be a direct connection between these two, in other cases, as in that of the aphids just mentioned, there is no such relation apparent.

Geddes and Thompson have advocated a view in regard to sex which at best can only serve as a sort of analogy under which the two forms of sex may be considered, rather than as a legitimate explanation of the phenomenon of sex. They rest their view on the idea that living material is continually breaking down and building up An animal in which there is an excess of the breaking-down process is a male, and 
one that is more constructive is a female. Furthermore, whichever process is in the excess during development determines the sex of the individual. Thus, if conditions are very favorable, there will be more females produced; but if, on the other hand, there is an excess of the breaking-down process, males are produced. So far, the process is conceived as a purely physiological one, but to this the authors then apply the selection hypothesis, which, they suppose, acts as a sort jef break or regulation of the physiological processes, or in other words as a directive agent. They state: "Yet the sexual dimorphism, in the main, and in detail, has an adaptive significance, also securing the advantages of cross-fertilization and the like, and is, therefore, to some extent the result of the continual action of natural selection, though this may, of course, check variation in one form as well as favor it in another." Disregarding this last addition, with which Geddes and Thompson think it necessary to burden their theory, let us return to the physiological side of the hypothesis. Their idea appears to me a sort of symbolism rather than a scientific attempt to explain sex. If their view had a real value, it ought to be possible to determine the sex of the developing organism with precision by regulating the conditions of its growth, and yet we cannot do this, nor do the authors make any claim of being able to do so. The hypothesis lacks the only support that can give it scientific standing, the proof of experiment.

There have been made, from time to time, a number of attempts to show that the sex of the embryo is predetermined in the egg, and is not determined later by external circumstances. In recent years this view has come more to the front, despite the apparent experimental evidence which seemed in one or two cases to point to the opposite view. One of the most complete analyses of the question is that of Cuénot, who has attempted to show that the sex of the embryo is determined in the egg, before or at the time of 
fertilization. He has also examined critically the evidence that appeared to show that external conditions, acting on the embryo, may determine the sex, and has pointed out some possible sources of error that had been overlooked. The best-known case is that of the tadpole of the frog, but Cuénot shows not only that there are chances of error in this experiment as carried out, but also, by his own experiments and observations, that the facts themselves are not above suspicion. He points out that at the age at which some of the tadpoles were when the examination was made, it was not always possible to tell definitely the sex of the individual, and least of all by means of the size alone of the reproductive organs, as was supposed, in one case at least, to be sufficient. In his own experiments he did not find an excess of one sex over the other as a result of feeding.

Cuénot points out that Brocadello found that the larger eggs laid by the silkworm give rise to from 88 to 95 per cent of females, and the small eggs to from 88 to 92 per cent of males. Joseph has confirmed this for Ocncria dispar, and Cuénot himself also reached this conclusion. Korschelt found that the large eggs of Dinophilus produced females and the small ones males. Cuénot experimented with three species of flies, and found that when the maggots were well nourished the number of the individuals of the two sexes was about equal, and when poorly nourished there were a few more females in two cases, and in another about the same number of males and females.

It has been claimed that the condition of nourishment of the mother may determine the number of eggs of a particular sex, but Cuénot found, in three species of flies which he raised, that there was a slight response in the opposite direction. He concludes that the condition of the mother is not a factor in the determination of sex.

The first egg of the two laid in each set by the pigeon is said, as a rule, to produce a male, and the second a female. 
Both Flourens and Cuénot found this to be the case in the few instances that they examined, but Cuénot has shown that this does not always happen. Even when this occurs, it has not been determined whether the result depends on something in the egg itself, that causes a male egg to be set free first, or on some external condition that determines that the first egg shall become a male. It has been claimed that the age of the spermatozoon might in this and in other cases determine the result; but Gerbe has shown that if the domestic hen is isolated for fifteen days after union with the male, she will continue to produce fertile eggs from which both sexes are produced, without showing any relation between the time the eggs are laid and the particular sex that develops.

Cuénot does not discuss whether sex is determined by the nucleus or by the protoplasm, but if, as he thinks probable, the size of the egg is a determining factor, it would appear that the protoplasm must be the chief agent. Even if this were the case it would still be possible that the size of the egg itself might be connected with some action on the part of the nucleus. If, as seems probable, identical twins come from halves of the same egg, then, since they are of the same sex, the absolute amount of protoplasm cannot be a factor in sex determination.

As a basis for the discussion that follows, certain processes that take place during the maturation divisions of the egg and of the spermatozoon must be briefly noticed. After the egg leaves the ovary it extrudes a minute body called the first polar body (Fig. $6 \mathrm{~B}, \mathrm{C}, \mathrm{D})$. This process of extrusion is really a cell division accompanied by the regular mitotic division of the nucleus; but since one of the products of the division, the polar body, is extremely small, the meaning of the process was not at first understood. The half of the nucleus, that remains in the egg, divides again, and one of its halves is thrown out into a second polar body (Fig. $6 \mathrm{E}$, F, G). Meanwhile, the first polar body has divided into two 
equal parts, so that we find now three polar bodies and the egg (Fig. $6 \mathrm{G}$ ). A strictly analogous process takes place in the formation of the spermatozoa (Fig. $7 \mathrm{~B}-\mathrm{F}$ ). The mother-cell of the spermatozoon divides into two parts, which are equal in this case (Fig. $7 \mathrm{~B}-\mathrm{D}$ ). Each of these then

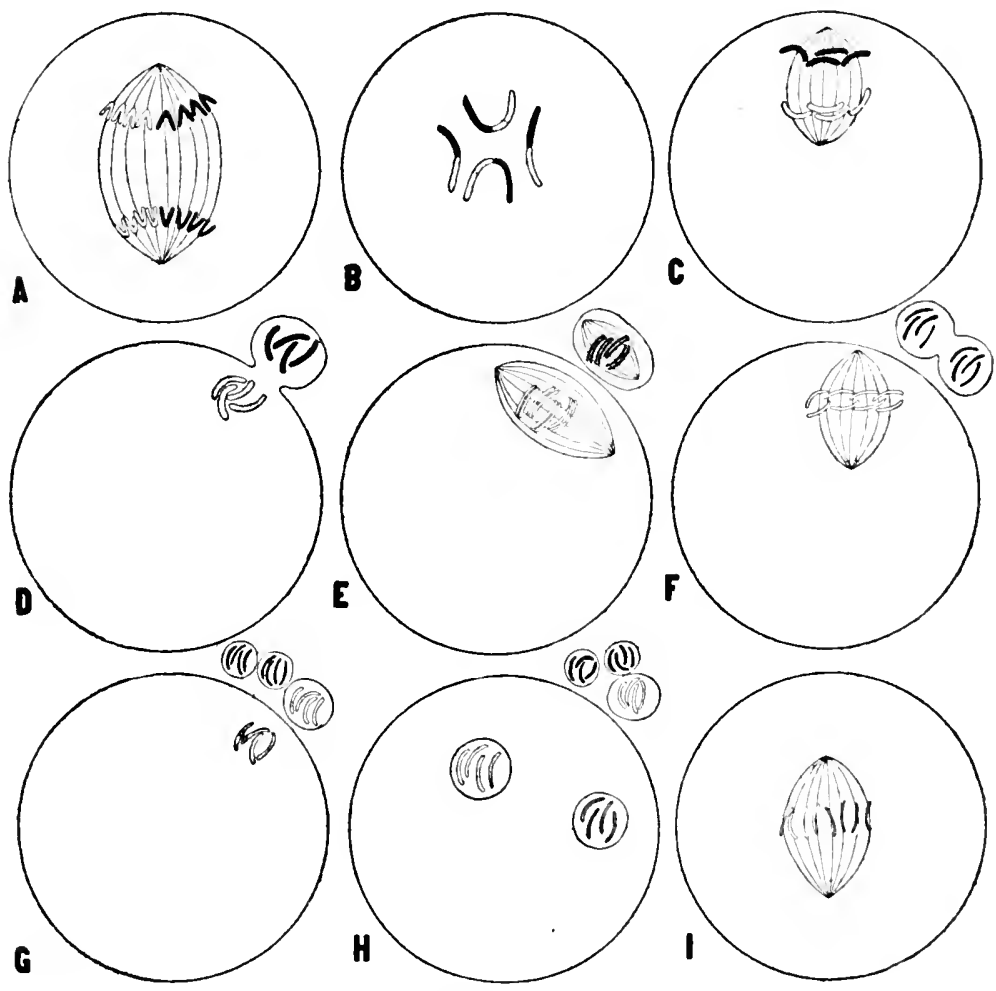

FIG. 6. - I)iagram showing the maturation of the egg.

divides again (Fig. 7 F, F), producing four cells that are comparable to the three polar bodies and the mature egro. Each of the four becomes a functional spermatozoon (Fig. 7 G, H). Thus while in the maturation of the egg only the egr itself is capable of development, in the case of the male cells all four products of the two maturation divisions are functional. 
Now, in certain cases of parthenogenesis, it has been found that one of the polar bodies may not be given off, but, remaining in the egg, its nucleus reunites with the egg nucleus, and thus takes the place of the spermatozoon, which does exactly the same thing when it fertilizes the egg, i.c. the nucleus of
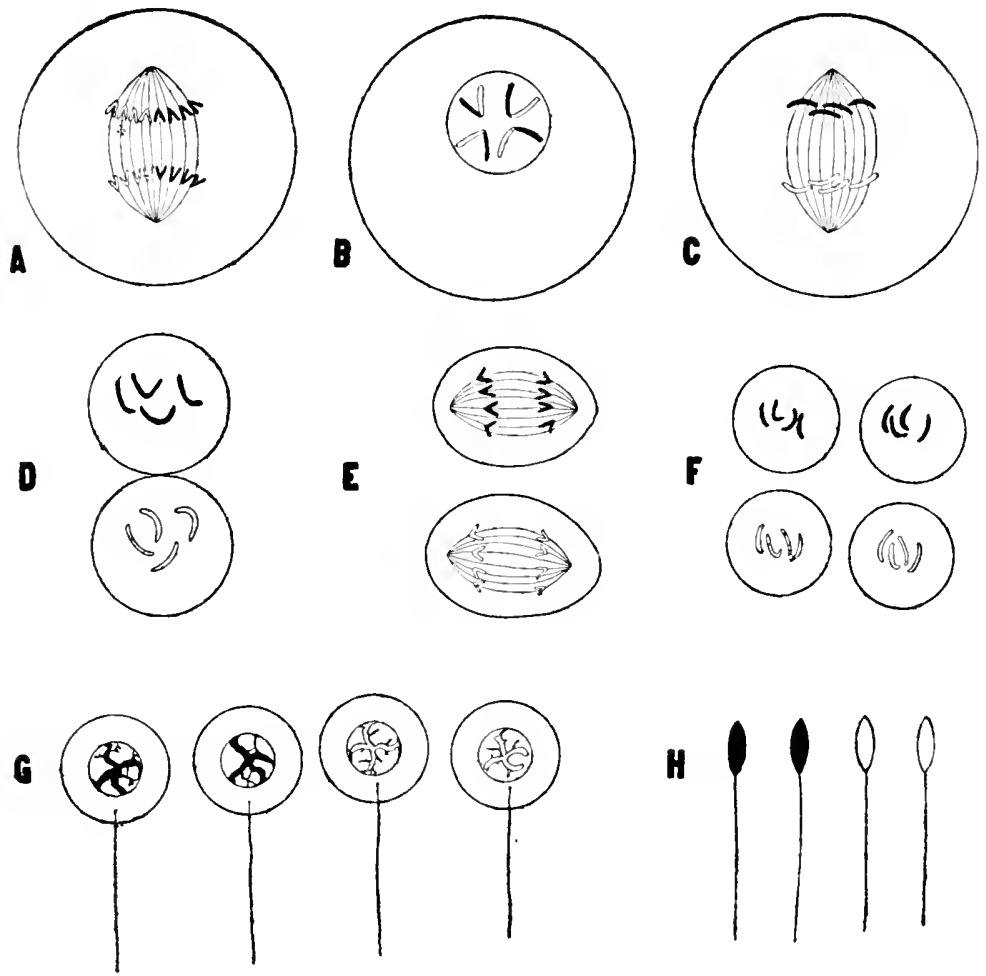

FIG. 7.-Diagram showing the maturation of the spermatozoon.

the spermatozoon unites with the nucleus of the egg. This fact in regard to the action of the polar body in fertilization is not as surprising as appears at first sight, for if each of the polar bodies is equivalent to a spermatozoon, the fertilization of the egg by one of its own polar bodies conforms to theory.

There is a considerable body of evidence showing that in many eggs at one of the two maturation divisions the 
chromatin rods derived from the nucleus are divided crosswise (Fig. $6 \mathrm{~B}, \mathrm{C}$ ). The same thing occurs at one of the two divisions in the formation of the spermatozoon (Fig. $7 \mathrm{~B}, \mathrm{C}$ ). At the other division to form the other polar body (or the other sperm-cell) the chromatin rods appear to be split lengthwise, as in ordinary cell division (Fig. $6 \mathrm{E}, \mathrm{F}, \mathrm{G}$ ). In recent years the cross-ditision of the chromatin rods has attracted a great deal of notice, and Weismann in particular drew attention to the possible importance of this kind of division.

There is another fact that gives this division especial significance. It has been discovered that the number of chromosomes that appears in each dividing cell of the organism is a constant number, but it has also been discovered that the egg, before extruding its polar bodies, and the mothercell of the spermatozoon (Figs. 6, 7 B), contain exactly half of the number of chromosomes that are characteristic of the body-cells of the same animal (Figs. 6, 7 A). Now there is good evidence to show that the reduction in number is due to the chromosomes uniting sometimes end to end in pairs, as shown in Figures $A$ and $B$. Furthermore, it has been suggested that at one of the maturation divisions, when the chromosomes divide crosswise, the united chromosomes are separated (Figs. 6, 7 B, C), so that one remains in the egg and the other goes out into the polar body. The same thing is supposed to occur at one of the maturation divisions of the sperm mother-cell. A further consicleration of capital importance in this connection has been advocated by Montgomery and by Sutton, namely, that, when the chromosomes unite in pairs, a chromosome from one parent unites with one from the other parent. Consequently at one of the two reduction divisions maternal and paternal chromosomes may separate again, some to go to one cell, some to the other.

When the spermatozoon enters the egg it brings into the egg as many new chromosomes as the egg itself possesses at 
this time, and the two nuclei, uniting into a single one, furnish the total number of chromosomes characteristic of the animal that develops from the egg. At first the chromosomes that are brought in by the spermatozoon lie at one side of the fused nucleus, and those from the egg itself at the other side. This arrangement appears, however, in some cases at least, to be lost later. At every division of the nucleus, each chromosome divides and sends a half to each of the daughter-nuclei. Thus every cell in the body contains as many paternal as maternal chromosomes. This statement also applies to the first cells that go into the reproductive organs, some of which become the mother-cells of the germcells. Later, however, in the history of the germ-cells, just before the maturation divisions, - these chromosomes are supposed to unite in pairs, end to end, as explained above, to give the reduced number. Later there follows the separation of these paired chromosomes at one of the two maturation divisions. If at this time all the paternal chromosomes should pass to one pole, and all the maternal to the other, the germ-cell ceases to be mixed, and becomes purely paternal or maternal. If this ever occurs, the problem of heredity may become simplified, and even the question of sex may be indirectly involved; but it has not been established that, when the reduced number of chromosomes is formed, there is a strict union between the paternal and maternal chromosomes, and if not, the subsequent separation is probably not along these lines. If, however, the chromosomes contain different qualities, as Boveri believes, there may be two kinds of eggs, and two kinds of spermatozoa in regard to cach particular character. It is this last assumption only that is made in Mendel's theory of the purity of the germ-cells.

Several attempts have been made at different times to connect the facts in regard to the extrusion of the polar bodies with those involved in the determination of sex. Minot suggested, in I 877 , that the egg ejects by means of the 
polar bodies its male elements, which are again received in the fertilization of the egg by the spermatozoon. The same idea has also been expressed by others. It has been objected to this view that one polar body ought to suffice, and that no similar throwing out of part of its substance is found in the process of formation of the spermatozoon, which should, on the hypothesis, throw out its female elements. It would seem, on first thought, that this view might find support in the idea expressed above, namely, that in one of the polar bodies half of the chromosomes pass out, so that there is conceivably a separation of the maternal from the paternal. If this were the case also in the spermatozoa, then two of each four would be paternal and two maternal. This is, however, a very different thing from supposing them to be male and female, for it by no means follows, because the chromosomes correspond to those of the father or of the mother in the sum of their characters, that they are, therefore, also male or female in regard to sex.

It has been pointed out already, that in most parthenogenetic egoss only one polar body is extruded. There are, it is true, a few apparent exceptions to this rule, but in most cases it is certain that only one is extruded. In several cases the beginning of the formation of the second maturation division of the nucleus takes place, but after the chromosomes have divided they come together again in the nucleus. If each polar body be interpreted as equivalent to a spermatozoon, then this result is rather a process of self-fertilization than true parthenogenesis. It is, nevertheless, true that in some cases development seems to go on after both polar bodies have been extruded. Moreover, it has been found possible to cause the eggs of the sea-urchin to bersin their development by artificial solutions after they have extruded both polar bodies. A single spermatozoon may also produce an embryo if it enters a piece of egg-protoplasm without a nueleus. The last instance is a case of 
male parthenogenesis, and if the theory of the equivalency of spermatozoon and egg be correct, this is what should occur.

Quite recently, Cuénot, Beard, Castle, and Lenhossek have contended that the differentiation of sex is the outcome of internal factors. They think that the view that sex is determined by external agents is fundamentally erroneous. The fallacies that have given rise to this conception, Castle points out, are, first, that in animals that reproduce sometimes by parthenogenesis and sometimes by fertilized eggs, the former process is favored by good nutrition and the latter by poor nutrition. This only means, in reality, Castle thinks, that parthenogenetic reproduction is favored by external conditions, and this kind of reproduction, he thinks, is a thing sui gencris, and not to be compared to the formation of more females in the sexual forms of reproduction. There is no proof, however, that this is anything more than a superficial distinction, and it ignores the fact that in ordinary cases the females sometimes lay parthenogenetic eggs which differ, as far as we can see, from eggs that are destined to be fertilized in no important respect. More significant, it seems to me, is the fact that only parthenogenetic females develop the following spring from the fertilized eggs of the last generation of the autumn series, whose origin is described to be due to lack of food. We find, in the case of aphids, that unfertilized parthenogenetic eggs and also fertilized eggs give rise to females only, while a change in the amount of food causes the parthenogenetic eggs to give rise both to males and to females. This point is not, I think, fully met by Castle, for even if the change in food does not, as he claims, cause only one sex to appear, yet lack of food does seem to account for the appearance of the males at least.

The other fallacy, mentioned by Cuénot, is that the excess of males that has been observed when the food supply is limited is due to the early death of a larger percentage 
of females, which require more food, but this still fails to account for the excess of females when more food is given, provided Yung's experiments on tadpoles are correct. It may be, however, in the light of Pflüger's results, that there has been some mistake in the experiments themselves.

We may now proceed to examine Castle's argument, attempting to show in what way sex is predetermined in the embryo. His hypothesis rests on the three following premises: “( I) the idea of Darwin, that in animals and plants of either sex the characters of the opposite sex are latent; (2) the idea of Mendel, that in the formation of the gametes [germcells] of hybrids a segregation of the parental characters takes place, and when in fertilization different segregated characters meet, one will dominate, the other become latent or recessive; (3) the idea of Weismann, that in the maturation of egg and spermatozoon a segregation is attended by a visible reduction in the number of chromosomes in the germinal nuclei."

Expressed in a somewhat more general way, Castle suggests that each egg and each spermatozoon is either a male or a female germ-cell (and not a mixture of the two), and when a female egg is fertilized by a male spermatozoon, or itice a'ersu, the individual is a sexual hybrid with one sex dominating and the other latent. The assumption that there are two kinds of eggs, male and female, and two kinds of spermatozoa, male and female, is not supported by any direct or experimental evidence. Moreover, in order to carry out the hypothesis, it is necessary to make the further assumption that a female egg can only be fertilized by a male spermatozoon, and a male egg by a female spermatozoon. While such a view is contrary to all our previous ideas, yet it must be admitted that there are no facts which disprove directly that such a selection on the part of the germ-cells takes place. If these two suppositions be granted, then Castle's hypothesis is as follows:- 
In order that half of the individuals shall become males and half females it is necessary to assume that in some individuals the male element dominates and in others the female, and since each fertilized egg contains both male and female elements, it is necessary to assume that either the egg or the spermatozoon contains the dominating element.

Castle supposes that in hermaphroditic organisms the two characters "exist in the balanced relationship in which they were received from the parents," but, as has just been stated, in unisexual forms one or the other sex dominates, except of course in those rare cases, as in the bees and ants, where half of the body may bear the characters of one sex, and the other half that of the other sex.

In parthenogenetic species the female character is supposed to be uniformly stronger, so that it dominates in every contest, "for the fertilized egg in such species develops invariably into a female." Under certain circumstances, as Castle points out, the parthenogenetic female produces both males and females, and this is also true in the occasional development of the unfertilized egg of the silkworm moth, and of the gypsy moth, in which both male and female individuals are produced by parthenogenesis. These facts show that even in unfertilized eggs both sexes are potentially present; but this might be interpreted to mean that some eggs are male and some female, rather than that each egg has the possibility of both kinds of development. If, however, one polar body is retained in these parthenogenetic eggs, then $e x$ hypothese each egg would contain the potentialities of both sexes (if the polar body were of the opposite sex character). It seems necessary to make this assumption because in some parthenogenetic forms males and females may be produced later by each individual, as in the aphids, and this could not occur if we assume that some parthenogenetic eggs are purely male and some female. 
Castle assumes, in fact, that in animals like daphnids and rotifers one polar body only is extruded, and the other (the second) is retained in the egg, and hence the potentiality of producing males is present. In the honey-bee, on the contrary, Castle assumes that both polar bodies are extruded in the unfertilized egg (and there are some observations that support this idea), and since only males are produced from these, he believes it is the female element that has been sent out into the second polar body. This hypothesis is necessary, because Castle assumes that when both elements are present in the bee's eggs, the female element dominates. "Hence, if the egg which has formed two polar cells develops without fertilization, it must develop into a male. But if such an egg is fertilized, it invariably forms a parthenogenetic female $q\left(o^{*}\right)$, that is, an individual in which the male character is recessive. Accordingly the functional spermatozoon must in such cases invariably bear the female charactir, and this is invariably dominant over the male character when the two meet in fertilization."

If it should prove generally true that the size of the eggis one of the factors determining the sex, we have still the further question to consider as to whether some eggs are bigger because they are already female, or whether all eggs that go beyond a certain size are females, and all those that fail to reach this are males. If this is the case, an animal might produce more females if the external conditions were favorable to the growth of the eggs, and if in some cases these large eggs were capable of dereloping, parthenogenetic races might become established. Should, however, the conditions for nutrition become less farorable, some of the eggs might fall below the former size and produce males. It is not apparent, however, why all the fertilized autumu eggs of the aphids should give rise to females, for although these eggs are known to be larger than the summer eggs, yet they are produced under unfavorable conditions. 
The preceding discussion will show how far we still are from knowing what factors determine sex. Castle's argument well illustrates how many assumptions must be made in order to make possible the view that sex is a predetermined quality of each germ-cell. Even if these assumptions were admissible, we still return to the old idea that the fir tilized $\operatorname{gg} g$ has both possibilities, and something determines which shall dominate. Until we have ascertained definitely by experimental work whether the sex in some forms can be determined by external conditions, it is almost worthless to speculate further. Whatever decision is reached, the conclusion will have an immediate bearing on the question to be next discussed. Meanwhile, we can at least examine some of the theories that have been advanced as to what advantage, if any, has been gained by having the individuals of many classes divided into two kinds, male and female.

\section{Sex as a Phenomenon of Adaptation}

Of what advantage is it to have the individuals of many species separated into males and females? It is obviously a disadvantage from the point of view of propagation to have half of the individuals incapable of producing young, and the other half also incapable of doing so, as a rule, unless the eggs are fertilized by the other sex. Is there any compensation gained because each new individual arises from two parents instead of from one? Many answers have been attempted to these questions.

At the outset it should be recognized that we are by no means forced to assume, as is so often done, that because there is this separation of the sexes it must have arisen on account of its advantage to the species. Whether the result may be of some benefit regardless of how it arose, may be an entirely different question. It would be extremely difficult to weigh the relative advantages (if there are any) 
and disadvantages (that are obvious as pointed out above), nor is it probable that in this way we can hope to get a final answer to our problem. We may begin by examining some of the modern hypotheses that have been advanced in this connection.

Darwin has brought together a large number of facts which appear to show the beneficial effects of the union of germ-cells from two different individuals. Conversely, it is very generally believed, both by breeders and by some experimenters, that self-fertilization in the case of hermaphroditic forms leads, in many cases, though apparently not in all, to the production of less vigorous offspring. Darwin's general position is that it is an advantage to the offspring to have been derived from two parents rather than to have come from the union of the germ-cells of the same individual, and he sees, in the manifold contrivances in hermaphroditic animals and plants to insure cross-fertilization, an adaptation for this purpose.

This question of whether self-fertilization is less advantageous than cross-fertilization is, however, a different question from that of whether non-scrual methods of reproduction are less advantageous than sexual ones. Since some plants, like the banana, have been propagated for a very long time solely by non-sexual methods without any obrious detriment to them, it is at first sight not easy to see what other advantage could be gained by the sexual method. The case of the banana shows that some forms do not require a sexual method of propagation. Other forms, however, are so constituted, as we find them, that they cannot reproduce at the present time except by the sexual method. In other words, the latter are now adapted, as it were, to the sexual method, and there is no longer any choice between the two methods. The question of whether a non-sexual form might do better if it had another method of propagation is not, perhaps, a protitable question to discuss. 


\section{Sex as an Adaptation}

What we really need to know is whether or not the sexual method was once acquired, because it was an advantage to a particular organism, or to the species to reproduce in this way. It is assumed by many writers that this was the case, but whether they have sufficient ground for forming such an opinion is our chief concern here. On the other hand, it is conceivable, at least, that if the sexual method once became established, it might continue without respect to any superiority it gave over other methods, and might finally become a necessary condition for the propagation of particular species. Thus the method would become essential to propagation without respect to whether the species lost more than it gained. Whichever way the balance should turn, it might make little difference, so long as the species was still able to propagate itself.

Brooks made the interesting and ingenious suggestion that the separation of the sexes has been brought about as a sort of specialization of the individuals in two directions. The male cells are supposed to accumulate the newly acquired characters, and represent, therefore, the progressive element in evolution. The female cells are the conservative element, holding on to what has been gained in the past. It does not seem probable, in the light of more recent work, that this is the function of the two sexes, and it is unlikely that we could account for the origin of the two sexes through the supposed advantage that such a specialization might bring about. A number of writers, Galton, Van Beneden, Bütschli, Maupas, and others, have looked at the process of sexual reproduction as a sort of renewal of youth, or rejuvenescence of the individuals. There is certainly a good deal in the process to suggest that something of this sort takes place, although we must be on our guard against assuming that the rejuvenescence is anything more than the fulfilment of a necessary stage in the life history. Weismann has ridiculed this suggestion on the ground that it is inconceivable that 
two organisms, decrepid with old age, could renew their youth by uniting. Two spent rockets, he says, cannot be imagined to form a new one by combining. There is apparent soundness in this argument, if the implication is taken in a narrow physical sense. If, on the other hand, the egg is so constituted that at a certain stage in its development an outside change is required to introduce a new phase, then the conception of rejuvenescence does not appear in quite so absurd a light.

This hypothesis of rejuvenescence is based mainly on certain processes that take place in the life history of some of the unicellular animals. Let us now see what this evidence is. The results of certain experiments carried out by Maupas on some of the ciliate protozoans have been fruitful in arousing discussion as to the ultimate meaning of the sexual process. Maupas' experiments consisted in isolating single individuals, and in following the history of the descendants that were produced non-sexually by division. He found that the descendants of an individual kept on dividing, but showed no tendency to unite with each other. After a large number of generations had been passed through (in Stylonychia pustulata, between 128 and I75; in Leucophys fatula, 300 to 450 ; and in Onychodromus grandis, 140 to 230 generations), the division began to slow down, and finally came to a standstill. Maupas found that if he took one of these run-down individuals, and placed it with another in the same condition from another culture, that had had a different parentage, the two would unite and the so-called process of conjugation take place. This process consists for the species used, in the temporary union and partial fusion of the protoplasm of the two individuals, of an interchange of micronuclei, and of a fusion, in each individual, of the micronucleus received from the other individual with one of its own. The individuals then separate, and a new nucleus (or nuclei) is formed out of the fused pair. 
The individuals in question, in which this interchange of micronuclei has taken place, undergo a change, and behave differently from what they did before. They feed, become larger and less vacuolated, and are more active. They soon begin once more to divide. Maupas found that an individual that has conjugated will run through a new cycle of divisions, which will, however, after a time also slow down, unless conjugation with another individual having a different history takes place. If conjugation is prevented, the individual will die after a time. These results seemed to show that the division phase of the life history cannot go on indefinitely, and that through conjugation the individual is again brought back to the starting-point.

Quite recently Calkins has carried out a somewhat similar series of experiments, which have an important bearing on the interpretation of Maupas' results. The experiment of isolating an individual and tracing the career of its descendants was repeated with the following results: two series were started, the original forms coming from different localities. Of their eight descendants four of each were isolated. The remaining four of each set were kept together as stock material. The rate of division was taken as the measure of vitality. The animals divided more or less regularly from February to July. After each division (or sometimes after two divisions) the individuals were separated. About the 3oth of July the paramœcia began to die "at an alarming rate, indicating that a period of depression had apparently set in, or degeneration in Maupas' sense." $U_{p}$ to this time the animals had been living in hay infusion, renewed every few days, from which they obtained the bacteria on which they feed. Calkins tried the effect of putting the weakened paramœcia into a new environment. Infusion of vegetables gave no good results, but meat infusions proved successful. "The first experiment with the latter was with teased liver, which was added to the usual hay infusion. The result was 


\section{4}

very gratifying, for the organisms began immediately to grow and to divide, the rate of division rising from five to nine divisions in successive ten-day periods." This beneficial effect was not lasting, however, and after ten days the paramoecia began to die off faster than before, and the renewed application of the liver extract failed to revive them. A number of other extracts were then tried without effect. Finally they were transferred to the clear extract of lean beef in tap water. The effect of this medium was interesting, for, although it restored the weakened vitality, there was no rapid increase in the rate of division, as when first treated with the teased liver. The infusoria were, however, now large and vigorous, and did not die unless transferred from the beef medium to the usual hay infusion. "When this was attempted, they would become abnormally active and would finally die. The division rate gradually increased during the month of August until, in the last ten days, they averaged six generations. Finally, in September, the attempts to get them back on the old diet of hay infusion were successful, and then the division rate went up at once to twelve times in ten days, and a month later they were dividing at the rate of fifty times a month."

"These cultures went on well until December, when the paramoecia began to die again. They were saved once more with the beef extract, and when returned later to the hay infusion continued through another cycle of almost three months. Some of these were treated, once a week for twenty-four hours, with the beef extract, and while the two sets ran a parallel course at first, those kept continuously in the hay infusion died after a time, but those that had been put once a week into the beef extract (which had been stopped, however, in March) continued their high rate of division throughout the period of decline of their sister cells, and dicl not show signs of diminished vitality until the first period in June." At this time their rate of division increased 
rapidly. They were put back into the beef extract, but it failed now to have a beneficial effect, and the animals continued to die at a rapid rate. To judge from the appearance of the organisms the new decline was due to a different cause; for, while in the former periods the food vacuoles contained undigested food, at this period the interior was free from food masses. The protoplasm became granular and different from that of a healthy individual. None of the former remedies were now of any avail. "When the last of the $B$-series stock had died in the five hundred and seventieth generation ( $\mathrm{J}$ une I 6 th), it looked as though the cultures were about to come to an end." Extract of the brain and of the pancreas were then tried. To this a favorable response took place at once. The organisms became normal in appearance and began to divide. After forty-eight hours' treatment they were returned to the usual hay infusion. Here they continued to multiply and reached on June 28 th the six hundred and sixty-fifth generation.

There can be no doubt that the periods of clepression that appear in these infusoria kept in cultures can be successfully passed if the animals are introduced into a new environment. Without a change of this sort they will die. Calkins thinks that the effect is produced, not by the new kind of food that is supplied, but by the presence of certain chemical compounds. The beef extract "does not have a direct stimulating effect upon the digestive process and upon division, for, while the organisms are immersed in it, there is a very slow division rate; when transferred again to the hay infusion, however, they divide more rapidly than before."

This brings us back to the idea of the "renewal of youth" through conjugation. Maupas claimed that union of individuals having the same immediate descent is profitless. Calkins suggests that this is due to the similarity in the chemical composition of the protoplasm of the two individuals. When in nature two individuals that have lived under somewhat 
different conditions conjugate, the result should be beneficial, since there takes place the commingling of different protoplasms.

Calkins's work has shown that by means of certain substances much the same effect can be produced as that which is supposed to follow from the conjugation of two unrelated individuals. The presumption, therefore, is in favor of the view that the two results may be brought about in the same way, although we should be careful against a too ready acceptation of this plausible argument; for we have ample evidence to show that many closely similar (if not identical) responses of organisms may be brought about by very different agencies. The experiments seem to indicate that paramoecium might go on indefinitely reproducing by division, provided its environment is changed from time to time. If this is true, it is conceivable that the same thing is accomplished through conjugation. In the light of this possible interpretation much of the mystery connected with the term rejuacnescence is removed, for we see that there is nothing in the process itself except that it brings the organism into a new relation with other substances. Difficult as it assuredly is to understand how this benefits the animal, the experimental fact shows, nerertheless, that such a change is for its good. That there is really nothing in the process of conjugation itself apart from the difference in the constitution of the conjugating individuals is shown by the result that the union of individuals having the same history and kept under the same conditions is of no benefit.

Can we apply this same conception to the process of fertilization in the higher animals and plants? Is the substance of which their bodies are made of such a sort that it cannot go on living indefinitely under the same conditions, but must at times be supplied with a new enviromment? If this could be established, we could see the advantage of sexual reproduction over the non-sexual method. It would 
be extremely rash at present to make a generalization of this kind, for there are many forms known in which the only method of propagation that exists is the non-sexual one. In other words, there are no grounds for the assumption that this is a necessary condition for all kinds of protoplasm, but only for certain kinds.

In the insects, crustaceans, rotifers, and in some plants there are a few species whose egg develops without fertilization. This makes it appear probable that the particular kind of protoplasm of these animals does not absolutely require union from time to time with the protoplasm of another individual having a somewhat different constitution.

There is also an interesting parallel between the effects of solutions on the protozoans in Calkins's experiments and certain results that have been obtained in artificial parthenogenesis. It has been stated, that by brushing the unfertilized eggs of the silkworm moth a larger percentage will develop parthenogenetically; and more recently it has been shown by Matthews that by agitation of the water in which the unfertilized eggs of the starfish have been placed many of them will begin their development. It was first shown by Richard Hertwig that by putting the unfertilized eggs of the sea-urchin in strychnine solutions, they will begin to segment, and I obtained the same results much better by placing the eggs in solutions of magnesium chloride. Loeb then succeeded in carrying the development to a later stage by using a different strength of the same solution, as well as by other solutions. Under the most favorable circumstances some of the eggs may produce larvæ that seem normal in all respects, but whether they can develop into adult sea-urchins has not yet been shown.

These results indicate that one at least of the factors of fertilization is the stimulus given to the egg. On the other hand, the lack of vigor shown by many eggs that have been artificially fertilized indicates that some other result is also 
accomplished by the normal method of fertilization that is here absent. This may mean no more than that as yet we have not found all the conditions necessary to supply the place of the spermatozoon.

In our study of the phenomena of adaptation we have found that sometimes the adaptation is for the benefit of the individual and at other times for the benefit of the species. May it not be true also that the process of sexual reproduction has more to do with a benefit conferred on the race rather than on the individual? In fact, Weismann has elaborated a view based on the conception that the process of sexual reproduction is beneficial to the race rather than to the individual. His idea, however, is not so much that the result is of direct benefit to a particular species, as it is advantageous to the formation of new species from the original one. In a sense this amounts, perhaps, to nearly the same thing, but in another sense the idea involves a somewhat different point of view.

According to his view "the deeper significance of conjugation" and of sexual reproduction is concerned "with the mingling of the hereditary tendencies of two individuals." In this way, through the different combinations that are formed, variations which he supposes are indispensable for the action of natural selection originate. The purpose of the sexual process is solely, according to IVeismann, to supply the variations for natural selection. If it be asked how this process has been acquired for the purpose of supplying natural selection with the material on which it can work, we find the following reply given by Weismann. "But if amphimixis [by which he means the union of sex-cells from different inclividuals] is not absolutely necessary, the rarity of purely parthenogenetic reproduction shows that it must have a widespread and deep significance. Its benefits are not to be sought in the single individual; for organisms can arise by agamic methods, without thereby suffering any loss of vital 
energy; amphimixis must rather be advantageous for the maintenance and modification of species. As soon as we admit that amphimixis confers some such benefits, it is clear that the latter must be augmented, as the method appears more frequently in the course of generations; hence we are led to inquire how nature can best have undertaken to give this amphimixis the widest possible range in the organic world." Nature, Weismann says, could find no more effectual means of bringing about the union of the sexual cells than by rendering them incapable of developing alone. "The male germ-cells, being specially adapted for seeking and entering the ovum, are, as a rule, so ill provided with nutriment that their unaided development into an indiridual would be impossible; but with the ovum it is otherwise, and accordingly the 'reduction division' removes half the germplasm and the power of developing is withdrawn." It can scarcely be claimed, in the light of more recent discoveries, that the reduction division takes place in order to prevent the development of the ovum, for how then could we explain the corresponding division of the male germ-cells?

Whatever means has been employed to bring about the process of sexual reproduction, the guiding principle is supposed by Weismann to be natural selection as stated in the following paragraph: "If we regard amphimixis as an adaptation of the highest importance, the phenomenon can be explained in a simple way. I only assume that amphimixis is of advantage in the phyletic development of life, and furthermore that it is beneficial in maintaining the level of adaptation, which has been once attained, in every single organism; for this is as dependent upon the continuous activity of natural selection as the coming of new species. According to the frequency with which amphimixis recurs in the life of a species, is the efficiency with which the species is maintained; since so much the more easily will it adapt itself to new conditions of life, and thus become modified." 
Thus we reach the somewhat startling conclusion that through natural selection the germ-cells and their protozoan prototypes have been rendered incapable, through natural selection, of reproducing by non-sexual methods, in order that variations may be supplied for the farther action of this same process of natural selection. The speculation has the appearance of arguing in a circle, although if it were worth the attempt an ingenious mind might perhaps succeed in showing that such a thing is not logically inconceivable.

It seems strange that a claim of this sort should have been made, when it is so apparent that the most immediate effect of intercrossing is to swamp all variations that depart from the average. Even if it were true that new combinations of characters would arise through the union of the germ-cells of two different animals, it is certainly true that in the case of fluctuating variations this new combination would be lost by later crossing with average individuals. Moreover, it is well known that variations occur amongst forms that are produced asexually. On the whole, it does not seem to be a satisfactory solution of the problem to assume that sexual reproduction has been acquired in order to supply natural selection with material on which it may work.

Our examination of the suggestions that have been made and of the speculation indulged in, as to what benefit the process of sexual reproduction confers on the animals and plants that make use of this method of propagation, has failed to show convincingly that any advantage to the individual or to the species is the outcome. This may mean, either that there is no advantage, or that we have as yet failed to understand the meaning of the phenomenon. The only light that has been thrown on the question is that a certain amount of renewed vigor is a consequence of this process, but we cannot explain how this takes place. There is also the suggestion that the union of different cells produces the same beneficial effect as a change in the conditions of life produces 
on the organism. The bad effects of close interbreeding that seem sometimes to follow is explicable on this view. This, it seems to me, is the most plausible solution of the question that has been advanced; but, even if this should prove to be the correct view, we need not assume that the process has been acquired on account of this advantage, for there is nothing to show that it has been acquired in this way. 


\section{CHAPTER XIII}

\section{SUMMARY AND GENERAL CONCLUSIONS}

THE question of the origin of the adaptations with which the last three chapters have so largely dealt is one of the most difficult problems in the whole range of biology, and yet it is one whose immense interest has tempted philosophers in the past, and will no doubt continue to excite the imagination of biologists for many years to come. No pretence has been made in the preceling pages to account for the cause of a single useful variation. We have examined the evidence, and from this we believe the assumption justified that such variations do sometimes appear. The more fundamental question as to the origin of these variations has not been taken up, except in those cases in which the adaptive response appeared directly in connection with a known external cause. But these kinds of responses do not appear to have been the source of the other adaptations of the organic world. Our discussion has been largely confined to the problem of the widespread occurrence of adaptation in living things, and to the most probable kinds of known variations that could have given rise to these adaptations. But, to repeat, we have made no attempt to account for the causes or the origin of the different kinds of variation.

Nägeli, in speaking of the methods of the earlier theorists in Germany, remarks with much acumen: "We might have expected that after the period of the Nature-philosophizers, which in Germany crippled the best forces that might have been used for the advance of the science, we should have learnt something from experience, and have carefully guarded 
the field of real scientific work from philosophical speculation. But the outcome has shown that, in general, the philosophical, philological, and æsthetic expression always gets the upper hand, and a fundamental and exact treatment of scientific questions remains limited to a small circle. The public at large always shows a distinct preference for the so-called idealistic, poetic, and speculative modes of expression." The truth of this statement can scarcely be cloubted when in our own time we have seen more than once the same method employed with great public applause. Nowhere is this more apparent than in the writings of many of the followers of Darwin in respect to the adaptations of living things. To imagine that a particular organ is useful to its possessor, and to account for its origin because of the imagined benefit conferred, is the general procedure of the followers of this school. Although protests have from time to time been raised against this unwarrantable way of settling the matter, they have been largely ignored and forgotten. The fallacy of the argument has, for example, been admirably pointed out by Bateson in the following statement: " "In examining cases of variation I have not thought it necessary to speculate on the usefulness or harmfulness of the variations described. For reasons given in Section II such speculation, whether applied to normal structures or to variation, is barren and profitless. If any one is curious on these questions of Adaptation, he may easily thus exercise his imagination. In any case of Variation there are a hundred ways in which it may be beneficial or detrimental. For instance, if the 'hairy' variety of the moor-hen became established on an island, as many strange varieties have been, I do not doubt that ingenious persons would invite us to see how the hairiness fitted the bird in some special way for life in that island in particular. Their contention would be hard to deny, for on this class of speculation the only limitations are those of the ingenuity of the

1 "Materials for the Study of Variation." 
author. While the only test of utility is the success of the organism, even this does not indicate the utility of one part of the economy, but rather the net fitness of the whole." Keeping in mind the admonitions contained in the two preceding quotations, let us pass in review and attempt to analyze more fully the different points that have been considered in the preceding chapters.

It has been pointed out that the evidence in favor of the theory of evolution appears to establish this theory with great probability, although a closer examination shows that we are almost completely in the dark as to how the process has come about. For example, we have not yet been able to determine whether the great groups of animals and plants owe their resemblance to descent from a single original species or from a large number of species. The former view is more plausible, because on it we appear to be furnished with a better explanation of resemblances as due to divergence of character. Yet even here a closer scrutiny of the homologies of comparative anatomy shows that this explanation may be more apparent than real. If discontinuous variation represents the steps by which evolution has taken place, the artificiality of the explanation is apparent, at least to a certain degree.

Admitting that the theory of evolution is the most probable view that we have to account for the facts, we next meet with two further questions, - the origin of species and the meaning of adaptation. These are two separate and distinct questions, and not one and the same as the Darwinian theory claims. The fact that all organisms are more or less adapted to live in some environment appears from ou! examination to have no direct connection with the origin of the adaptation, for, in the first place, it seems probable that, in general, organisms do not respond adaptively to the environment and produce new species in this way; and, in the second place, there is no evidence to show that variation 
from internal causes is so regulated that only adaptive structures arise (although only adaptive ones may survive).

Our general conclusion is then as follows: A species does not arise from another one because it is better adlapted. Selection, in other words, does not account for the origin of ncw species; and adaptation cannot be taken as the measure of a species.

It may sound like a commonplace to state that only those individuals survive and propagate themselves that can find some place in nature where they can exist and leave descendants; and yet this statement may contain all that it is necessary to assume, in order to account for the fact that organisms are, on the whole, adapted. Let us see how this view cliffers from the Darwinian statement of the origination of new forms through a process of natural selection.

According to Darwin's view of the origin of species, each new species is gradually formed out of an older one, because of the advantage that the new individual may have over the parent form. Each step forward is acquired, because it better adapts the organism to the old, or to a new set of conditions. In contrast to this, I have urged that the formation of the new species is, as a rule, quite independent of its adaptive value in regard to the parent species. But after it has appeared, its survival will depend upon whether it can find a place in nature where it can exist and leave descendants. If it should be well adapted to an environment, it will be represented in it by a large number of individuals. If it is poorly adapted, it may only barely succeed in existing, and leave correspondingly fewer descendants. If its adaptiveness falls below a certain point, it can never get a permanent foothold, however often it may appear. Thus the test of survival determines which species can remain in existence, and which cannot, but new species are not manufactured in this way. How far subsequent variations may be supposed to be determined by the survival of certain species and the destruction of others will be discussed presently. 
The difference between the two points of view that we are contrasting can be best brought out by considering the two other kinds of selection which Darwin supposes to have been at work; namely, artificial and sexual selection.

Darwin thinks that the results of artificial selection are brought about by the breeder picking out fluctuating variations. It appears that he has probably overestimated the extent to which this process can be carried; for while there can be no doubt that a certain standard, or fixity of type, can be obtained by selecting fluctuating variations, yet it now seems quite certain that the extent to which this can be carried is very limited. It appears that other factors have also played an important rôle ; amongst these the occasional appearance of discontinuous variation, also the bringing under cultivation of the numerous "smaller species" of De Vries, or the so-called "single variations" of Darwin. Further, the effects of intercrossing in all combinations of the above forms of variations, followed by the selection of certain of the new forms obtained, has been largely employed, and also the direct influence of food and of other external conditions, which may be necessary to keep the race up to a certain standard, have played a part in some cases. The outcome is, therefore, by no means so simple as one might infer from Darwin's treatment of the subject in his "Origin of Species." For these reasons, as well as for others that have been given, it will be evident that the process of artificial selection cannot be expected to give a very clear idea of how natural selection could act.

It is, however, the process of sexual selection that brings out in the strongest contrast the difference between Darwin's main idea of natural selection and the law of the survival of species. In sexual selection the competition is supposed to be always between the individuals of the same species and of the same scx. There can be no doubt in one's mind, after reading "The Descent of Man," that Darwin 
held firmly to the belief that the individual differences, or fluctuating variations, furnish the material for selection. In this way it could never happen that two competing species could exterminate each otfer, because in the one the males were better adorned, or killed each other off on a larger scale, owing to the presence of special weapons of warfare. It is clear that on the law of the survival of species, secondary sexual characters cannot be supposed to have evolved because of their value. Their origin is totally inexplicable on this view. In fact, the presence of the ornaments must be in some cases injurious to the existence of the species. The interpretation of this means, I think, that individual competition cannot be as severe as Darwin believed, and cannot lead to the results that he imagined it does. For this reason it seemed important to make as careful an examination of the claims of the theory of sexual selection as possible, and I hope that the outcome of the examination has shown quite definitely that the theory is incompetent to account for the facts that it claims to explain. It is certain in this case that we are dealing with a phenomenon that must be studied quite apart from any selective value that the secondary sexual organs may have. If this is granted, it will be seen that there is here a wide field for experimental investigation that is practically untouched.

It is evident that the first step that will clear the way to a fuller understanding of the problem of evolution must be a more thorough examination of the question of variation. Darwin himself fully appreciated this fact, yet until within the last fifteen years the study of variation has been largely neglected. With a fuller knowledge of the nature of fluctuating variation as the outcome of the studies of Galton, Pearson, De Vries, and others, and with a fuller knowledge of the possibilities of discontinuous variation as emphasized by Bateson and by De Vries, and, further, with a better knowledge of some of the laws of inheritance in these cases, we 
have begun to get a different conception of how evolution has come about. It may be well, therefore, to go once more over the main points in regard to the different kinds of variation.

While it has been found that no two individuals of a species are exactly alike, yet, taken as a group, the variations appear as though they followed the law of chance. The descendants of the group show the same differences. Thus the group as a whole appears constant, while the individuals fluctuate continually in all directions. This is what we understand by fluctuating variation. If the external conditions are changed, a new "mode" may appear, but the change is generally only a temporary one, and lasts only as long as the new conditions remain. Thus, while the direct influence of the environment may show for a time, the result is transient. Even if it were permanent, there is no evidence that the adaptation of organisms could be accounted for in this way unless the response were useful. It appears that this sometimes really occurs, especially in responses to temperature, to moisture, to the amount of salts in solution, to poisonous substances, etc. In this way, one kind of adaptation is brought about, but there is no evidence that the great number of structural adaptations have thus arisen.

The Lamarckian principle of the inheritance of acquired characters has also been supposed by many writers to be an important source of adaptive variation. An examination of this theory is not found to inspire confidence. We have, therefore, eliminated this hypothesis on the ground that it lacks evidence in its favor, and also because it appears improbable that in this way many of the adaptations in organisms could have been acquired.

Finally, there is the group of discontinuous variations. Of these there may be several kinds, and there is some evidence showing that there are such. For the present we may include all the different sorts under the term mutation, meaning that the new character or group of characters suddenly 
appears, and is inherited in its new form. From the results of De Vries it appears that mutations are sometimes scattering, at least in the case of the evening primrose. From such scattering mutations, the smaller species or varieties (in so far as these do not depend on local conditions) arise. There is here an important point of agreement with Darwin's idea in regard to evolution, inasmuch as he supposed that varieties are incipient species. Our point of view is different, however, in that we do not suppose these varieties (mutations) to have been gradually formed out of fluctuating variations by a process of selection, but to have arisen at once by a single mutation. It also appears that in some cases a single new mutation may develop in a species. We may suppose that the new form might in such a case supplant the parent species by absorbing it, or both may go on living side by side, as will be more likely the case if they are adapted to somewhat different conditions.

A number of writers have supposed that evolution marches steadily forward toward its final goal, which may even lead in some cases to the final but inevitable destruction of the species. By certain writers this view has been called orthogenesis, although at other times the idea is not so much that there is advance in a straight line, as advance in all directions. This appears to be Nägeli's view. It gives a splendid picture of the organic world, as irresistibly marching toward its goal, - a relentless process in some cases, leading to final annibilation, a beneficent process in other cases, leading to the fullest perfection of form of which the type is capable. Compared with the vacillating progress which is supposed to be the outcome of individual selection, this view of progression has a grandeur that appeals directly to the imagination. We must be guided, however, by evidence, rather than by sentiment. The case will, moreover, bear closer scrutiny. If evolution has indeed taken place by the survival of a series of mutations, whose origin has no connection 
with their value, does not this in the end amount to nearly the same thing as maintaining that evolution of organisms has been a steady progress forward, - a progress undirected by external forces, but the outcome of internal development? Admitting that innumerable creations have been lopped off, because they could find no foothold, yet, as Nägeli points out, the result is that, instead of a dense tangle of forms, there has been left relatively few that have been found capable of existing, - those that have found some place in which they can live and leave progeny. From this point of view it may appear, at first thought, that the iclea of erolution through mutations involves a fundamentally different view from that of the Darwinian school of selection; but in so far as selection also depends on the spontaneous appearance of fluctuating variations, the same point of view is to some extent involved, - only the steps are supposed to be smaller. This point is usually ignored and passed over in silence by the Darwinians, but, as Wigand has pointed out, it makes very little difference whether the stages in the process of evolution are imagined to be very small or somewhat larger, so long as they are spontaneous. Selection does not do more than determine the survival of what is offered to it, and does not create anything new.

It is true that if the fluctuating variations that are selected be connected by very slight differences with an almost continuous series of other forms, and if little by little such a series be advanced in a given direction by selection, we get the idea of a continuity, whose advance is determined by selection. It is this conception that appcars to give the theory of natural selection a creative power, which in reality it does not possess, and certainly not in the modified form in which the theory was finally left by Darwin. For Darwin found himself forced to admit that, unless a very considerable number of individuals varied at the same time and in the same direction, the formation of new species could not take place, and 
this idea of many individuals varying at the same time, and in the same direction, at once involves the conception that evolution moves forward by some force resicling in the organism, driving it forwards or backwards. Instability comes, perhaps, nearer to expressing this idea than any other term, and yet to evolve from a protozoan to a man implies the idea of something more than simple unstableness.

The idea that Weismann has touched upon in this connection, namely, that the survival of a given form determines the future course of evolution for that form, is very plausible, and also fits in well with the results of our experience in the field of the inheritance of variations. We see new variations or mutations departing in some or in many characters from the original type, apparently by new combinations or perturbations of those already present. We never expect to see a bird emerge from the egg of an alligator. Thus it appears that by the survival of certain forms the future course of evolution is determined in so far as the new types of mutation are thereby limited. IVeismann means, however, that in this way new plus or minus steps will be indefinitely determined amongst the new fluctuating variations, but this statement is contradicted by our experience of the results of artificial selection. The upper limit does not keep on pushing out indefinitely in the direction determined by the first selection, but is soon brought to a standstill. So that, as far as Weismann's hypothesis is concerned, the idea appears to have no special value. On the other hand, this idea may be fruitful if applied to mutations, but here unfortunately we have not sufficient experience to guide us, and we do not know definitely whether a new character that appears as a mutation will be more likely, in subsequent mutations, to go on increasing in some of the descendants. Thus, while the mutation theory must assume that some new characters will go on heaping up, we lack the experimental evidence to show that this really occurs. It would be also equally important to 
determine, whether, if after several mutations have successively appeared in the same direction, there would be an established tendency to go on in the same direction in some of the future mutations. But here again we must wait until we have more data before we attempt to build up a theory on such a basis.

The attacks on the Darwinian school by the followers of the modern school of experimentalists are with few exceptions based on the assumption that the natural selectionists pretend that their principle is a sort of creative force, - a factor in evolution in the sense of being an active agent. This assumption of the selectionists has led many of them to ignore a fundamental weakness of their theory, namely, the origin of the variations themsclves, although Darwin dicl not overlook or ignore this side of the problem, or fail to realize its importance, as have some of his more ardent, but less critical, followers. They have contented themselves, as a rule, with pointing out that certain structures are useful, and this has seemed to them sufficient proof that the structures must have been acquired because of their value. In contrast to this complacency of the selectionists, we find here and there naturalists who have, from time to time, insisted that the scientific problem of evolution is not to be found in the principle of selection, but in the origin of the variations themselves. It will be clear, from what has been said, that this is our position also, and for us adaptation itself does not appear to be any more a problem that can be examined by scientific methods, than the lack of adaptation. The causes of the change of whatever kind should be our immediate quest. The destruction of the unfit, because they can find no place where they can exist, does not explain the origin of the fit.

Over and beyond the primary question of the origin of the adaptive, or non-adaptive, structure is the fact that we find that the great majority of animals and plants show distinct evidence of being suited or adlapted to live in a special envi- 
ronment, i.c. their structure and their responses are such that they can live and leave descendants behind them. I can see but two ways in which to account for this condition, either (I) teleologically, by assuming that only adaptive variations arise, or (2) by the survival of only those mutations that are sufficiently adapted to get a foothold. Against the former view is to be urged that the evidence shows quite clearly that variations (mutations) arise that are not adaptive. On the latter view the dual nature of the problem that we have to deal with becomes evident, for we assume that, while the origin of the adaptive structures must be due to purely physical principles in the widest sense, yet whether an organism that arises in this way shall persist depends on whether it can find a suitable environment. This latter is in one sense selection, although the word has come to have a different significance, and, therefore, I prefer to use the term surizial of species.

The origin of a new form and its survival after it has appeared have been often confused by the Darwinian school and have given the critics of this school a fair chance for ridiculing the selection theory. The Darwinian school has supposed that it could explain the origin of adaptations on the basis of their usefulness. In this it seems to me they are wrong. Their opponents, on the other hand, have, I believe, gone too far when they state that the present condition of animals and plants can be explained without applying the test of survival, or in a broad sense the principle of selection amongst species.

It will be clear, therefore, in spite of the criticism that I have not hesitated to apply to many of the phases of the selection theory, especially in relation to the selection of the individuals of a species, that I am not unappreciative of the great value of that part of Darwin's idea which claims that the condition of the organic world, as we find it, cannot be accounted for entirely without applying the principle of selection in one 
form or another. This idea will remain, I think, a most important contribution to the theory of evolution. We may sum up our position categorically in the following statements:

Animals and plants are not changed in this or in that part in order to become better adjusted to a given environment, as the Darwinian theory postulates. Species exist that are in some respects very poorly adapted to the environment in which they must live. If competition were as severe as the selection theory assumes, this imperfection would not exist.

In other cases a structure may be more perfect than the requirements of selection demand. We must admit, therefore, that we cannot measure the organic world by the measure of utility alone. If it be granted that selection is not a moulding force in the organic world, we can more easily understand how both less perfection and greater perfection may be present than the demands of survival require.

If we suppose that new mutations and "definitely" inherited variations sudclenly appear, some of which will find an environment to which they are more or less well fitted, we can see how evolution may have gone on without assuming new species have been formed through a process of competition. Nature's supreme test is survival. She makes new forms to bring them to this test through mutation, and does not remodel old forms through a process of individual selection. 


\section{INDEX}

Acclimatization, $3 \mathbf{I} 9$.

Acorn, 24.

Acracids, 160.

Adaptation, definition of, $\mathbf{r}$.

Adjustments, individual, $\mathbf{r}$.

Agassiz, I, 44, 6I.

Agelieus, I73.

Alcohol, I3.

Algre, red, 9.

Alkaloids, 13 .

Allen, 173, 307-310.

Allolobophora, 380 .

Aipheus, 344 .

Ammophila, 5 .

Ammotragus, 208.

Ampelopsis, 403 .

Amphimixis, 448-449.

Amphioxus, 399 .

Ancon race, 3I5-3I6.

Angiostomum, +22 .

Anguillidix, 320 .

Annelids, 19, 20.

Anolis, 10, 194 .

Ant-eater, 227, 228.

Antelope, 6, 206, 208.

Antitoxin, I4.

Ants, I4I-I $46,354,386,407$.

Aphids, 384-386, $419,426$.

Apus, 418.

Archæopteryx, 4I, 42, 53, 54 .

Ardea, 200.

Argus pheasant, 199.

Aristolochia Clematitis, ro, II, I2.

Arsenic, I3.

Artemia, 306.

Ascidians, 417 .

Askenasy, I6I.

Aspalax, 227.

Asterina, 42I-422.

Autenrieth, 58 .

Baboon, 209.

Bacteria, I4, I5, II I, 398.

Baer, Von, 6o, 61, 74, 75.

Bamboo, 3 i3.

Barnacles, 4 I7.

Bartlett, 209, 220.

Bat, 2.

Bates, I83, I86.

Bateson, 273, 278, 453 .
Beard, 210, 2 II, 2 I6.

Beard, J., +35 .

Bee, 2, 3, I9, I 43, I79, 303, 350, 406, 420, $+21,425,43^{8}$.

Beetloven, 218 .

Beetles, 182, 183,189 .

Bell-bird, I98.

Beneden, Van, thI.

Berluura goat, 208.

Biogenetic Law, 7 I.

Birds, 6, 707 ; definition of group, $3^{6}$; evolution of, $4 \mathrm{I}$; instincts of young, 4 ; nest, + ; of paradise, 6 ; teeth of, $3 \circ 1$; variation in, 309-312.

Blind animals, 354.

Blow-fly, $3^{8} 3$.

Bonellia, 353, 4I7.

Born, +24.

Bos, 206.

Boveri, 433.

Bovida, 207.

Branchipus, 306.

Brocadello, +28 .

Brooks, $+\uparrow \mathrm{I}$.

Brown-Séquard, 232, 24r, 250-257.

Buffon, 300.

Bull, 207, 315.

Bütschli, 4.7I.

Butterfly, 3, I84, 389.

Cactus, Io.

Caffein, I3.

California salmon, I9.

Calkins, 443-447.

Callionymus, rgr.

Calocalanus, $\mathbf{1 7 7}$.

Cameron, 425.

Canestrini, 178 .

Canidxe, 308.

Canis, 4 Io.

Carbonnier, I90, I92.

Cardamine, 335.

Cardinalis, 173 .

Cardium, 305.

Cassowary, 202.

Castle, I. $8,321,435,43^{8}$.

Caterpillar, 5, 8, I86.

Cattle, 4 II.

Cats, 209.

Cercopithecus, 208. 
Cervus, 304 .

Chara, 420 .

Charrin, 257.

Chick, 57, 406.

Chrild, 72.

Chinese pheasants, 6 .

Chlorophy\}, 9.

Cicadas, 187, I 88.

Ciona, I.48.

Classification, $3^{1-3^{6}}$.

Classification, scheme of, 38 .

Cockatoo, 6.

Colaptes, 3 Io.

Colias, 185 .

Colonial torms, I27.

Color, I8, I9, 24, I33, 375 .

Coloration, $5,6,7,23,357-360$.

Columba livia, 76 .

Comb of bees, 4 .

Communal marriages, 2 Io.

Competition, IO4, II2, I I9, I 20, I2I, I22, I23.

Composita, $\mathbf{3} 3$.

Cones, 3 Io.

Conklin, 72.

Cope, 49, 259.

Coprida, 183 .

Coral-snakes, I94.

Correlated variation, 94 .

Correlation, I34.

Cottus, I9I.

Crab, 15, 248, 344, 354 .

Crickets, 188 .

Crocodiles, I93.

Crosby, 398.

Cross-fertilization, 21.

Crossing of species, I48, I49, I50.

Crystal, 29.

Cryptocerus, I44.

Ctenophors, 417 .

Cuckoo, I39, I.40, I4I.

Cuénot, 427-428, 435 .

Culicidre, I 88 .

Cunningham, 257-260.

Cuvier, 4. 301 .

Cynocephalus, 209.

Cypridopsis, 392-393.

Cyprinorlonts, igo.

Cypris, 320.

Dall, 260.

1 )allinger, 320 .

Danaids, 160 .

1)ances, I95.

Daplinia, 305, 418 .

Darwin, C., numerous references throughout the text.

Darwin, Erasmus, 223.
Date-palm, 313.

Davenport, 264, 266, 32I.

Dean, 358 .

Death, 370 .

Death, feigning, 4I0, 4II.

Deer, $3 \circ 9$.

Degeneration, I65.

Delamare, 257 .

Descent theory, $3^{I-35}$.

Desmarest, 206.

Desmodium, 403.

Dianthus, I.49.

Didelphys, 4 Io.

Dimorphism, 360 .

Dingoes, $3 \mathrm{I} 4$.

Dinophilus 428 .

Diptera, I80, I88.

Divergence of character, I27, 128.

Dog, 226.

Draba, 288, 289, 290, 292, 294.

Draco, I94.

Dragonet, I9I.

Drill, 209.

Ducks, 94, 314.

Düsing, 423 .

Dutrochet, 320 .

Earthworm, 380, 382, 383, 384, $4^{17}$.

Echidna, 54.

Eciton, 144 .

Egerton, 204.

Egg, 429-430, 432.

Eggs, number of, 19.

Egypt, animals of, 225 .

Egyptian remains of animals, 43,44 .

Eimer, I58, 260.

Eisig, 72.

Electric organs, 22, 132, 372.

Elephant, I IO-I I I, 206, 304.

Emu, 202.

Entoscolax, 353 .

Epilippus, 50.

Equus, 50.

Eristales, 188.

Esmeralua, I82.

Euploids, I6o.

Eustephanus, $20 \mathbf{r}$.

Evolution, 29.

Ewart, 238.

Exercise, I2.

External conditions, I3o.

Eye, I3, I3I, I32.

Eye, evolution of, I3I, I32.

Eye, of flatfish, I37.

Fayal Islands, I2.

Felida, 308. 
Felis, 308 .

Fish, change of color, 16.

Fishes, 7 .

Fishes, secondary sexual character of, I9o.

Flatfish, 137, I38.

Flatworms, 417 .

Fleischmann, 45-57.

Flounders, 228, 346, 347.

Flowers, 9, 17, 21, 342, 399, 422, 429.

Fly, 428 .

Foot of horse, 47.

Forel, 5.

Fossil horses, 52 .

Foxes, 210,4 Io.

Franqueiros cattle, 315 .

Frogs, 193, 320, 382, 405 .

Frogs, cross-fertilization, I5O.

Irruit, down of, 133 .

Fundulus, 16.

Galton, 236, 270-272, 289, $44 \mathrm{I}$.

Gavials, 301 .

Geddes and Thompson, $417,423,426,427$.

Geer, De, I78.

Gegenbaur, 49.

Gelasimus, 177 .

Geoffroy St.-Hilaire, 44, 67, 300-303.

Geological evidence, 39.

Gerbe, 429.

Germinal selection, 154 .

Gibbon, 213.

Gill clefts, 62, 63, 64, 73.

Giraffe, $6,203,229,248-249$.

Glacier, 28.

Glowworm, 23.

Goat, 206, 208.

Gonionema, 399.

Gorilla, 205.

Gothic period, 47, 48.

Gould, 197.

Graba, I24, I25.

Grafting, 153 .

Grasshoppers, 8, I88.

Gray, I26.

Greyhound, I34.

Growth of plants, I7.

Guillemots, I24.

Guinea pigs, 232.

Günther, Igo.

Gymnotus, 132.

Haeckel, 48, 49, 56, 70, 71, 79, 80, 82.

Hartman, 187 .

Heart, 66, 67 .

Heliconids, 160 .

Helix, 344, 345-346.

Hemiptera, I8I.
Heredity, 270.

Hermaphroditic animals, 126.

Hertwig, O., 78, 79, 80, 81, 82, 83 .

Hertwig, R., 447.

Hieracium, 330, 33I.

Hildebrand, $\mathbf{I}_{4} 8$.

Hill, $25^{2}$.

Hipparion, 5I.

Hippeastrum, 148.

His, 7 I, 72 .

Holmes, 72.

Hornbills, 219.

Horns, 229.

Horse, 42, 224.

Horse-chestnut, 24.

Hothura, $f$ Io.

Hottentots, 2 I 2.

Hislson, I40, I95, 409-412.

Humming-birds, 6, 197, 228.

Hurst, $75,76,77,78$.

Huxley, 49, 156, 233.

Hyatt, 259.

Hybrids, I49, 239.

Hydatina, +17.

Hydroides, $34^{8 .}$

Hylobates, 205 .

Hymenoptera, I8I.

Ice, 28.

Ichneumonidæ, I8I.

Idioplasm, 335 .

Immunity, $\mathbf{3}$.

India cattle, 208.

Infanticide, 25.

Inorganic adaptations, 26.

Insectivorous plants, Io.

Insects, coloration of, 7 ; wingless, 228 .

Instinct, 25, I39, I I O, I4I.

Irish elk, 247.

Jackson, 260.

Jaguar, 209.

Japanese cock, 163.

Jennings, 395 .

Jonghe, $3 I_{4}$.

Jordan, 292.

Joseph, 428.

Junco, 3 II.

Kallima, 7, I6I, 162, $35^{8}$.

Kangaroo, 229, 35I.

Katydid, 8.

Kent, W. Saville, I9I.

Kidneys, 66.

Kielmeyer, $5^{8}$.

Kirby, 232.

Kiwi, 354. 
Kölreuter, 149.

Kurschelt, 428.

Labidocera, 393 .

l.amarck, If 6, 222-233.

I amarckian factor, $94,205,211,222,45^{8}$.

Lang, $3+5$.

1 aw of Biogenesis, 30 .

Leaf, resemblance to, 7 .

I teaves, closing of, II.

Leeches, 4 I7.

Leguminosæ, I24.

1. eidy, $4^{6}$.

length of life, 20.

I tenhossek, $\$ 35$.

I,eopard, 209.

Iepidoptera, I72.

I.eptothrix, 320.

Leucophys, 442.

I ichen, 9.

Lillie, 72 .

Limbs of vertebrates, 46 .

Linaria, for.

Linnæan species, 85 .

I innaeus, I9I.

Lion, 6.

Lizards, 7, I6, 17, 193.

I obelia, I48.

I obster, 343 .

Lockwood, 138.

Locusts, I88.

Loeb, 383-392, 447.

Lomaria, 290.

Lowell lectures, $6 \mathrm{I}$.

Lumbriculus, I5.

I.uminous organs, 133 .

1.ymnxa, 305, 322 .

Lythrum, 365-370.

Machines, 26, 27, 28.

Mclntosl, I76.

McNeill, 204.

Macropus, I92.

Malva, for.

Nammalia, origin, 54, 202.

Man, 2 ro.

Miarsh, 49.

Matthews, 447.

Nituchamp, 315 .

Malupas, $4+1,4+2,445$.

May-flies, I9, 353, $3^{89}$.

Meiul, 72.

Neckel, 59,60 .

Nelinism, 209 .

Melospiza, 3 Ir.

Mentel, $27^{8}-286,433,436$.

M.soliiplus, $5 \mathrm{I}$.

Mimosa, fo4.
Minnow, 16.

Minot, 433.

Mirabilis, I49, I5o.

Mivart, I36, I37.

Mole, I, 2, I8, 227.

Mole-cricket, I, 2.

Molothrus, 140.

Monkeys, 207, 208.

Mons, Van, 332.

Montgomery, $43^{2}$.

Noor-hen, 453.

Moquin-Tandon, 303.

Morton, Lord, 238.

Moschus, 206.

Moths, $184,3^{87}, 3^{88}$.

Moussu, 257.

Mozart, 218.

Mulberry, $3 \mathbf{I} 3$.

Müller, I82, 188 .

Müller, Fritz, I 48 .

Muscles, 12.

Mycetes, 205.

Myzostomum, 422 .

Nägeli, I6I, 325-339, 459 .

Natural selection, I04-I07, I08, I09, II0, etc.; definition of, II7.

Nauplius, 69.

Nectar, I24.

Nectar-feeding insects, 126, I27.

Nectarines, 134 .

Negroes, 2 I 2.

Nematode, number of eggs, I Io.

Nematus, 425 .

Nemertian worms, 176.

Neo-Lamarckians, 240, 259-260.

Nepenthes, Io.

Nephela, 78 .

Nest of birds, $4,407-408$.

Neuters, I+2.

Nicotine, I3.

Nostocs, 320.

Notochord, 64, 65 .

Nussbaum, 424 .

Ocneria, 428 .

Enothera, 294-297.

Oken, 56, $5^{8}$.

Old agre, 2I, 25 .

Onites, 232.

Onychodromus, 442.

Opossum, fro.

Organs of little use, 22.

" Origin of Species," 129.

Ornithorynchus, 54.

Orobanchia, 330.

Osborn, 259. 
Oscillaria, 320.

Ostrich, 203, 354.

Uxalis, 290, 404 .

Oxen, $30+$.

Uxide, 29.

Packard, 23I, 260.

Paludina, 320, 322.

Pangenesis, 233-2.40.

Papilio, 158, 360, 388 ; polyxenes, 3 .

Paradisea, I97.

Paramacium, 395-398, 442-447.

Parusitism, 352-353.

Parker, 393.

Parrots, 6.

Partridge, 4Io.

Passerella, 3 II.

Passiflora, I48.

Pavo, 3I7.

Peach, I34.

Peacock, 200, 3I7-3I8.

Peafowl, I98.

I'carson, 265, 267, 268-270.

Peas, 28I-280.

Peckliam, I78, 408.

Pelohates, 42 I.

Pfliger, 424, 430 .

thosphorescent organs, 22, I33.

Plyysa, 320, 322.

Pigeons, selection in, Io2.

Pipilo, 3 I I.

Pisum, 278.

Pithecia, 208.

Ilanaria, 380.

Planarians, 394.

Piants, 403, 4I5; color of, 24 ; influence of light, 17 .

Plato, 304.

Plover, 202.

Poisons, I3, I4, I5, 18, 20, 377 .

Polar bear, 6.

Pollen, 2, 125.

Polygon, 262.

Porthesia, 389.

Primula, $36 \mathrm{I}-365$.

Prionide, I82.

Probosces of insects, 127.

Protective coloration, 5, 6, 16, 158, I59.

I'roteus, 227.

Protohippus, 5 I.

Przibram, 347.

Psyche, 419.

Ptarmigan, 5.

Pyrodes, 182.

Quetelet, 289.

Quiscalus, major, r73.
Rablyit, Porto Santo, 316-3I7.

Rabbits, 304.

Riabbits in Australia, II2.

Race-horse, I34.

Ramunculus, 305.

Kay-florets, I35.

Rays, electric organs of, 22.

Réanmur, 388.

Recapitulation theory, 58-83.

Reduction division, $432-433$.

Regeneration, 15, 16, 27, 379.

Regulations, 27, 28.

Reproductive organs, I9.

Reptiles, fossil, 52, 53 .

Reugger, 205.

Rhododendron, 330.

Rhynchxa, 2or.

Riley, 424.

Rivers, 28.

Robinia, 40.4.

Romanes, I32, 250-256, 4I2.

Rose, 307 .

Rothert, 398.

Rotiters, I I8, 353, 424 .

Roulin, 304 .

Roundworms, I76, 353 .

Rudimentary organs, 22.

Ryder, 260.

Sacculina, 353 .

Sixclis, Io.

Salmon, I9.

Sialter, $3 \mathbf{I} 4$.

Salvin, 2or.

saphirina, $\mathbf{I} 76$.

sivages, 2 Io.

Saw-flies, $\$ 25$.

scarlet tanager, 198.

Schaefer, 244.

Sclater, I98.

Sicops, 312.

Sicott, I $48,259$.

Siea-anem one, 34 I.

Siea-urchin, $3+1$.

Secondary sexual characters, 2 I.

Selection, I 16.

Selection, artificial, 9 I, 92, 96, 97, 98.

Self-fertilization, I 26.

Semper, 260.

Setchel, 320.

Sexual characters, secondary, 372-374.

Sexual selection, $\mathbf{6} 67$.

Sliarp, 350, 425.

Sheep, 208.

Sherrington, 244.

Shrew mice, 206.

Silkworm, 428,447 . 
Silver-bill, 4 IO.

Sirex, I8I.

Siricide, I8I.

Sitaria, 194.

skin, thickening of, 12, 13.

Skull, 37, 65.

Skunk, 3 .

Slaves of ants, I+I.

Sleep in plants, 404 . .

Sloth, 229.

Snail, 417 .

Snails, color of, 23.

Snakes, I4, I93-194, 227.

Snowy owl, 6.

Solenobia, 4 I9.

Soles, 137, 228.

Sparassus, 178 .

Sparrow, 200; English, II2.

Species, 31, 32, 33; adaptation for good of, I9: sharp separation of, I3I.

Spencer, 240-246, 247, 290.

Spermatozoa, I50, 430-433.

Splinx, 186, 388.

Spiders, 177-178, 179, to6; web, 3.

Spirogyra, 420 .

spontaneous variability, I34.

Spores, 322.

squilla, 177 .

Siquirrels, 2 IO.

stag-beetle, 179.

Stags, 203-204, 219.

Sterility, I 77-I52.

Strasburger, 395 .

Stridulating organs, $188,189$.

Struggle for existence, 109, IIo.

Stylonvchia, $4+2$.

Survival of the fittest, 107, I08, 109, II7.

Sutton, 432.

Swallow, II5.

Sweating, 12.

Tadpole, 32I, +28 .

'Tail, 2.

'lanager, 6 .

'Tapeworm, 353; number of eggs, IIo.

'Taraxacum, 305.

Tear-sacs, 206.

Teeth, bircl's, 67, 68.

'lelegonv, 95, 234, 237, 238, 239.

'Tenthredinida, I8 I, +25 .

Termite, number of eggs, I Io.

Termiticler, 350.

'Thrush, II 5 .

'Tipulax', 188.

'load, 7.

lorpecto, 132.

Towle, 392 .
Transitional forms, 42.

Transmutation theory, $3 \mathbf{I}, 34$.

Traquair, 138.

Treadwell, 72.

Treat, 42,4 .

Tree-frogs, 7 .

Trichina, 353 .

Trifolium, to4.

Triton, 193 .

Turkeys, $3^{1} 4$.

Turnix, 20I, 202.

Turtles, I93.

Umbelliferæe, $\mathbf{1 3 5}$.

Uria lacrymans, I24.

Utricularia, ro.

Vanessa, 360.

Variability, 92, 93, 95, 96, 318-3I9.

Variation, 26I, 340.

Variation, fluctuating, 100, I 18, I23.

Variation under domestication, 136 .

Varieties, 106, 107, I.48.

Varigny, De, 303-306, 314-315, 322.

Venus fly-trap, 9.

Verbascum, I48, I49.

Vertebrates, evolution of, 40,45 .

Vilmorin, 303, 3I4.

Vinson, 178 .

V'ries, De, 97, 278, 289-298, 340.

Vulpine, 209.

Wallace, 7, I62, I86, 202, 221, 249.

Walrus, 203.

Walsh, I8I.

Walther, 59 .

Wasp, 3, 5, 408, 409.

Waterton, 198 .

W'el, spider's, 3,4 .

Weir, I7I.

Weismann, I5t-I66, 44I, 448-450.

Westwood, 188.

Whale, 227, 30I.

Wilson, E. B., 72.

Wing of bat, 2 .

IVolf, 308,376 .

Wolves, 412.

Women, 210.

Woodpecker, 228.

Wounds, healing of, 15 .

Yarrell, 138.

Yung, $42+,+36$.

Zcluu cattle, 208.

Zeleny, 348 .

Zoea, 69,70 . 


\title{
REGENERATION
}

\section{By THOMAS HUNT MORGAN, Ph.D.}

\author{
Professor of Biology" an Bryn Maur College; author of "The Development \\ of the Frog's Egrg," etc.
}

\section{Cloth 8vo \$3.00 net}

"This volume is the outcome of five lectures on "Regeneration and Experimental Embryology," given in Columbia University, in January, 1900. The subjects dealt with in the lectures are here more fully treated and are supplemented by the discussion of a number of related topics. During the last few years the problems connected with the regeneration of organisms have interested a large number of biologists, and much new work has been done in this field, especially in comection with the regenerative phenomena of the egg and early embryo. The development of isolated cells or blastomeres has, for instance, aroused widespread interest. It has become clearer, as new discoveries have been made, that the latter phenomena are only special cases of the general phenomena of regeneration in organisms, so that the results have been treated from this point of view in the present volume, which, however, has a wider bearing than simply as a treatment of the problems of regeneration." - From the Author's Preface.

"The presentation is thorough and comprehensive; besides original researches of the author, it involves a great deal of recapitulation and verification of experiments performed by others, with analyses and criticisms of the conclusions drawn from the phenomena or of theories advanced in explanation. Dr. Morgan is fortunate in possessing experience, depth of insight, and a judicial habit of mind that give hin special fitness for his task." - The Nation.

"It is rare indeed to find a book which contains so large an amount of research work and which is at the same time of such general interest and importance. The book will undoubtedly take a prominent place among the standard biological works of the world." - E. G. C. in Science.

\section{THE MACMILLAN COMPANY}

66 FIFTH AVENUE, NEW YORK 


\title{
THE DEVELOPMENT OF THE FROG'S EGG
}

\author{
An Introduction to Experimental Embryology
}

\section{By THOMAS HUNT MORGAN, Ph.D.}

Professor of Biology' in Bry'n Mawr College

\section{Cloth 8vo \$1.6o net}

"Professor Morgan's book gives us a much-needed text-book for both student and instructor, and it should stimulate and greatly aid investigation by pointing out the wide field the frog's egg still offers to embryological research."

- American Journal of Science.

"A clear, succinct, and comprehensive account of all the known phases of the fertilization and development of the frog's egg. ... That the statements are clear and intelligible as possible, the reader may feel sure.... The medical student should master it. .. The general student or the reader who is interested in the matter of the physical basis of heredity will find here the fundamental facts regarding the first beginnings of life and the structure of the egg as well as the sperm cell."

- The Indepindent.

\section{THE MACMILLAN COMPANY}

66 FIFTH AVENUE. NEW YORK 




10989 


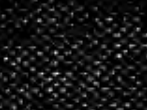

$\alpha+\alpha$

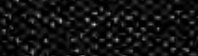

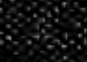

$8 \%$

$8+8 \%$

20\%

$+28 \%$

$+8$

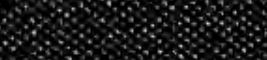

W

No

N

(

N

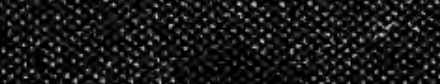

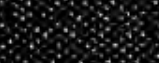

$+2$

$+3$

30

20

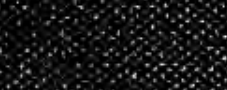

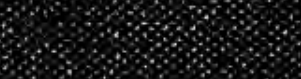

40

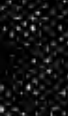

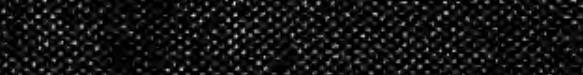

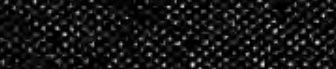

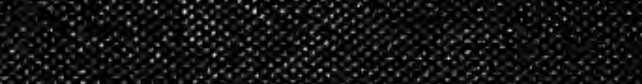

$\because 2$

$+\%$

$4 \%$

$$
\text { (3) }
$$

